\title{
Asymmetric Total Synthesis of Clionastatins A and B
}

\author{
Wei Ju, ${ }^{\dagger}$ Xudong Wang, ${ }^{\dagger}$ Hailong Tian and Jinghan Gui* \\ CAS Key Laboratory of Synthetic Chemistry of Natural Substances, Center for Excellence in Molecular \\ Synthesis, Shanghai Institute of Organic Chemistry, University of Chinese Academy of Sciences, Chinese \\ Academy of Sciences, 345 Lingling Road, Shanghai 200032, China \\ ${ }^{\dagger}$ These authors contributed equally to this work. *Correspondence to: guijh@sioc.ac.cn.
}

\section{Supporting Information}

\section{Table of Contents}

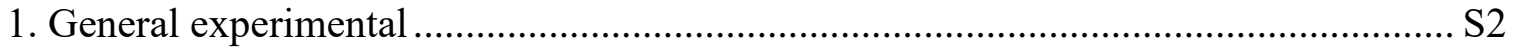

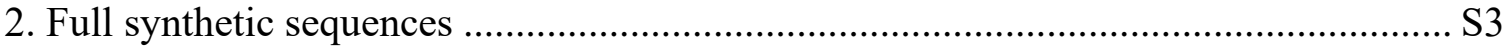

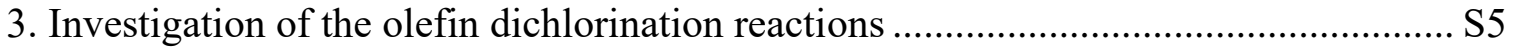

4. Stereochemical rationale for the olefin dichlorination reactions .............................. S6

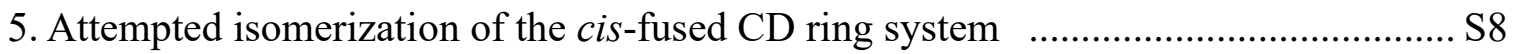

6. Experimental procedures and characterization data for compounds $7-29 \ldots \ldots \ldots \ldots \ldots . . . .59$

7. NMR comparison of synthetic and natural clionastatins A and B .......................... S32

8. Discussion of some discrepancies in the ${ }^{13} \mathrm{C}$ NMR data......................................... S39

9. Structural revision of clionastatins................................................................... S42

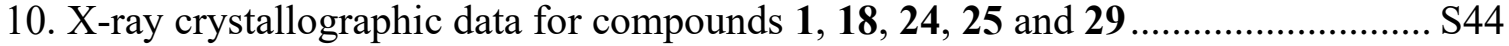

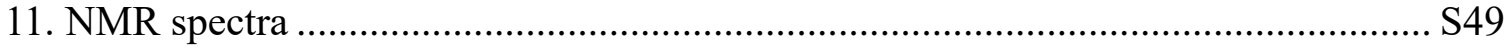

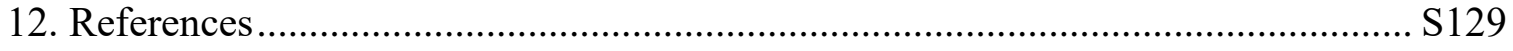




\section{General experimental}

All reactions utilizing air- or moisture-sensitive reagents were carried out in flame-dried glassware under an argon atmosphere, unless otherwise stated. Dry tetrahydrofuran (THF), dichloromethane (DCM), toluene (PhMe), N,N-dimethylformamide (DMF) and acetonitrile $\left(\mathrm{CH}_{3} \mathrm{CN}\right)$ were obtained by passing the HPLC grade or pre-dried solvents through activated alumina columns. Triethylamine $\left(\mathrm{Et}_{3} \mathrm{~N}\right)$, thionyl chloride $\left(\mathrm{SOCl}_{2}\right)$, carbon tetrachloride $\left(\mathrm{CCl}_{4}\right)$ and dioxane were distilled from $\mathrm{CaH}_{2} . \mathrm{N}$-methylmorpholine (NMM) was distilled from $\mathrm{BaO}$. Reagents were purchased at the highest commercial quality and used without further purification, unless otherwise stated. Reactions were magnetically stirred and monitored by thin layer chromatography (TLC) with $0.15-0.2 \mathrm{~mm}$ pre-coated silica gel $(10-40 \mu \mathrm{m})$ plates, using UV light as the visualizing agent or ethanolic phosphomolybdic acid and heating as developing agents. All the chlorinated compounds were visualized by Seebach's "magic" stain. Flash chromatography was performed with silica gel (200-300 mesh) under pressure. Yields refer to chromatographically and spectroscopically ( ${ }^{1} \mathrm{H}$ NMR) homogeneous material, unless otherwise stated. NMR spectra were recorded on Bruker-400, Bruker-500 and Agilent-600 spectrometer. ${ }^{1} \mathrm{H}$ NMR spectra were calibrated using residual undeuterated solvent as an internal reference $\left(\mathrm{CDCl}_{3}: 7.26\right.$ ppm; $\mathrm{CD}_{3} \mathrm{OD}: 3.31 \mathrm{ppm}$ ) and ${ }^{13} \mathrm{C}$ NMR spectra were calibrated against the deuterated solvent peak $\left(\mathrm{CDCl}_{3}\right.$ : $\left.77.2 \mathrm{ppm} ; \mathrm{CD}_{3} \mathrm{OD}: 49.0 \mathrm{ppm}\right)$. The following abbreviations were used to explain multiplicities: $\mathrm{s}=$ singlet, $\mathrm{d}=$ doublet, $\mathrm{t}=$ triplet, $\mathrm{q}=$ quartet, $\mathrm{m}=$ multiplet, br $=$ broad. IR spectra were collected on Avatar 330 FT-IR spectrometer. Melting points were determined on SGW X-4 microscopic melting point apparatus and were uncorrected. Optical rotations were determined on JASCO P-1030 Polarimeter in the solvent indicated. High-resolution mass spectra were recorded on IonSpec 4.7 Tesla FTMS or Bruker Daltonics, Inc. APEXIII 7.0 TESLA FTMS. 


\section{Full synthetic sequences}

\section{Scheme 1. Asymmetric Total Synthesis of Clionastatin A and B}
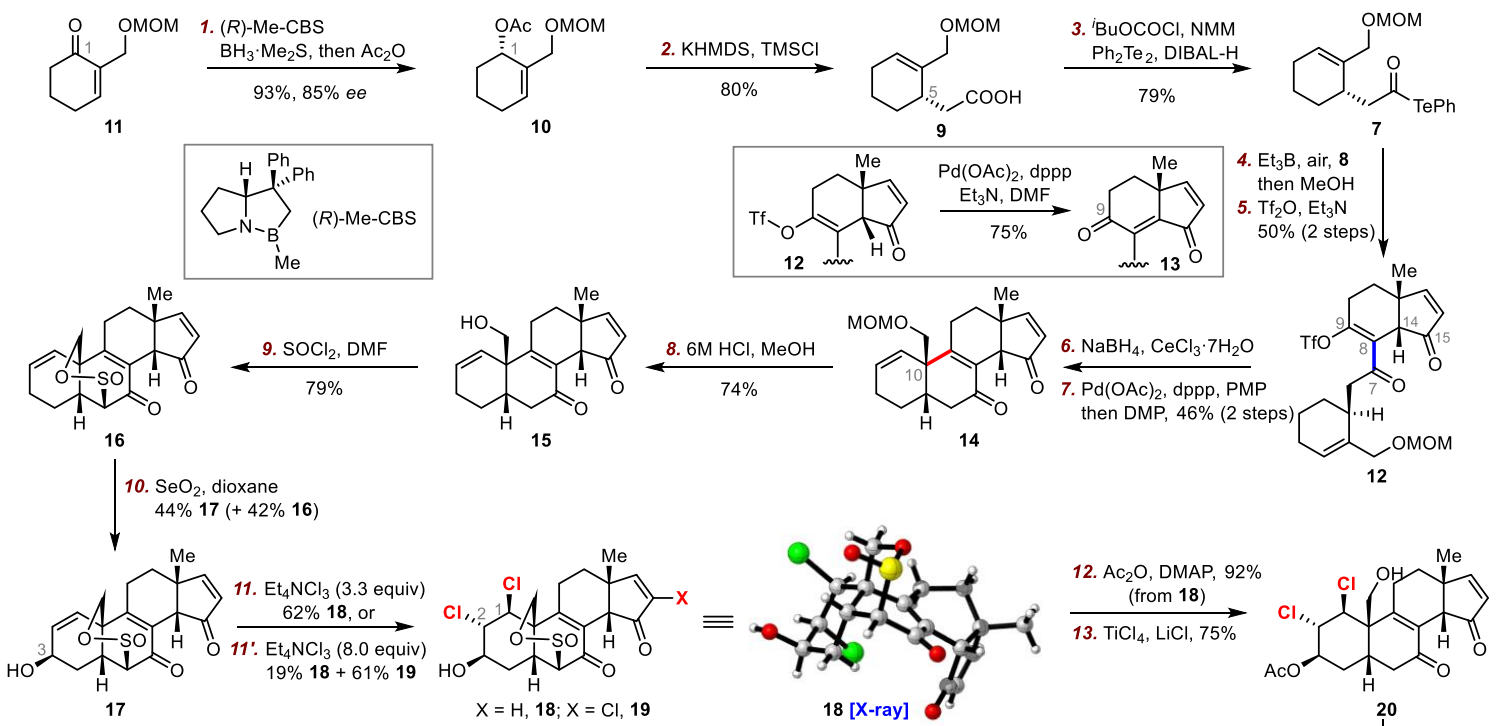

14. $\mathrm{PPh}_{3}, \mathrm{CCl}_{4}, 63 \%$

15. $m$-IBX, $\mathrm{Ph}_{2} \mathrm{Se}_{2}$
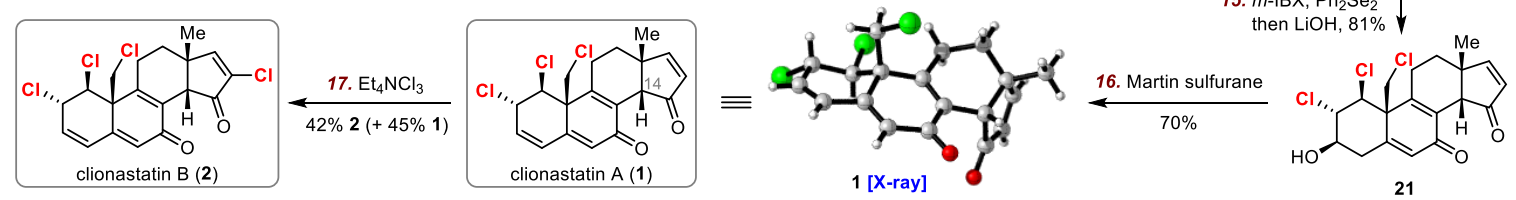

Reagents and conditions: (1) (R)-CBS ( 0.2 equiv), $\mathrm{BH}_{3} \cdot \mathrm{Me}_{2} \mathrm{~S}$, ( 1.5 equiv), THF, -78 to $0{ }^{\circ} \mathrm{C}, 10 \mathrm{~min}$; then $\mathrm{Ac}_{2} \mathrm{O}$ (1.5 equiv), DMAP (0.1 equiv), $\mathrm{Et}_{3} \mathrm{~N}$ (1.5 equiv), DCM, rt, $30 \mathrm{~min}, 93 \%, 85 \%$ ee; (2) KHMDS (2.0 equiv), TMSCl (2.5 equiv), toluene, $\mathrm{rt}$ to $80^{\circ} \mathrm{C}, 4 \mathrm{~h}, 80 \%$; (3) $i$-BuOCOCl (1.2 equiv), $\mathrm{Ph}_{2} \mathrm{Te}_{2}$ (0.65 equiv), NMM (1.2 equiv), DIBAL-H (1.4 equiv), THF, $-20^{\circ} \mathrm{C}, 30 \mathrm{~min}, 79 \%$; (4) $\mathrm{Et}_{3} \mathrm{~B}(3.0$ equiv), 8 (1.0 equiv), DCM, $-40{ }^{\circ} \mathrm{C}, 30 \mathrm{~min}$; then $\mathrm{MeOH}, 12 \mathrm{~h}$; (5) $\mathrm{Et}_{3} \mathrm{~N}$ (3.0 equiv), $\mathrm{Tf}_{2} \mathrm{O}$ (2.0 equiv), $\mathrm{DCM},-78{ }^{\circ} \mathrm{C}, 40 \mathrm{~min}, 50 \%$ (2 steps); (6) $\mathrm{NaBH}_{4}$ (3.0 equiv), $\mathrm{CeCl}_{3} \cdot 7 \mathrm{H}_{2} \mathrm{O}$ (3.0 equiv), $\mathrm{MeOH}, 0{ }^{\circ} \mathrm{C}, 30$ min; (7) $\mathrm{Pd}(\mathrm{OAc})_{2}$ ( 0.2 equiv), dppp (0.2 equiv), PMP (2.0 equiv), $\mathrm{CH}_{3} \mathrm{CN}, 80^{\circ} \mathrm{C}, 5 \mathrm{~h}$; then DMP (5.0 equiv), DCM, 30 min, 46\% (2 steps); (8) $6 \mathrm{M} \mathrm{HCl}$ (10.0 equiv), $\mathrm{MeOH}, 50{ }^{\circ} \mathrm{C}, 10 \mathrm{~h}, 74 \%$; (9) $\mathrm{SOCl}_{2}$ (3.0 equiv), DMF (10.0 equiv), DCM, rt, $3.5 \mathrm{~h}, 79 \%$; (10) $\mathrm{SeO}_{2}$ (2.0 equiv), $\mathrm{NaH}_{2} \mathrm{PO}_{4}$ (1.0 equiv), quartz sand, dioxane, $140{ }^{\circ} \mathrm{C}, 2 \mathrm{~h}, 44 \% 17$ ( $+42 \%$ 16); (11) $\mathrm{Et}_{4} \mathrm{NCl}_{3}$ (3.3 equiv), DCM, $-78^{\circ} \mathrm{C}$ to rt, $10 \mathrm{~min}$, $62 \% 18 ;\left(11^{\prime}\right) \mathrm{Et}_{4} \mathrm{NCl}_{3}$ (8.0 equiv), DCM, rt, 30 min, $61 \% 19$ ( + 19\% 18); (12) $\mathrm{Ac}_{2} \mathrm{O}$ (2.0 equiv), DMAP (0.2 equiv), $\mathrm{Et}_{3} \mathrm{~N}$ (3.0 equiv), DCM, rt, $30 \mathrm{~min}, 92 \%$; (13) $\mathrm{TiCl}_{4}$ (5.0 equiv), $\mathrm{LiCl}$ (5.0 equiv), DMF, 90 ${ }^{\circ} \mathrm{C}, 4$ h, 75\%; (14) $\mathrm{PPh}_{3}$ (5.0 equiv), $\mathrm{CCl}_{4}, 100{ }^{\circ} \mathrm{C}$ (MW), 4 h, 63\%; (15) $m$-IBX (1.5 equiv), $\mathrm{Ph}_{2} \mathrm{Se}_{2}(0.5$ equiv), toluene, $110^{\circ} \mathrm{C}, 2 \mathrm{~h}$; then $\mathrm{LiOH} \cdot \mathrm{H}_{2} \mathrm{O}$ (5.0 equiv), THF/ $\mathrm{H}_{2} \mathrm{O}(1: 1 \mathrm{v} / \mathrm{v}), \mathrm{rt}, 10 \mathrm{~min}, 81 \%$; (16) Martin sulfurane (3.0 equiv), DCM, rt, 1 h, 70\%; (17) $\mathrm{Et}_{4} \mathrm{NCl}_{3}$ (8.0 equiv), DCM, rt, 30 min, $42 \% 2$ ( + $45 \%$ 1). Abbreviations: MOM, methoxymethyl; Ac, acetyl; DMAP, $N, N$-4-dimethylaminopyridine; rt, room temperature; KHMDS, potassium bis(trimethylsilyl)amide; TMS, trimethylsilyl; NMM, $N$ methylmorpholine; DIBAL-H, diisobutylaluminum hydride; Tf, trifluoromethanesulfonyl; dppp, 1,3- 
bis(diphenylphosphino)propane; PMP, 1,2,2,6,6-pentamethylpiperdine; DMP, Dess-Martin periodinane; DMF, $N, N$-dimethylformamide; MW, microwave; $m$-IBX, meta-iodoxybenzoic acid; Martin sulfurane, $\mathrm{Ph}_{2} \mathrm{~S}\left(\mathrm{OC}\left(\mathrm{CF}_{3}\right)_{2} \mathrm{Ph}\right)_{2}$.

Scheme 3. Investigation of the olefin dichlorination reaction.

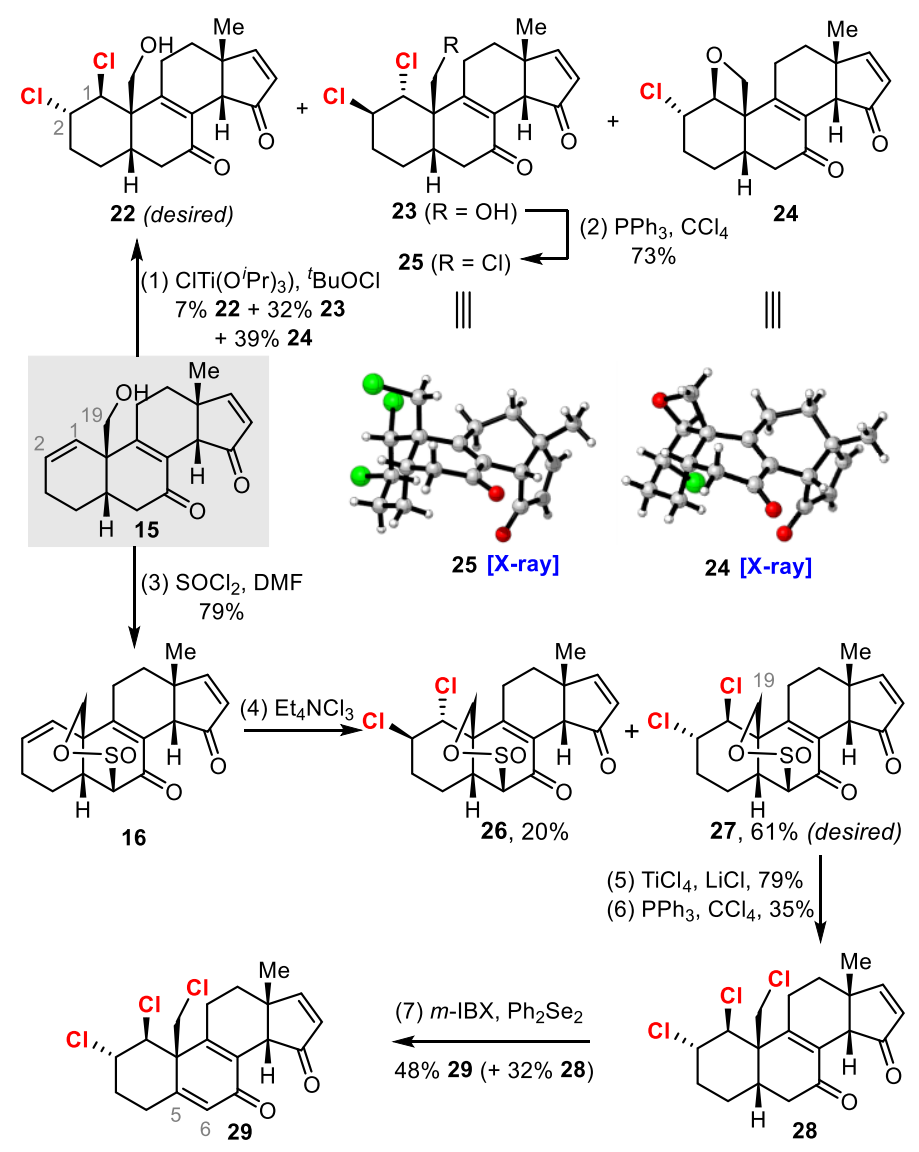

Reagents and conditions: (1) $\mathrm{ClTi}\left(\mathrm{O}^{i} \mathrm{Pr}\right)_{3}\left(1.5\right.$ equiv), ${ }^{t} \mathrm{BuOCl}$ (1.5 equiv), $\mathrm{DCM}, \mathrm{rt}, 30 \mathrm{~min}, 7 \% 22+$ $32 \% 23+39 \%$ 24; (2) $\mathrm{PPh}_{3}$ (3.0 equiv), $\mathrm{CCl}_{4}, 100{ }^{\circ} \mathrm{C}$ (MW), $2.5 \mathrm{~h}, 73 \%$; (3) $\mathrm{SOCl}_{2}$ (3.0 equiv), $\mathrm{DMF}$ (10.0 equiv), DCM, rt, 3.5 h, 79\%; (4) $\mathrm{Et}_{4} \mathrm{NCl}_{3}$ (3.0 equiv), DCM, rt, 30 min, 20\% 26, 61\% 27; (5) $\mathrm{TiCl}_{4}$ (5.0 equiv), $\mathrm{LiCl}$ (5.0 equiv), DMF, $90{ }^{\circ} \mathrm{C}, 2 \mathrm{~h}, 79 \%$; (6) $\mathrm{PPh}_{3}$ (3.0 equiv), $\mathrm{CCl}_{4}, 100{ }^{\circ} \mathrm{C}(\mathrm{MW}), 4$ h, 35\%; (7) $m$-IBX (1.5 equiv), $\mathrm{Ph}_{2} \mathrm{Se}_{2}$ ( 0.2 equiv), toluene, $110{ }^{\circ} \mathrm{C}, 6.5 \mathrm{~h}, 48 \% 29(+32 \% 28$ ). 


\section{Investigation of the olefin dichlorination reactions}

As shown in Table S1, substrate-controlled olefin dichlorination of homoallylic alcohol 15 with $\mathrm{Et}_{4} \mathrm{NCl}_{3}$ (Mioskowski reagent) ${ }^{1}$ afforded undesired dichloride 23 as the major product, along with desired dichloride 22 and undesired oxetane 24 as minor products (entry 1). Treatment of 15 with $\mathrm{ClTi}\left(\mathrm{O}^{i} \mathrm{Pr}\right)_{3}$ and ${ }^{t} \mathrm{BuOCl}$ (Burns's condition without the chiral ligand) $)^{2-5}$ resulted in the preferential formation of undesired $\mathbf{2 3}$ and $\mathbf{2 4}$ (entry 2). Although the use of chiral ligands increased the ratio of 22 to 23 (entries 3, 4), these conditions led to low conversion of $\mathbf{1 5}$ and undesired formation of oxetane $\mathbf{2 4}$. Moreover, adding $\mathrm{Ti}\left(\mathrm{O}^{i} \mathrm{Pr}\right)_{4}$ as an additive or using $\mathrm{Cl}_{2} \mathrm{Ti}\left(\mathrm{O}^{i} \mathrm{Pr}\right)_{2}$ as the Lewis acid ${ }^{6}$ didn't improve the product distribution (entries 5, 6), furnishing desired 22 in small amount. Interestingly, further screening of various Lewis acids revealed that the use of $\mathrm{SnCl}_{4}{ }^{7}$ could suppress the formation of oxetane 24 (entries 7-10). However, it gave rise to undesired diastereomer 23 in $60 \%$ isolated yield, and desired diastereomer 22 only in trace amount.

Table S1. Investigation of the $\mathbf{C 1 9}$ hydroxyl group-directed olefin dichlorination reaction

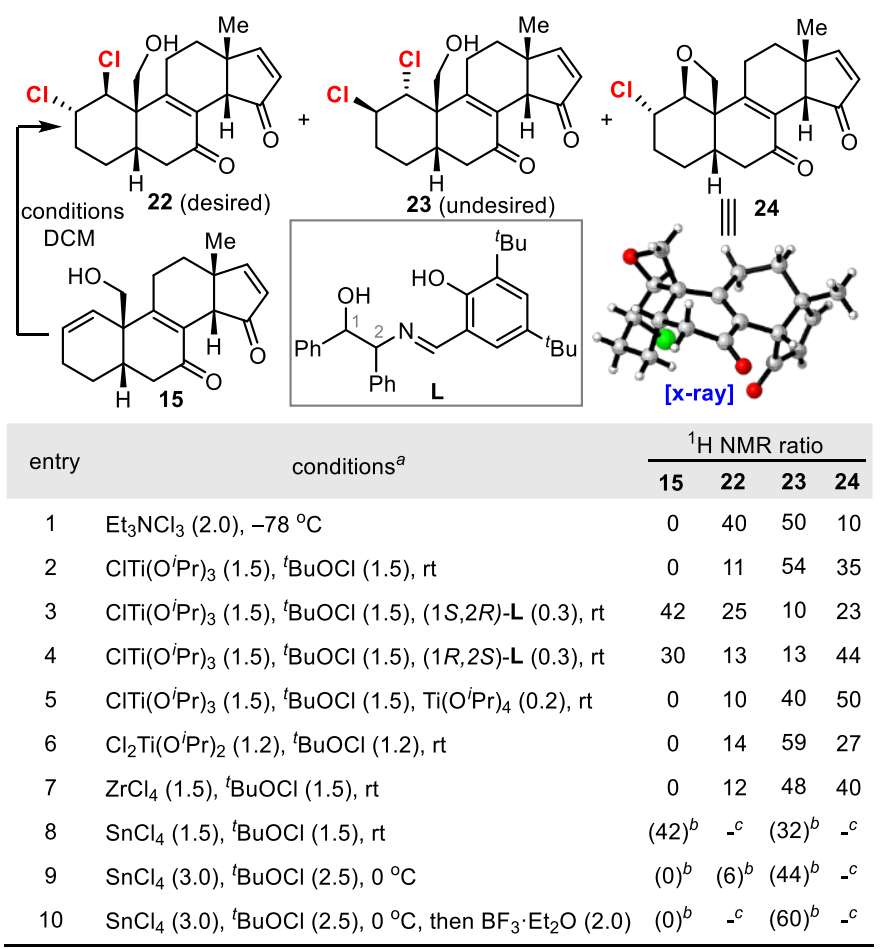

${ }^{a}$ Equivalents of the reagents were shown in parentheses. ${ }^{b}$ Isolated yields were shown in parentheses. 'Isolated yield $<5 \%$. 


\section{Stereochemical rationale for the olefin dichlorination reactions}

As shown in Scheme S1, the stereochemical outcome for the olefin dichlorination of $\mathbf{1 5}$ and 16 can be interpreted by the Fürst-Plattner rule (the trans-diaxial effect), which states that a cyclohexene derivative (such as a chloronium ion) is attacked to obtain the diaxial product and mechanistically to avoid a twist boat conformation [we gratefully thank one of the anonymous reviewers for this suggestion]. Due to the conformational flexibility of 15, this compound may exist as two possible conformations, i.e. the pseudo axial conformation (A) and the pseudo equatorial conformation (B) (Scheme S1A). The chloronium ion $\mathbf{S} 1$ with the pseudo axial conformation for $\mathrm{C} 19$ was attacked by the chloride to afford diaxial dichloride $\mathbf{S 2}$ according to the Fürst-Plattner rule. Further equilibration of S2 by ring-flip then led to the lower energy conformer 23. Alternatively, the chloronium ion $\mathbf{S 3}$ with the pseudo equatorial conformation for $\mathrm{C} 19$ could be attacked either by the chloride intermolecularly to afford diaxial dichloride 22, or by the C19 hydroxyl group intramolecularly to give oxetane 24. We surmised that 15 without an element of conformational lock might exist as a nearly 1:1 ratio of two possible conformations (A/B), which led to the formation of $\mathbf{2 3}$ equal to the total amount of 22 and $\mathbf{2 4}$ (entries 1, 2, Table $\mathrm{S} 1)$. However, due to the presence of the sultine moiety, 16 was likely to exist as the pseudo equatorial conformation preferentially (Scheme S1B). The attack of the chloride to the resulting chloronium ion $\mathbf{S 4}$ led to the formation of $\mathbf{2 7}$ as the major product (path a). The alternative attack of the chloride via path $\mathrm{b}$ to afford $\mathbf{S 5}$ with a twist boat conformation was disfavored, which gave rise to $\mathbf{2 6}$ as the minor product. 


\section{Scheme S1. Stereochemical rationale for the olefin dichlorination of 15 and 16}

A. Possible pathways for dichlorination of 15 to afford 22, 23 and 24 (without conformational lock):

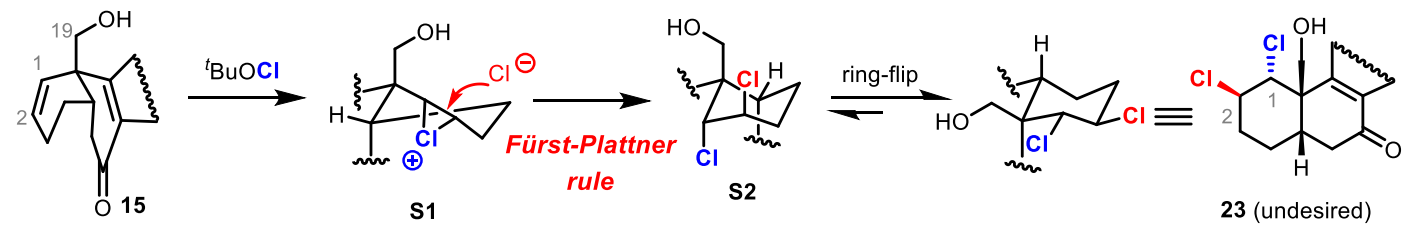

conformation A (pseudo axial for C19)

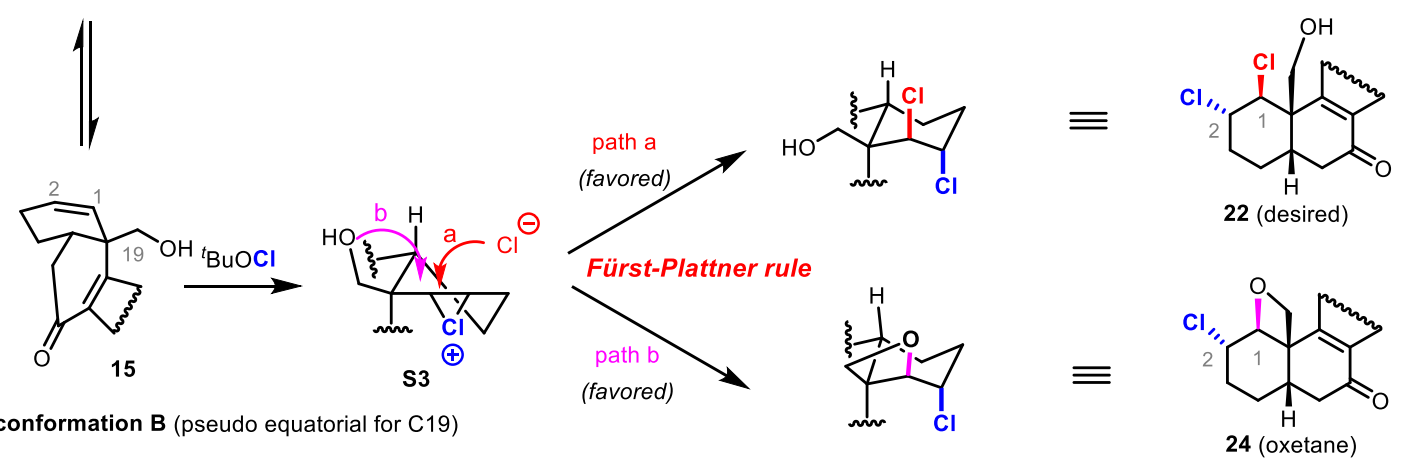

B. Possible pathway for dichlorination of 16 to afford 27 (with conformational lock by sultine):

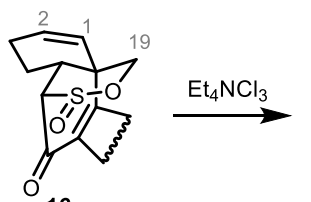

16

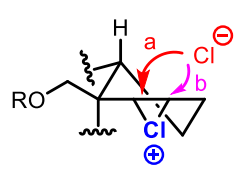

S4

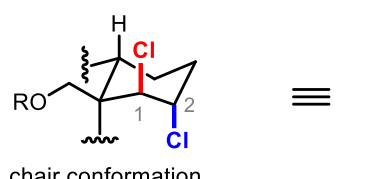

chair conformation

Fürst-Plattner rule (the trans-diaxial effect)

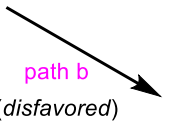

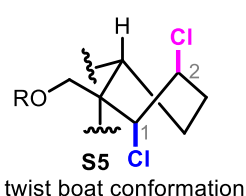

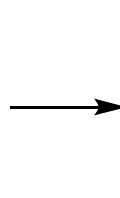

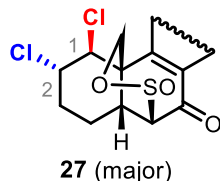

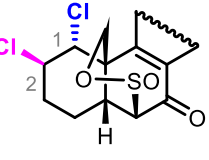

26 (minor) 


\section{Attempted isomerization of the cis-fused CD ring system}

Table S2. Attempted isomerization of compound 14

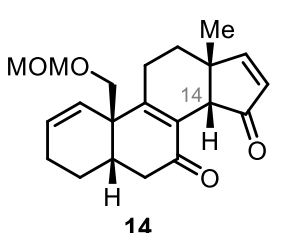

14

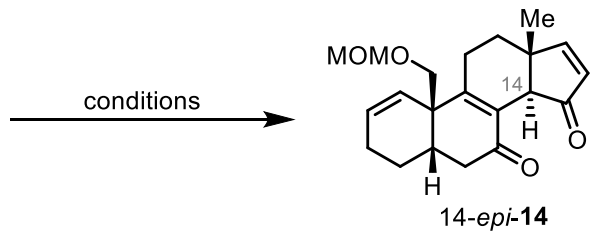

14-epi-14

\begin{tabular}{ccc}
\hline entry & conditions & results \\
\hline 1 & $\mathrm{~K}_{2} \mathrm{CO}_{3}, \mathrm{MeOH}, 60^{\circ} \mathrm{C}$ & no reaction \\
2 & $\mathrm{NaOH}, \mathrm{MeOH}, 60^{\circ} \mathrm{C}$ & no reaction \\
3 & $\mathrm{NaOMe}, \mathrm{MeOH}, 60^{\circ} \mathrm{C}$ & no reaction \\
4 & $\mathrm{DBU}$, toluene & no reaction \\
5 & $\mathrm{LiHMDS}$ then $\mathrm{NH}_{4} \mathrm{Cl}$ & no reaction \\
6 & $\mathrm{LiHMDS}, \mathrm{TMSCl}$ then $\mathrm{CSA}$ & no reaction \\
\hline
\end{tabular}

Table S3. Attempted isomerization of compound S6

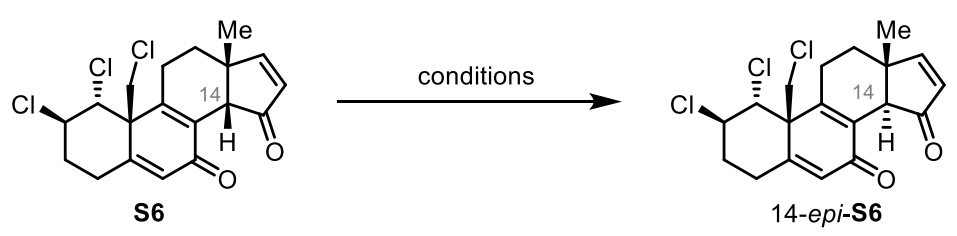

\begin{tabular}{ccc}
\hline entry & conditions & results \\
\hline 1 & $\mathrm{~K}_{2} \mathrm{CO}_{3}, \mathrm{MeOH}, \mathrm{rt}$ to $60^{\circ} \mathrm{C}$ & no reaction \\
2 & $\mathrm{DBU}$, toluene, 100 to $120{ }^{\circ} \mathrm{C}$ & no reaction \\
3 & $\mathrm{NaOMe}, \mathrm{MeOH}, 60{ }^{\circ} \mathrm{C}$ & no reaction \\
4 & $\mathrm{PTSA}$, toluene, 100 to $120^{\circ} \mathrm{C}$ & no reaction \\
5 & $\mathrm{PTSA}, \mathrm{xylene}, 180{ }^{\circ} \mathrm{C}(\mathrm{MW})$ & no reaction \\
7 & $\mathrm{KHMDS}, \mathrm{THF},-78{ }^{\circ} \mathrm{C} ;$ then $\mathrm{CSA}$ & mainly recovered $\mathrm{SM}$ \\
& $\mathrm{KHMDS}, \mathrm{TMSCl}, \mathrm{THF},-78^{\circ} \mathrm{C} ;$ & no reaction \\
\hline
\end{tabular}




\section{Experimental procedures and characterization data for compounds 7-29.}

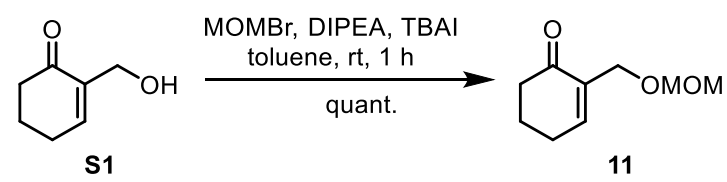

To a solution of alcohol S1 (23.0 g, $183.0 \mathrm{mmol}, 1.0$ equiv) in $360 \mathrm{~mL}$ toluene was sequentially added TBAI (3.4 g, 9.1 mmol, 0.01 equiv), DIPEA (47.6 mL, 274.0 mmol, 1.5 equiv) and $\mathrm{MOMBr}(18.7 \mathrm{~mL}, 219.0 \mathrm{mmol}, 1.2$ equiv) at room temperature. The reaction mixture was stirred at room temperature for $1 \mathrm{~h}$ before it was quenched with $100 \mathrm{~mL} \mathrm{H}_{2} \mathrm{O}$. The resultant mixture was extracted with EtOAc $(3 \times 300 \mathrm{~mL})$, and the combined organic layers were washed with brine $(300 \mathrm{~mL})$, dried over anhydrous $\mathrm{Na}_{2} \mathrm{SO}_{4}$ and filtered. The volatiles were removed under vacuum, and the residue was purified by flash column chromatography with petroleum ether/EtOAc (3:1) to give $\mathbf{1 1}^{8}(32.0 \mathrm{~g}, 100 \%)$ as yellow oil.

Compound 11: TLC (petroleum ether:EtOAc, $2: 1 \mathrm{v} / \mathrm{v}): R_{f}=0.63 ;{ }^{1} \mathrm{H}$ NMR $(400 \mathrm{MHz}$, $\left.\mathrm{CDCl}_{3}\right) \delta 7.03(\mathrm{tt}, J=4.4,1.2 \mathrm{~Hz}, 1 \mathrm{H}), 4.67(\mathrm{~s}, 2 \mathrm{H}), 4.21(\mathrm{q}, J=1.6 \mathrm{~Hz}, 2 \mathrm{H}), 3.38(\mathrm{~s}, 3 \mathrm{H})$, $2.48-2.43(\mathrm{~m}, 2 \mathrm{H}), 2.43-2.38(\mathrm{~m}, 2 \mathrm{H}), 2.02$ (quintet, $J=6.2 \mathrm{~Hz}, 2 \mathrm{H}) ;{ }^{13} \mathrm{C} \mathrm{NMR}(126$ $\left.\mathrm{MHz}_{2} \mathrm{CDCl}_{3}\right) \delta 198.7,147.3,136.3,96.4,64.6,55.5,38.4,25.9,23.0 ; \mathrm{IR}(\mathrm{KBr}): v=2937$, 1672, 1393, 1150, 1097, 1043, $917 \mathrm{~cm}^{-1}$; HRMS (ESI, m/z): calcd for $\mathrm{C}_{9} \mathrm{H}_{15} \mathrm{O}_{3}[\mathrm{M}+\mathrm{H}]^{+}$ 171.1016, found 171.1014.

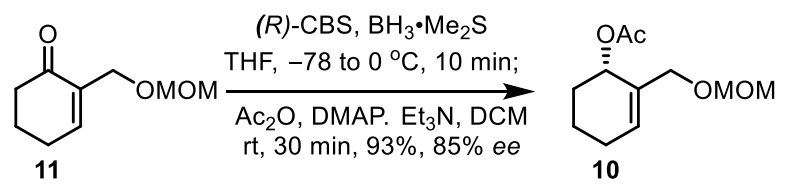

To a solution of 11 (14.0 g, $82.3 \mathrm{mmol}, 1.0$ equiv) in $200 \mathrm{~mL}$ dry toluene was added $(R)$ CBS (1.0 $\mathrm{M}$ in toluene, $16.5 \mathrm{~mL}, 16.5 \mathrm{mmol}, 0.2$ equiv) at $-78^{\circ} \mathrm{C}$, and then $\mathrm{BH}_{3} \cdot \mathrm{Me}_{2} \mathrm{~S}(2.0$ $\mathrm{M}$ in THF, $61.8 \mathrm{~mL}, 123.5 \mathrm{mmol}, 1.5$ equiv) was added dropwise. The solution was stirred at this temperature for $30 \mathrm{~min}$, warmed to $0{ }^{\circ} \mathrm{C}$, and stirred for $10 \mathrm{~min}$. After that, the reaction was quenched with $100 \mathrm{~mL} \mathrm{MeOH}$ slowly at $-78{ }^{\circ} \mathrm{C}$ and warmed to room 
temperature. The solvent was removed under reduced pressure and the crude product was dissolved in $280 \mathrm{~mL} \mathrm{DCM}$. To the resultant solution was added $\mathrm{Et}_{3} \mathrm{~N}(22.9 \mathrm{~mL}, 165.0 \mathrm{mmol}$, 2.0 equiv), DMAP (1.0 g, $8.2 \mathrm{mmol}, 0.1$ equiv) and $\mathrm{Ac}_{2} \mathrm{O}$ (11.7 mL, $123.5 \mathrm{mmol}, 1.5$ equiv) at $0{ }^{\circ} \mathrm{C}$. After stirred at room temperature for $30 \mathrm{~min}$, the reaction mixture was quenched with sat. aq. $\mathrm{NaHCO}_{3}(100 \mathrm{~mL})$ and extracted with ethyl acetate $(3 \times 200 \mathrm{~mL})$. The combined organic layers were washed with brine $(200 \mathrm{~mL})$, dried over anhydrous $\mathrm{Na}_{2} \mathrm{SO}_{4}$, filtered, and concentrated under vacuum. The residue was subjected to flash column chromatography for purification using petroleum ether/EtOAc (15:1) as eluent to give $\mathbf{1 0}$ $(16.4 \mathrm{~g}, 93 \%, 85 \%$ ee) as colorless oil.

Compound 10: TLC (petroleum ether:EtOAc, 5:1 v/v): $R_{f}=0.48$; $[\alpha]_{\mathrm{D}}^{25}-65.9(c 0.06$, $\left.\mathrm{CHCl}_{3}\right) ;{ }^{1} \mathrm{H} \mathrm{NMR}\left(400 \mathrm{MHz}, \mathrm{CDCl}_{3}\right) \delta 6.04(\mathrm{t}, J=3.8 \mathrm{~Hz}, 1 \mathrm{H}), 5.43(\mathrm{t}, J=4.0 \mathrm{~Hz}, 1 \mathrm{H})$, $4.62(\mathrm{~d}, J=6.5 \mathrm{~Hz}, 1 \mathrm{H}), 4.58(\mathrm{~d}, J=6.5 \mathrm{~Hz}, 1 \mathrm{H}), 4.01(\mathrm{~d}, J=12.0 \mathrm{~Hz}, 1 \mathrm{H}), 3.91(\mathrm{~d}, J=$ $11.6 \mathrm{~Hz}, 1 \mathrm{H}), 3.35$ (s, 3H), $2.21-1.98(\mathrm{~m}, 2 \mathrm{H}), 2.05$ (s, 3H), $1.90-1.72(\mathrm{~m}, 2 \mathrm{H}), 1.73-$ $1.56(\mathrm{~m}, 2 \mathrm{H}) ;{ }^{13} \mathrm{C} \mathrm{NMR}\left(101 \mathrm{MHz}, \mathrm{CDCl}_{3}\right) \delta$ 170.9, 132.6, 132.1, 95.9, 68.9, 67.2, 55.4, 28.7, 25.1, 21.5, 18.0; IR (KBr): $v=2939,1732,1440,1371,1239,1149,1044,923 \mathrm{~cm}^{-1}$; HRMS (ESI, $m / z$ ): calcd for $\mathrm{C}_{11} \mathrm{H}_{18} \mathrm{NaO}_{4}[\mathrm{M}+\mathrm{Na}]^{+} 237.1097$, found 237.1097.

HPLC: DAICEL CHIRALPAK IE, hexane/i-PrOH $=24.5 / 1$, flow rate: $1.0 \mathrm{~mL} / \mathrm{min}, \lambda=$ $207 \mathrm{~nm}, \mathrm{t}_{\mathrm{R}}($ minor $)=8.2 \mathrm{~min}, \mathrm{t}_{\mathrm{R}}($ major $)=8.9 \mathrm{~min}, 85 \%$ ee.

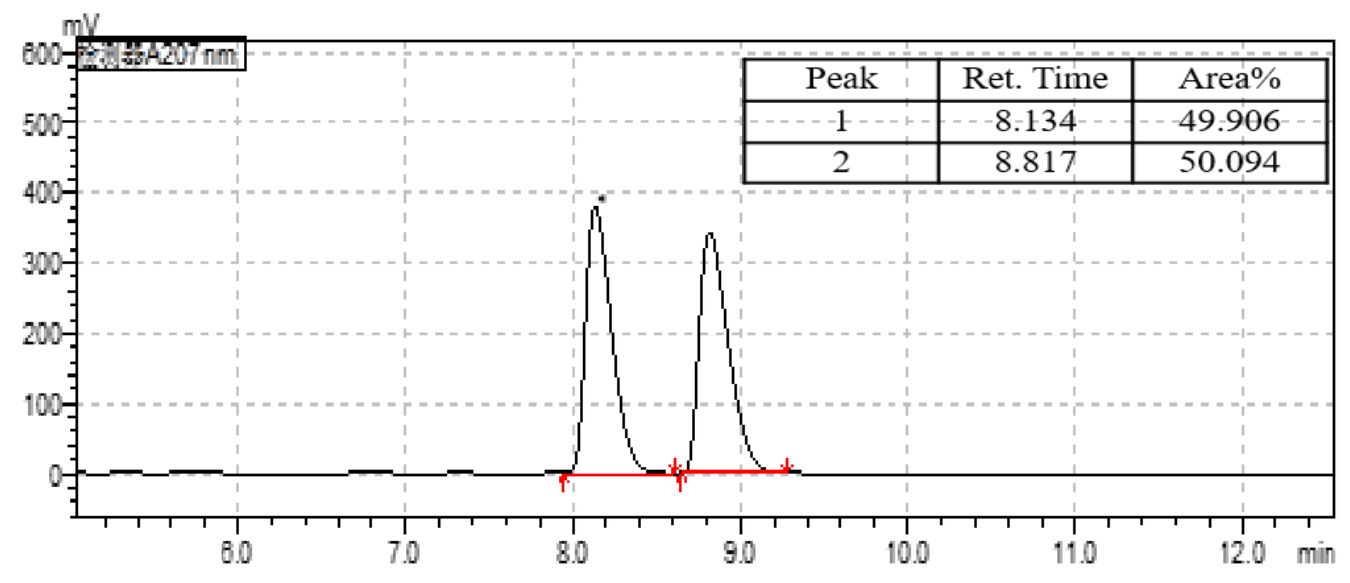



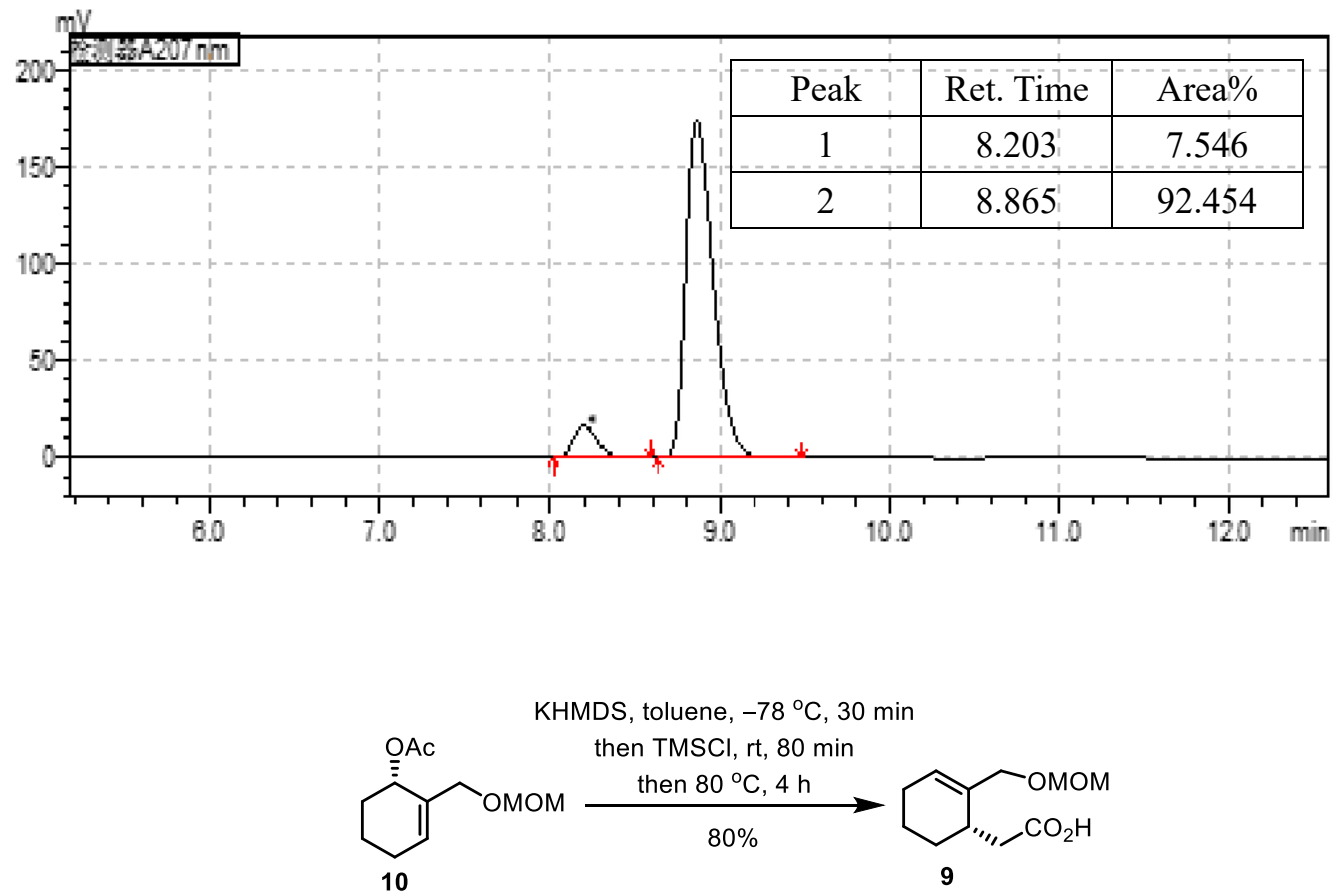

To a solution of 10 (10.0 g, $46.7 \mathrm{mmol}, 1.0$ equiv) in $650 \mathrm{~mL}$ toluene was added KHMDS (1.0 $\mathrm{M}$ in THF, $93.4 \mathrm{~mL}, 93.4 \mathrm{mmol}, 2.0$ equiv) slowly at $-78{ }^{\circ} \mathrm{C}$. After stirring at this temperature for $30 \mathrm{~min}$, TMSCl (14.8 mL, $116.8 \mathrm{mmol}, 2.5$ equiv) was added. After warmed to room temperature and stirred for another $80 \mathrm{~min}$, the reaction mixture was heated at $80^{\circ} \mathrm{C}$ for $4 \mathrm{~h}$ before it was quenched with $0.5 \mathrm{~N}$ aq. $\mathrm{HCl}(250 \mathrm{~mL})$. The resultant mixture was extracted with EtOAc $(3 \times 500 \mathrm{~mL})$, and the combined organic layers were washed with brine $(200 \mathrm{~mL})$, dried over anhydrous $\mathrm{Na}_{2} \mathrm{SO}_{4}$ and filtered. The volatiles were removed under vacuum, and the residue was purified by flash column chromatography with petroleum ether/EtOAc $(10: 1 \rightarrow 2: 1)$ to give acid $9(8.0 \mathrm{~g}, 80 \%)$ as colorless oil.

Compound 9: TLC (petroleum ether:EtOAc, 10:1 v/v): $R_{f}=0.35 ;[\alpha]_{\mathrm{D}}^{25}-1.3(c$ 1.57, $\left.\mathrm{CHCl}_{3}\right) ;{ }^{1} \mathrm{H} \mathrm{NMR}\left(400 \mathrm{MHz}, \mathrm{CDCl}_{3}\right) \delta 5.79(\mathrm{t}, J=3.9 \mathrm{~Hz}, 1 \mathrm{H}), 4.63(\mathrm{~d}, J=6.4 \mathrm{~Hz}, 1 \mathrm{H})$, 4.59 (d, $J=6.4 \mathrm{~Hz}, 1 \mathrm{H}), 4.04$ (d, $J=11.6 \mathrm{~Hz}, 1 \mathrm{H}), 3.90$ (d, $J=11.6 \mathrm{~Hz}, 1 \mathrm{H}), 3.37$ (s, 3H), $2.75-2.63$ (m, 2H), 2.29 (dd, $J=16.0,10.8 \mathrm{~Hz}, 1 \mathrm{H}), 2.03$ (br s, $2 \mathrm{H}), 1.80-1.68(\mathrm{~m}, 1 \mathrm{H})$, $1.64-1.52(\mathrm{~m}, 3 \mathrm{H}) ;{ }^{13} \mathrm{C} \mathrm{NMR}\left(101 \mathrm{MHz}, \mathrm{CDCl}_{3}\right) \delta 179.0,135.5,129.0,95.5,70.6,55.5$, 
$37.8,32.0,27.8,25.3,18.8$; IR (KBr): $v=2940,1733,1371,1240,1149,1044,923,855$ $\mathrm{cm}^{-1}$; HRMS (ESI, $\left.m / z\right)$ : calcd for $\mathrm{C}_{11} \mathrm{H}_{18} \mathrm{NaO}_{4}[\mathrm{M}+\mathrm{Na}]^{+}$237.1097, found 237.1096.

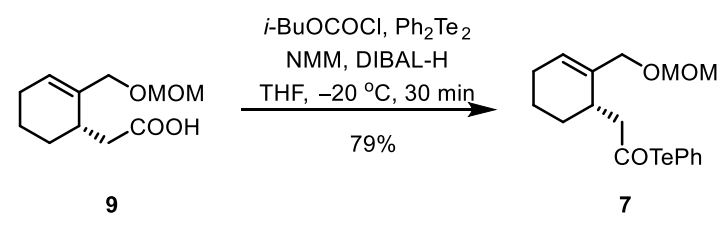

A solution of 9 ( $8.0 \mathrm{~g}, 37.4 \mathrm{mmol}, 1.0$ equiv) in $325 \mathrm{~mL}$ THF was degassed by a flow of Ar for $15 \mathrm{~min}$ and then NMM (4.9 mL, $44.9 \mathrm{mmol}, 1.2$ equiv) and $i$ - $\mathrm{BuOCOCl}(5.8 \mathrm{~mL}$, $44.9 \mathrm{mmol}, 1.2$ equiv) were added at $0{ }^{\circ} \mathrm{C}$. The slurry was stirred at this temperature for 15 min. To another solution of $\mathrm{Ph}_{2} \mathrm{Te}_{2}(10.0 \mathrm{~g}, 24.2 \mathrm{mmol}, 0.65$ equiv) in $325 \mathrm{~mL}$ THF was added DIBAL-H (1.5 M in THF, $35.0 \mathrm{~mL}, 35.0 \mathrm{mmol}, 1.4$ equiv) slowly at $-20{ }^{\circ} \mathrm{C}$ until the color turned from brownish red to colorless. After $5 \mathrm{~min}$, the slurry was transferred to the above colorless solution at $-20{ }^{\circ} \mathrm{C}$, which turned into pale yellow. After stirring at $20{ }^{\circ} \mathrm{C}$ for $30 \mathrm{~min}, 100-200$ mesh silica gel $(20 \mathrm{~g})$ was added. The volatiles were removed under vacuum, and the residue was purified by flash column chromatography with petroleum ether/EtOAc $(200: 1 \rightarrow 20: 1)$ to give compound $7(12.0 \mathrm{~g}, 79 \%)$ as brownish red oil.

Compound 7: TLC (petroleum ether:EtOAc, 10:1 v/v): $R_{f}=0.58$; $[\alpha]_{\mathrm{D}}^{25}+13.8(c 3.00$, $\left.\mathrm{CHCl}_{3}\right) ;{ }^{1} \mathrm{H} \mathrm{NMR}\left(400 \mathrm{MHz}, \mathrm{CDCl}_{3}\right) \delta 7.80-7.70(\mathrm{~m}, 2 \mathrm{H}), 7.41-7.36(\mathrm{~m}, 1 \mathrm{H}), 7.35-$ 7.29 (m, 2H), 5.79 (t, $J=3.2 \mathrm{~Hz}, 1 \mathrm{H}), 4.62(\mathrm{~d}, J=6.4 \mathrm{~Hz}, 1 \mathrm{H}), 4.59$ (d, $J=6.4 \mathrm{~Hz}, 1 \mathrm{H})$, 3.97 (d, $J=11.2 \mathrm{~Hz}, 1 \mathrm{H}), 3.86$ (d, $J=11.2 \mathrm{~Hz}, 1 \mathrm{H}), 3.38(\mathrm{~s}, 3 \mathrm{H}), 2.98(\mathrm{~d}, J=12.0 \mathrm{~Hz}, 1 \mathrm{H})$, $2.82-2.66(\mathrm{~m}, 2 \mathrm{H}), 2.03(\mathrm{q}, J=5.5 \mathrm{~Hz}, 2 \mathrm{H}), 1.80-1.70(\mathrm{~m}, 1 \mathrm{H}), 1.69-1.52(\mathrm{~m}, 3 \mathrm{H})$; ${ }^{13} \mathrm{C}$ NMR $\left(126 \mathrm{MHz}, \mathrm{CDCl}_{3}\right) \delta 201.9,140.1,135.2,129.7,129.0,128.9,114.4,95.6,70.5$, 58.2, 55.6, 32.5, 27.5, 25.2, 18.8; IR (KBr): $v=3051,2940,2867,1694,1172,1009,975$, $734 \mathrm{~cm}^{-1}$; HRMS (DART, $\mathrm{m} / z$ ): calcd for $\mathrm{C}_{17} \mathrm{H}_{26} \mathrm{NO}_{3}{ }^{130} \mathrm{Te}\left[\mathrm{M}+\mathrm{NH}_{4}\right]^{+} 422.0969$, found 422.0969; HRMS (DART, $m / z$ ): calcd for $\mathrm{C}_{17} \mathrm{H}_{26} \mathrm{NO}_{3}{ }^{128} \mathrm{Te}\left[\mathrm{M}+\mathrm{NH}_{4}\right]^{+} 420.0952$, found 420.0952 . 


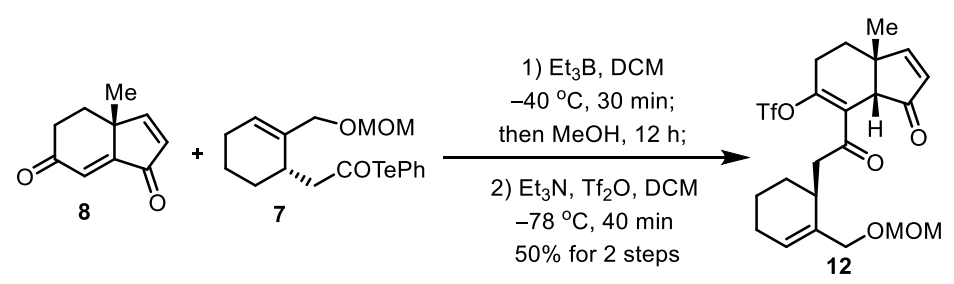

To a solution of 8 (50.0 mg, $0.31 \mathrm{mmol}, 1.0$ equiv) and 7 (167.0 mg, $0.34 \mathrm{mmol}, 1.1$ equiv) in $25 \mathrm{~mL}$ DCM was added $\mathrm{Et}_{3} \mathrm{~B}\left(1.0 \mathrm{M}\right.$ in THF, $0.93 \mathrm{~mL}, 0.93 \mathrm{mmol}, 3.0$ equiv) at $-40{ }^{\circ} \mathrm{C}$. After the mixture was stirred at this temperature for $30 \mathrm{~min}, \mathrm{MeOH}(50 \mathrm{~mL})$ was added. The reaction mixture was allowed to warm to room temperature and stirred for $12 \mathrm{~h}$. After that, the solvent was removed under vacuum and $\mathrm{H}_{2} \mathrm{O}(20 \mathrm{~mL})$ was added. The mixture was then extracted with EtOAc $(3 \times 20 \mathrm{~mL})$ and the combined organic layers were washed with brine $(20 \mathrm{~mL})$, dried over anhydrous $\mathrm{Na}_{2} \mathrm{SO}_{4}$ and concentrated in vacuo. The residue was used directly in the next step.

To the above residue in $9 \mathrm{~mL}$ DCM was added $\mathrm{Et}_{3} \mathrm{~N}(0.26 \mathrm{~mL}, 0.92 \mathrm{mmol}, 3.0$ equiv) and $\mathrm{Tf}_{2} \mathrm{O}(0.21 \mathrm{~mL}, 0.62 \mathrm{mmol}, 2.0$ equiv $)$ at $-78^{\circ} \mathrm{C}$. The solution was stirred for $40 \mathrm{~min}$ before it was quenched with sat. aq. $\mathrm{NaHCO}_{3}(5 \mathrm{~mL})$. The resultant mixture was extracted with DCM $(3 \times 20 \mathrm{~mL})$. The combined organic layers were washed with brine $(20 \mathrm{~mL})$, dried over anhydrous $\mathrm{Na}_{2} \mathrm{SO}_{4}$ and filtered. The volatiles were removed under vacuum, and the residue was purified by flash column chromatography with petroleum ether/EtOAc (10:1 $\rightarrow 8: 1)$ to give enol triflate $\mathbf{1 2}(74.0 \mathrm{mg}, 50 \%)$ as yellow oil.

Compound 12: TLC (petroleum ether:EtOAc, 4:1 v/v): $R_{f}=0.25 ;[\alpha]_{\mathrm{D}}^{25}+96.0(c 1.48$, $\left.\mathrm{CHCl}_{3}\right) ;{ }^{1} \mathrm{H} \mathrm{NMR}\left(400 \mathrm{MHz}, \mathrm{CDCl}_{3}\right) \delta 7.48(\mathrm{~d}, J=5.8 \mathrm{~Hz}, 1 \mathrm{H}), 6.09(\mathrm{~d}, J=5.8 \mathrm{~Hz}, 1 \mathrm{H})$, $5.80(\mathrm{t}, J=3.9 \mathrm{~Hz}, 1 \mathrm{H}), 4.63(\mathrm{~d}, J=6.5 \mathrm{~Hz}, 1 \mathrm{H}), 4.60(\mathrm{~d}, J=6.5 \mathrm{~Hz}, 1 \mathrm{H}), 4.02(\mathrm{~d}, J=11.6$ Hz, 1H), 3.89 (d, $J=11.6 \mathrm{~Hz}, 1 \mathrm{H}), 3.46$ (s, 1H), 3.35 (s, 3H), 3.18 (dd, $J=18.6,1.6 \mathrm{~Hz}$, 1H), $2.99-2.91(\mathrm{~m}, 1 \mathrm{H}), 2.86(\mathrm{dd}, J=18.0,10.4 \mathrm{~Hz}, 1 \mathrm{H}), 2.67-2.50(\mathrm{~m}, 1 \mathrm{H}), 2.37$ (dtd, $J=18.1,4.9,1.5 \mathrm{~Hz}, 1 \mathrm{H}), 2.04(\mathrm{q}, J=5.4 \mathrm{~Hz}, 2 \mathrm{H}), 1.91(\mathrm{dt}, J=13.5,4.7 \mathrm{~Hz}, 1 \mathrm{H}), 1.84-$ $1.77(\mathrm{~m}, 1 \mathrm{H}), 1.75-1.57(\mathrm{~m}, 3 \mathrm{H}), 1.48(\mathrm{ddd}, J=13.3,10.2,4.9 \mathrm{~Hz}, 1 \mathrm{H}), 1.29(\mathrm{~s}, 3 \mathrm{H}) ;{ }^{19} \mathrm{~F}$ 
NMR (376 MHz, $\left.\mathrm{CDCl}_{3}\right) \delta-74.0 ;{ }^{13} \mathrm{C}$ NMR (101 MHz, $\left.\mathrm{CDCl}_{3}\right) \delta$ 204.1, 200.1, 170.2, $146.9,136.3,130.8,128.6,128.0,118.2\left(\mathrm{q}, J=320.2 \mathrm{~Hz}, \mathrm{CF}_{3}\right), 95.6,70.5,56.6,55.2,46.1$, 42.9, 34.1, 29.7, 26.7, 25.6, 25.2, 23.0, 18.4; IR (KBr): $v=2931,2874,1715,1421,1248$, 1214, 1142, $617 \mathrm{~cm}^{-1}$; HRMS (ESI, $\mathrm{m} / z$ ): calcd for $\mathrm{C}_{22} \mathrm{H}_{27} \mathrm{~F}_{3} \mathrm{NaO}_{7} \mathrm{~S}[\mathrm{M}+\mathrm{Na}]^{+}$515.1322, found 515.1320 .
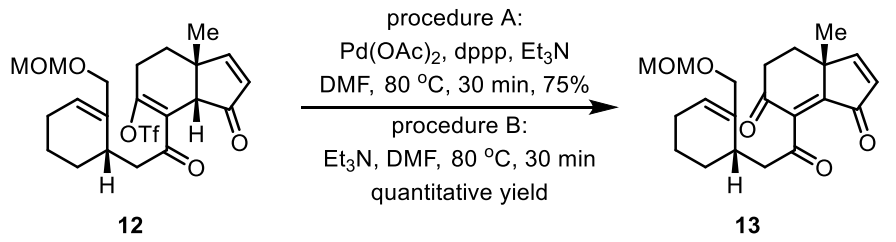

Procedure A: After degassing a mixture of 12 (14.0 mg, $0.028 \mathrm{mmol}, 1.0$ equiv), $\mathrm{Pd}(\mathrm{OAc})_{2}\left(2.6 \mathrm{mg}, 0.011 \mathrm{mmol}, 0.4\right.$ equiv), dppp ( $4.7 \mathrm{mg}, 0.011 \mathrm{mmol}, 0.4$ equiv) and $\mathrm{Et}_{3} \mathrm{~N}$ ( $0.42 \mathrm{~mL}, 0.14 \mathrm{mmol}, 5.0$ equiv) in $1.4 \mathrm{~mL}$ DMF, the reaction mixture was stirred at $80{ }^{\circ} \mathrm{C}$ for $30 \mathrm{~min}$. After cooling down to room temperature, $10 \mathrm{~mL} \mathrm{H}_{2} \mathrm{O}$ was added. The resultant mixture was extracted with EtOAc $(3 \times 10 \mathrm{~mL})$. The combined organic layers were washed with brine $(20 \mathrm{~mL})$, dried over anhydrous $\mathrm{Na}_{2} \mathrm{SO}_{4}$ and filtered. The volatiles were removed under vacuum, and the residue was purified by flash column chromatography with petroleum ether/EtOAc (2:1) to give compound 13 (7.6 mg, 75\%) as yellow oil.

Procedure B: To a solution of $\mathbf{1 2}(30.0 \mathrm{mg}, 0.06 \mathrm{mmol}, 1.0$ equiv) in $1.5 \mathrm{~mL}$ DMF was added $\mathrm{Et}_{3} \mathrm{~N}$ (40 $\mu \mathrm{L}, 0.29 \mathrm{mmol}, 4.8$ equiv), and the reaction mixture was stirred at $80{ }^{\circ} \mathrm{C}$ for $30 \mathrm{~min}$. After cooling down to room temperature, $10 \mathrm{~mL} \mathrm{H}_{2} \mathrm{O}$ was added. The resultant mixture was extracted with EtOAc $(3 \times 2 \mathrm{~mL})$. The combined organic layers were washed with brine $(3 \mathrm{~mL})$, dried over anhydrous $\mathrm{Na}_{2} \mathrm{SO}_{4}$ and filtered. The volatiles were removed under vacuum, and the residue was purified by flash column chromatography with petroleum ether/EtOAc (2:1) to give compound $\mathbf{1 3}$ (22.0 mg, quantitative yield) as yellow oil.

Compound 13: TLC (petroleum ether:EtOAc, 1:1 v/v): $R_{f}=0.48$; $[\alpha]_{\mathrm{D}}^{25}+93.6(c 0.75$, $\left.\mathrm{CHCl}_{3}\right) ;{ }^{1} \mathrm{H} \mathrm{NMR}\left(400 \mathrm{MHz}, \mathrm{CDCl}_{3}\right) \delta 7.58(\mathrm{~d}, J=6.0 \mathrm{~Hz}, 1 \mathrm{H}), 6.29(\mathrm{~d}, J=6.0 \mathrm{~Hz}, 1 \mathrm{H})$, 
$5.78(\mathrm{t}, J=4.0 \mathrm{~Hz}, 1 \mathrm{H}), 4.64(\mathrm{~d}, J=6.8 \mathrm{~Hz}, 1 \mathrm{H}), 4.62(\mathrm{~d}, J=6.8 \mathrm{~Hz}, 1 \mathrm{H}), 4.01$ (d, $J=7.6$ Hz, 1H), 3.89 (d, $J=7.6 \mathrm{~Hz}, 1 \mathrm{H}), 3.37$ (s, 3H), $2.96-2.87$ (m, 2H), 2.79 (ddd, $J=19.2$, 12.4, $6.8 \mathrm{~Hz}, 1 \mathrm{H}), 2.67-2.63(\mathrm{~m}, 2 \mathrm{H}), 2.63-2.57(\mathrm{~m}, 2 \mathrm{H}), 2.13-2.05(\mathrm{~m}, 2 \mathrm{H}), 1.80-$ $1.73(\mathrm{~m}, 2 \mathrm{H}), 1.68-1.54(\mathrm{~m}, 2 \mathrm{H}), 1.42(\mathrm{~s}, 3 \mathrm{H}) ;{ }^{13} \mathrm{C} \mathrm{NMR}\left(101 \mathrm{MHz}, \mathrm{CDCl}_{3}\right) \delta 202.5$, 197.0, 194.7, 166.5, 152.1, 136.4, 135.3, 133.3, 128.1, 95.9, 70.6, 55.5, 47.3, 42.9, 34.6, 31.6, 29.5, 27.3, 25.3, 23.9, 18.6; IR (KBr): $v=2957,2922,2868,1708,1673,1446,1163$, $735 \mathrm{~cm}^{-1}$; HRMS (ESI, $m / z$ ): calcd for $\mathrm{C}_{21} \mathrm{H}_{26} \mathrm{NaO}_{5}[\mathrm{M}+\mathrm{Na}]^{+} 381.1672$; found, 381.1670 .

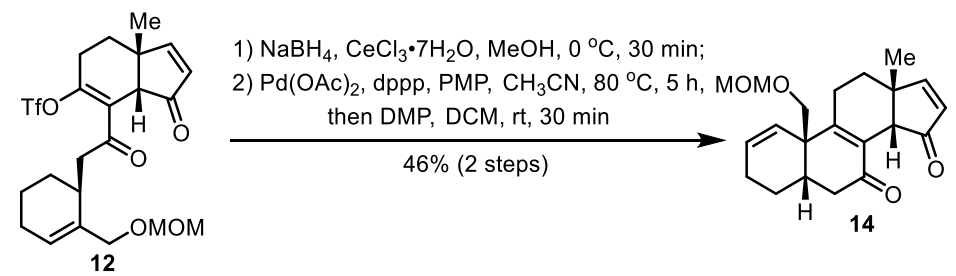

To a solution of $12(80.0 \mathrm{mg}, 0.16 \mathrm{mmol}, 1.0$ equiv) in $2.0 \mathrm{~mL} \mathrm{MeOH}$ was added $\mathrm{CeCl}_{3} \cdot 7 \mathrm{H}_{2} \mathrm{O}\left(178.0 \mathrm{mg}, 0.48 \mathrm{mmol}, 3.0\right.$ equiv) at $0{ }^{\circ} \mathrm{C}$. After complete dissolution of $\mathrm{CeCl}_{3} \cdot 7 \mathrm{H}_{2} \mathrm{O}, \mathrm{NaBH}_{4}(18.0 \mathrm{mg}, 0.48 \mathrm{mmol}, 3.0$ equiv $)$ was added at the same temperature. The solution was stirred for 30 min before it was quenched with $\mathrm{H}_{2} \mathrm{O}(10 \mathrm{~mL})$. Then $\mathrm{MeOH}$ was removed under vacuum. The resultant mixture was extracted with EtOAc $(3 \times 15 \mathrm{~mL})$. The combined organic layers were washed with brine $(15 \mathrm{~mL})$, dried over anhydrous $\mathrm{Na}_{2} \mathrm{SO}_{4}$ and filtered. The volatiles were removed under vacuum, and the residue was purified by flash column chromatography with petroleum ether/EtOAc (6:1) to give a mixture of major diastereomers $(54.0 \mathrm{mg})$ as yellow oil.

After degassing a solution of major diastereomers (54.0 mg, $0.11 \mathrm{mmol}, 1.0$ equiv), $\mathrm{Pd}(\mathrm{OAc})_{2}(5.0 \mathrm{mg}, 0.022 \mathrm{mmol}, 0.2$ equiv), dppp (9.0 $\mathrm{mg}, 0.022 \mathrm{mmol}, 0.2$ equiv) and PMP $(40.0 \mu \mathrm{L}, 0.22 \mathrm{mmol}, 2.0$ equiv) in $3.0 \mathrm{~mL} \mathrm{MeCN}$, the reaction mixture was stirred at $80{ }^{\circ} \mathrm{C}$ for $5 \mathrm{~h}$. After cooling down to room temperature, 5.0 mL DCM and DMP (233.0 $\mathrm{mg}, 0.55 \mathrm{mmol}, 5.0$ equiv) were added. After stirring at room temperature for $0.5 \mathrm{~h}$, the reaction mixture was quenched with sat. aq. $\mathrm{Na}_{2} \mathrm{SO}_{3}(10 \mathrm{~mL})$ and extracted with $\mathrm{DCM}$ 
$(3 \times 20 \mathrm{~mL})$. The combined organic layers were washed with brine $(20 \mathrm{~mL})$, dried over anhydrous $\mathrm{Na}_{2} \mathrm{SO}_{4}$ and filtered. The volatiles were removed under vacuum, and the residue was purified by flash column chromatography with petroleum ether/EtOAc $(7: 1 \rightarrow 6: 1 \rightarrow$ 5:1) to give compound $\mathbf{1 4}$ ( $25.0 \mathrm{mg}, 46 \%$ for 2 steps) as yellow oil.

Compound 14: TLC (petroleum ether:EtOAc, 2:1 v/v): $R_{f}=0.31$; $[\alpha]_{\mathrm{D}}^{25}+183.8(c$ 1.10, $\left.\mathrm{CHCl}_{3}\right) ;{ }^{1} \mathrm{H} \mathrm{NMR}\left(400 \mathrm{MHz}, \mathrm{CDCl}_{3}\right) \delta 7.32(\mathrm{~d}, J=5.6 \mathrm{~Hz}, 1 \mathrm{H}), 6.20(\mathrm{~d}, J=5.6 \mathrm{~Hz}, 1 \mathrm{H})$, $5.86(\mathrm{dt}, J=10.0,3.6 \mathrm{~Hz}, 1 \mathrm{H}), 5.58(\mathrm{dt}, J=10.0,2.2 \mathrm{~Hz}, 1 \mathrm{H}), 4.61(\mathrm{~d}, J=6.4 \mathrm{~Hz}, 1 \mathrm{H})$, 4.59 (d, $J=6.4 \mathrm{~Hz}, 1 \mathrm{H}), 3.66$ (s, 1H), 3.64 (d, $J=9.6 \mathrm{~Hz}, 1 \mathrm{H}), 3.56$ (d, $J=9.6 \mathrm{~Hz}, 1 \mathrm{H})$, 3.34 (s, 3H), 2.88 (dd, $J=16.8,5.2 \mathrm{~Hz}, 1 \mathrm{H}), 2.45$ (dd, $J=16.8,4.4 \mathrm{~Hz}, 1 \mathrm{H}), 2.34$ (dt, $J=$ 13.2, $4.2 \mathrm{~Hz}, 1 \mathrm{H}), 2.25$ (ddd, $J=16.4,4.4,2.7 \mathrm{~Hz}, 1 \mathrm{H}), 2.10-2.08$ (m, 2H), 1.74 (dt, $J=$ 12.8, $4.0 \mathrm{~Hz}, 2 \mathrm{H}), 1.69-1.56(\mathrm{~m}, 2 \mathrm{H}), 1.40(\mathrm{td}, J=13.2,4.4 \mathrm{~Hz}, 1 \mathrm{H}), 1.28(\mathrm{~s}, 3 \mathrm{H}) ;{ }^{13} \mathrm{C}$ NMR (101 MHz, $\left.\mathrm{CDCl}_{3}\right) \delta 206.7,196.3,170.4,159.5,133.9,131.3,130.5,127.6,96.9$, $72.2,55.6,50.3,45.4,44.9,40.7,34.9,34.5,27.1,25.3,24.6,24.3$; IR (KBr): $v=3020$, 2926, 1709, 1586, 1667, 1375, 1152, $1042 \mathrm{~cm}^{-1}$; HRMS (ESI, $\mathrm{m} / z$ ): calcd for $\mathrm{C}_{21} \mathrm{H}_{27} \mathrm{O}_{4}$ $[\mathrm{M}+\mathrm{H}]^{+}$343.1904, found 343.1898.

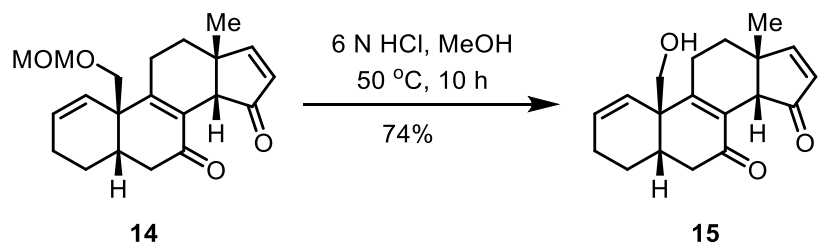

To a solution of 14 (700.0 mg, $2.0 \mathrm{mmol}, 1.0$ equiv) in $20 \mathrm{~mL} \mathrm{MeOH}$ was added $6 \mathrm{~N} \mathrm{HCl}$ (3.2 $\mathrm{mL}, 20.0 \mathrm{mmol}, 10.0$ equiv), and the reaction mixture was stirred at $50{ }^{\circ} \mathrm{C}$ overnight. After cooling to room temperature, the reaction mixture was quenched with sat. aq. $\mathrm{NaHCO}_{3}(50 \mathrm{~mL})$, extracted with EtOAc $(3 \times 200 \mathrm{~mL})$. The combined organic layers were washed with brine $(100 \mathrm{~mL})$, dried over anhydrous $\mathrm{Na}_{2} \mathrm{SO}_{4}$ and filtered. The volatiles were removed under vacuum, and the residue was purified by flash column chromatography with petroleum ether/acetone $(5: 1 \rightarrow 4: 1)$ to give compound $7(442.0 \mathrm{mg}, 74 \%)$ as yellow oil. 
Compound 15: TLC (petroleum ether:acetone, 3:1 v/v): $R_{f}=0.35 ;[\alpha]_{\mathrm{D}}^{25}+203.5(c 0.43$, $\left.\mathrm{CHCl}_{3}\right) ;{ }^{1} \mathrm{H} \mathrm{NMR}\left(400 \mathrm{MHz}, \mathrm{CDCl}_{3}\right) \delta 7.32(\mathrm{~d}, J=5.6 \mathrm{~Hz}, 1 \mathrm{H}), 6.20(\mathrm{~d}, J=5.6 \mathrm{~Hz}, 1 \mathrm{H})$, $5.95(\mathrm{dt}, J=10.0,3.6 \mathrm{~Hz}, 1 \mathrm{H}), 5.57(\mathrm{dt}, J=10.0,2.0 \mathrm{~Hz}, 1 \mathrm{H}), 3.74(\mathrm{~d}, J=11.2 \mathrm{~Hz}, 1 \mathrm{H})$, $3.67(\mathrm{~d}, J=11.2 \mathrm{~Hz}, 1 \mathrm{H}), 3.66(\mathrm{~s}, 1 \mathrm{H}), 2.88(\mathrm{dd}, J=17.2,5.6 \mathrm{~Hz}, 1 \mathrm{H}), 2.44(\mathrm{dd}, J=17.2$, $3.6 \mathrm{~Hz}, 1 \mathrm{H}), 2.36(\mathrm{dt}, J=10.6,4.6 \mathrm{~Hz}, 1 \mathrm{H}), 2.28-2.19(\mathrm{~m}, 1 \mathrm{H}), 2.09-1.99(\mathrm{~m}, 2 \mathrm{H}), 1.77$ $(\mathrm{dt}, J=13.6,3.6 \mathrm{~Hz}, 2 \mathrm{H}), 1.73-1.58(\mathrm{~m}, 3 \mathrm{H}), 1.38(\mathrm{td}, J=14.8,3.3 \mathrm{~Hz}, 1 \mathrm{H}), 1.28(\mathrm{~s}, 3 \mathrm{H})$; ${ }^{13} \mathrm{C} \mathrm{NMR}\left(101 \mathrm{MHz}, \mathrm{CDCl}_{3}\right) \delta 206.7,196.0,170.4,159.4,134.0,133.3,130.8,127.1,66.3$, 50.2, 46.7, 45.4, 40.5, 34.4, 33.9, 27.2, 25.2, 24.9, 24.3; IR (KBr): $v=3458,3019,2924$, 1708, 1662, 1376, 1238, $754 \mathrm{~cm}^{-1}$; HRMS (DART, $m / z$ ): calcd for $\mathrm{C}_{19} \mathrm{H}_{23} \mathrm{O}_{3}[\mathrm{M}+\mathrm{H}]^{+}$ 299.1642 , found 299.1641.

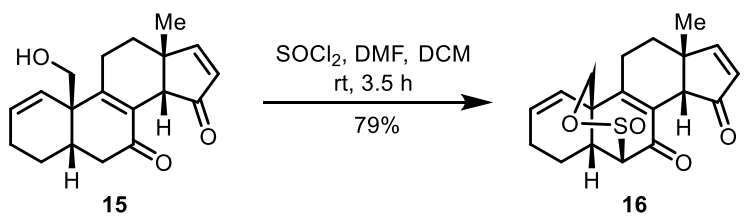

To a solution of 15 (400.0 mg, $1.34 \mathrm{mmol}, 1.0$ equiv) in $13.0 \mathrm{~mL}$ DCM was added DMF (1.0 mL, $13.4 \mathrm{mmol}, 10.0$ equiv) at room temperature, and then $\mathrm{SOCl}_{2}(292.0 \mu \mathrm{L}, 4.02$ mmol, 3.0 equiv) was added slowly at $0{ }^{\circ} \mathrm{C}$. After stirring for $30 \mathrm{~min}$, the reaction mixture was warmed to room temperature and stirred for another $3.5 \mathrm{~h}$. The reaction mixture was quenched with $\mathrm{H}_{2} \mathrm{O}(50 \mathrm{~mL})$ and extracted with DCM $(3 \times 100 \mathrm{~mL})$. The combined organic layers were washed with brine $(100 \mathrm{~mL})$, dried over anhydrous $\mathrm{Na}_{2} \mathrm{SO}_{4}$ and filtered. The volatiles were removed under vacuum, and the residue was purified by flash column chromatography with petroleum ether/acetone $(7: 1 \rightarrow 6: 1)$ to give compound $\mathbf{1 6}(367.0 \mathrm{mg}$, $79 \%$ ) as a yellow solid.

Compound 16: mp: $185.3-187.9{ }^{\circ} \mathrm{C}$; TLC (petroleum ether:EtOAc, $2: 1 \mathrm{v} / \mathrm{v}$ ): $R_{f}=0.41$; $[\alpha]_{\mathrm{D}}^{25}+177.5\left(c 0.79, \mathrm{CHCl}_{3}\right) ;{ }^{1} \mathrm{H} \mathrm{NMR}\left(400 \mathrm{MHz}, \mathrm{CDCl}_{3}\right) \delta 7.37(\mathrm{~d}, J=5.6 \mathrm{~Hz}, 1 \mathrm{H}), 6.23$ $(\mathrm{d}, J=5.6 \mathrm{~Hz}, 1 \mathrm{H}), 5.98(\mathrm{ddd}, J=10.0,4.0,2.4 \mathrm{~Hz}, 1 \mathrm{H}), 5.39(\mathrm{dt}, J=10.0,2.3 \mathrm{~Hz}, 1 \mathrm{H})$, $4.33(\mathrm{~d}, J=11.2 \mathrm{~Hz}, 1 \mathrm{H}), 3.71(\mathrm{~s}, 1 \mathrm{H}), 3.53(\mathrm{~d}, J=11.2 \mathrm{~Hz}, 1 \mathrm{H}), 3.42(\mathrm{~d}, J=1.6 \mathrm{~Hz}, 1 \mathrm{H})$, 
$3.09(\mathrm{dt}, J=12.7,2.5 \mathrm{~Hz}, 1 \mathrm{H}), 2.31-2.19(\mathrm{~m}, 1 \mathrm{H}), 2.16-2.07(\mathrm{~m}, 2 \mathrm{H}), 1.93-1.81(\mathrm{~m}$, $2 \mathrm{H}), 1.74-1.62(\mathrm{~m}, 1 \mathrm{H}), 1.54-1.38(\mathrm{~m}, 2 \mathrm{H}), 1.34(\mathrm{~s}, 3 \mathrm{H}) ;{ }^{13} \mathrm{C} \mathrm{NMR}\left(101 \mathrm{MHz}, \mathrm{CDCl}_{3}\right)$ $\delta$ 206.1, 187.8, 170.8, 158.0, 134.1, 133.9, 132.0, 123.8, 71.5, 61.6, 49.8, 45.8, 41.3, 34.0, 32.3, 27.6, 25.4, 24.4, 23.1; IR (KBr): $v=2960,2930,1707,1655,1618,1457,1137,803$ $\mathrm{cm}^{-1}$; HRMS (ESI, $m / z$ ): calcd for $\mathrm{C}_{19} \mathrm{H}_{21} \mathrm{O}_{4} \mathrm{~S}[\mathrm{M}+\mathrm{H}]^{+} 345.1155$, found 345.1155 .

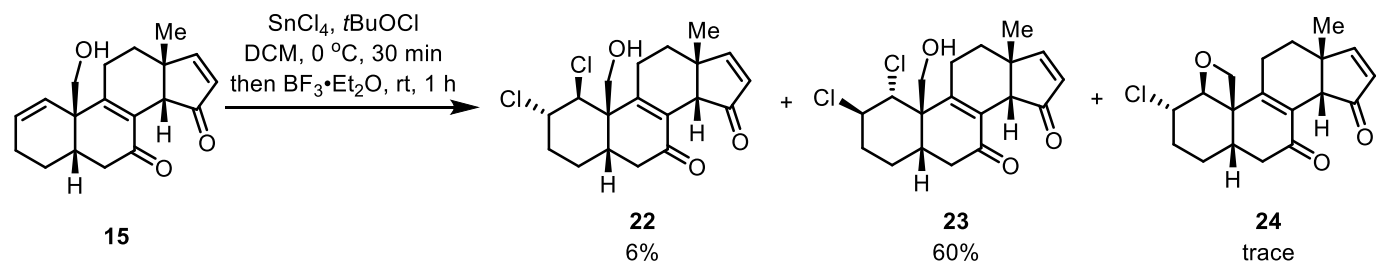

To a solution of 15 ( $93.0 \mathrm{mg}, 0.31 \mathrm{mmol}, 1.0$ equiv) in $3.3 \mathrm{~mL}$ DCM was added a solution of $\mathrm{SnCl}_{4}(1.0 \mathrm{M}$ in $\mathrm{DCM}, 0.93 \mathrm{~mL}, 0.93 \mathrm{mmol}, 3.0$ equiv) at room temperature. The solution was stirred at room temperature for $5 \mathrm{~min}$ before it was cooled to $0{ }^{\circ} \mathrm{C}$. A solution of ${ }^{t} \mathrm{BuOCl}(1.0 \mathrm{M}$ in DCM, $88.0 \mu \mathrm{L}, 0.088 \mathrm{mmol}, 2.5$ equiv) in DCM was then added dropwise. The mixture was allowed to stir at $0{ }^{\circ} \mathrm{C}$ for 30 min before $\mathrm{BF}_{3} \cdot \mathrm{Et}_{2} \mathrm{O}(77.0 \mu \mathrm{L}$, $0.62 \mathrm{mmol}, 2.0$ equiv) was added. After warmed to room temperature and stirred for $1 \mathrm{~h}$, the reaction mixture was quenched with sat. aq. $\mathrm{Na}_{2} \mathrm{SO}_{3}(20 \mathrm{~mL})$ at $0{ }^{\circ} \mathrm{C}$ and extracted with DCM $(3 \times 20 \mathrm{~mL})$. The combined organic layers were washed with brine $(20 \mathrm{~mL})$, dried over anhydrous $\mathrm{Na}_{2} \mathrm{SO}_{4}$ and filtered. The volatiles were removed under vacuum, and the residue was purified by flash column chromatography with petroleum ether/acetone (7:1 $\rightarrow 6: 1 \rightarrow 5: 1)$ to give compound $\mathbf{2 3}(69.0 \mathrm{mg}, 60 \%)$ and compound $22(7.0 \mathrm{mg}, 6 \%)$ as yellow foam.

Compound 22: TLC (petroleum ether:acetone, $3: 1 \mathrm{v} / \mathrm{v}): R_{f}=0.39 ;[\alpha]_{\mathrm{D}}^{25}+131.2(c 0.95$, $\left.\mathrm{CHCl}_{3}\right) ;{ }^{1} \mathrm{H} \mathrm{NMR}\left(600 \mathrm{MHz}, \mathrm{CDCl}_{3}\right) \delta 7.33(\mathrm{~d}, J=5.6 \mathrm{~Hz}, 1 \mathrm{H}), 6.23(\mathrm{~d}, J=5.6 \mathrm{~Hz}, 1 \mathrm{H})$, 4.68 (br s, 1H), 4.31 (br s, 1H), 4.11 (br s, 1H), 3.99 (dd, $J=10.8,5.4 \mathrm{~Hz}, 1 \mathrm{H}), 3.74$ (s, $1 \mathrm{H}), 2.71(\mathrm{~d}, J=16.8 \mathrm{~Hz}, 1 \mathrm{H}), 2.51(\mathrm{dt}, J=16.8,3.0 \mathrm{~Hz}, 1 \mathrm{H}), 2.49-2.40(\mathrm{~m}, 2 \mathrm{H}), 2.37$ (ddt, $J=14.4,10.8,3.6 \mathrm{~Hz}, 1 \mathrm{H}), 2.24-2.12(\mathrm{~m}, 1 \mathrm{H}), 1.98-1.85(\mathrm{~m}, 3 \mathrm{H}), 1.84$ (dt, $J=$ 13.2, $3.6 \mathrm{~Hz}, 1 \mathrm{H}), 1.55$ (br s, $1 \mathrm{H}), 1.42(\mathrm{td}, J=13.2,3.0 \mathrm{~Hz}, 1 \mathrm{H}), 1.30(\mathrm{~s}, 3 \mathrm{H}) ;{ }^{13} \mathrm{C} \mathrm{NMR}$ 
$\left(151 \mathrm{MHz}, \mathrm{CDCl}_{3}, 50{ }^{\circ} \mathrm{C}\right) \delta 205.5,194.7,173.3,161.9,134.5,132.6,69.7,65.0,59.4,50.3$, 47.5, 46.0, 40.0, 35.2, 34.4, 28.4, 27.5, 26.9, 23.9; IR (KBr): $v=3485,2960,2925,1703$, 1664, 1452, 1261, $757 \mathrm{~cm}^{-1}$; HRMS (ESI, $m / z$ ): calcd for $\mathrm{C}_{19} \mathrm{H}_{23} \mathrm{Cl}_{2} \mathrm{O}_{3}[\mathrm{M}+\mathrm{H}]^{+}$369.1019, found 369.1021 .

Compound 23: TLC (petroleum ether:acetone, $3: 1 \mathrm{v} / \mathrm{v}): R_{f}=0.37 ;[\alpha]_{\mathrm{D}}^{25}+43.8(c 0.71$, $\left.\mathrm{CHCl}_{3}\right) ;{ }^{1} \mathrm{H} \mathrm{NMR}\left(400 \mathrm{MHz}, \mathrm{CDCl}_{3}\right) \delta 7.38(\mathrm{~d}, J=5.6 \mathrm{~Hz}, 1 \mathrm{H}), 6.23(\mathrm{~d}, J=5.6 \mathrm{~Hz}, 1 \mathrm{H})$, 4.35 (d, $J=8.4 \mathrm{~Hz}, 1 \mathrm{H}), 4.22-4.08$ (m, 1H), 4.12 (dd, $J=11.6,4.3 \mathrm{~Hz}, 1 \mathrm{H}), 3.74-3.66$ (m, $1 \mathrm{H}), 3.71$ (s, 1H), $3.05-2.55$ (m, 1H), 2.69 (br s, 1H), $3.47-3.36$ (m, 2H), 2.14 (br $\mathrm{s}, 1 \mathrm{H}), 1.98-1.78(\mathrm{~m}, 3 \mathrm{H}), 1.78-1.61(\mathrm{~m}, 3 \mathrm{H}), 1.54-1.40(\mathrm{~m}, 1 \mathrm{H}), 1.27(\mathrm{~s}, 3 \mathrm{H}) ;{ }^{13} \mathrm{C}$ $\operatorname{NMR}\left(151 \mathrm{MHz}, \mathrm{CDCl}_{3}, 50^{\circ} \mathrm{C}\right) \delta 205.9,195.6,170.1,156.0,134.5,133.6,66.6,65.2,60.6$, 51.5, 51.2, 44.5, 40.4, 35.3, 35.1, 26.3, 25.6, 23.4 (one $\mathrm{CH}_{2}$ carbon was not observed); IR (KBr): $v=3479,2963,2926,1703,1666,1453,1059,754 \mathrm{~cm}^{-1}$; HRMS (ESI, $\mathrm{m} / z$ ): calcd for $\mathrm{C}_{19} \mathrm{H}_{23} \mathrm{Cl}_{2} \mathrm{O}_{3}[\mathrm{M}+\mathrm{H}]^{+}$369.1019, found 369.1017.

Compound 24: TLC (petroleum ether:acetone, $3: 1 \mathrm{v} / \mathrm{v}): R_{f}=0.41 ;[\alpha]_{\mathrm{D}}^{25}+80.8(c 0.18$, $\left.\mathrm{CHCl}_{3}\right) ;{ }^{1} \mathrm{H} \mathrm{NMR}\left(400 \mathrm{MHz}, \mathrm{CDCl}_{3}\right) \delta 7.34(\mathrm{~d}, J=5.6 \mathrm{~Hz}, 1 \mathrm{H}), 6.24(\mathrm{~d}, J=5.6 \mathrm{~Hz}, 1 \mathrm{H})$, $4.90(\mathrm{~d}, J=4.8 \mathrm{~Hz}, 1 \mathrm{H}), 4.66(\mathrm{~d}, J=6.0 \mathrm{~Hz}, 1 \mathrm{H}), 4.32$ (d, $J=6.0 \mathrm{~Hz}, 1 \mathrm{H}), 4.27$ (dt, $J=$ 7.6, 4.4 Hz, 1H), 3.63 (s, 1H), $2.79-2.60$ (m, 3H), 2.47 (dd, $J=16.4,2.4 \mathrm{~Hz}, 1 \mathrm{H}), 2.21-$ $2.10(\mathrm{~m}, 1 \mathrm{H}), 2.06-1.94(\mathrm{~m}, 2 \mathrm{H}), 1.76-1.55(\mathrm{~m}, 3 \mathrm{H}), 1.42(\mathrm{td}, J=13.6,3.6 \mathrm{~Hz}, 1 \mathrm{H})$, $1.30(\mathrm{~s}, 3 \mathrm{H}) ;{ }^{13} \mathrm{C} \mathrm{NMR}\left(101 \mathrm{MHz}, \mathrm{CDCl}_{3}\right) \delta 205.8,194.6,170.2,152.5,134.4,132.5,86.7$, 76.5, 57.5, 49.7, 46.7, 45.8, 38.9, 36.2, 34.1, 29.1, 27.8, 24.4, 23.3; IR (KBr): $v=2961$, 2924, 1708, 1672, 1261, 1020, 800, $758 \mathrm{~cm}^{-1}$; HRMS (ESI, $\mathrm{m} / \mathrm{z}$ ): calcd for $\mathrm{C}_{19} \mathrm{H}_{22} \mathrm{ClO}_{3}$ $[\mathrm{M}+\mathrm{H}]^{+}$333.1252, found 333.1251.

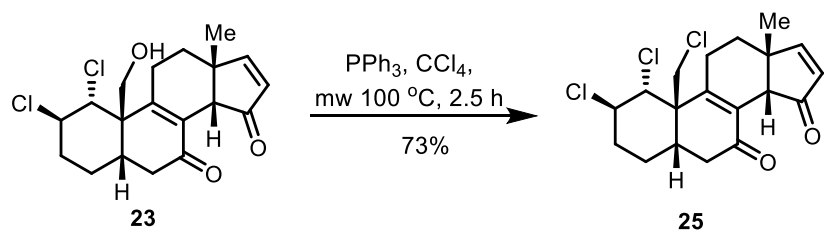

To a microwave vial was added 23 (50.0 mg, $0.14 \mathrm{mmol}, 1.0$ equiv), $\mathrm{PPh}_{3}(107.0 \mathrm{mg}, 0.42$ 
mmol, 3.0 equiv) and $2.5 \mathrm{~mL} \mathrm{CCl}_{4}$. The vial was sealed and heated under microwave irradiation at $100{ }^{\circ} \mathrm{C}$ for $2.5 \mathrm{~h}$. The volatiles were removed under vacuum, and then purified by flash column chromatography with petroleum ether/EtOAc $(7: 1)$ to give compound $\mathbf{2 5}$ (38.0 $\mathrm{mg}, 73 \%)$ as a yellow solid.

Compound 25: mp: $193.5-196.2{ }^{\circ} \mathrm{C}$; TLC (petroleum ether:EtOAc, $2: 1 \mathrm{v} / \mathrm{v}$ ): $R_{f}=0.58$; $[\alpha]_{\mathrm{D}}^{25}+18.9\left(c 1.13, \mathrm{CHCl}_{3}\right) ;{ }^{1} \mathrm{H} \mathrm{NMR}\left(600 \mathrm{MHz}, \mathrm{CDCl}_{3}, 50{ }^{\circ} \mathrm{C}\right) \delta 7.37(\mathrm{~d}, J=6.0 \mathrm{~Hz}, 1 \mathrm{H})$, $6.22(\mathrm{~d}, J=6.0 \mathrm{~Hz}, 1 \mathrm{H}), 4.29(\mathrm{~d}, J=12.0 \mathrm{~Hz}, 1 \mathrm{H}), 4.27(\mathrm{~d}, J=7.8 \mathrm{~Hz}, 1 \mathrm{H}), 4.18$ (br s, 1H), $3.73(\mathrm{~s}, 1 \mathrm{H}), 3.63(\mathrm{~d}, J=12.0 \mathrm{~Hz}, 1 \mathrm{H}), 2.94-2.70(\mathrm{~m}, 2 \mathrm{H}), 2.65-2.54(\mathrm{~m}, 2 \mathrm{H}), 2.49$ $-2.40(\mathrm{~m}, 1 \mathrm{H}), 2.00-1.81(\mathrm{~m}, 3 \mathrm{H}), 1.81-1.68(\mathrm{~m}, 2 \mathrm{H}), 1.63-1.49(\mathrm{~m}, 1 \mathrm{H}), 1.29(\mathrm{~s}, 3 \mathrm{H})$; ${ }^{13} \mathrm{C}$ NMR (151 MHz, $\left.\mathrm{CDCl}_{3}, 50{ }^{\circ} \mathrm{C}\right) \delta 205.4,195.0,170.0,154.4,135.4,133.6,66.4,60.0$, $51.7,50.4,48.3,44.4,40.0,35.3,35.0,31.6,26.2,25.1,23.2 ; \mathrm{IR}(\mathrm{KBr}): v=2960,2925$, 1708, 1670, 1637, 1260, 806, $755 \mathrm{~cm}^{-1}$; HRMS (DART, $\mathrm{m} / z$ ): calcd for $\mathrm{C}_{19} \mathrm{H}_{22} \mathrm{Cl}_{3} \mathrm{O}_{2}$ $[\mathrm{M}+\mathrm{H}]^{+}$387.0680, found 387.0675.

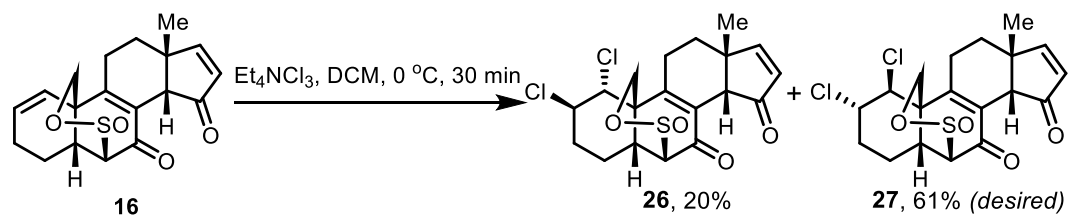

To a solution of $16(62.0 \mathrm{mg}, 0.18 \mathrm{mmol}, 1.0$ equiv) in $2.0 \mathrm{~mL}$ DCM was added dropwise a solution of $\mathrm{Et}_{4} \mathrm{NCl}_{3}\left(85.4 \mathrm{mg}, 0.36 \mathrm{mmol}, 2.5\right.$ equiv) in $0.4 \mathrm{~mL} \mathrm{DCM}$ at $0{ }^{\circ} \mathrm{C}$. The solution was stirred at $0{ }^{\circ} \mathrm{C}$ for 30 min before it was quenched with sat. aq. $\mathrm{NaHCO}_{3}(5 \mathrm{~mL})$. The resultant mixture was extracted with DCM $(3 \times 20 \mathrm{~mL})$, and the combined organic layers were washed with brine $(20 \mathrm{~mL})$, dried over anhydrous $\mathrm{Na}_{2} \mathrm{SO}_{4}$ and filtered. The volatiles were removed under vacuum, and the residue was purified by flash column chromatography with petroleum ether/EtOAc $(4: 1 \rightarrow 3: 1 \rightarrow 2: 1)$ to give compound $26(15.0$ $\mathrm{mg}, 20 \%)$ and compound $27(45.0 \mathrm{mg}, 61 \%)$ as yellow foam.

Compound 26: TLC (petroleum ether:EtOAc, 3:1 v/v): $R_{f}=0.45 ;[\alpha]_{\mathrm{D}}^{25}+135.8(c 0.60$, $\left.\mathrm{CHCl}_{3}\right) ;{ }^{1} \mathrm{H} \mathrm{NMR}\left(400 \mathrm{MHz}, \mathrm{CDCl}_{3}\right) \delta 7.45(\mathrm{~d}, J=5.6 \mathrm{~Hz}, 1 \mathrm{H}), 6.28(\mathrm{~d}, J=5.6 \mathrm{~Hz}, 1 \mathrm{H})$, 
$4.43(\mathrm{~d}, J=12.0 \mathrm{~Hz}, 1 \mathrm{H}), 4.08(\mathrm{~d}, J=12.0 \mathrm{~Hz}, 1 \mathrm{H}), 4.00(\mathrm{~d}, J=12.0 \mathrm{~Hz}, 1 \mathrm{H}), 3.82(\mathrm{td}, J$ $=11.6,4.4 \mathrm{~Hz}, 1 \mathrm{H}), 3.77(\mathrm{~s}, 1 \mathrm{H}), 3.40(\mathrm{~d}, J=1.6 \mathrm{~Hz}, 1 \mathrm{H}), 3.29$ (dt, $J=17.6,4.0 \mathrm{~Hz}, 1 \mathrm{H})$, 3.01 (ddd, $J=12.3,3.9,2.2 \mathrm{~Hz}, 1 \mathrm{H}), 2.56-2.44(\mathrm{~m}, 1 \mathrm{H}), 2.06-1.78(\mathrm{~m}, 4 \mathrm{H}), 1.62-1.58$ (m, 1H), $1.54-1.45(\mathrm{~m}, 1 \mathrm{H}), 1.33(\mathrm{~s}, 3 \mathrm{H}) ;{ }^{13} \mathrm{C} \mathrm{NMR}\left(126 \mathrm{MHz}, \mathrm{CDCl}_{3}\right) \delta 206.0,186.8$, 170.8, 152.7, 136.5, 133.6, 69.3, 68.9, 59.8, 59.3, 50.9, 46.4, 44.9, 37.4, 35.5, 34.7, 26.8, 26.5, 23.7; IR (KBr): $v=2960,2926,1711,1666,1454,1136,804,733 \mathrm{~cm}^{-1}$; HRMS (ESI, $m / z$ ): calcd for $\mathrm{C}_{19} \mathrm{H}_{21} \mathrm{Cl}_{2} \mathrm{O}_{4} \mathrm{~S}[\mathrm{M}+\mathrm{H}]^{+} 415.0532$, found 415.0532 .

Compound 27: TLC (petroleum ether:EtOAc, 3:1 v/v): $R_{f}=0.40$; $[\alpha]_{\mathrm{D}}^{25}+204.9(c 0.42$, $\left.\mathrm{CHCl}_{3}\right) ;{ }^{1} \mathrm{H} \mathrm{NMR}\left(400 \mathrm{MHz}, \mathrm{CDCl}_{3}\right) \delta 7.38(\mathrm{~d}, J=5.6 \mathrm{~Hz}, 1 \mathrm{H}), 6.25(\mathrm{~d}, J=5.6 \mathrm{~Hz}, 1 \mathrm{H})$, $4.88(\mathrm{~d}, J=11.6 \mathrm{~Hz}, 1 \mathrm{H}), 4.48(\mathrm{~d}, J=2.8 \mathrm{~Hz}, 1 \mathrm{H}), 4.38(\mathrm{dd}, J=6.0,2.8 \mathrm{~Hz}, 1 \mathrm{H}), 3.78$ (s, 1H), $3.45-3.36$ (m, 2H), 3.39 (s, 1H), $2.58-2.48$ (m, 1H), 2.43 (ddd, $J=17.6,13.2,4.0$ Hz, 1H), 2.20 (dt, $J=17.6,3.2 \mathrm{~Hz}, 1 \mathrm{H}), 2.04$ (td, $J=13.2,3.2 \mathrm{~Hz}, 1 \mathrm{H}), 1.98-1.86$ (m, $2 \mathrm{H}), 1.37$ (td, $J=13.2,3.6 \mathrm{~Hz}, 1 \mathrm{H}), 1.38-1.29(\mathrm{~m}, 1 \mathrm{H}), 1.34(\mathrm{~s}, 3 \mathrm{H}) ;{ }^{13} \mathrm{C} \mathrm{NMR}(126 \mathrm{MHz}$, $\left.\mathrm{CDCl}_{3}\right) \delta 204.8,187.3,170.6,155.0,134.8,134.6,71.0,59.9,59.9,58.7,49.8,46.2,43.8$, 34.4, 28.6, 27.6, 27.4, 26.0, 20.9; IR (KBr): $v=2960,2926,1709,1660,1262,1134,804$, $734 \mathrm{~cm}^{-1}$; HRMS (ESI, $m / z$ ): calcd for $\mathrm{C}_{19} \mathrm{H}_{21} \mathrm{Cl}_{2} \mathrm{O}_{4} \mathrm{~S}[\mathrm{M}+\mathrm{H}]^{+}$415.0532, found 415.0531 .

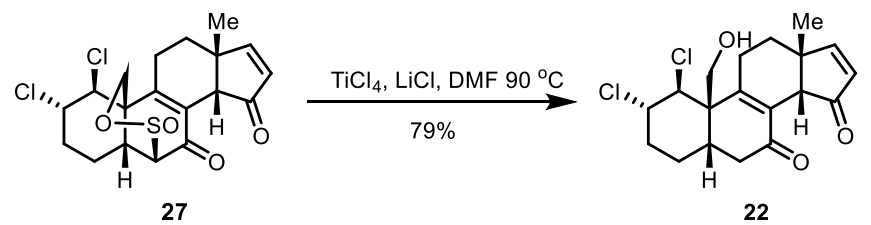

To a solution of $27(5.0 \mathrm{mg}, 0.012 \mathrm{mmol}, 1.0$ equiv) in $0.5 \mathrm{~mL}$ DMF at room temperature was added $\mathrm{LiCl}\left(2.5 \mathrm{mg}, 0.06 \mathrm{mmol}, 5.0\right.$ equiv) and $\mathrm{TiCl}_{4}(1.0 \mathrm{M}$ in $\mathrm{DCM}, 60.0 \mu \mathrm{L}, 0.06$ mmol, 5.0 equiv) under an argon atmosphere. The mixture was heated at $90{ }^{\circ} \mathrm{C}$ for $2 \mathrm{~h}$, and then quenched with sat. aq. $\mathrm{NaHCO}_{3}(5 \mathrm{~mL})$. The resultant mixture was extracted with DCM $(3 \times 5 \mathrm{~mL})$ and the combined organic layers were washed with brine $(5 \mathrm{~mL})$, dried over anhydrous $\mathrm{Na}_{2} \mathrm{SO}_{4}$ and filtered. The volatiles were removed under vacuum. The residue was subjected to flash column chromatography for purification using petroleum 
ether/acetone (4:1) as eluent to give $22(3.2 \mathrm{mg}, 79 \%)$ as colorless oil.

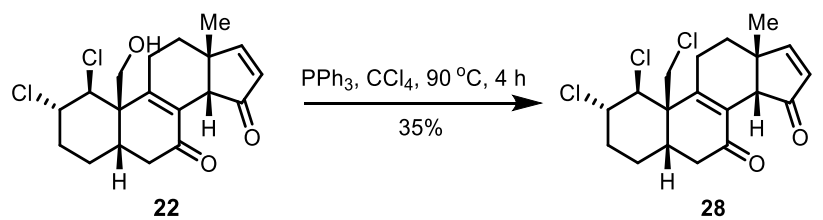

To a solution of $22(50.0 \mathrm{mg}, 0.14 \mathrm{mmol}, 1.0$ equiv $)$ in $2.5 \mathrm{~mL} \mathrm{CCl}_{4}$ was added $\mathrm{PPh}_{3}(107.0$ $\mathrm{mg}, 0.42 \mathrm{mmol}, 3.0$ equiv) at room temperature, and the solution was heated at $90{ }^{\circ} \mathrm{C}$ for $14 \mathrm{~h}$. The volatiles were removed under vacuum, and the residue was purified by flash column chromatography with petroleum ether/EtOAc (6:1) to give compound 28 (19.0 $\mathrm{mg}, 35 \%$ ) as a yellow solid.

Compound 28: mp: $156.5-157.1{ }^{\circ} \mathrm{C}$; TLC (petroleum ether:EtOAc, $2: 1 \mathrm{v} / \mathrm{v}$ ): $R_{f}=0.40$; $[\alpha]_{\mathrm{D}}^{25}+89.7\left(c\right.$ 1.36, $\left.\mathrm{CHCl}_{3}\right) ;{ }^{1} \mathrm{H} \mathrm{NMR}\left(600 \mathrm{MHz}, \mathrm{CDCl}_{3}, 50{ }^{\circ} \mathrm{C}\right) \delta 7.33(\mathrm{~d}, J=6.0 \mathrm{~Hz}$, $1 \mathrm{H}), 6.25(\mathrm{~d}, J=6.0 \mathrm{~Hz}, 1 \mathrm{H}), 4.44(\mathrm{~d}, J=8.4 \mathrm{~Hz}, 1 \mathrm{H}), 4.23(\mathrm{~d}, J=12.0 \mathrm{~Hz}, 1 \mathrm{H}), 4.15-$ $4.07(\mathrm{~m}, 1 \mathrm{H}), 3.87(\mathrm{~d}, J=12.0 \mathrm{~Hz}, 1 \mathrm{H}), 3.79(\mathrm{~s}, 1 \mathrm{H}), 2.82-2.70(\mathrm{~m}, 2 \mathrm{H}), 2.59$ (dd, $J=$ 16.2, 3.6 Hz, 1H), $2.41-2.34(\mathrm{~m}, 2 \mathrm{H}), 2.08-1.94(\mathrm{~m}, 2 \mathrm{H}), 1.83(\mathrm{dt}, J=13.8,3.6 \mathrm{~Hz}$, 1H), $1.81-1.77(\mathrm{~m}, 1 \mathrm{H}), 1.75-1.69(\mathrm{~m}, 1 \mathrm{H}), 1.52(\mathrm{td}, J=13.2,3.0 \mathrm{~Hz}, 1 \mathrm{H}), 1.30(\mathrm{~s}$, $3 \mathrm{H}) ;{ }^{13} \mathrm{C} \mathrm{NMR}\left(151 \mathrm{MHz}, \mathrm{CDCl}_{3}, 50{ }^{\circ} \mathrm{C}\right) \delta 205.7,194.4,170.2,158.7,135.3,134.2,67.6$, $60.5,50.9,49.6,46.8,45.2,38.8,35.8,35.3,30.2,27.2,26.5,25.3$; IR (KBr): $v=2957$, 2925, 1704, 1668, 1262, 1241, 800, $734 \mathrm{~cm}^{-1}$; HRMS (ESI, $\mathrm{m} / z$ ): calcd for $\mathrm{C}_{19} \mathrm{H}_{22} \mathrm{Cl}_{3} \mathrm{O}_{2}$ $[\mathrm{M}+\mathrm{H}]^{+}$387.0680, found 387.0676.

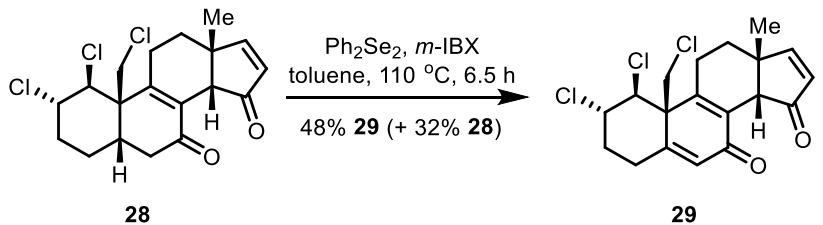

A suspension of $\mathrm{Ph}_{2} \mathrm{Se}_{2}(1.9 \mathrm{mg}, 0.006 \mathrm{mmol}, 0.2$ equiv) and $m$-IBX (13.2 mg, $0.05 \mathrm{mmol}$, 1.5 equiv) in $1.0 \mathrm{~mL}$ toluene was refluxed at $135{ }^{\circ} \mathrm{C}$ for $1 \mathrm{~h}$ until the color turned from yellow to white. Then a solution of $28(12.0 \mathrm{mg}, 0.03 \mathrm{mmol}, 1.0$ equiv) in $0.5 \mathrm{~mL}$ toluene 
was added to the above suspension and the mixture was heated at $110^{\circ} \mathrm{C}$ for $6.5 \mathrm{~h}$. After cooling to room temperature, the reaction mixture was quenched with sat. aq. $\mathrm{NaHCO}_{3}(5$ $\mathrm{mL})$. The resultant mixture was extracted with $\mathrm{DCM}(3 \times 10 \mathrm{~mL})$, and the combined organic layers were washed with brine $(10 \mathrm{~mL})$, dried over anhydrous $\mathrm{Na}_{2} \mathrm{SO}_{4}$ and filtered. The volatiles were removed under vacuum, and the residue was purified by flash column chromatography with petroleum ether/EtOAc $(8: 1 \rightarrow 7: 1)$ to give compound $28(3.8 \mathrm{mg}$, $32 \%)$ and compound $29(5.7 \mathrm{mg}, 48 \%)$ as yellow solids.

Compound 29: mp: $183.4-184.8^{\circ} \mathrm{C}$; TLC (petroleum ether:EtOAc, $2: 1 \mathrm{v} / \mathrm{v}$ ): $R_{f}=0.35$; $[\alpha]_{\mathrm{D}}^{25}+181.0\left(c\right.$ 0.32, $\left.\mathrm{CHCl}_{3}\right) ;{ }^{1} \mathrm{H} \mathrm{NMR}\left(400 \mathrm{MHz}, \mathrm{CDCl}_{3}\right) \delta 7.39(\mathrm{~d}, J=5.6 \mathrm{~Hz}, 1 \mathrm{H})$, $6.48(\mathrm{~s}, 1 \mathrm{H}), 6.33(\mathrm{~d}, J=5.6 \mathrm{~Hz}, 1 \mathrm{H}), 4.36(\mathrm{~d}, J=12.0 \mathrm{~Hz}, 1 \mathrm{H}), 4.10(\mathrm{ddd}, J=12.4,10.8$, $5.2 \mathrm{~Hz}, 1 \mathrm{H}), 3.91(\mathrm{~s}, 1 \mathrm{H}), 3.87(\mathrm{~d}, J=12.0 \mathrm{~Hz}, 1 \mathrm{H}), 3.83(\mathrm{~d}, J=10.4 \mathrm{~Hz}, 1 \mathrm{H}), 2.66-2.52$ (m, 4H), 1.99 (ddd, $J=16.4,13.2,3.2 \mathrm{~Hz}, 1 \mathrm{H}), 1.96-1.83(\mathrm{~m}, 2 \mathrm{H}), 1.46(\mathrm{td}, J=13.2$, $2.8 \mathrm{~Hz}, 1 \mathrm{H}), 1.33$ (s, 3H); ${ }^{13} \mathrm{C}$ NMR $\left(101 \mathrm{MHz}, \mathrm{CDCl}_{3}\right) \delta$ 206.4, 182.6, 170.5, 157.0, 155.7, 137.0, 134.6, 129.9, 71.6, 61.5, 54.0, 50.3, 45.5, 42.5, 37.2, 35.3, 31.5, 27.3, 26.1; IR (KBr): $v=3048,2958,2925,1708,1667,1634,1388,1284,736 \mathrm{~cm}^{-1}$; HRMS (ESI, $m / z$ ): calcd for $\mathrm{C}_{19} \mathrm{H}_{20} \mathrm{Cl}_{3} \mathrm{O}_{2}[\mathrm{M}+\mathrm{H}]^{+}$385.0523, found 385.0521 .
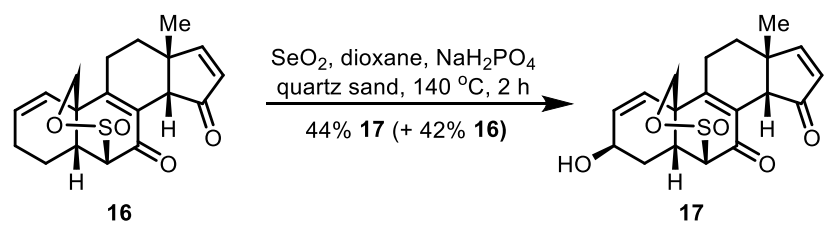

A sealed tube was charged with olefin $16(20.0 \mathrm{mg}, 0.06 \mathrm{mmol}, 1.0$ equiv), sodium hydrogen phosphate $(9.0 \mathrm{mg}, 0.06 \mathrm{mmol}, 1.0$ equiv), finely grounded selenium dioxide (6.5 $\mathrm{mg}, 0.06 \mathrm{mmol}, 1.0$ equiv), quartz sand $(26.0 \mathrm{mg})$ and $1.2 \mathrm{~mL}$ anhydrous dioxane. After stirring at $140{ }^{\circ} \mathrm{C}$ for $1 \mathrm{~h}$, selenium dioxide $(6.5 \mathrm{mg}, 0.06 \mathrm{mmol}, 1.0$ equiv) and quartz sand (26.0 mg) were added. The solution was stirred at $140{ }^{\circ} \mathrm{C}$ for an additional $1 \mathrm{~h}$ and then quenched with $\mathrm{H}_{2} \mathrm{O}(1 \mathrm{~mL})$. After stirred for about $20 \mathrm{~min}$ at room temperature, the reaction mixture was filtered through a short plug of silica gel and washed with DCM $(5 \mathrm{~mL})$ and 
$\mathrm{H}_{2} \mathrm{O}(5 \mathrm{~mL})$. The reaction mixture was extracted with DCM $(3 \times 10 \mathrm{~mL})$, and the combined organic layers were washed with brine $(10 \mathrm{~mL})$, dried over anhydrous $\mathrm{Na}_{2} \mathrm{SO}_{4}$ and filtered. The volatiles were removed under vacuum, and the residue was purified by flash column chromatography with petroleum ether/acetone $(8: 1 \rightarrow 7: 1 \rightarrow 2: 1)$ to give compound $16(8.4$ $\mathrm{mg}, 42 \%)$ as a yellow solid, and compound $17(9.2 \mathrm{mg}, 44 \%)$ as yellow wax.

Compound 17: TLC (petroleum ether:acetone, 3:2 v/v): $R_{f}=0.18 ;[\alpha]_{\mathrm{D}}^{25}+278.8(c 0.83$, $\left.\mathrm{CHCl}_{3}\right) ;{ }^{1} \mathrm{H} \mathrm{NMR}\left(400 \mathrm{MHz}, \mathrm{CD}_{3} \mathrm{OD}\right) \delta 7.58(\mathrm{~d}, J=5.6 \mathrm{~Hz}, 1 \mathrm{H}), 6.20(\mathrm{~d}, J=5.6 \mathrm{~Hz}, 1 \mathrm{H})$, 6.06 (ddd, $J=10.0,4.4,1.2 \mathrm{~Hz}, 1 \mathrm{H}), 5.75$ (d, $J=10.0 \mathrm{~Hz}, 1 \mathrm{H}), 4.29$ (d, $J=11.6 \mathrm{~Hz}, 1 \mathrm{H})$, $4.05(\mathrm{t}, J=4.4 \mathrm{~Hz}, 1 \mathrm{H}), 3.82(\mathrm{~d}, J=11.6 \mathrm{~Hz}, 1 \mathrm{H}), 3.61(\mathrm{~s}, 1 \mathrm{H}), 3.46(\mathrm{~d}, J=2.0 \mathrm{~Hz}, 1 \mathrm{H})$, $3.36(\mathrm{dt}, J=13.3,2.4 \mathrm{~Hz}, 1 \mathrm{H}), 2.36$ (dt, $J=17.6,3.6 \mathrm{~Hz}, 1 \mathrm{H}), 1.93(\mathrm{dt}, J=13.2,4.0 \mathrm{~Hz}$, 1H), $1.88-1.70(\mathrm{~m}, 2 \mathrm{H}), 1.58(\mathrm{ddt}, J=14.2,3.0,1.5 \mathrm{~Hz}, 1 \mathrm{H}), 1.42(\mathrm{td}, J=13.2,3.6 \mathrm{~Hz}$, 1H), $1.31(\mathrm{~s}, 3 \mathrm{H}) ;{ }^{13} \mathrm{C} \mathrm{NMR}\left(126 \mathrm{MHz}, \mathrm{CD}_{3} \mathrm{OD}\right) \delta 208.5,189.2,173.8,158.2,135.0,134.1$, 134.0, 128.3, 72.4, 63.1, 62.3, 51.1, 47.0, 42.7, 34.8, 33.7, 28.6, 27.6, 25.3; IR (KBr): $v=$ $3459,3019,2926,1705,1659,1141,935,753 \mathrm{~cm}^{-1}$; HRMS (ESI, $\mathrm{m} / z$ ): calcd for $\mathrm{C}_{19} \mathrm{H}_{21} \mathrm{O}_{5} \mathrm{~S}$ $[\mathrm{M}+\mathrm{H}]^{+}$361.1104, found 361.1104.
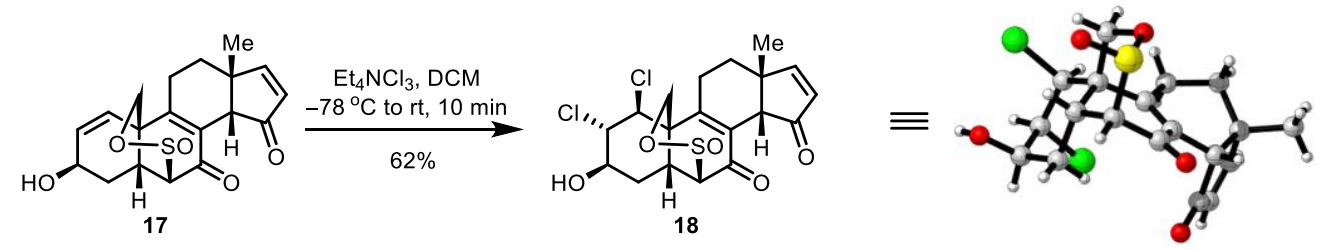

To a stirred solution of $17\left(93.0 \mathrm{mg}, 0.26 \mathrm{mmol}, 1.0\right.$ equiv) in $4.7 \mathrm{~mL} \mathrm{DCM}$ at $-78{ }^{\circ} \mathrm{C}$ was added a solution of $\mathrm{Et}_{4} \mathrm{NCl}_{3}(186.0 \mathrm{mg}, 0.84 \mathrm{mmol}, 3.0$ equiv) in $4.3 \mathrm{~mL}$ DCM slowly. After the reaction mixture was stirred at $-78{ }^{\circ} \mathrm{C}$ for about $10 \mathrm{~min}$, the cooling bath was removed and the reaction mixture was allowed to slowly warm to room temperature for 10 min. Then a solution of $\mathrm{Et}_{4} \mathrm{NCl}_{3}(19.0 \mathrm{mg}, 0.084 \mathrm{mmol}, 0.3$ equiv) in $0.5 \mathrm{~mL} \mathrm{DCM}$ was added at room temperature, and the reaction mixture was stirred for $1 \mathrm{~min}$ before it was quenched with $\mathrm{H}_{2} \mathrm{O}(5 \mathrm{~mL})$. The reaction mixture was extracted with $\mathrm{DCM}(3 \times 30 \mathrm{~mL})$, 
and the combined organic layers were washed with brine $(30 \mathrm{~mL})$, dried over anhydrous $\mathrm{Na}_{2} \mathrm{SO}_{4}$ and filtered. The volatiles were removed under vacuum, and the residue was purified by flash column chromatography with petroleum ether/acetone $(7: 1 \rightarrow 6: 1 \rightarrow 5: 1)$ to give compound $18(69.0 \mathrm{mg}, 62 \%)$ as a yellow solid.

Compound 18: $\mathrm{mp}: 150.2-153.1{ }^{\circ} \mathrm{C}$; TLC (petroleum ether:acetone, $3: 2 \mathrm{v} / \mathrm{v}$ ): $R_{f}=0.45$; $[\alpha]_{\mathrm{D}}^{25}+167.5\left(c 0.77, \mathrm{CHCl}_{3}\right) ;{ }^{1} \mathrm{H} \mathrm{NMR}\left(400 \mathrm{MHz}, \mathrm{CD}_{3} \mathrm{OD}\right) \delta 7.61(\mathrm{~d}, J=5.6 \mathrm{~Hz}, 1 \mathrm{H})$, $6.22(\mathrm{~d}, J=5.6 \mathrm{~Hz}, 1 \mathrm{H}), 4.95(\mathrm{~d}, J=11.6 \mathrm{~Hz}, 1 \mathrm{H}), 4.79($ br s, 1H), $4.46(\mathrm{dd}, J=4.4,2.0$ $\mathrm{Hz}, 1 \mathrm{H}), 4.04(\mathrm{dd}, J=5.6,2.8 \mathrm{~Hz}, 1 \mathrm{H}), 3.82(\mathrm{dt}, J=13.2,3.2 \mathrm{~Hz}, 1 \mathrm{H}), 3.71(\mathrm{~s}, 1 \mathrm{H}), 3.65$ $(\mathrm{d}, J=11.6 \mathrm{~Hz}, 1 \mathrm{H}), 3.48(\mathrm{~d}, J=2.0 \mathrm{~Hz}, 1 \mathrm{H}), 2.47(\mathrm{dt}, J=17.6,3.2 \mathrm{~Hz}, 1 \mathrm{H}), 2.35-2.15$ (m, 2H), $2.02(\mathrm{dt}, J=13.2,3.6 \mathrm{~Hz}, 1 \mathrm{H}), 1.52-1.43(\mathrm{~m}, 1 \mathrm{H}), 1.39-1.27(\mathrm{~m}, 2 \mathrm{H}), 1.33(\mathrm{~s}$, $3 \mathrm{H}) ;{ }^{13} \mathrm{C}$ NMR (101 MHz, CD $\left.3 \mathrm{OD}\right) \delta 206.4,187.5,172.4,156.7,134.5,133.3,70.3,69.0$, $60.0,59.0,56.9,49.6,46.2,43.4,33.9,27.3,26.2,25.0,22.8$; IR (KBr): $v=3448,2960$, 2923, 1698, 1685, 1305, 922, $784 \mathrm{~cm}^{-1}$; HRMS (ESI, $m / z$ ): calcd for $\mathrm{C}_{19} \mathrm{H}_{21} \mathrm{Cl}_{2} \mathrm{O}_{5} \mathrm{~S}[\mathrm{M}+\mathrm{H}]^{+}$ 431.0481 , found 431.0482 .
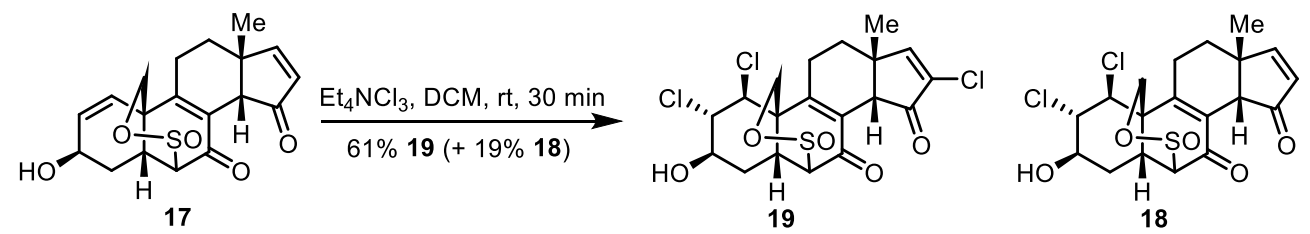

To a stirred solution of $\mathrm{Et}_{4} \mathrm{NCl}_{3}(52.0 \mathrm{mg}, 0.22 \mathrm{mmol}, 8.0$ equiv) in $1.0 \mathrm{~mL} \mathrm{DCM}$ at room temperature was added a solution of $17(10.0 \mathrm{mg}, 0.03 \mathrm{mmol}, 1.0$ equiv) in $1.0 \mathrm{~mL} \mathrm{DCM}$ slowly. The reaction mixture was stirred for 30 min before it was quenched with $\mathrm{Et}_{3} \mathrm{~N}$ (2 $\mathrm{mL})$ and $\mathrm{H}_{2} \mathrm{O}(2 \mathrm{~mL})$. The reaction mixture was extracted with $\mathrm{DCM}(3 \times 5 \mathrm{~mL})$, and the combined organic layers were washed with brine $(5 \mathrm{~mL})$, dried over anhydrous $\mathrm{Na}_{2} \mathrm{SO}_{4}$ and filtered. The volatiles were removed under vacuum, and the residue was purified by flash column chromatography with petroleum ether/acetone $(7: 1 \rightarrow 6: 1 \rightarrow 5: 1)$ to give compound $19(8.0 \mathrm{mg}, 61 \%)$ and compound $18(2.3 \mathrm{mg}, 19 \%)$ as white solids.

Compound 19: $\mathrm{mp}: 252.2-253.5{ }^{\circ} \mathrm{C}$; TLC (petroleum ether:acetone, $3: 2 \mathrm{v} / \mathrm{v}$ ): $R_{f}=0.50$; 
$[\alpha]_{\mathrm{D}}^{25}+166.2\left(c 0.38, \mathrm{CHCl}_{3}\right) ;{ }^{1} \mathrm{H} \mathrm{NMR}\left(400 \mathrm{MHz}, \mathrm{CDCl}_{3}\right) \delta 7.31(\mathrm{~s}, 1 \mathrm{H}), 5.00(\mathrm{~d}, J=11.7$ Hz, 1H), 4.53 (br s, 1H), 4.44 (q, $J=2.2 \mathrm{~Hz}, 1 \mathrm{H}$ ), 4.16 (br s, 1H), 3.94 (s, 1H), 3.82 (ddd, $J=13.6,4.0,2.8 \mathrm{~Hz}, 1 \mathrm{H}), 3.44$ (d, $J=11.7 \mathrm{~Hz}, 1 \mathrm{H}), 3.41$ (d, $J=2.3 \mathrm{~Hz}, 1 \mathrm{H}), 2.47-2.32$ (m, 1H), $2.28-2.16(\mathrm{~m}, 2 \mathrm{H}), 1.99$ (dt, $J=13.4,3.5 \mathrm{~Hz}, 1 \mathrm{H}), 1.42$ (td, $J=13.1,3.6 \mathrm{~Hz}$, 1H), 1.39 (s, 3H); $\left.{ }^{13} \mathrm{C} \mathrm{NMR} \mathrm{(101} \mathrm{MHz,} \mathrm{CDCl}_{3}\right) \delta$ 196.4, 186.7, 163.0, 155.0, 136.2, 134.6, 70.6, 70.0, 59.7, 59.6, 57.6, 49.1, 43.9, 43.8, 34.4, 28.0, 27.6, 25.8, 23.2; IR (KBr): $v=$ 2921, 1713, 1667, 1650, 922, $707 \mathrm{~cm}^{-1}$; HRMS (ESI, $\mathrm{m} / z$ ): calcd for $\mathrm{C}_{19} \mathrm{H}_{19} \mathrm{Cl}_{3} \mathrm{O}_{5} \mathrm{~S}[\mathrm{M}+\mathrm{H}]^{+}$ 465.0092, found 465.0092 .

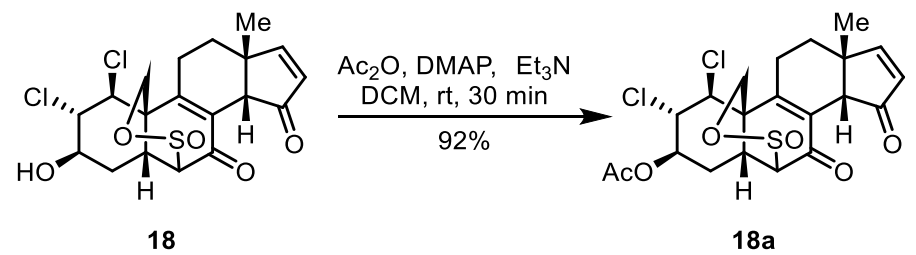

To a solution of 18 (145.0 $\mathrm{mg}, 0.33 \mathrm{mmol}, 1.0$ equiv) in $16.0 \mathrm{~mL}$ DCM was added $\mathrm{Et}_{3} \mathrm{~N}$ (1.37 mL, $0.99 \mathrm{mmol}, 3.0$ equiv), DMAP ( $8.0 \mathrm{mg}, 0.07 \mathrm{mmol}, 0.2$ equiv), and $\mathrm{Ac}_{2} \mathrm{O}$ (0.65 $\mathrm{mL}, 0.66 \mathrm{mmol}, 2.0$ equiv) at room temperature. After stirring for $30 \mathrm{~min}$, the volatiles were concentrated under vacuum. The residue was subjected to flash column chromatography for purification using petroleum ether/acetone (4:1) as eluent to give 18a (143.8 $\mathrm{mg}, 92 \%)$ as a white solid.

Compound 18a: mp: $226.4-227.5^{\circ} \mathrm{C}$; TLC (petroleum ether:actone, $2: 1 \mathrm{v} / \mathrm{v}$ ): $R_{f}=0.62$; $[\alpha]_{\mathrm{D}}^{25}+138.1\left(c 1.80, \mathrm{CHCl}_{3}\right) ;{ }^{1} \mathrm{H} \mathrm{NMR}\left(400 \mathrm{MHz}, \mathrm{CDCl}_{3}\right) \delta 7.39(\mathrm{~d}, J=5.6 \mathrm{~Hz}, 1 \mathrm{H}), 6.24$ (d, $J=5.6 \mathrm{~Hz}, 1 \mathrm{H}), 4.98$ (d, $J=11.6 \mathrm{~Hz}, 1 \mathrm{H}), 4.96$ (br s, 1H), 4.58 (br s, 1H), 4.51 (br s, 1H), 3.79 (s, 1H), 3.78 (d, $J=11.6 \mathrm{~Hz}, 1 \mathrm{H}), 3.43$ (d, $J=13.0 \mathrm{~Hz}, 1 \mathrm{H}), 3.41$ (br s, 1H), 2.39 - 2.27 (m, 2H), 2.19 (dt, $J=18.3 \mathrm{~Hz}, 2.8 \mathrm{~Hz}, 1 \mathrm{H}), 2.10$ (s, 3H), 1.95 (dt, $J=13.0 \mathrm{~Hz}$, $2.8 \mathrm{~Hz}, 1 \mathrm{H}), 1.56-1.48(\mathrm{~m}, 1 \mathrm{H}), 1.38(\mathrm{td}, J=13.0,3.6 \mathrm{~Hz}, 1 \mathrm{H}), 1.35(\mathrm{~s}, 3 \mathrm{H}) ;{ }^{13} \mathrm{C} \mathrm{NMR}$ $\left(126 \mathrm{MHz}, \mathrm{CDCl}_{3}\right) \delta 204.8,186.9,170.7,170.2,154.4,135.4,134.6,70.4,70.1,59.5,57.2$, 55.6, 49.8, 46.3, 43.4, 34.4, 27.6, 25.8, 25.5, 24.1, 21.3; IR (KBr): $v=2960,1744,1709$, 1659, 1237, 1135, 808, $745 \mathrm{~cm}^{-1}$; HRMS (ESI, $\mathrm{m} / \mathrm{z}$ ): calcd for $\mathrm{C}_{21} \mathrm{H}_{23} \mathrm{Cl}_{2} \mathrm{O}_{6} \mathrm{~S}[\mathrm{M}+\mathrm{H}]^{+}$ 
473.0587, found 473.0577 .

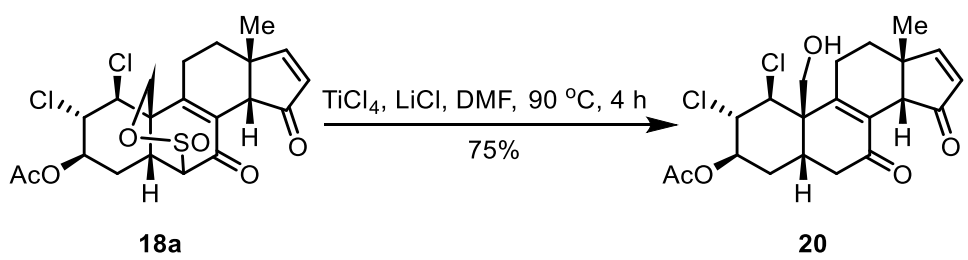

To a solution of 18a ( $36.0 \mathrm{mg}, 0.08 \mathrm{mmol}, 1.0$ equiv) in $3.0 \mathrm{~mL}$ DMF at room temperature was added $\mathrm{LiCl}\left(16.0 \mathrm{mg}, 0.38 \mathrm{mmol}, 5.0\right.$ equiv) and $\mathrm{TiCl}_{4}(1.0 \mathrm{M}$ in DCM, $0.38 \mathrm{~mL}, 0.38$ mmol, 5.0 equiv) under an argon atmosphere. The mixture was heated at $90^{\circ} \mathrm{C}$ for $4 \mathrm{~h}$, and then quenched with sat. aq. $\mathrm{NaHCO}_{3}(2 \mathrm{~mL})$. The resultant mixture was extracted with DCM $(3 \times 5 \mathrm{~mL})$ and the combined organic layers were washed with brine $(5 \mathrm{~mL})$, dried over anhydrous $\mathrm{Na}_{2} \mathrm{SO}_{4}$ and filtered. The volatiles were removed under vacuum. The residue was subjected to flash column chromatography for purification using petroleum ether/acetone (4:1) as eluent to give $\mathbf{2 0}(24.2 \mathrm{mg}, 75 \%)$ as a white solid.

Compound 20: mp: $209.5-209.9{ }^{\circ} \mathrm{C}$; TLC (petroleum ether:actone, $2: 1 \mathrm{v} / \mathrm{v}$ ): $R_{f}=0.45$; $[\alpha]_{\mathrm{D}}^{25}+116.9\left(c 0.37, \mathrm{CHCl}_{3}\right) ;{ }^{1} \mathrm{H} \mathrm{NMR}\left(600 \mathrm{MHz}, \mathrm{CDCl}_{3}\right) \delta 7.32(\mathrm{~d}, J=5.6 \mathrm{~Hz}, 1 \mathrm{H}), 6.24$ (d, $J=5.6 \mathrm{~Hz}, 1 \mathrm{H}), 5.07$ (td, $J=7.2,4.2 \mathrm{~Hz}, 1 \mathrm{H}), 4.54$ (d, $J=7.3 \mathrm{~Hz}, 1 \mathrm{H}), 4.32$ (d, $J=$ $11.2 \mathrm{~Hz}, 1 \mathrm{H}), 4.26$ (br s, 1H), 4.02 (dd, $J=11.2,4.6 \mathrm{~Hz}, 1 \mathrm{H}), 3.77$ (s, 1H), $2.90-2.82$ (m, 1H), $2.70(\mathrm{td}, J=17.2,4.9 \mathrm{~Hz}, 1 \mathrm{H}), 2.67-2.59(\mathrm{~m}, 1 \mathrm{H}), 2.49$ (dt, $J=16.6,3.4 \mathrm{~Hz}, 1 \mathrm{H})$, $2.18-2.11(\mathrm{~m}, 1 \mathrm{H}), 2.08(\mathrm{~s}, 3 \mathrm{H}), 2.07-2.00(\mathrm{~m}, 1 \mathrm{H}), 1.89-1.83(\mathrm{~m}, 1 \mathrm{H}), 1.82(\mathrm{dt}, J=$ $13.2,3.0 \mathrm{~Hz}, 1 \mathrm{H}), 1.77(\mathrm{t}, J=6.0 \mathrm{~Hz}, 1 \mathrm{H}), 1.44(\mathrm{td}, J=13.2,3.0 \mathrm{~Hz}, 1 \mathrm{H}), 1.30(\mathrm{~s}, 3 \mathrm{H}) ;{ }^{13} \mathrm{C}$ $\operatorname{NMR}\left(151 \mathrm{MHz}, \mathrm{CDCl}_{3}, 50^{\circ} \mathrm{C}\right) \delta 205.9,194.1,170.1,169.9,160.2,134.3(2 \mathrm{C}), 71.8,64.5$, $60.6,50.8,48.7,45.5,39.2,35.4,32.0,30.2,27.2,26.4,21.1$ (the $\mathrm{C}_{19} \mathrm{CH}_{2}$ carbon was not observed); IR (KBr): $v=3417,2924,1743,1698,1661,1238,1046 \mathrm{~cm}^{-1}$; HRMS (ESI, $m / z$ ): calcd for $\mathrm{C}_{21} \mathrm{H}_{24} \mathrm{Cl}_{2} \mathrm{NaO}_{5}[\mathrm{M}+\mathrm{Na}]^{+} 449.0893$, found 449.0890 . 


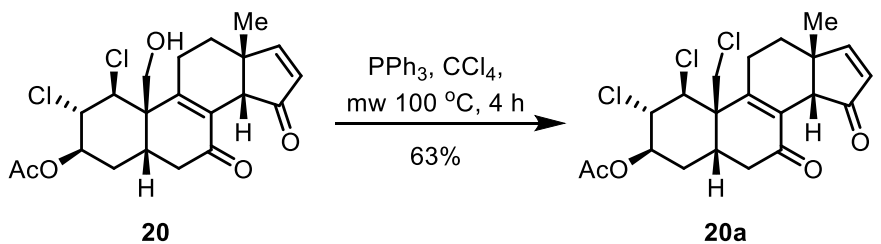

To a $2 \mathrm{~mL}$ microwave vial was added 20 (10.0 mg, $0.02 \mathrm{mmol}, 1.0$ equiv), $\mathrm{PPh}_{3}(18.0 \mathrm{mg}$, $0.07 \mathrm{mmol}, 3.0$ equiv), and $0.8 \mathrm{~mL} \mathrm{CCl}_{4}$ under an argon atmosphere. The vial was sealed and heated under microwave irradiation at $100^{\circ} \mathrm{C}$ for $4 \mathrm{~h}$. After the volatiles were removed under vacuum, the residue was subjected to flash column chromatography for purification using petroleum ether/acetone (6:1) as eluent to give 20a $(6.4 \mathrm{mg}, 63 \%)$ as a white solid.

Compound 20a: mp: $211.7-212.5{ }^{\circ} \mathrm{C}$; TLC (petroleum ether:actone, $4: 1 \mathrm{v} / \mathrm{v}$ ): $R_{f}=0.40$; $[\alpha]_{\mathrm{D}}^{25}+72.3\left(c 1.30, \mathrm{CHCl}_{3}\right) ;{ }^{1} \mathrm{H} \mathrm{NMR}\left(600 \mathrm{MHz}, \mathrm{CDCl}_{3}\right) \delta 7.33(\mathrm{~d}, J=5.6 \mathrm{~Hz}, 1 \mathrm{H}), 6.27$ $(\mathrm{d}, J=5.6 \mathrm{~Hz}, 1 \mathrm{H}), 5.17(\mathrm{ddd}, J=10.2,9.5,5.5 \mathrm{~Hz}, 1 \mathrm{H}), 4.44(\mathrm{~d}, J=10.2 \mathrm{~Hz}, 1 \mathrm{H}), 4.39$ (d, $J=12.2 \mathrm{~Hz}, 1 \mathrm{H}), 4.03(\mathrm{t}, J=10.2 \mathrm{~Hz}, 1 \mathrm{H}), 3.86(\mathrm{~d}, J=12.2 \mathrm{~Hz}, 1 \mathrm{H}), 3.79(\mathrm{~s}, 1 \mathrm{H}), 3.04$ $(\mathrm{dq}, J=13.8,4.8 \mathrm{~Hz}, 1 \mathrm{H}), 2.82(\mathrm{dd}, J=17.1,13.8 \mathrm{~Hz}, 1 \mathrm{H}), 2.65(\mathrm{dd}, J=17.1,4.8 \mathrm{~Hz}, 1 \mathrm{H})$, $2.38(\mathrm{ddd}, J=16.8,4.0,3.0 \mathrm{~Hz}, 1 \mathrm{H}), 2.09$ (s, 3H), $2.04(\mathrm{ddd}, J=13.8,5.5,3.4 \mathrm{~Hz}, 1 \mathrm{H})$, $1.96(\mathrm{ddd}, J=16.8,12.8,3.5 \mathrm{~Hz}, 1 \mathrm{H}), 1.90(\mathrm{ddd}, J=13.8,8.4,4.7 \mathrm{~Hz}, 1 \mathrm{H}), 1.84(\mathrm{dt}, J=$ 13.8, 3.0 Hz, 1H), $1.52(\mathrm{td}, J=8.4,3.0 \mathrm{~Hz}, 1 \mathrm{H}), 1.30(\mathrm{~s}, 3 \mathrm{H}) ;{ }^{13} \mathrm{C} \mathrm{NMR}\left(151 \mathrm{MHz}, \mathrm{CDCl}_{3}\right.$, $\left.50{ }^{\circ} \mathrm{C}\right) \delta 205.8,193.4,170.1,169.6,158.5,136.4,134.2,72.0,66.9,63.5,51.2,50.3,44.9$, 44.2, 38.7, 35.6, 34.7, 31.2, 27.0, 26.1, 20.9; IR (KBr): v = 2959, 2925, 1744, 1705, 1673, 1367, 1237, 1051, $796 \mathrm{~cm}^{-1}$; HRMS (ESI, m/z): calcd for $\mathrm{C}_{21} \mathrm{H}_{27} \mathrm{Cl}_{3} \mathrm{NO}_{4}\left[\mathrm{M}+\mathrm{NH}_{4}\right]^{+}$ 462.1000, found 462.0097 .

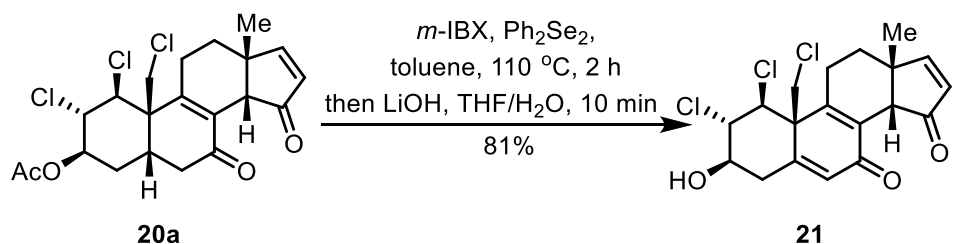

A solution of $m$-IBX (24.7 mg, $0.08 \mathrm{mmol}, 1.5$ equiv) and $\mathrm{Ph}_{2} \mathrm{Se}_{2}(7.4 \mathrm{mg}, 0.03 \mathrm{mmol}, 0.5$ equiv) in $1.5 \mathrm{~mL}$ dry toluene was stirred at $130{ }^{\circ} \mathrm{C}$ for $0.5 \mathrm{~h}$ until the solution turned from yellow to white. Then $\mathbf{2 0 a}(23.4 \mathrm{mg}, 0.05 \mathrm{mmol}, 1.0$ equiv) was added and the solution 
was stirred for another $2 \mathrm{~h}$ at $110^{\circ} \mathrm{C}$. Removal of the volatiles under vacuum afforded the crude product, and $1.5 \mathrm{~mL} \mathrm{THF}, 1.5 \mathrm{~mL} \mathrm{H} \mathrm{H}_{2} \mathrm{O}$, and $\mathrm{LiOH} \cdot \mathrm{H}_{2} \mathrm{O}(11.0 \mathrm{mg}, 0.27 \mathrm{mmol}, 5.0$ equiv) were added at room temperature. After stirred at the same temperature for $10 \mathrm{~min}$, the reaction mixture was extracted with DCM $(3 \times 3 \mathrm{~mL})$. The combined organic layers were washed with brine $(5 \mathrm{~mL})$, dried over anhydrous $\mathrm{Na}_{2} \mathrm{SO}_{4}$, filtered, and concentrated under vacuum. The residue was subjected to flash column chromatography for purification using petroleum ether/acetone (4:1) as eluent to give 21 (17.5 mg, 81\%) as colorless oil.

Compound 21: TLC (petroleum ether:actone, 2:1 v/v): $R_{f}=0.50 ;[\alpha]_{\mathrm{D}}^{25}+198.5(c 0.29$, $\left.\mathrm{CHCl}_{3}\right) ;{ }^{1} \mathrm{H} \mathrm{NMR}\left(500 \mathrm{MHz}, \mathrm{CDCl}_{3}\right) \delta 7.40(\mathrm{~d}, J=5.6 \mathrm{~Hz}, 1 \mathrm{H}), 6.54(\mathrm{~s}, 1 \mathrm{H}), 6.32(\mathrm{~d}, J=$ $5.6 \mathrm{~Hz}, 1 \mathrm{H}), 4.36(\mathrm{~d}, J=11.5 \mathrm{~Hz}, 1 \mathrm{H}), 3.98(\mathrm{dd}, J=11.5,9.5 \mathrm{~Hz}, 1 \mathrm{H}), 3.90(\mathrm{~s}, 1 \mathrm{H}), 3.89$ $(\mathrm{d}, J=11.5 \mathrm{~Hz}, 1 \mathrm{H}), 3.82(\mathrm{~d}, J=11.5 \mathrm{~Hz}, 1 \mathrm{H}), 3.74-3.65(\mathrm{~m}, 1 \mathrm{H}), 2.90(\mathrm{dd}, J=13.4,5.7$ $\mathrm{Hz}, 1 \mathrm{H}), 2.78(\mathrm{~d}, J=2.9 \mathrm{~Hz}, 1 \mathrm{H}), 2.66-2.57(\mathrm{~m}, 2 \mathrm{H}), 2.04-1.88(\mathrm{~m}, 2 \mathrm{H}), 1.47$ (td, $J=$ 15.6, 3.0 Hz, 1H), 1.33 (s, 3H); ${ }^{13} \mathrm{C}$ NMR (126 MHz, $\left.\mathrm{CDCl}_{3}\right) \delta 206.5,182.2,170.7,156.9$, $151.3,137.0,134.5,132.1,74.3,69.7,67.8,53.6,50.3,45.5,42.5,38.4,35.2,27.3,25.9$; IR (KBr): $v=3400,2923,1698,1667,1633,1086,775 \mathrm{~cm}^{-1}$; HRMS (ESI, $m / z$ ): calcd for $\mathrm{C}_{19} \mathrm{H}_{20} \mathrm{Cl}_{3} \mathrm{O}_{3}[\mathrm{M}+\mathrm{H}]^{+} 401.0473$, found 401.0471 .

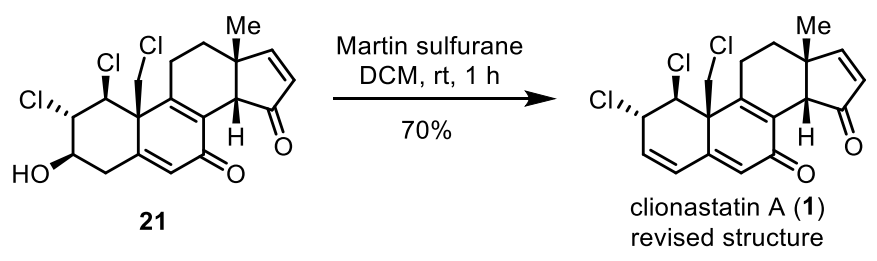

To a solution of 21 (17.5 mg, $0.04 \mathrm{mmol}, 1.0$ equiv) in $4.0 \mathrm{~mL}$ DCM was added Martin sulfurane (89.0 mg, $0.13 \mathrm{mmol}, 3.0$ equiv) under an argon atmosphere. The solution was stirred at room temperature for $1 \mathrm{~h}$, and then quenched with water $(2 \mathrm{~mL})$. The resultant mixture was extracted with DCM $(3 \times 3 \mathrm{~mL})$. The combined organic layers were washed with brine $(3 \mathrm{~mL})$, dried over anhydrous $\mathrm{Na}_{2} \mathrm{SO}_{4}$ and filtered. The volatiles were removed under vacuum. The residue was subjected to flash column chromatography for purification using petroleum ether/acetone (9:1) as eluent to give clionastatin $\mathrm{A}(\mathbf{1}, 11.7 \mathrm{mg}, 70 \%)$ as a 
white solid.

Clionastatin A: mp: $193.5-194.2^{\circ} \mathrm{C}$; TLC (petroleum ether:actone, $4: 1 \mathrm{v} / \mathrm{v}$ ): $R_{f}=0.40$; $[\alpha]_{\mathrm{D}}^{25}+93.1\left(c 0.02, \mathrm{CHCl}_{3}\right) ;{ }^{1} \mathrm{H} \mathrm{NMR}\left(600 \mathrm{MHz}, \mathrm{CDCl}_{3}\right) \delta 7.39(\mathrm{~d}, J=5.6 \mathrm{~Hz}, 1 \mathrm{H}), 6.53$ (s, 1H), $6.47(\mathrm{ddd}, J=9.9,2.2,0.9 \mathrm{~Hz}, 1 \mathrm{H}), 6.31(\mathrm{~d}, J=5.6 \mathrm{~Hz}, 1 \mathrm{H}), 6.06(\mathrm{dd}, J=9.9,2.4$ $\mathrm{Hz}, 1 \mathrm{H}), 4.71(\mathrm{dt}, J=9.0,2.4 \mathrm{~Hz}, 1 \mathrm{H}), 4.24(\mathrm{~d}, J=11.2 \mathrm{~Hz}, 1 \mathrm{H}), 4.19(\mathrm{~d}, J=9.0 \mathrm{~Hz}, 1 \mathrm{H})$, 3.99 (s, 1H), 3.76 (d, $J=11.2 \mathrm{~Hz}, 1 \mathrm{H}), 2.69$ (dt, $J=17.1,3.2 \mathrm{~Hz}, 1 \mathrm{H}), 2.08$ (ddd, $J=17.1$, 13.3, $3.5 \mathrm{~Hz}, 1 \mathrm{H}), 1.92(\mathrm{dt}, J=13.2,3.2 \mathrm{~Hz}, 1 \mathrm{H}), 1.50(\mathrm{td}, J=13.3,3.2 \mathrm{~Hz}, 1 \mathrm{H}), 1.34$ (s, $3 \mathrm{H}) ;{ }^{13} \mathrm{C} \mathrm{NMR}\left(151 \mathrm{MHz}, \mathrm{CDCl}_{3}\right) \delta 205.9,181.8,170.3,154.8,149.5,138.2,134.2,130.8$, $129.9,128.5,67.7,61.2,52.1,49.9,45.5,43.2,34.8,27.1,26.4$; IR (KBr): $v=2919,2849$, $1706,1652,1614,1598,1261,879,807 \mathrm{~cm}^{-1}$; HRMS (ESI, $m / z$ ): calcd for $\mathrm{C}_{19} \mathrm{H}_{18} \mathrm{Cl}_{3} \mathrm{O}_{2}$ $[\mathrm{M}+\mathrm{H}]^{+}$383.0367, found 383.0364.

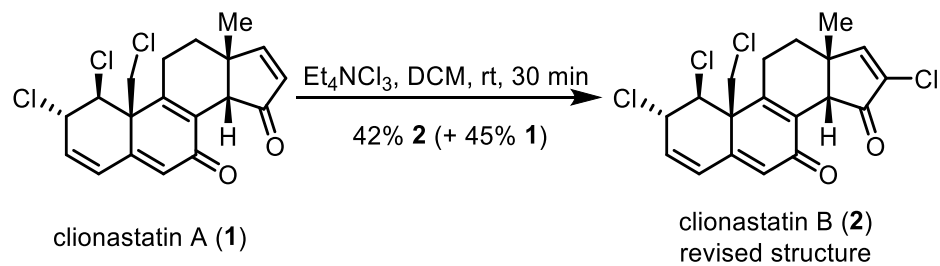

To a stirred solution of $\mathrm{Et}_{4} \mathrm{NCl}_{3}(54.0 \mathrm{mg}, 0.23 \mathrm{mmol}, 8.0$ equiv) in $1.0 \mathrm{~mL} \mathrm{DCM}$ at room temperature was added a solution of $1(11.0 \mathrm{mg}, 0.03 \mathrm{mmol}, 1.0$ equiv) in $1.0 \mathrm{~mL}$ DCM slowly. The reaction mixture was stirred for $30 \mathrm{~min}$ before it was quenched with aq. Sat. $\mathrm{NaHCO}_{3}(2 \mathrm{~mL})$. The reaction mixture was extracted with DCM $(3 \times 5 \mathrm{~mL})$, and the combined organic layers were washed with brine $(5 \mathrm{~mL})$, dried over anhydrous $\mathrm{Na}_{2} \mathrm{SO}_{4}$ and filtered. The volatiles were removed under vacuum, and the residue was purified by flash column chromatography with petroleum ether/EtOAc $(7: 1 \rightarrow 6: 1)$ to give clionastatin B (2, $5.1 \mathrm{mg}, 42 \%)$ and clionastatin A (1, $4.9 \mathrm{mg}, 45 \%)$ as white solids.

Clionastatin B: mp: $218.1-220.1{ }^{\circ} \mathrm{C}$; TLC (petroleum ether:EtOAc, 3:1 v/v): $R_{f}=0.50$; $[\alpha]_{\mathrm{D}}^{25}+54.3\left(c 0.02, \mathrm{CHCl}_{3}\right) ;{ }^{1} \mathrm{H} \mathrm{NMR}\left(600 \mathrm{MHz}, \mathrm{CDCl}_{3}\right) \delta 7.32(\mathrm{~s}, 1 \mathrm{H}), 6.53(\mathrm{~s}, 1 \mathrm{H}), 6.47$ $(\mathrm{dd}, J=10.0,2.1 \mathrm{~Hz}, 1 \mathrm{H}), 6.08(\mathrm{dd}, J=10.0,2.5 \mathrm{~Hz}, 1 \mathrm{H}), 4.71(\mathrm{dt}, J=9.0,2.5 \mathrm{~Hz}, 1 \mathrm{H})$, $4.25(\mathrm{~d}, J=11.2 \mathrm{~Hz}, 1 \mathrm{H}), 4.20(\mathrm{~d}, J=9.0 \mathrm{~Hz}, 1 \mathrm{H}), 4.12(\mathrm{~s}, 1 \mathrm{H}), 3.76(\mathrm{~d}, J=11.2 \mathrm{~Hz}, 1 \mathrm{H})$, $2.73(\mathrm{dt}, J=17.1,3.2 \mathrm{~Hz}, 1 \mathrm{H}), 2.09$ (ddd, $J=17.1,13.3,3.5 \mathrm{~Hz}, 1 \mathrm{H}), 1.96$ (dt, $J=13.5$, 
$3.2 \mathrm{~Hz}, 1 \mathrm{H}), 1.53(\mathrm{td}, J=13.5,3.2 \mathrm{~Hz}, 1 \mathrm{H}), 1.37(\mathrm{~s}, 3 \mathrm{H}) ;{ }^{13} \mathrm{C} \mathrm{NMR}\left(151 \mathrm{MHz}, \mathrm{CDCl}_{3}\right) \delta$ 197.3, 181.4, 163.0, 155.4, 149.7, 137.6, 135.5, 131.1, 129.8, 128.5, 67.7, 61.1, 52.3, 49.1, 43.2, 43.1, 35.0, 27.4, 26.5; IR (KBr): $v=2961,2924,1723,1655,1617,1601,1453,1261$, $807 \mathrm{~cm}^{-1}$; HRMS (ESI, $m / z$ ): calcd for $\mathrm{C}_{19} \mathrm{H}_{16} \mathrm{Cl}_{4} \mathrm{O}_{2}[\mathrm{M}+\mathrm{H}]^{+} 416.9977$, found 416.9972 . 


\section{NMR comparison of synthetic and natural clionastatins A and B}

${ }^{1} \mathrm{H}$ NMR data comparison of synthetic and natural clionastatin $\mathrm{A}$ in $\mathrm{CDCl}_{3}$

\begin{tabular}{|c|c|c|c|}
\hline Position & $\begin{array}{l}\text { Natural clionastatin } \mathrm{A}^{a} \\
\left(500 \mathrm{MHz} \text { in } \mathrm{CDCl}_{3}\right)\end{array}$ & $\begin{array}{c}\text { Synthetic clionastatin A } \\
\left(600 \mathrm{MHz} \text { in } \mathrm{CDCl}_{3}\right)\end{array}$ & $\Delta \delta(\mathrm{ppm})$ \\
\hline 1 & $4.20(\mathrm{~d}, 9.0)$ & $4.19(\mathrm{~d}, 9.0)$ & -0.01 \\
\hline 2 & $4.71(\mathrm{dd}, 9.0,2.0)$ & $4.71(\mathrm{dt}, 9.0,2.4)$ & 0 \\
\hline 3 & $6.06(\mathrm{dd}, 10.0,2.0)$ & $6.06(\mathrm{dd}, 9.9,2.4)$ & 0 \\
\hline 4 & $6.46(d, 10.0)$ & 6.47 (ddd, 9.9, 2.2, 0.9) & 0.01 \\
\hline 6 & $6.53(\mathrm{~s})$ & $6.53(\mathrm{~s})$ & 0 \\
\hline & $2.69(\mathrm{ddd}, 17.1,3.3,2.0)$ & $2.69(\mathrm{dt}, 17.1,3.2)$ & 0 \\
\hline & $2.08(\mathrm{ddd}, 17.1,15.2,3.3)$ & $2.08(\mathrm{ddd}, 17.1,13.3,3.5)$ & 0 \\
\hline & $1.92(\mathrm{ddd}, 15.2,3.3,2.0)$ & $1.92(\mathrm{dt}, 13.3,3.2)$ & 0 \\
\hline & $1.48(\mathrm{ddd}, 15.2,15.2,3.3)$ & $1.50(\mathrm{td}, 13.3,3.2)$ & 0.02 \\
\hline 14 & $3.99(\mathrm{~s})$ & $3.99(\mathrm{~s})$ & 0 \\
\hline 16 & $6.31(\mathrm{~d}, 6.5)$ & $6.31(\mathrm{~d}, 5.6)$ & 0 \\
\hline 17 & $7.40(\mathrm{~d}, 6.5)$ & $7.39(\mathrm{~d}, 5.6)$ & -0.01 \\
\hline 18 & $1.32(\mathrm{~s})$ & $1.34(\mathrm{~s})$ & 0.02 \\
\hline \multirow{2}{*}{19} & $3.76(\mathrm{~d}, 11.3)$ & $3.76(\mathrm{~d}, 11.2)$ & 0 \\
\hline & $4.24(\mathrm{~d}, 11.3)$ & $4.24(\mathrm{~d}, 11.2)$ & 0 \\
\hline
\end{tabular}

${ }^{a}$ Fattorusso, E.; Taglialatela-Scafati, O.; Petrucci, F.; Bavestrello, G.; Calcinai, B.; Cerrano, C.; Di Meglio, P.; Ianaro, A. Org. Lett. 2004, 6, 1633-1635. 


\section{${ }^{13} \mathrm{C}$ NMR data comparison of synthetic and natural clionastatin $\mathrm{A}$ in $\mathrm{CDCl}_{3}$}

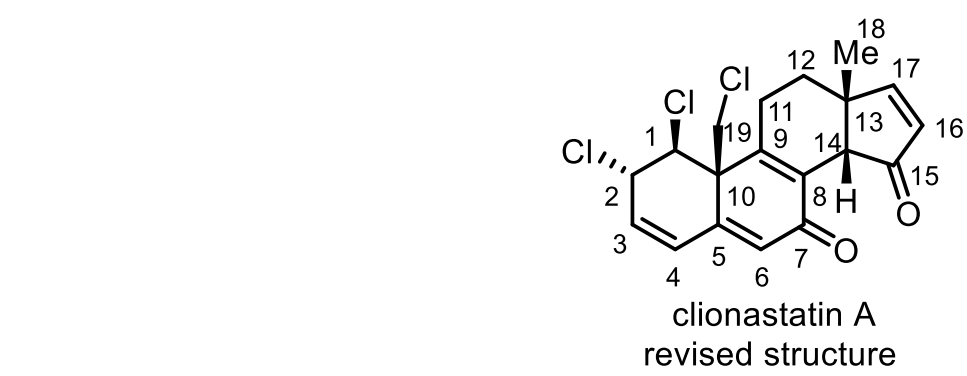

\begin{tabular}{|c|c|c|c|}
\hline Position & $\begin{array}{c}\text { Natural clionastatin } \mathrm{A}^{a} \\
\left(126 \mathrm{MHz} \text { in } \mathrm{CDCl}_{3}\right)\end{array}$ & $\begin{array}{c}\text { Synthetic clionastatin A } \\
\left(151 \mathrm{MHz} \text { in } \mathrm{CDCl}_{3}\right)\end{array}$ & $\begin{array}{c}\Delta \delta \\
(\mathrm{ppm})\end{array}$ \\
\hline 1 & 67.5 & 67.7 & 0.2 \\
\hline 2 & 61.3 & 61.2 & -0.1 \\
\hline 3 & 132.8 & 130.8 & 2 \\
\hline 4 & 128.6 & 128.5 & -0.1 \\
\hline 5 & 149.3 & 149.5 & 0.2 \\
\hline 6 & 130.1 & 129.9 & -0.2 \\
\hline 7 & 181.6 & 181.8 & 0.2 \\
\hline 8 & 138.5 & 138.2 & -0.3 \\
\hline 9 & 154.9 & 154.8 & -0.1 \\
\hline 10 & 52.3 & 52.1 & -0.2 \\
\hline 11 & 27.3 & 27.1 & -0.2 \\
\hline 12 & 35.0 & 34.8 & -0.2 \\
\hline 13 & 45.5 & 45.5 & 0 \\
\hline 14 & 50.1 & 49.9 & -0.2 \\
\hline 15 & 205.9 & 205.9 & 0 \\
\hline 16 & 134.0 & 134.2 & 0.2 \\
\hline 17 & 170.5 & 170.3 & -0.2 \\
\hline 18 & 27.0 & 26.4 & -0.6 \\
\hline 19 & 43.6 & 43.2 & -0.4 \\
\hline
\end{tabular}

${ }^{a}$ Fattorusso, E.; Taglialatela-Scafati, O.; Petrucci, F.; Bavestrello, G.; Calcinai, B.; Cerrano, C.; Di Meglio, P.; Ianaro, A. Org. Lett. 2004, 6, 1633-1635. 
${ }^{1} \mathrm{H}$ NMR data comparison of synthetic and natural clionastatin $\mathrm{B}$ in $\mathrm{CDCl}_{3}$

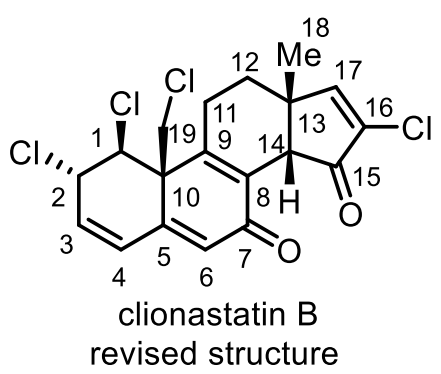

\begin{tabular}{|c|c|c|c|}
\hline Position & $\begin{array}{l}\text { Natural clionastatin } \mathrm{B}^{a} \\
\left(500 \mathrm{MHz} \text { in } \mathrm{CDCl}_{3}\right)\end{array}$ & $\begin{array}{l}\text { Synthetic clionastatin B } \\
\left(600 \mathrm{MHz} \text { in } \mathrm{CDCl}_{3}\right)\end{array}$ & $\begin{array}{c}\Delta \delta \\
(\mathrm{ppm})\end{array}$ \\
\hline 1 & $4.20(\mathrm{~d}, 9.0)$ & $4.20(\mathrm{~d}, 9.0)$ & 0 \\
\hline 2 & $4.71(\mathrm{dd}, 9.0,2.0)$ & $4.71(\mathrm{dt}, 9.0,2.4)$ & 0 \\
\hline 3 & $6.08(\mathrm{dd}, 10.0,2.0)$ & $6.08(\mathrm{dd}, 9.9,2.4)$ & 0 \\
\hline 4 & $6.46(\mathrm{~d}, 10.0)$ & $6.46(\mathrm{dd}, 9.9,2.2)$ & 0 \\
\hline 6 & $6.53(\mathrm{~s})$ & $6.53(\mathrm{~s})$ & 0 \\
\hline \multirow{2}{*}{11} & $2.72(\mathrm{ddd}, 17.1,3.3,2.0)$ & $2.73(\mathrm{dt}, 17.1,3.2)$ & 0.01 \\
\hline & $2.08(\mathrm{ddd}, 17.1,15.2,3.3)$ & $2.08(\mathrm{ddd}, 17.1,13.3,3.5)$ & 0 \\
\hline \multirow{2}{*}{12} & $1.98(\mathrm{ddd}, 15.2,3.3,2.0)$ & $1.92(\mathrm{dt}, 13.3,3.2)$ & 0 \\
\hline & $1.48(\mathrm{ddd}, 15.2,15.2,3.3)$ & $1.50(\mathrm{td}, 13.3,3.2)$ & 0.02 \\
\hline 14 & $4.12(\mathrm{~s})$ & $4.12(\mathrm{~s})$ & 0 \\
\hline 16 & $6.31(\mathrm{~d}, 6.5)$ & $6.31(\mathrm{~d}, 5.6)$ & 0 \\
\hline 17 & $7.40(\mathrm{~d}, 6.5)$ & $7.39(\mathrm{~d}, 5.6)$ & -0.01 \\
\hline 18 & $1.32(\mathrm{~s})$ & $1.37(\mathrm{~s})$ & 0.05 \\
\hline \multirow{2}{*}{19} & $3.74(\mathrm{~d}, 11.3)$ & $3.76(\mathrm{~d}, 11.2)$ & 0 \\
\hline & $4.24(\mathrm{~d}, 11.3)$ & $4.25(\mathrm{~d}, 11.2)$ & 0.01 \\
\hline
\end{tabular}

${ }^{a}$ Fattorusso, E.; Taglialatela-Scafati, O.; Petrucci, F.; Bavestrello, G.; Calcinai, B.; Cerrano, C.; Di Meglio, P.; Ianaro, A. Org. Lett. 2004, 6, 1633-1635. 


\section{${ }^{13} \mathrm{C}$ NMR data comparison of synthetic and natural clionastatin $\mathrm{B}$ in $\mathrm{CDCl}_{3}$}

\begin{tabular}{|c|c|c|c|}
\hline Position & $\begin{array}{l}\text { Natural clionastatin } \mathrm{B}^{a} \\
\left(126 \mathrm{MHz} \text { in } \mathrm{CDCl}_{3}\right)\end{array}$ & $\begin{array}{l}\text { Synthetic clionastatin B } \\
\left(151 \mathrm{MHz} \text { in } \mathrm{CDCl}_{3}\right)\end{array}$ & $\begin{array}{c}\Delta \delta \\
(\mathrm{ppm})\end{array}$ \\
\hline 1 & 67.6 & 67.7 & 0.1 \\
\hline 2 & 60.9 & 61.1 & 0.2 \\
\hline 3 & 131.1 & 131.1 & 0 \\
\hline 4 & 128.8 & 128.5 & 0.3 \\
\hline 5 & 149.7 & 149.7 & 0 \\
\hline 6 & 130.1 & 129.8 & -0.3 \\
\hline 7 & 181.4 & 181.4 & 0 \\
\hline 8 & 137.5 & 137.6 & 0.1 \\
\hline 9 & 155.4 & 155.4 & 0 \\
\hline 10 & 52.2 & 52.3 & 0.1 \\
\hline 11 & 26.8 & 26.5 & -0.3 \\
\hline 12 & 34.6 & 35.0 & 0.4 \\
\hline 13 & 43.1 & 43.2 & 0.1 \\
\hline 14 & 48.9 & 49.1 & 0.3 \\
\hline 15 & 197.3 & 197.3 & 0 \\
\hline 16 & 124.6 & 135.5 & 9.9 \\
\hline 17 & 163.5 & 163.0 & -0.5 \\
\hline 18 & 27.0 & 27.4 & 0.4 \\
\hline 19 & 42.8 & 43.1 & 0.3 \\
\hline
\end{tabular}

${ }^{a}$ Fattorusso, E.; Taglialatela-Scafati, O.; Petrucci, F.; Bavestrello, G.; Calcinai, B.; Cerrano, C.; Di Meglio, P.; Ianaro, A. Org. Lett. 2004, 6, 1633-1635. 


\section{${ }^{1} \mathrm{H}$ NMR spectra comparison of natural and synthetic clionastatin $\mathrm{A}$ in $\mathrm{CDCl}_{3}$}
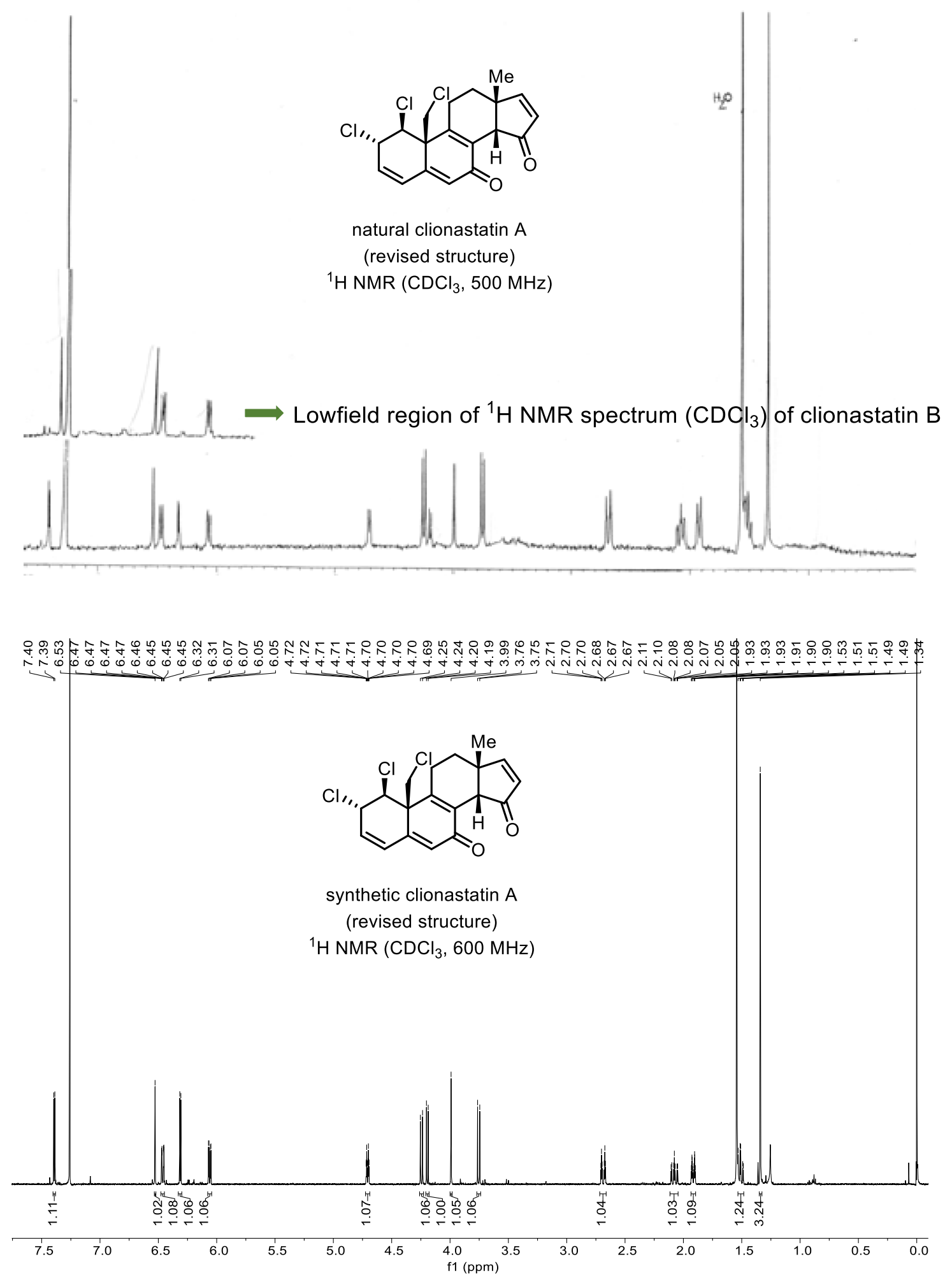
HSQC spectra comparison of synthetic and natural clionastatin $\mathrm{A}$ in $\mathrm{CDCl}_{3}$
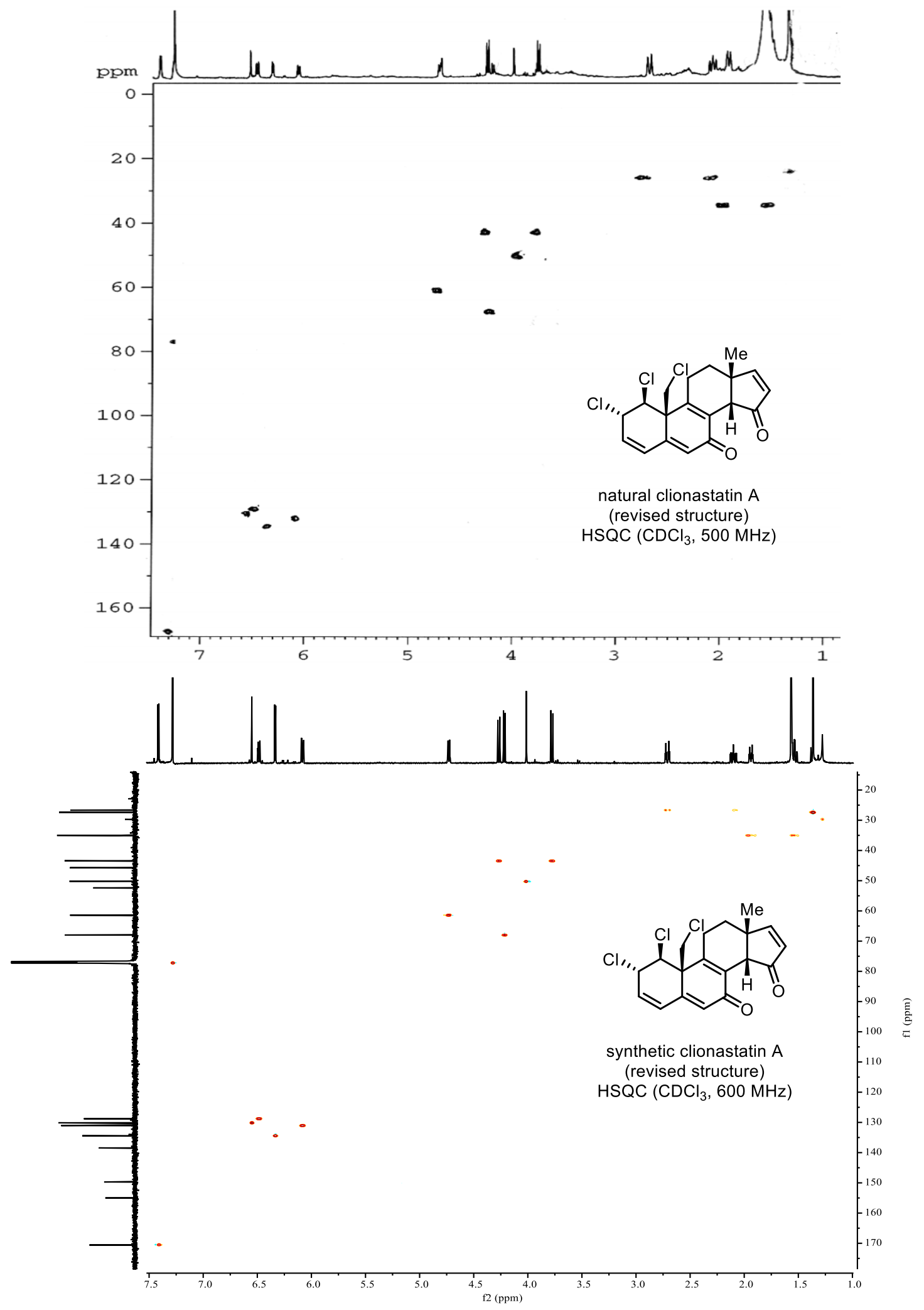


\section{${ }^{1} \mathrm{H}$ NMR spectra comparison of natural and synthetic clionastatin $\mathrm{B}$ in $\mathrm{CDCl}_{3}$}

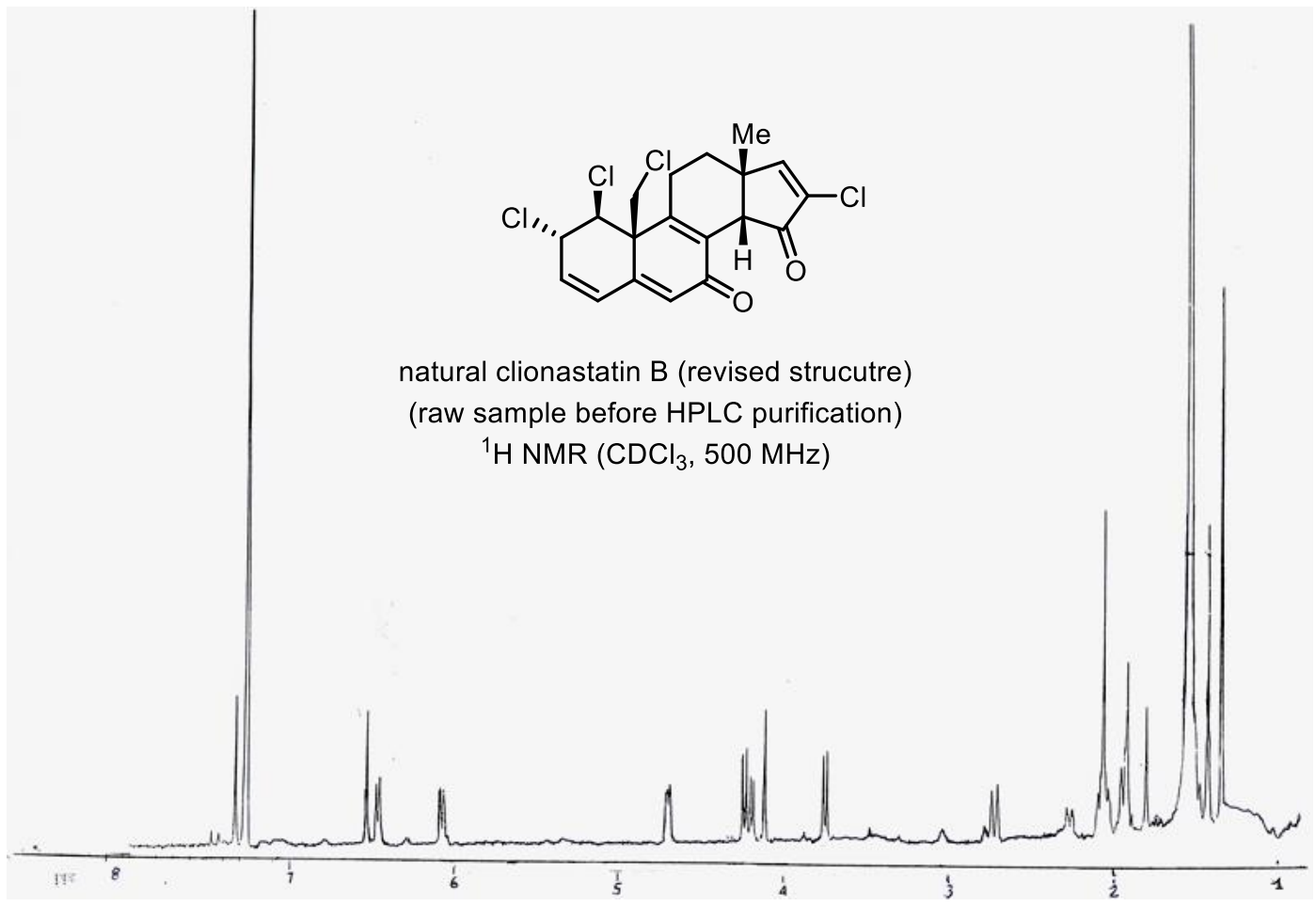

Note: The ${ }^{1} \mathrm{H}$ NMR spectrum of natural clionastatin B (raw sample before HPLC purification) was kindly provided by Prof. Orazio Taglialatela-Scafati (University of Naples Federico II).

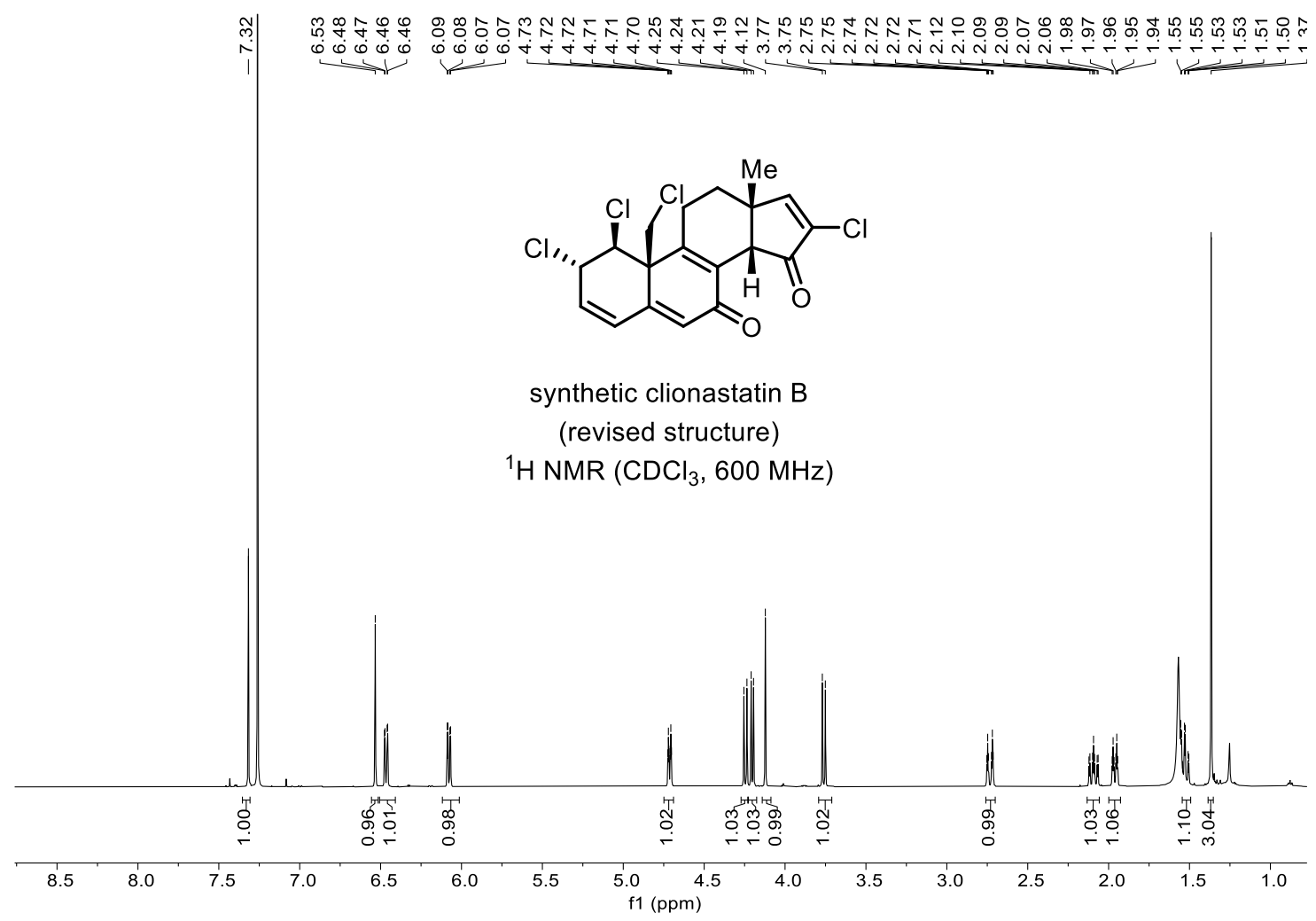




\section{Discussion of some discrepancies in the ${ }^{13} \mathrm{C}$ NMR data}

The ${ }^{13} \mathrm{C}$ NMR spectra of natural clionastatins were not registered, possibly because only approximately $1 \mathrm{mg}$ of each compound was isolated. The complete ${ }^{13} \mathrm{C}$ NMR assignment was deduced through inspection of the 2D NMR spectra HSQC and HMBC (personal communication with one of the isolation chemists Prof. Orazio Taglialatela-Scafati from University of Naples Federico II). Therefore, the accuracy of the reported ${ }^{13} \mathrm{C}$ NMR data remains to be determined.

\section{1) Discussion of one discrepancy (at C3) in the ${ }^{13} \mathrm{C}$ NMR data of clionastatin A}

The ${ }^{1} \mathrm{H}$ NMR spectra of synthetic and natural clionastatin A are identical and the HSQC spectra are practically superimposable, suggesting that our synthetic sample was identical to the natural product. As for the difference of 2 ppm at C3 (reported: 132.8 ppm, ours: $130.8 \mathrm{ppm})$ in the ${ }^{13} \mathrm{C}$ NMR data, we took a close look at the HSQC spectrum of the natural sample and found that the C3 chemical shift should be around $131 \mathrm{ppm}$, not $133 \mathrm{ppm}$ as reported (Figure S1). Therefore, this discrepancy is most likely due to a typo error.

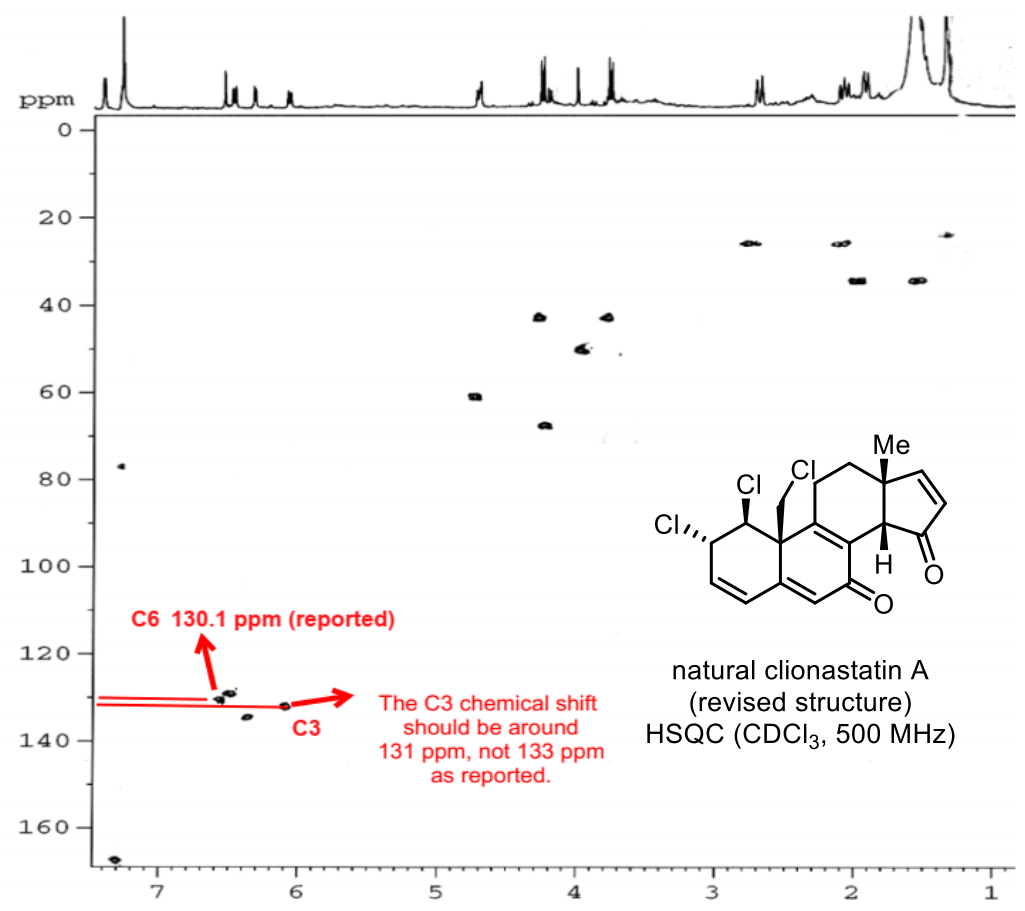

Figure S1. HSQC spectrum of the natural clionastatin A 
In addition, a further support comes from the reported $\mathrm{C} 3$ chemical shift of clionastatin B (reported: $131.1 \mathrm{ppm}$, ours: $131.1 \mathrm{ppm}$, Figure S2). We surmised that the C3 chemical shifts of clionastatins A and B should be very similar, because the presence of an additional chlorine atom on the D ring should not significantly affect the resonance at $\mathrm{C} 3$.

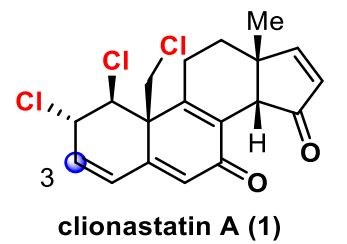

The $\mathbf{C} 3$ chemical shift: reported $132.8 \mathrm{ppm}$; synthetic $130.8 \mathrm{ppm}$.

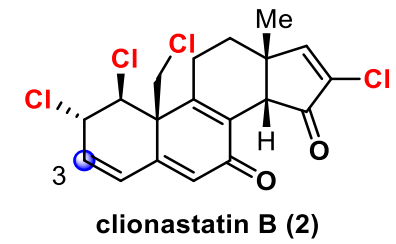

The $\mathbf{C} 3$ chemical shift: reported $131.1 \mathrm{ppm}$; synthetic $131.1 \mathrm{ppm}$.

Figure S2. The C3 chemical shift of clionastatins A and B

\section{2) Discussion of one discrepancy (at $\mathrm{C} 16$ ) in the ${ }^{13} \mathrm{C}$ NMR data of clionastatin $B$}

The ${ }^{1} \mathrm{H}$ NMR spectra of synthetic and natural clionastatin B are identical, suggesting that our synthetic sample was identical to the natural product. However, there is a large discrepancy (9.9 ppm) of the ${ }^{13} \mathrm{C}$ NMR data between the synthetic and natural samples at C16 (reported: $124.6 \mathrm{ppm}$, ours: $135.5 \mathrm{ppm}$ ). Since the C16 of clionastatin B is a fully substituted sp2 carbon, the HMBC correlations between $\mathrm{C} 16$ and the nearby hydrogens are expected to be very weak, which makes the reporting of the C16 chemical shift from the HMBC spectrum very difficult. In fact, in the HMBC spectrum of our synthetic clionastatin B (Figure S2), only correlations between C-16 and H-18/H-12 are observed. The HMBC correlations between $\mathrm{C}-16$ and other nearby hydrogens (such as: $\mathrm{H}-17$ and $\mathrm{H}-14$ ) are not observed. Since only $1.3 \mathrm{mg}$ of natural clionastatin B was isolated in 2004, it is well possible that some feeble cross-peaks in the HMBC spectra had been misinterpreted, which led to the incorrect reporting of the ${ }^{13} \mathrm{C}$ NMR data at $\mathrm{C} 16$.

[We gratefully thank Prof. Orazio Taglialatela-Scafati for his generous help and insightful discussions on the discrepancies in the ${ }^{13} \mathrm{C} N M R$ data.] 


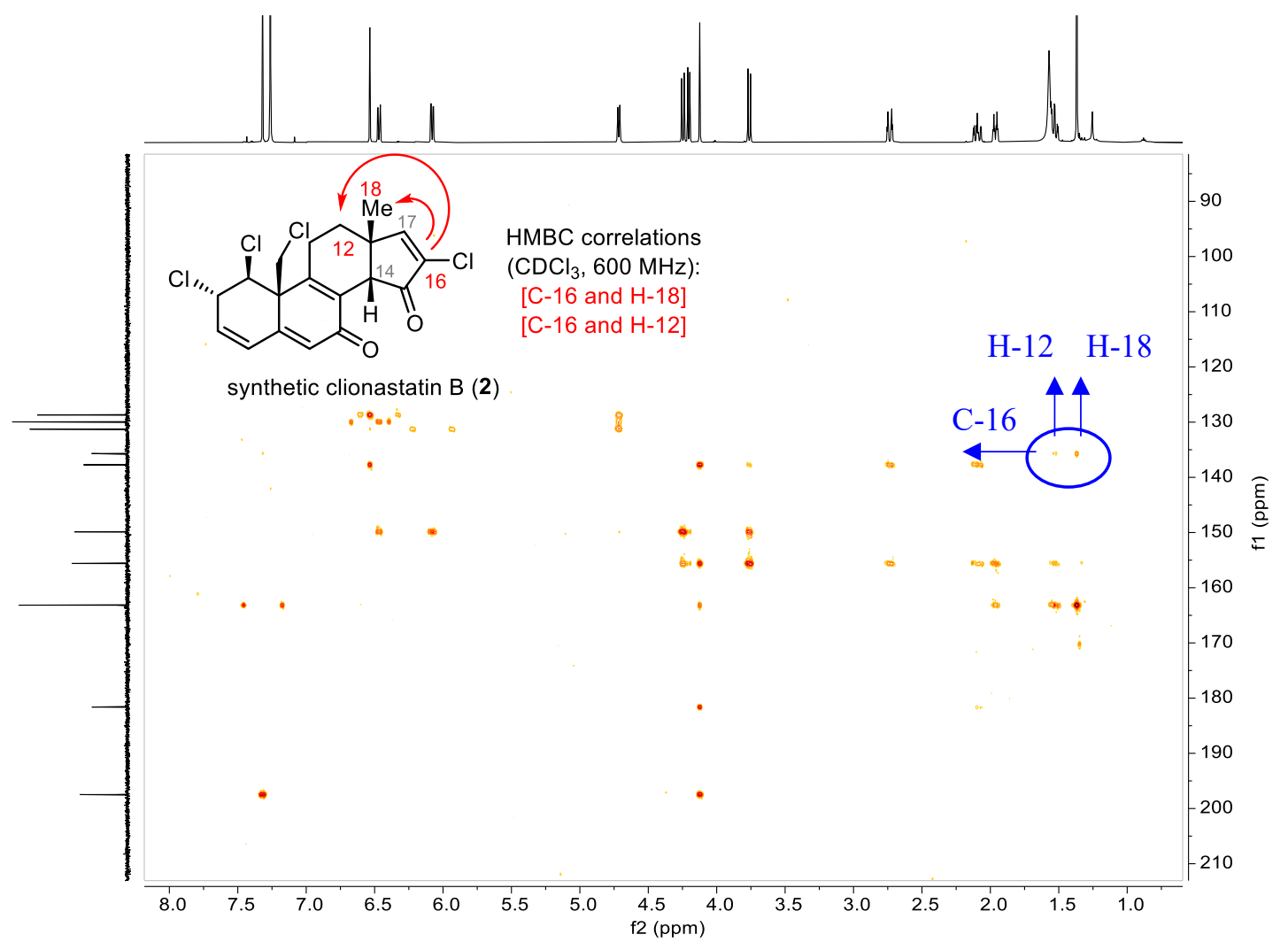

Figure S2. The HMBC spectrum of synthetic clionastatin B

Moreover, the $\mathrm{C} 16$ chemical shift of clionastatin A was reported to be $134.0 \mathrm{ppm}$ (ours: $134.2 \mathrm{ppm}$ ), while that of clionastatin B was reported to be $124.6 \mathrm{ppm}$ (ours: $135.5 \mathrm{ppm}$ ). Due to the electron-withdrawing property of the chlorine atom, we surmised that replacing $\mathrm{C} 16-\mathrm{H}$ by the chlorine atom should make the $\mathrm{C} 16$ chemical shift of clionastatin B larger than that of clionastatin A.
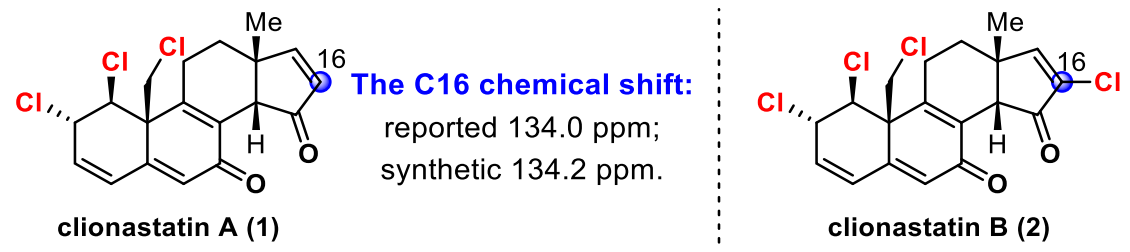

The C16 chemical shift: reported $124.6 \mathrm{ppm}$; synthetic $135.5 \mathrm{ppm}$.

Figure S3. The $\mathrm{C} 16$ chemical shift of clionastatins A and B 


\section{Structural revision of clionastatins}

As shown in Figure S4 and S5, NOE spectroscopy of synthetic clionastatins A (1) and B (2) showed strong correlation between the $\mathrm{C} 18-\mathrm{Me}$ and the $\mathrm{C} 14-\mathrm{H}$, which indicates that the $\mathrm{C} 14-\mathrm{H}$ was actually in the $\beta$-configuration rather than the originally proposed $\alpha$ configuration. This configuration was unambiguously confirmed by X-ray crystallographic analysis of synthetic clionastatins A (1) (Figure S6). That is, the C/D-ring system of the clionastatins is in fact in a cis-fused arrangement. [Note: In the isolation paper, key ROESY correlation between $\mathrm{C} 12-\mathrm{H}$ and $\mathrm{C} 14-\mathrm{H}$ was observed for clionastatin $\mathrm{A}$. It was likely that the ROESY spectrum (and the specific parameters used) minimized the intensity of the crucial cross-peak between $\mathrm{C} 18-\mathrm{Me}$ and $\mathrm{C} 14-\mathrm{H}$, and some minor cross-peaks were misinterpreted (personal communication with Prof. Orazio Taglialatela-Scafati).]

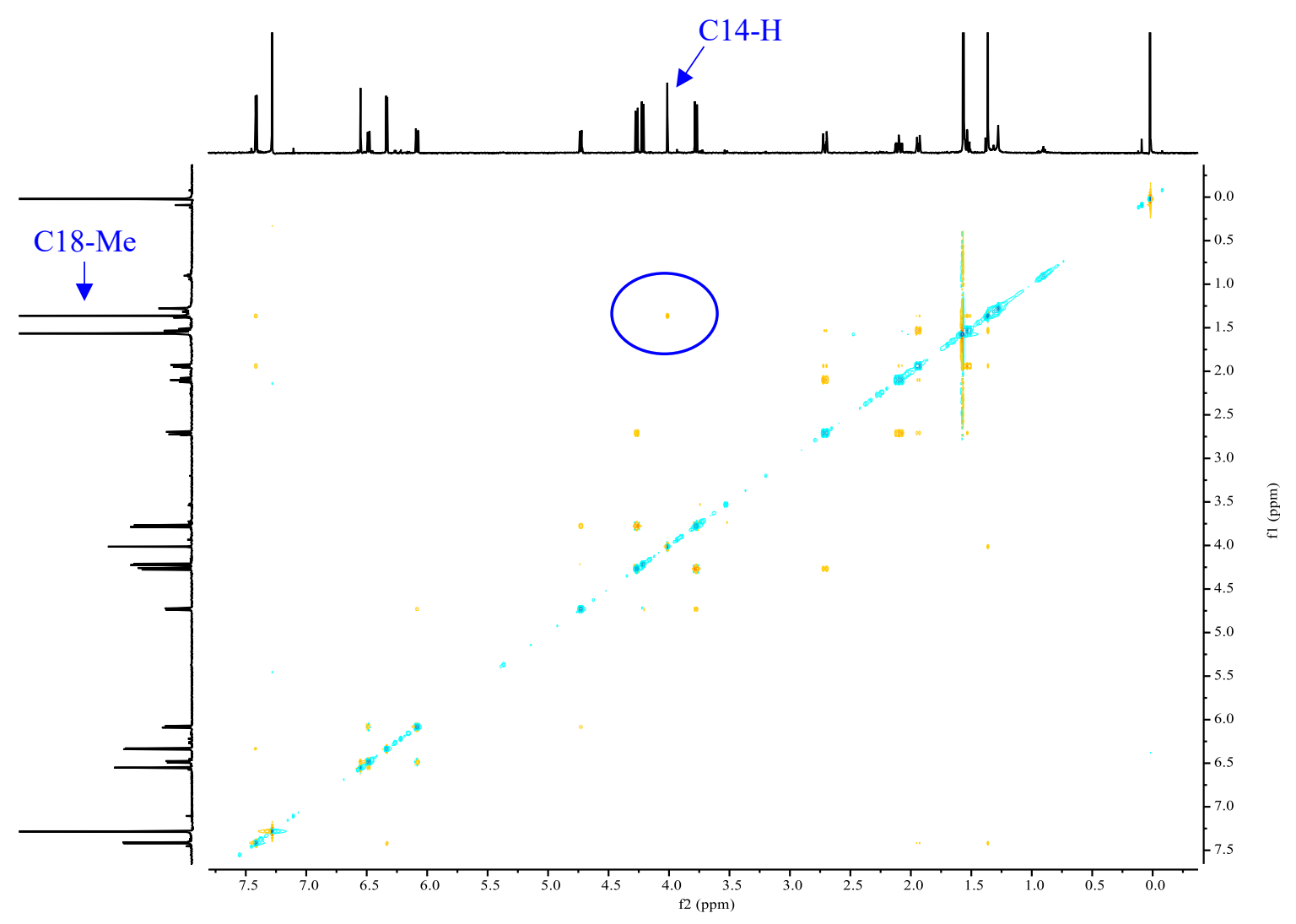



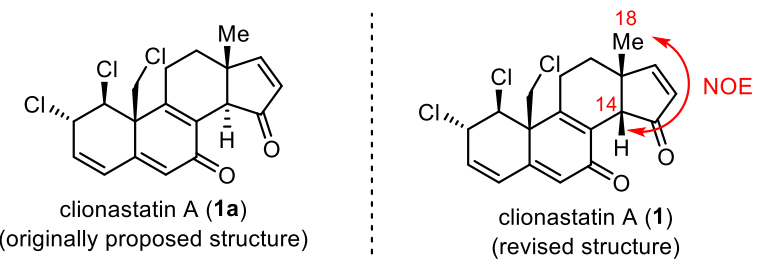

Figure S4. The NOE spectrum of synthetic clionastatin A

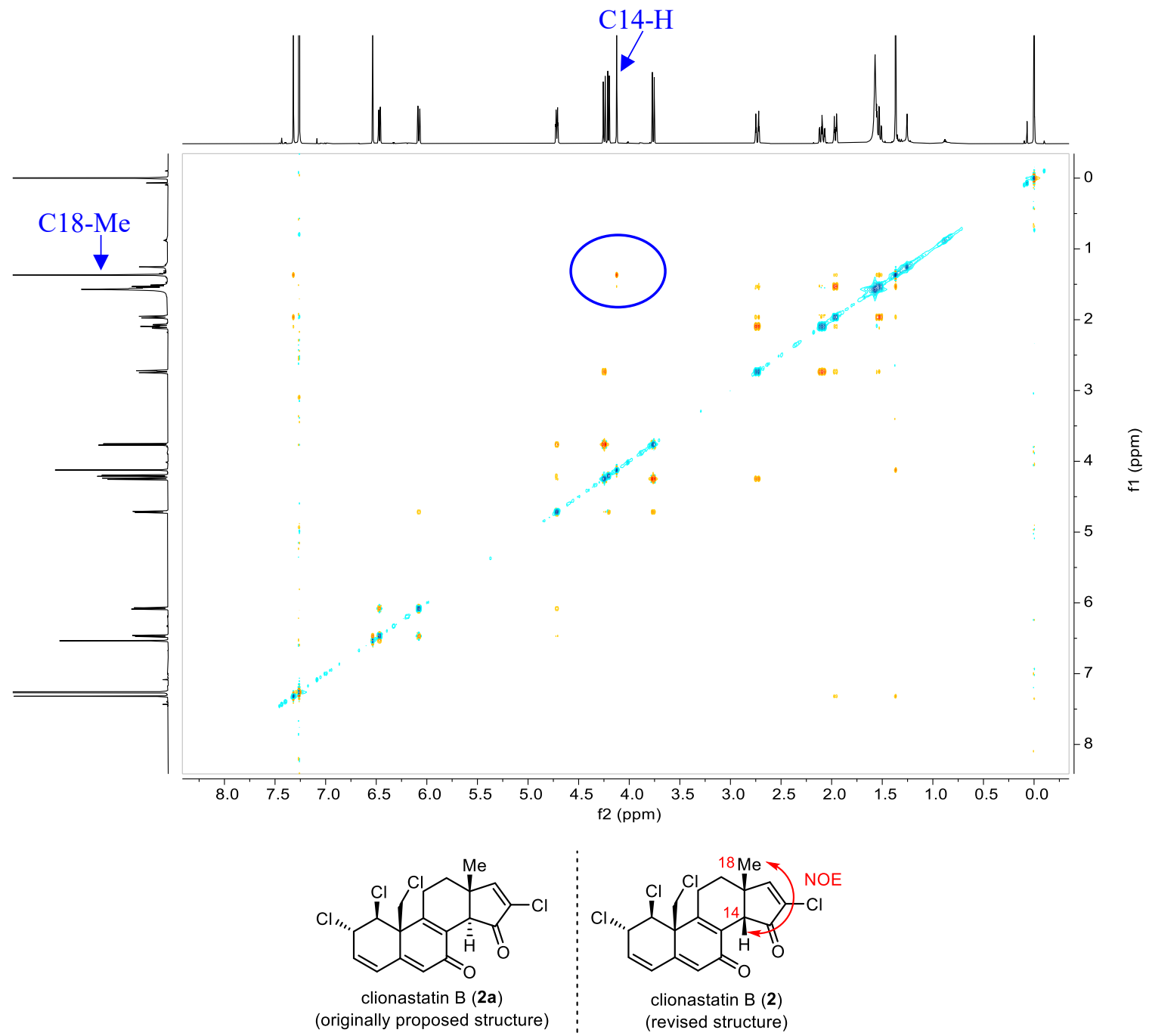

Figure S5. The NOE spectrum of synthetic clionastatin B

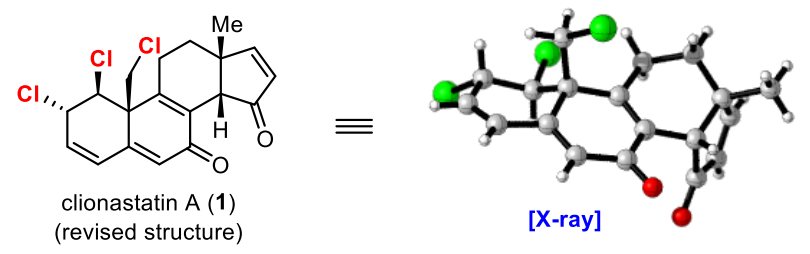

Figure S6. The X-ray structure of synthetic clionastatin A (CCDC 2092061) 


\section{X-ray crystallographic data for compounds 1, 18, 24, 25 and 29}

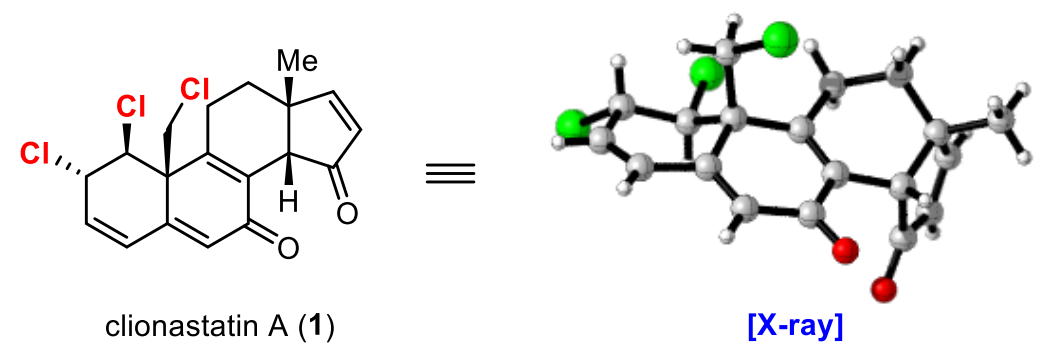

Table S4. Crystal data and structure refinement for CCDC 2092061

Identification code

Empirical formula

Formula weight

Temperature

Wavelength

Crystal system

Space group

Unit cell dimensions

Volume

Z

Density (calcd)

Absorption coefficient

$\mathrm{F}(000)$

Crystal size

Theta range for data collection

Index ranges

Reflections collected

Independent reflections

Completeness to theta $=25.242^{\circ}$

Absorption correction

Max. and min. transmission

Refinement method

Data / restraints / parameters

Goodness-of-fit on $\mathrm{F}^{2}$

Final $\mathrm{R}$ indices [I $>2 \operatorname{sigma}(\mathrm{I})]$

$\mathrm{R}$ indices (all data)

Absolute structure parameter

Extinction coefficient

Largest diff. peak and hole
CCDC 2092061

C19 H17 Cl3 O2

383.67

293(2) K

$0.71073 \AA$

Monoclinic

P 21

$\mathrm{a}=11.9094(15) \AA$

$\mathrm{a}=90^{\circ}$.

$\mathrm{b}=11.9294(15) \AA$ $\mathrm{b}=92.879(4)^{\circ}$.

$\mathrm{c}=12.7681(16) \AA$

1811.7(4) $\AA^{3}$

4

$1.407 \mathrm{Mg} / \mathrm{m}^{3}$

$0.514 \mathrm{~mm}^{-1}$

792

$0.190 \times 0.100 \times 0.080 \mathrm{~mm}^{3}$

2.418 to $25.994^{\circ}$.

$-14<=\mathrm{h}<=14,-14<=\mathrm{k}<=14,-13<=1<=15$

21555

$7082[\mathrm{R}($ int $)=0.0315]$

$99.6 \%$

Semi-empirical from equivalents

0.7456 and 0.6244

Full-matrix least-squares on $\mathrm{F}^{2}$

7082 / 1 / 435

1.060

$\mathrm{R} 1=0.0394, \mathrm{wR} 2=0.0758$

$\mathrm{R} 1=0.0602, \mathrm{wR} 2=0.0868$

$0.00(2)$

$\mathrm{n} / \mathrm{a}$

0.179 and -0.249 e. $\AA^{-3}$ 


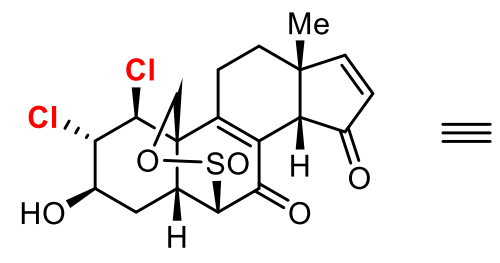

18

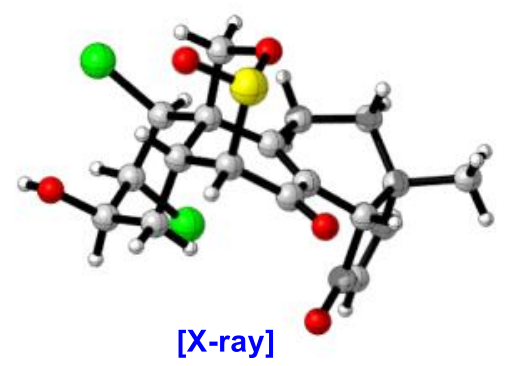

[X-ray]

Table S5. Crystal data and structure refinement for CCDC 2092062

Identification code

Empirical formula

Formula weight

Temperature

Wavelength

Crystal system

Space group

Unit cell dimensions

Volume

$\mathrm{Z}$

Density (calcd)

Absorption coefficient

$\mathrm{F}(000)$

Crystal size

Theta range for data collection

Index ranges

Reflections collected

Independent reflections

Completeness to theta $=25.242^{\circ}$

Absorption correction

Max. and min. transmission

Refinement method

Data / restraints / parameters

Goodness-of-fit on $\mathrm{F}^{2}$

Final $\mathrm{R}$ indices [I $>2 \operatorname{sigma}(\mathrm{I})]$

$\mathrm{R}$ indices (all data)

Absolute structure parameter

Extinction coefficient

Largest diff. peak and hole
CCDC 2092062

$\mathrm{C} 20 \mathrm{H} 22 \mathrm{Cl} 4 \mathrm{O} 5 \mathrm{~S}$

516.23

293(2) K

$0.71073 \AA$

Monoclinic

P 21

$\mathrm{a}=8.3009(3) \AA$ $\alpha=90^{\circ}$.

$\mathrm{B}=8.6917(3) \AA$ $\beta=97.2520(10)^{\circ}$.

$\mathrm{C}=15.3430(5) \AA$ 1098.13(7) $\AA^{3}$

2

$1.561 \mathrm{Mg} / \mathrm{m}^{3}$

$0.665 \mathrm{~mm}^{-1}$

532

$0.200 \times 0.170 \times 0.110 \mathrm{~mm}^{3}$

2.473 to $25.984^{\circ}$.

$-10<=\mathrm{h}<=10,-10<=\mathrm{k}<=10,-18<=1<=16$

16179

$4311[\mathrm{R}(\mathrm{int})=0.0284]$

$99.8 \%$

Semi-empirical from equivalents

0.7456 and 0.6642

Full-matrix least-squares on $\mathrm{F}^{2}$

4311 / 1 / 274

1.042

$\mathrm{R} 1=0.0489, \mathrm{wR} 2=0.1315$

$\mathrm{R} 1=0.0555, \mathrm{wR} 2=0.1380$

$0.03(2)$

$0.014(5)$

0.818 and -0.516 e. $\AA^{-3}$ 


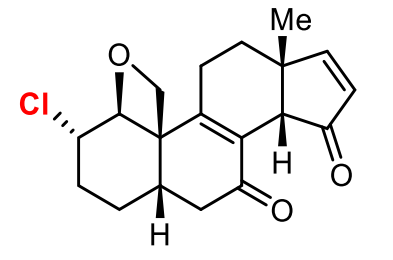

24

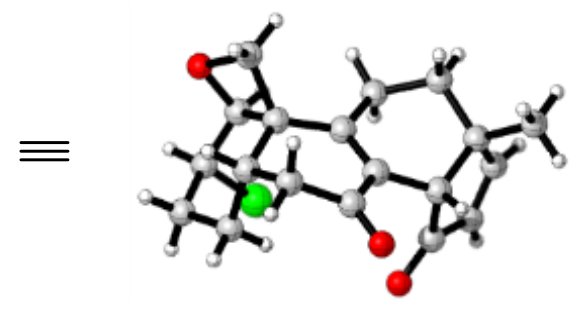

[X-ray]

Table S6. Crystal data and structure refinement for CCDC 2092063

Identification code

Empirical formula

Formula weight

Temperature

Wavelength

Crystal system

Space group

Unit cell dimensions

Volume

$\mathrm{Z}$

Density (calcd)

Absorption coefficient

$\mathrm{F}(000)$

Crystal size

Theta range for data collection

Index ranges

Reflections collected

Independent reflections

Completeness to theta $=67.679^{\circ}$

Absorption correction

Max. and min. transmission

Refinement method

Data / restraints / parameters

Goodness-of-fit on $\mathrm{F}^{2}$

Final $\mathrm{R}$ indices [I $>2 \operatorname{sigma}(\mathrm{I})]$

$\mathrm{R}$ indices (all data)

Absolute structure parameter

Extinction coefficient

Largest diff. peak and hole
CCDC 2092063

$\mathrm{C} 19 \mathrm{H} 21 \mathrm{Cl} \mathrm{O} 3$

332.81

293(2) K

$1.54178 \AA$

Monoclinic

P 21

$\mathrm{a}=10.4201(4) \AA$ $\alpha=90^{\circ}$.

$\mathrm{b}=14.1778(5) \AA$ $\beta=103.8440(10)^{\circ}$.

$\mathrm{c}=11.7663(4) \AA$

1687.79(11) $\AA^{3}$

$\gamma=90^{\circ}$.

4

$1.310 \mathrm{Mg} / \mathrm{m}^{3}$

$2.103 \mathrm{~mm}^{-1}$

704

$0.160 \times 0.130 \times 0.100 \mathrm{~mm}^{3}$

3.869 to $67.475^{\circ}$.

$-12<=\mathrm{h}<=12,-16<=\mathrm{k}<=15,-13<=\mathrm{l}<=14$

35741

$5955[\mathrm{R}(\mathrm{int})=0.0462]$

$98.9 \%$

Semi-empirical from equivalents

0.7533 and 0.5442

Full-matrix least-squares on $\mathrm{F}^{2}$

$5955 / 1 / 418$

1.051

$\mathrm{R} 1=0.0411, \mathrm{wR} 2=0.1049$

$\mathrm{R} 1=0.0458, \mathrm{wR} 2=0.1106$

$0.040(6)$

$0.0126(15)$

0.256 and -0.342 e. $\AA^{-3}$ 


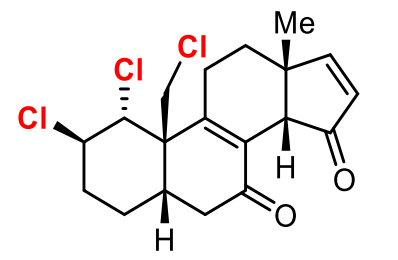

25

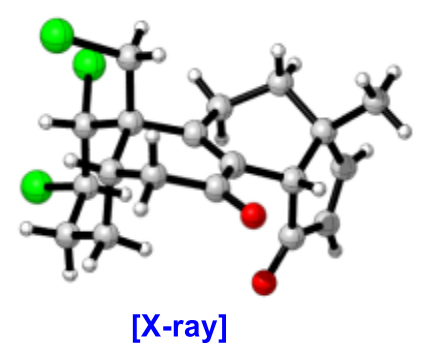

[X-ray]

\section{Table S7. Crystal data and structure refinement for CCDC 2092064}

Identification code

Empirical formula

Formula weight

Temperature

Wavelength

Crystal system

Space group

Unit cell dimensions

Volume

Z

Density (calcd)

Absorption coefficient

$\mathrm{F}(000)$

Crystal size

Theta range for data collection

Index ranges

Reflections collected

Independent reflections

Completeness to theta $=25.242^{\circ}$

Absorption correction

Max. and min. transmission

Refinement method

Data / restraints / parameters

Goodness-of-fit on $\mathrm{F}^{2}$

Final $\mathrm{R}$ indices [I $>2 \operatorname{sigma}(\mathrm{I})]$

$\mathrm{R}$ indices (all data)

Absolute structure parameter

Extinction coefficient

Largest diff. peak and hole
CCDC 2092064

$\mathrm{C} 19 \mathrm{H} 21 \mathrm{Cl} 3 \mathrm{O} 2$

387.71

293(2) K

$0.71073 \AA$

Orthorhombic

P 212121

$\mathrm{a}=7.4498(2) \AA$

$\alpha=90^{\circ}$.

$\mathrm{b}=12.7686(5) \AA$

$\beta=90^{\circ}$.

$\mathrm{c}=19.0483(6) \AA$

$\gamma=90^{\circ}$.

1811.94(10) $\AA^{3}$

4

$1.421 \mathrm{Mg} / \mathrm{m}^{3}$

$0.514 \mathrm{~mm}^{-1}$

808

$0.190 \times 0.150 \times 0.120 \mathrm{~mm}^{3}$

2.668 to $25.997^{\circ}$.

$-9<=\mathrm{h}<=9,-12<=\mathrm{k}<=15,-23<=1<=20$

9136

$3540[\mathrm{R}(\mathrm{int})=0.0205]$

$99.0 \%$

Semi-empirical from equivalents

0.7456 and 0.6334

Full-matrix least-squares on $\mathrm{F}^{2}$

3540 / 0 / 218

1.056

$\mathrm{R} 1=0.0295, \mathrm{wR} 2=0.0681$

$\mathrm{R} 1=0.0332, \mathrm{wR} 2=0.0707$

$0.00(2)$

$\mathrm{n} / \mathrm{a}$

0.195 and -0.204 e. $\AA^{-3}$ 


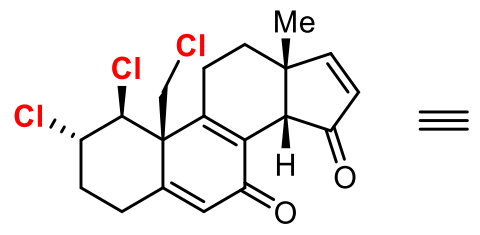

29

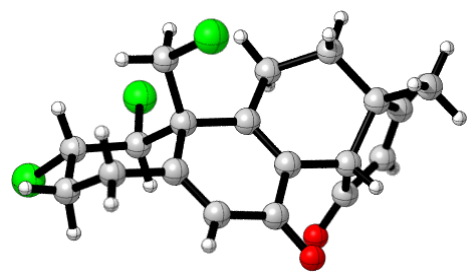

[X-ray]

Table S8. Crystal data and structure refinement for CCDC 2092065

Identification code

Empirical formula

Formula weight

Temperature

Wavelength

Crystal system

Space group

Unit cell dimensions

Volume

$\mathrm{Z}$

Density (calcd)

Absorption coefficient

$\mathrm{F}(000)$

Crystal size

Theta range for data collection

Index ranges

Reflections collected

Independent reflections

Completeness to theta $=25.242^{\circ}$

Absorption correction

Max. and min. transmission

Refinement method

Data / restraints / parameters

Goodness-of-fit on $\mathrm{F}^{2}$

Final R indices [I $>2 \operatorname{sigma}(\mathrm{I})]$

$\mathrm{R}$ indices (all data)

Absolute structure parameter

Extinction coefficient

Largest diff. peak and hole
CCDC 2092065

C19 $\mathrm{H} 19 \mathrm{Cl} 3 \mathrm{O} 2$

385.69

293(2) K

$0.71073 \AA$

Monoclinic

P 21

$\mathrm{a}=8.3483(3) \AA$

$\alpha=90^{\circ}$.

$\mathrm{b}=8.4665(3) \AA$

$\beta=104.4410(10)^{\circ}$.

$\mathrm{c}=12.9804(4) \AA$ $\gamma=90^{\circ}$.

888.48(5) $\AA^{3}$

2

$1.442 \mathrm{Mg} / \mathrm{m}^{3}$

$0.524 \mathrm{~mm}^{-1}$

400

$0.200 \times 0.150 \times 0.110 \mathrm{~mm}^{3}$

3.319 to $25.991^{\circ}$.

$-10<=\mathrm{h}<=10,-10<=\mathrm{k}<=10,-15<=\mathrm{l}<=15$

13900

$3440[\mathrm{R}(\mathrm{int})=0.0283]$

$98.1 \%$

Semi-empirical from equivalents

0.7456 and 0.6402

Full-matrix least-squares on $\mathrm{F}^{2}$

3440 / 1 / 218

1.051

$\mathrm{R} 1=0.0302, \mathrm{wR} 2=0.0761$

$\mathrm{R} 1=0.0318, \mathrm{wR} 2=0.0778$

$0.008(17)$

$\mathrm{n} / \mathrm{a}$

0.347 and -0.168 e. $\AA^{-3}$ 


\section{NMR spectra}

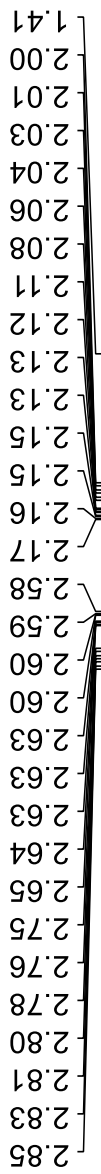

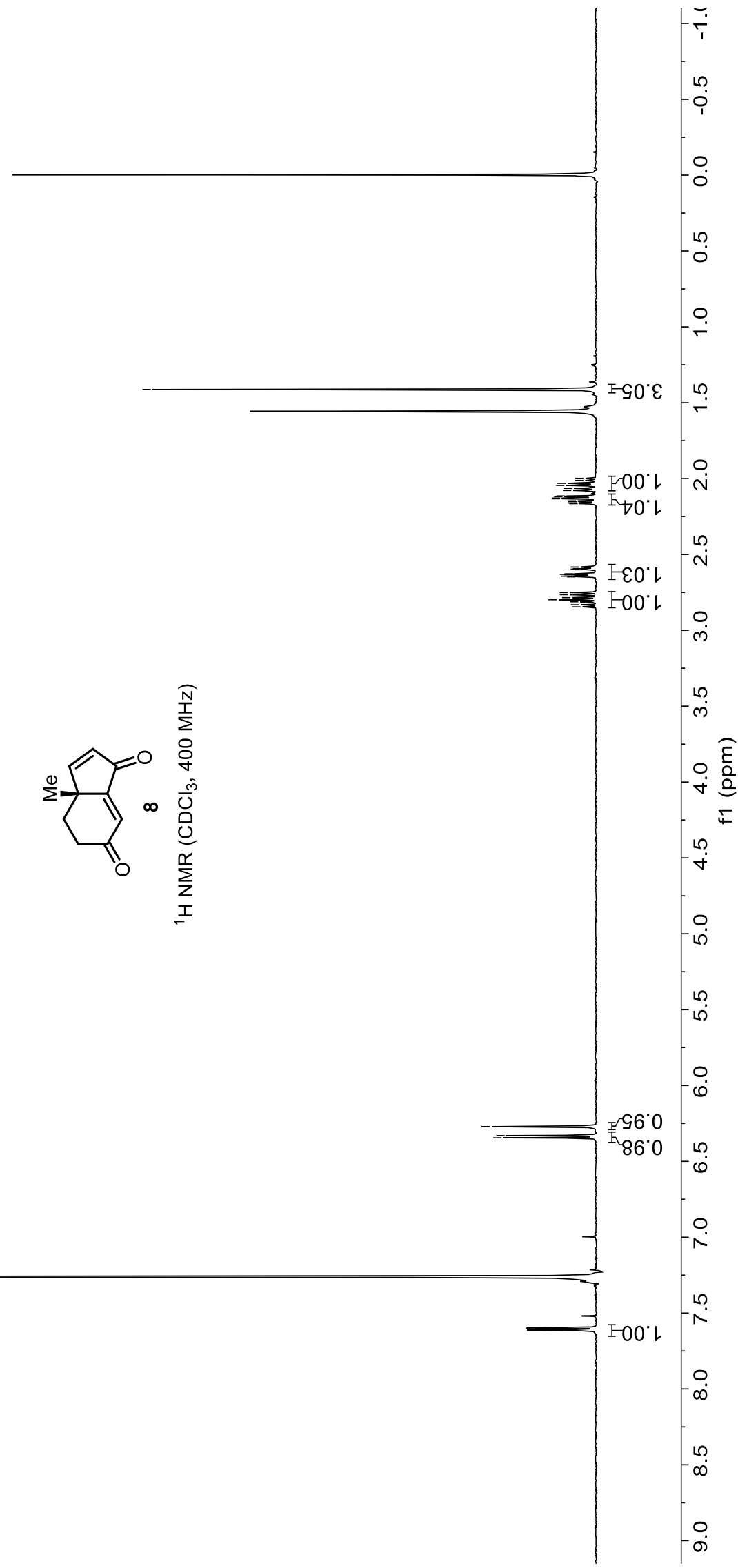




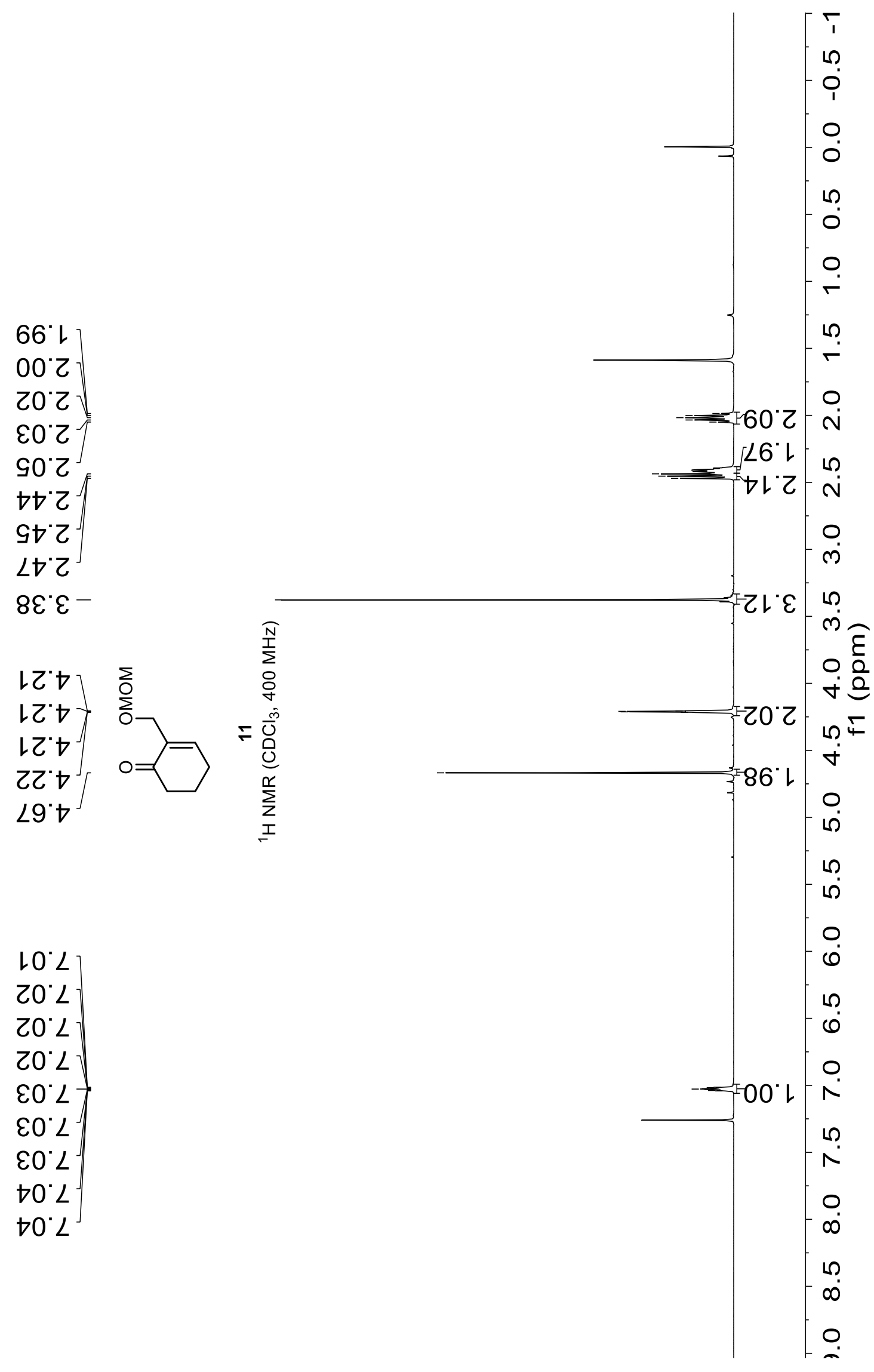




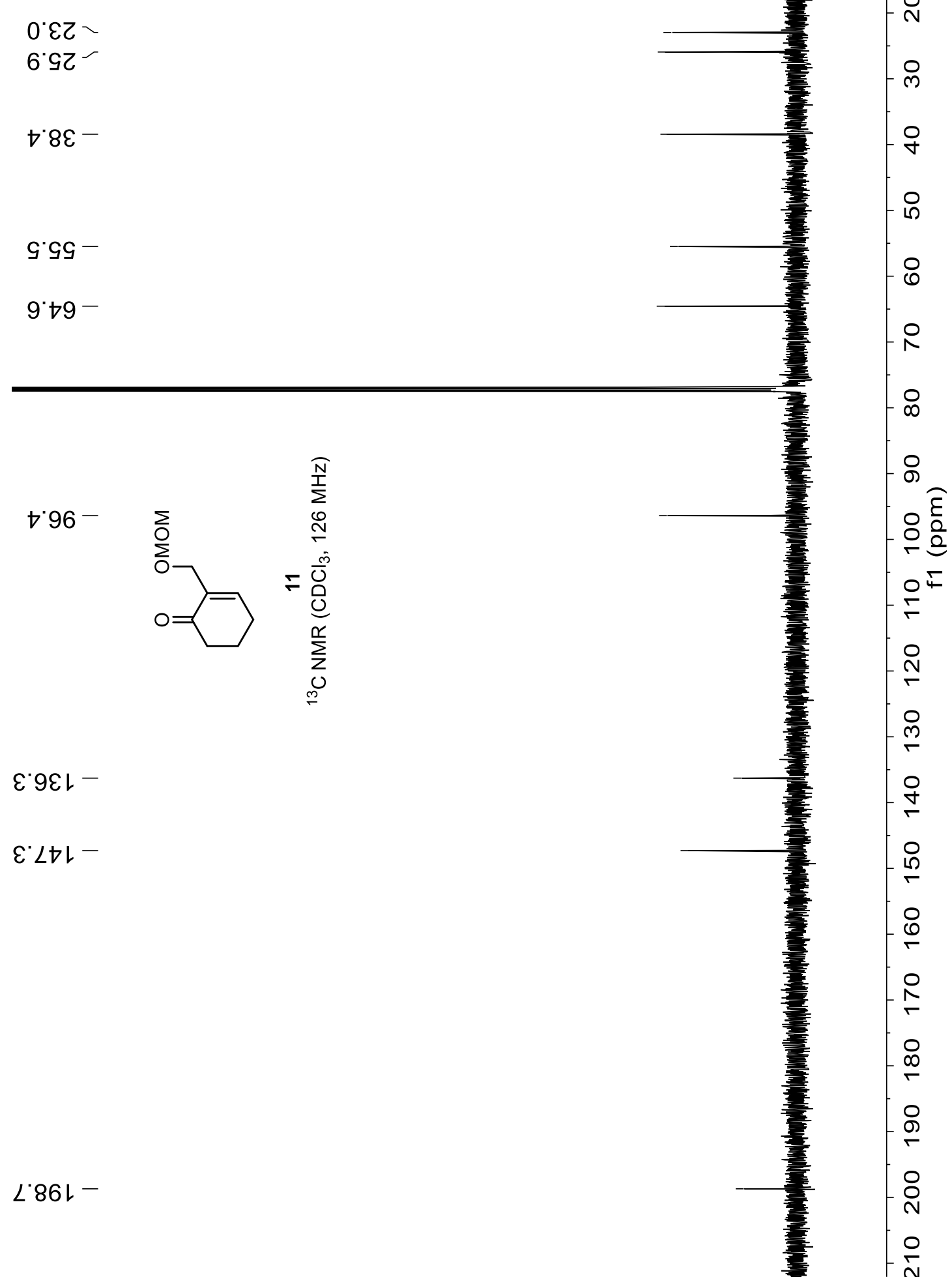




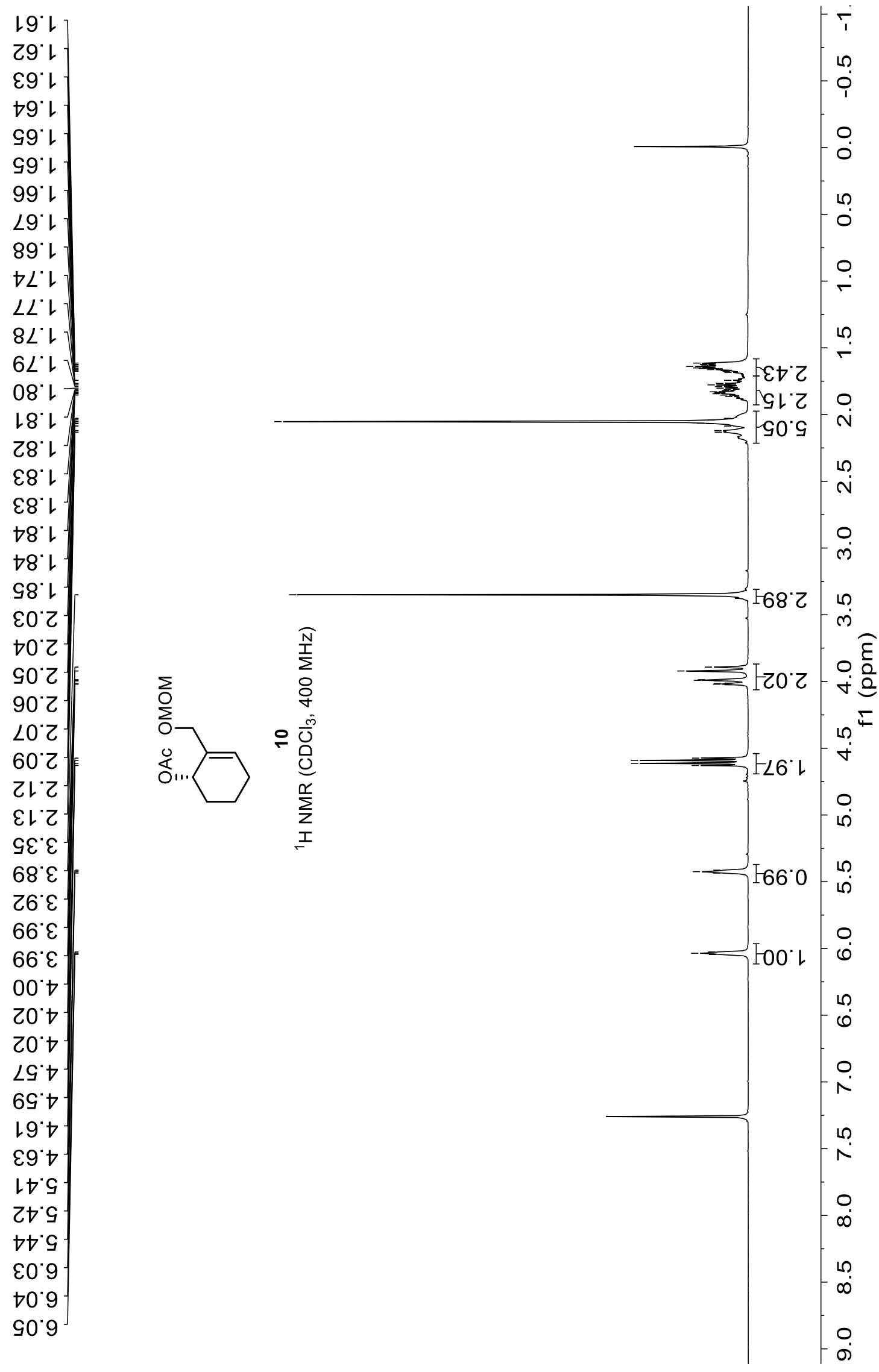




$$
\begin{aligned}
& 0.8 L> \\
& \text { S.L- } \\
& \text { LG乙- } \\
& L^{\circ} 8 Z^{-}
\end{aligned}
$$$$
\text { t) }
$$$$
\text { Z. } \angle 9>
$$$$
6.89^{\prime}
$$$$
6.96-\quad \sum_{0}
$$

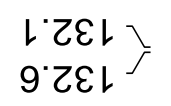

6.0LL-

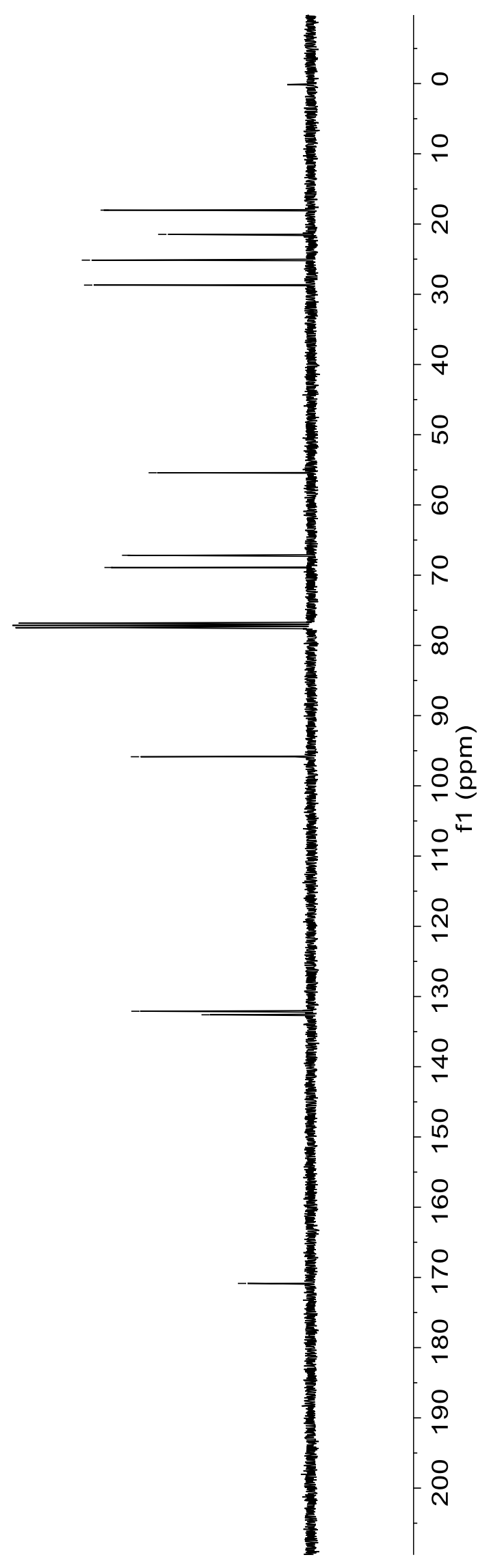



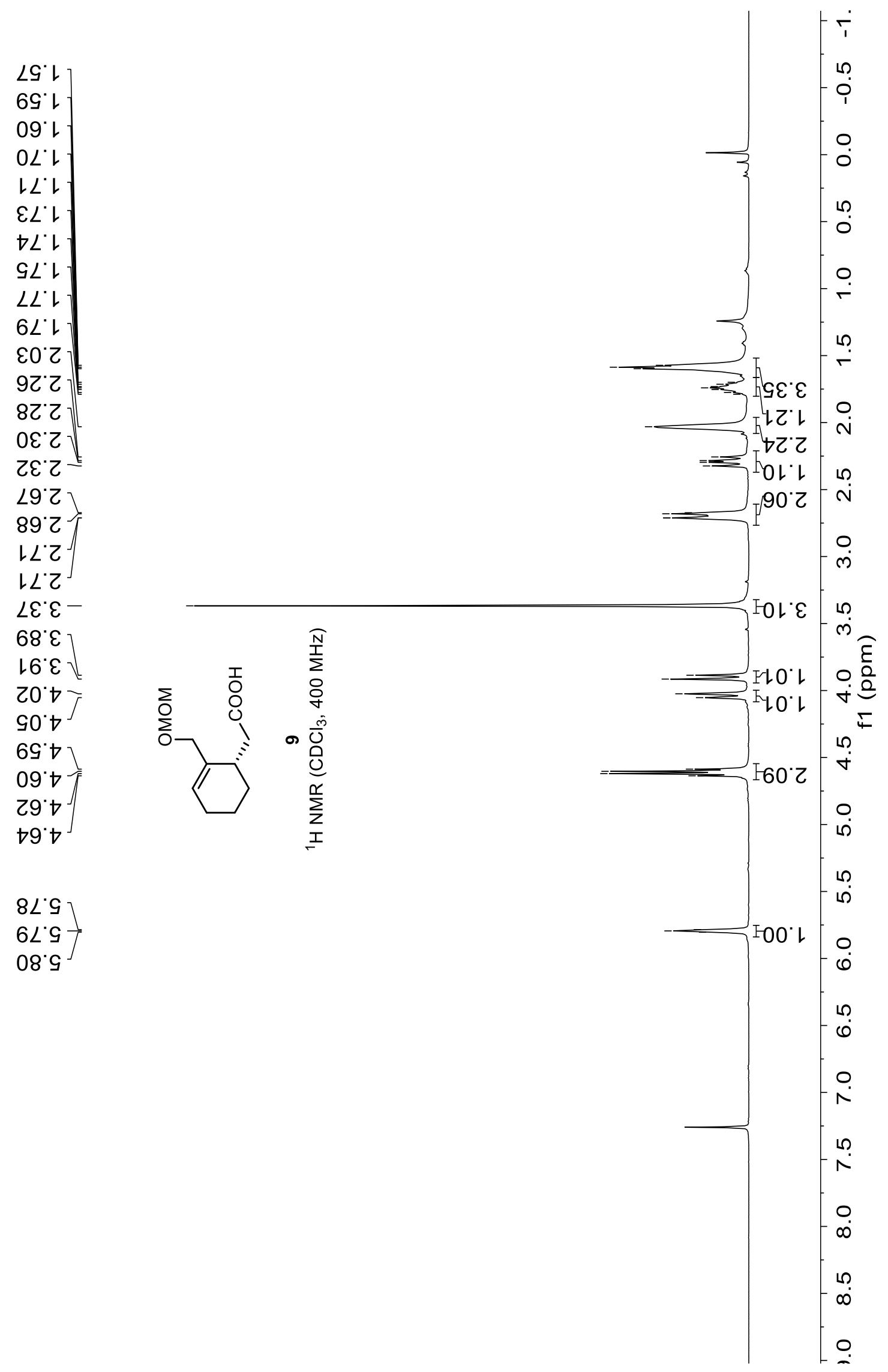

$\left.\begin{array}{l}8 L G \\ 6 L 9 \\ 08 \cdot 9\end{array}\right]$ 

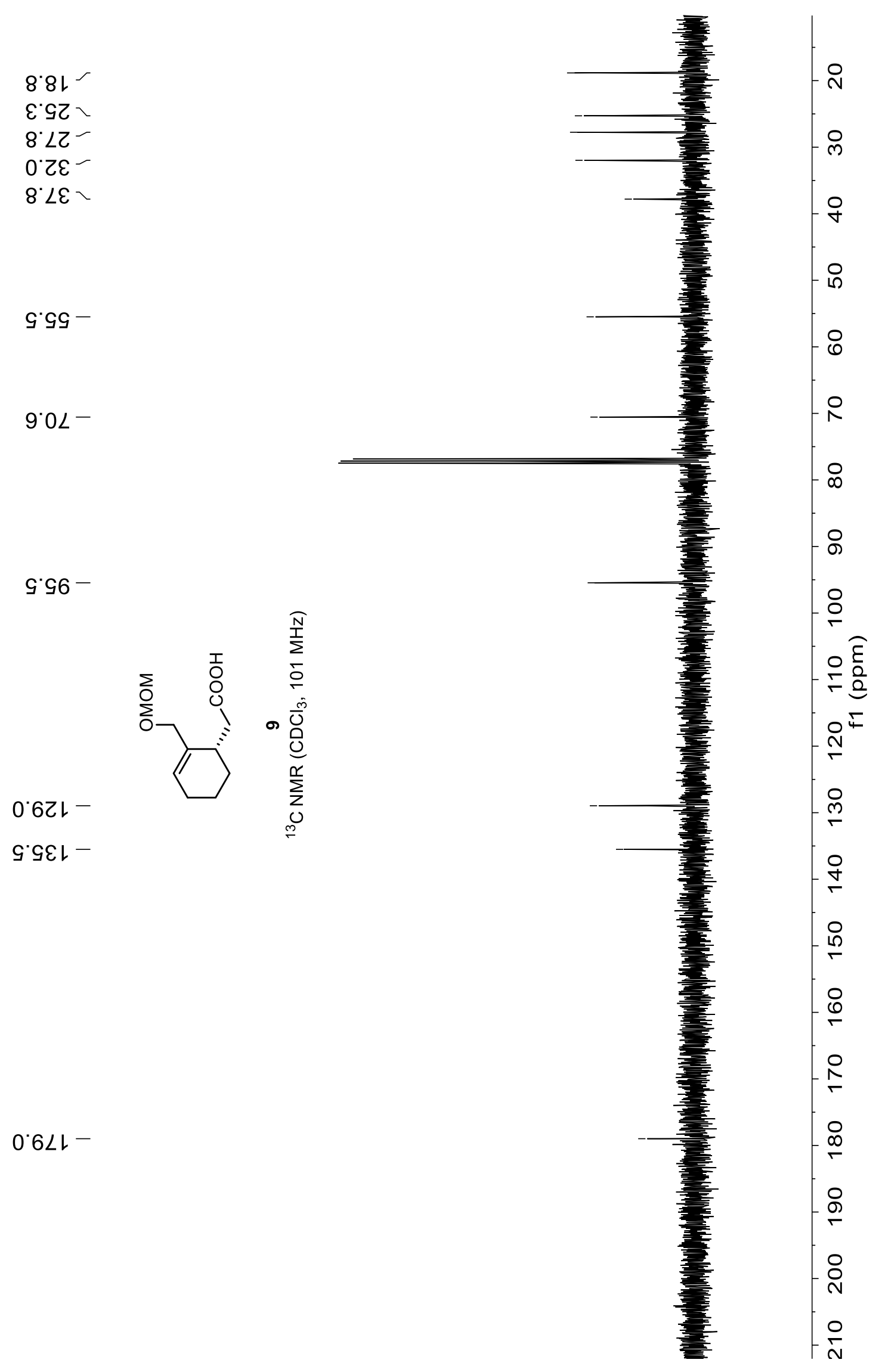


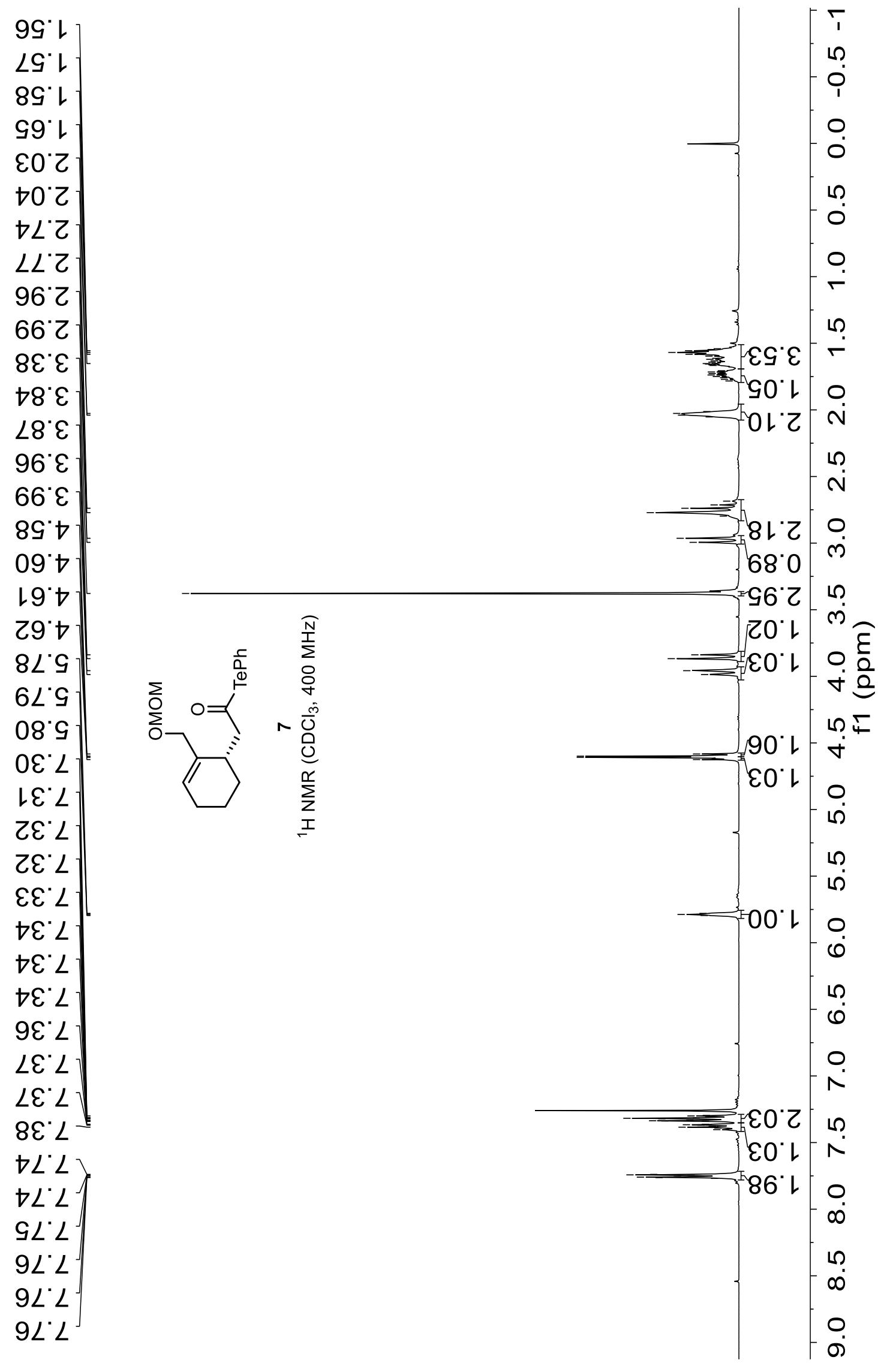




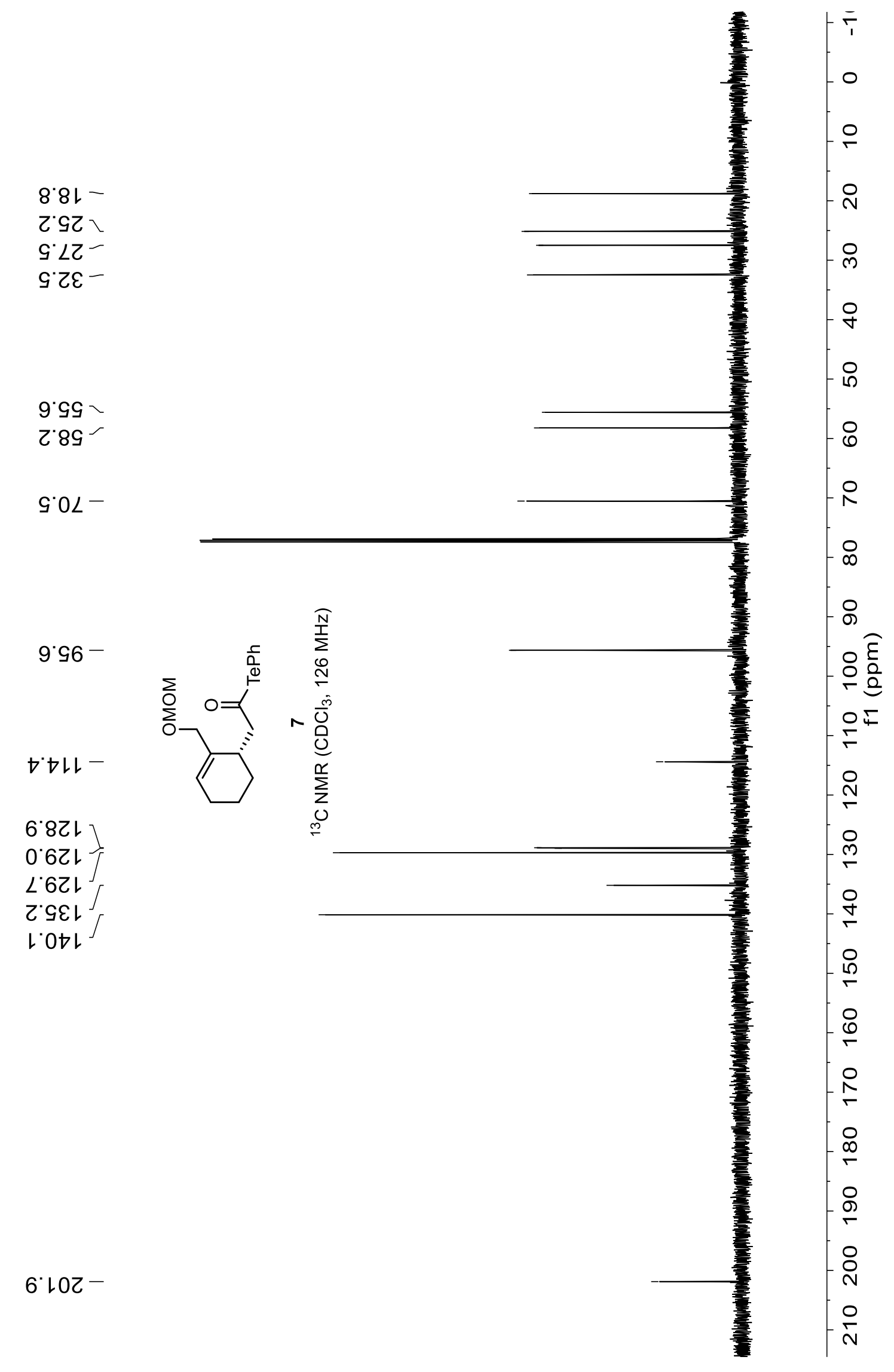



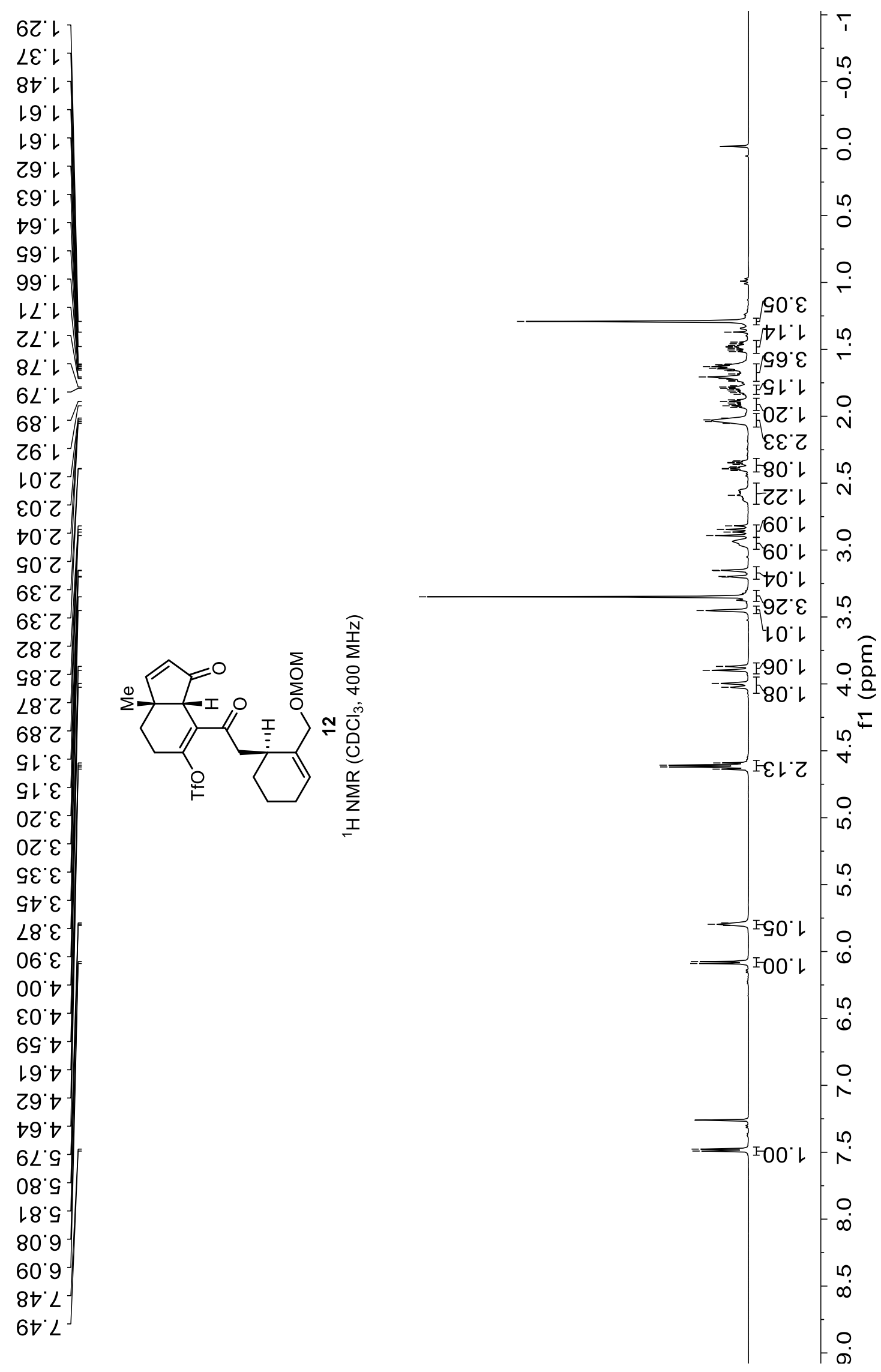


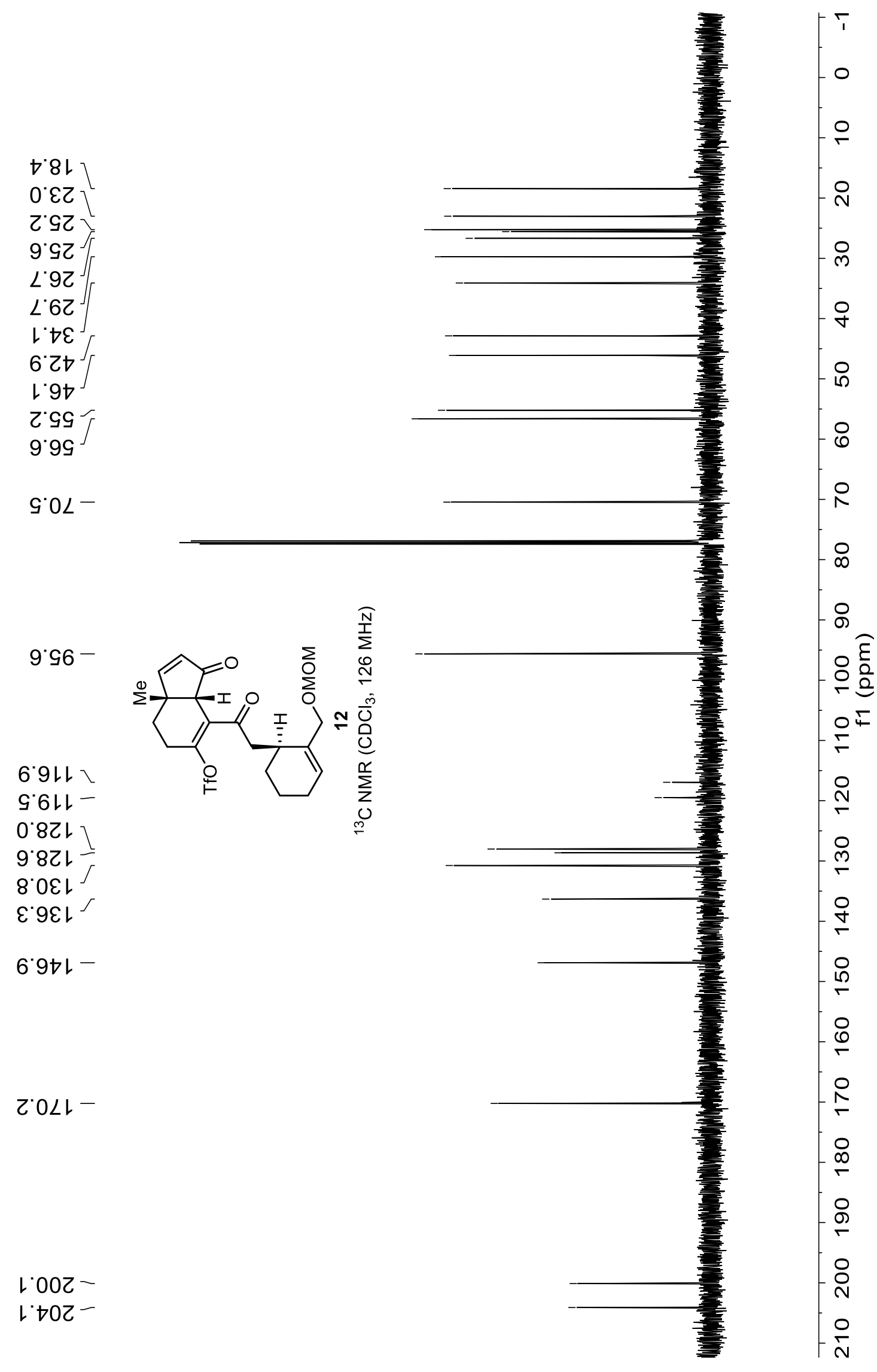




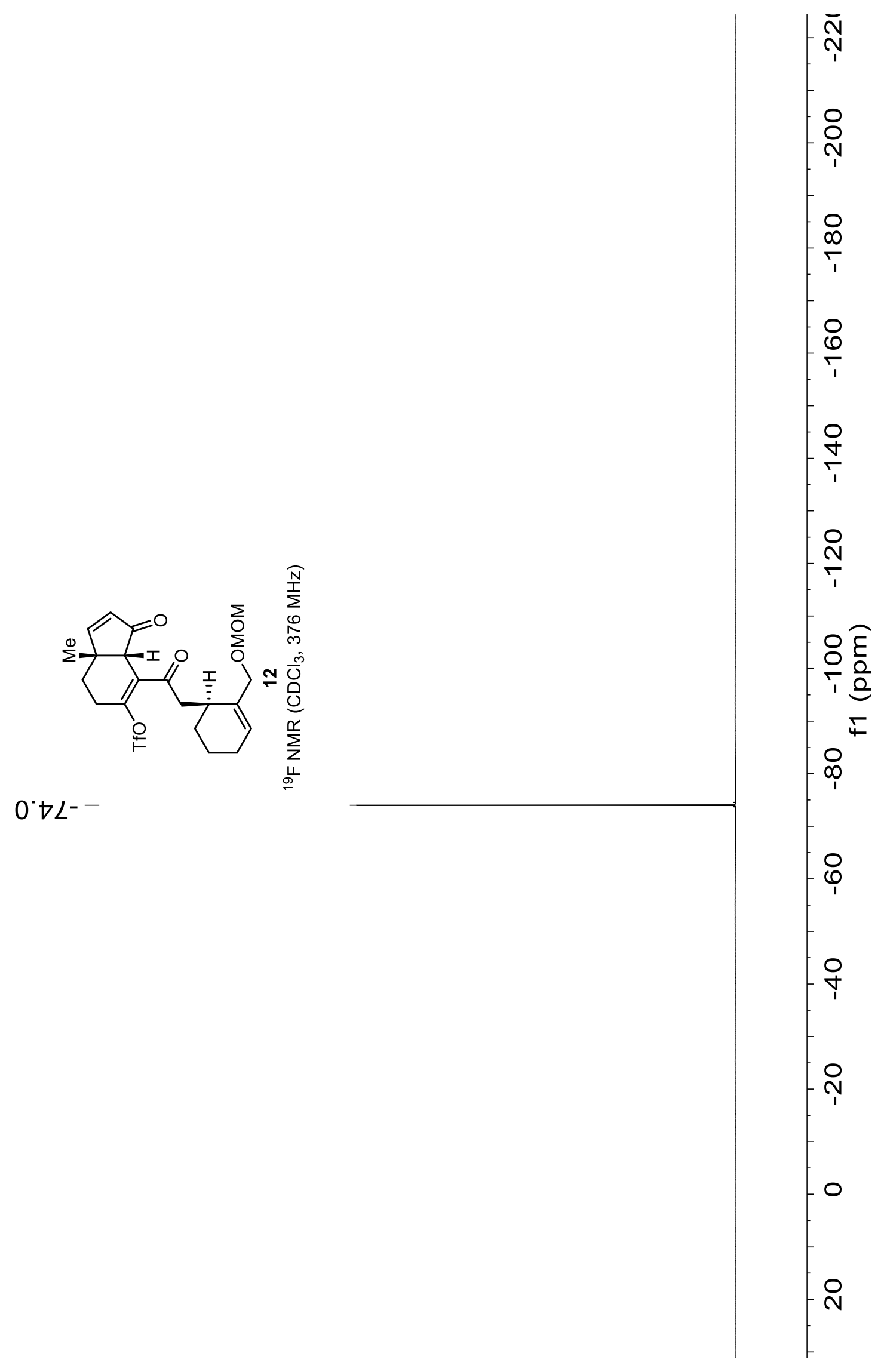




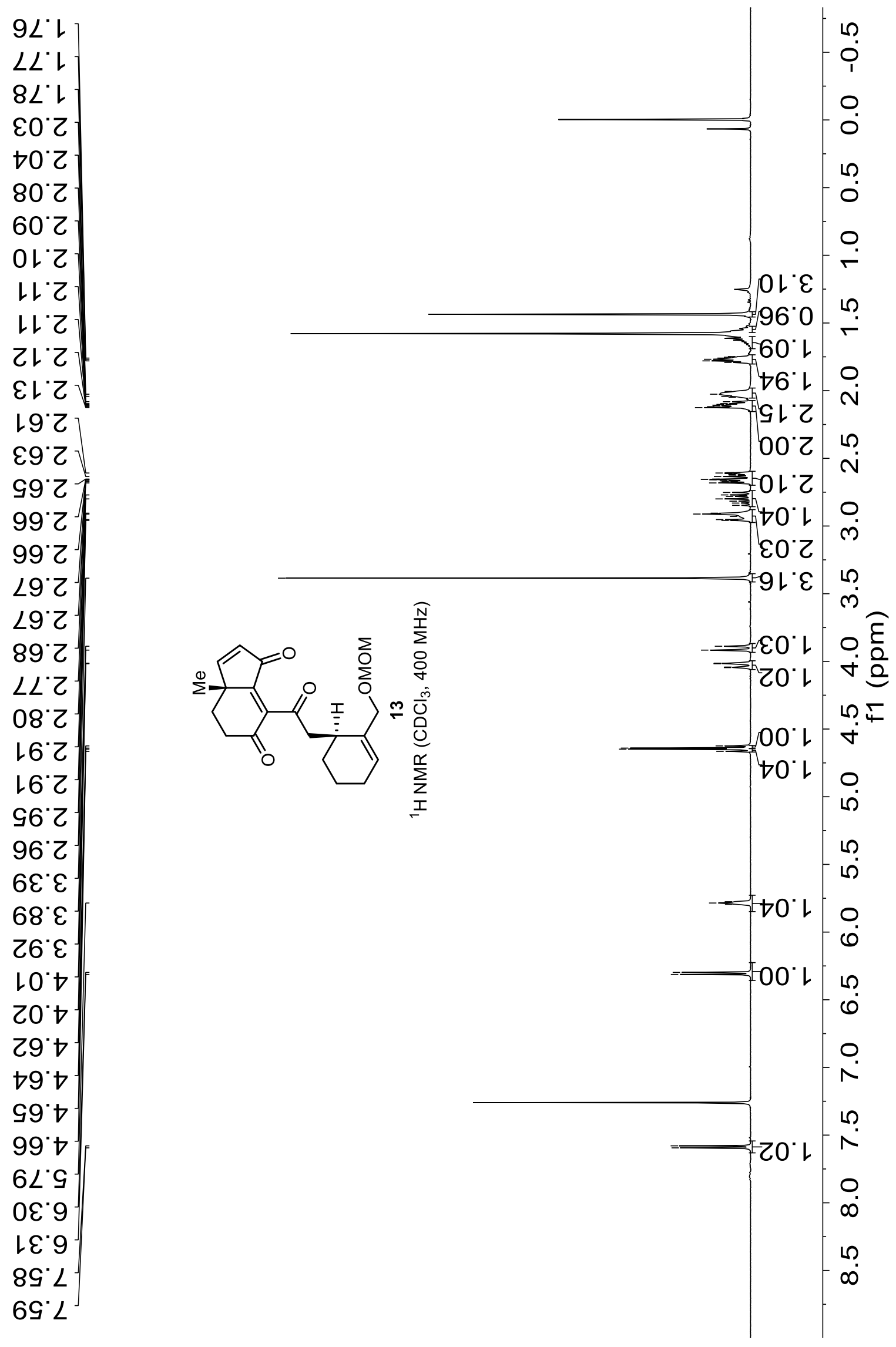




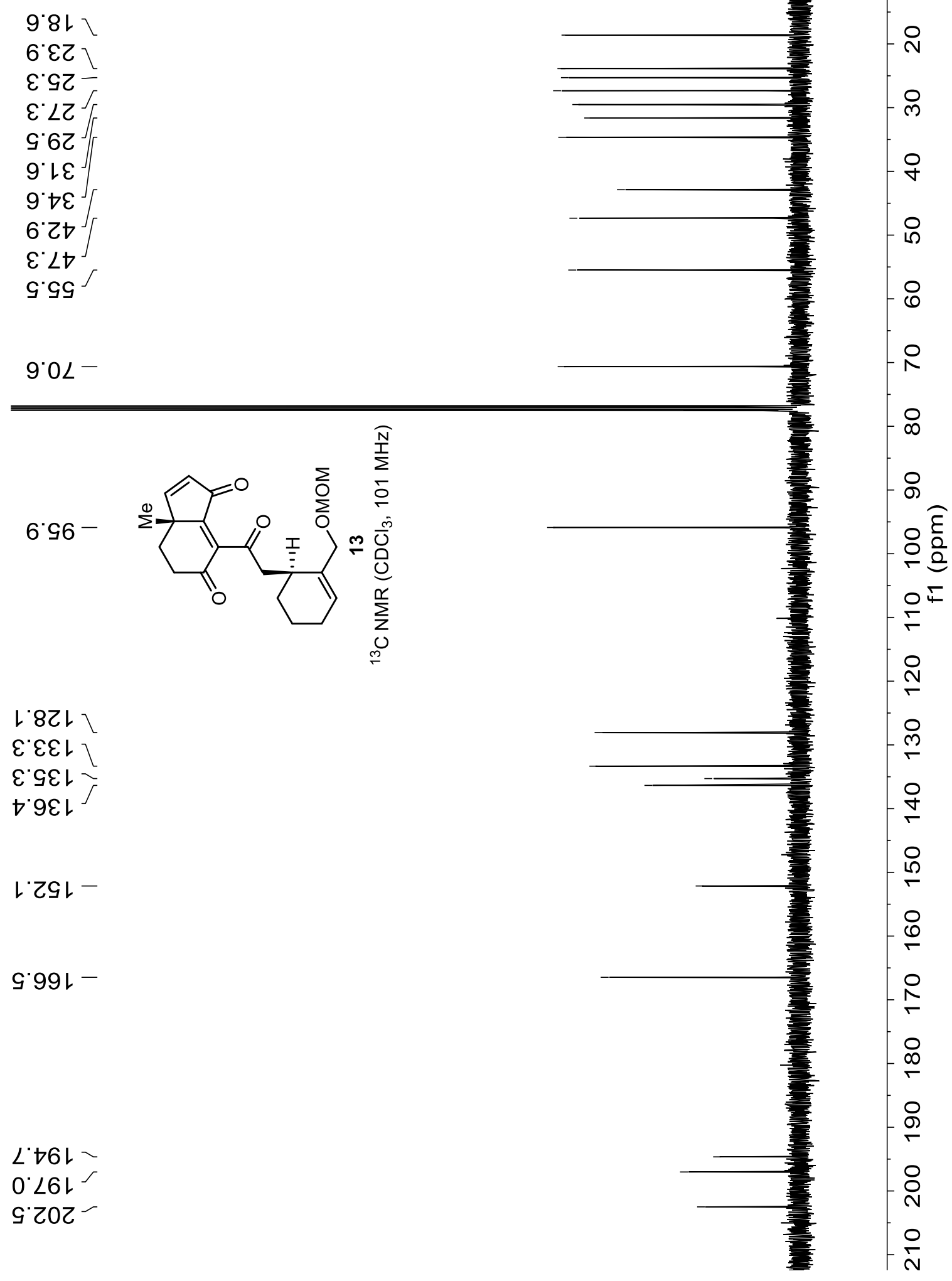




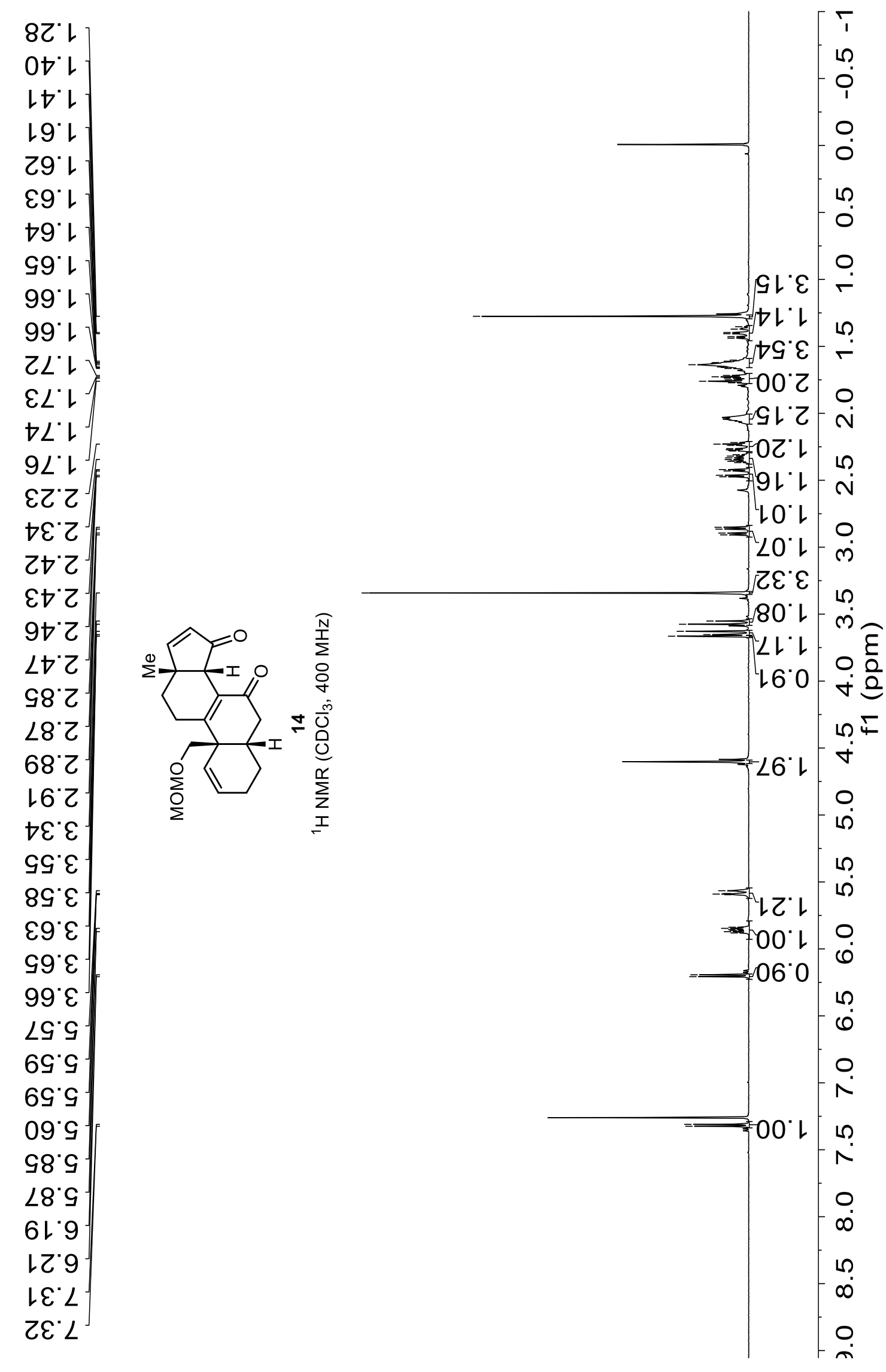




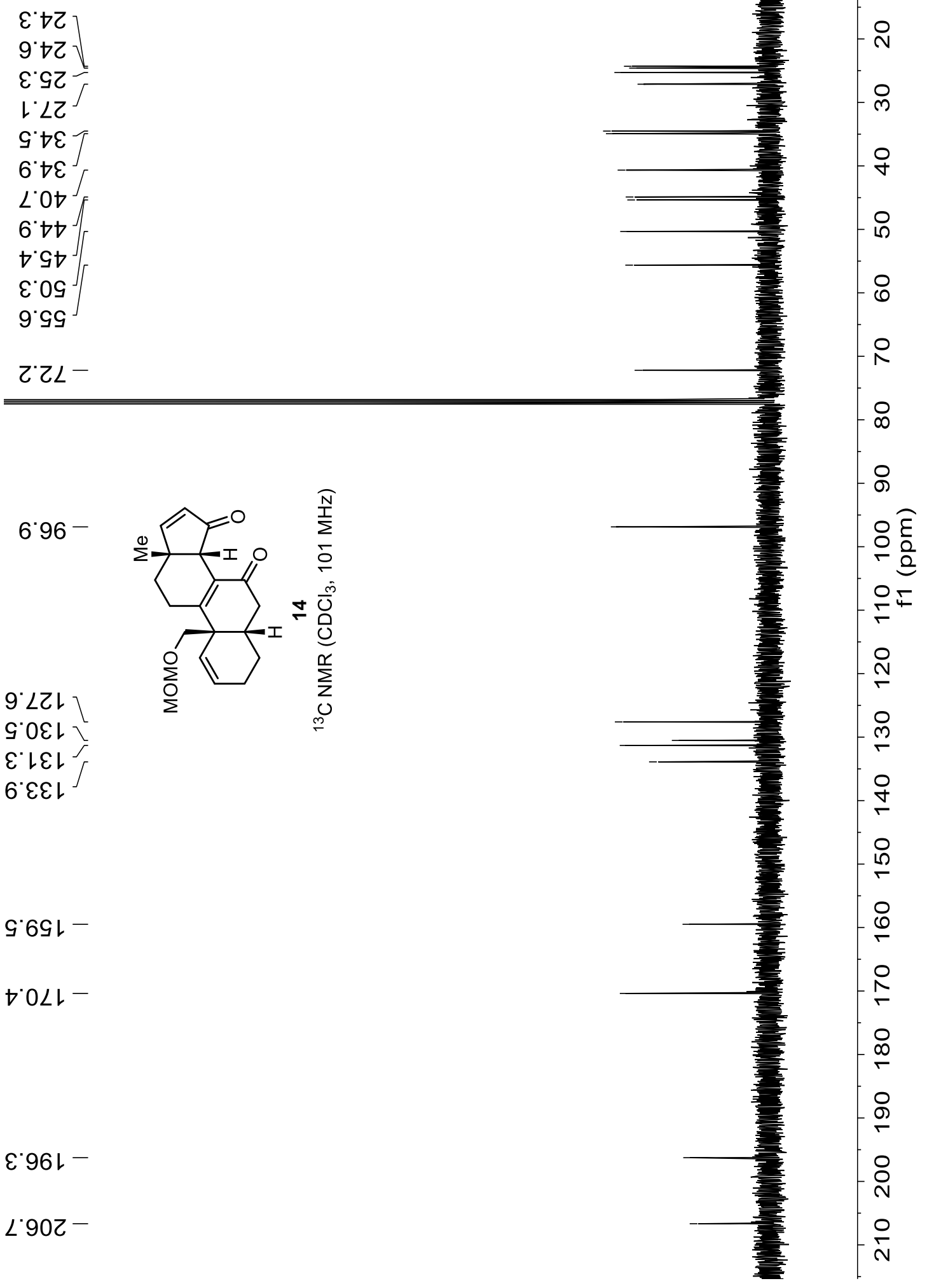




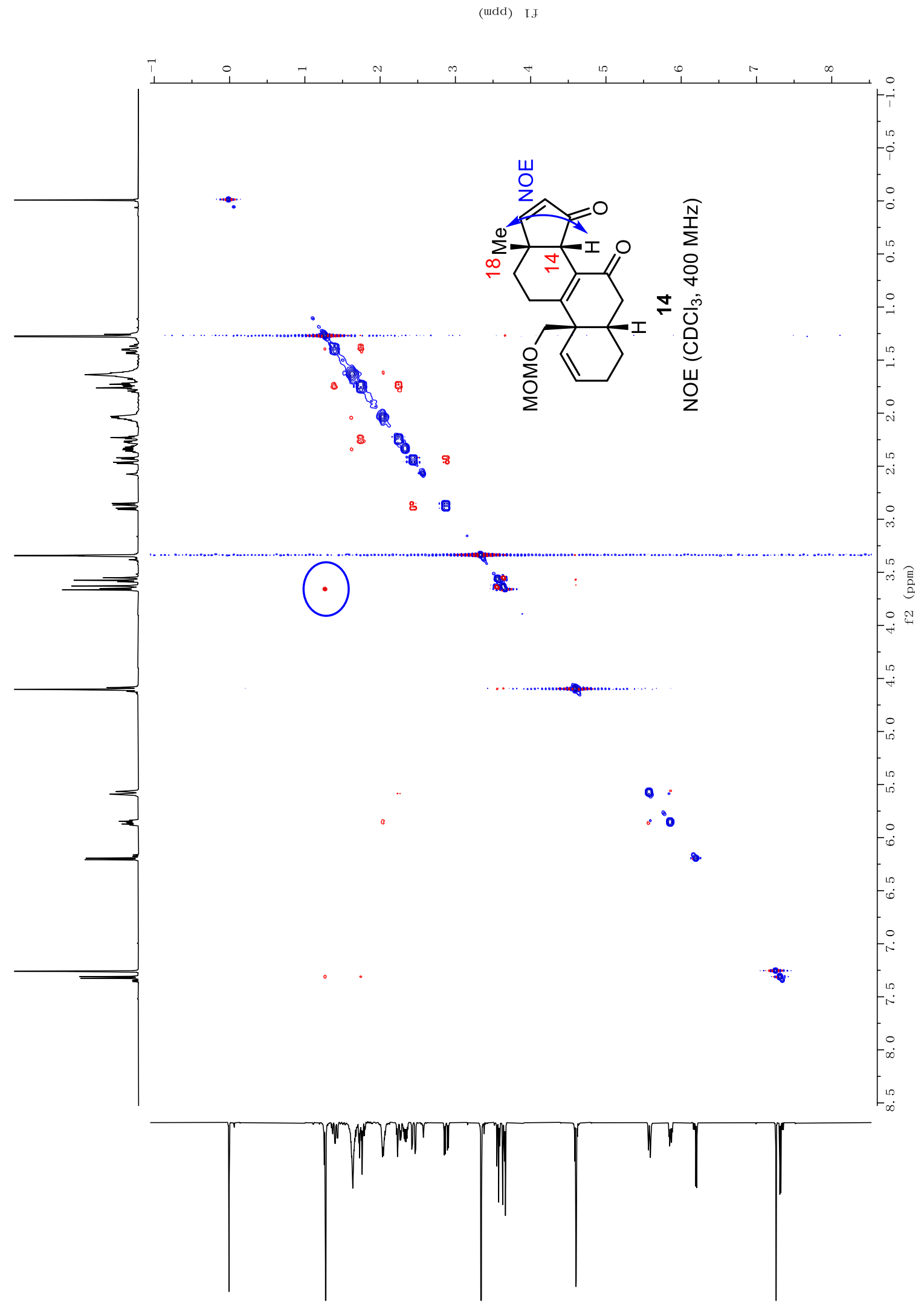




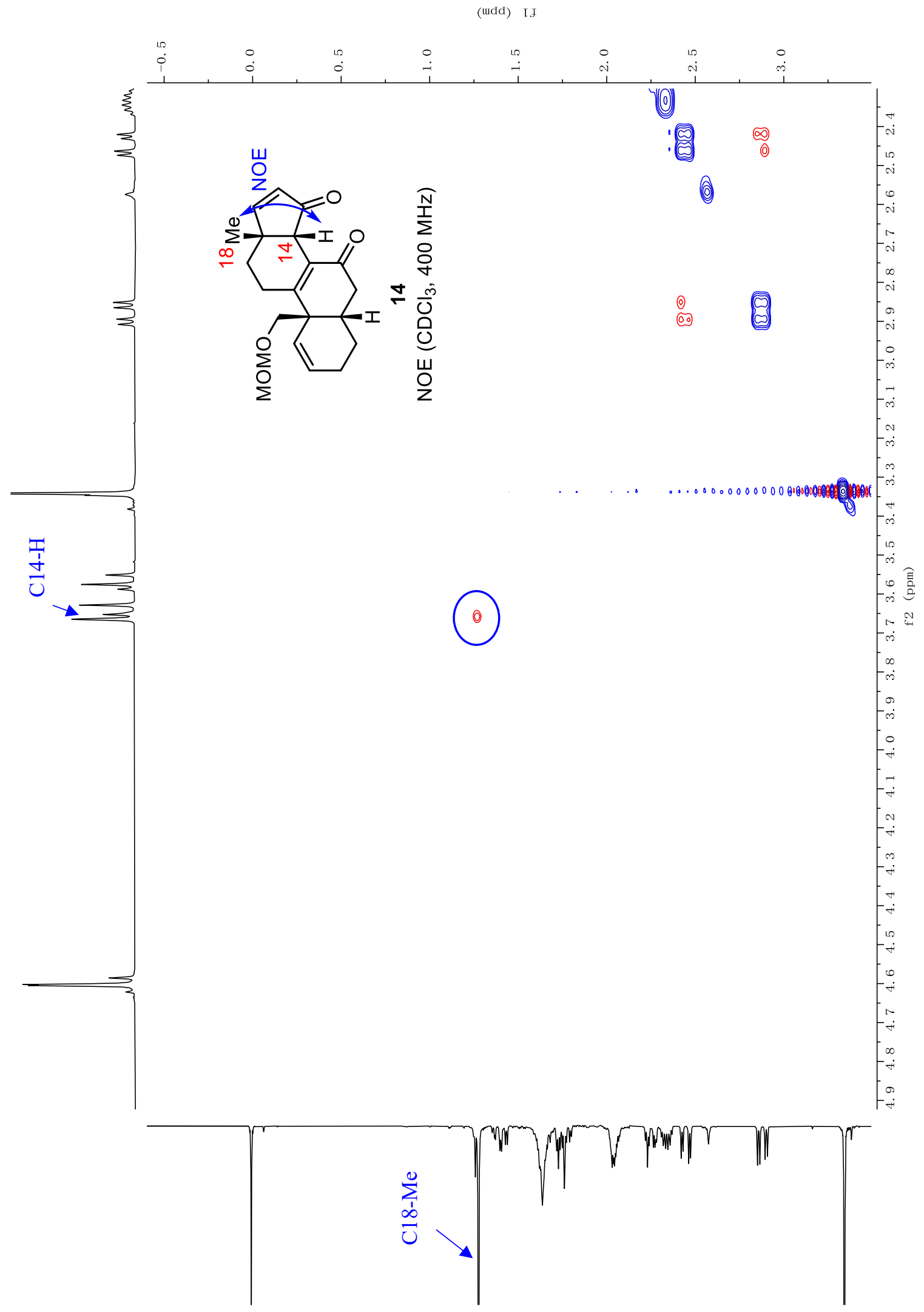



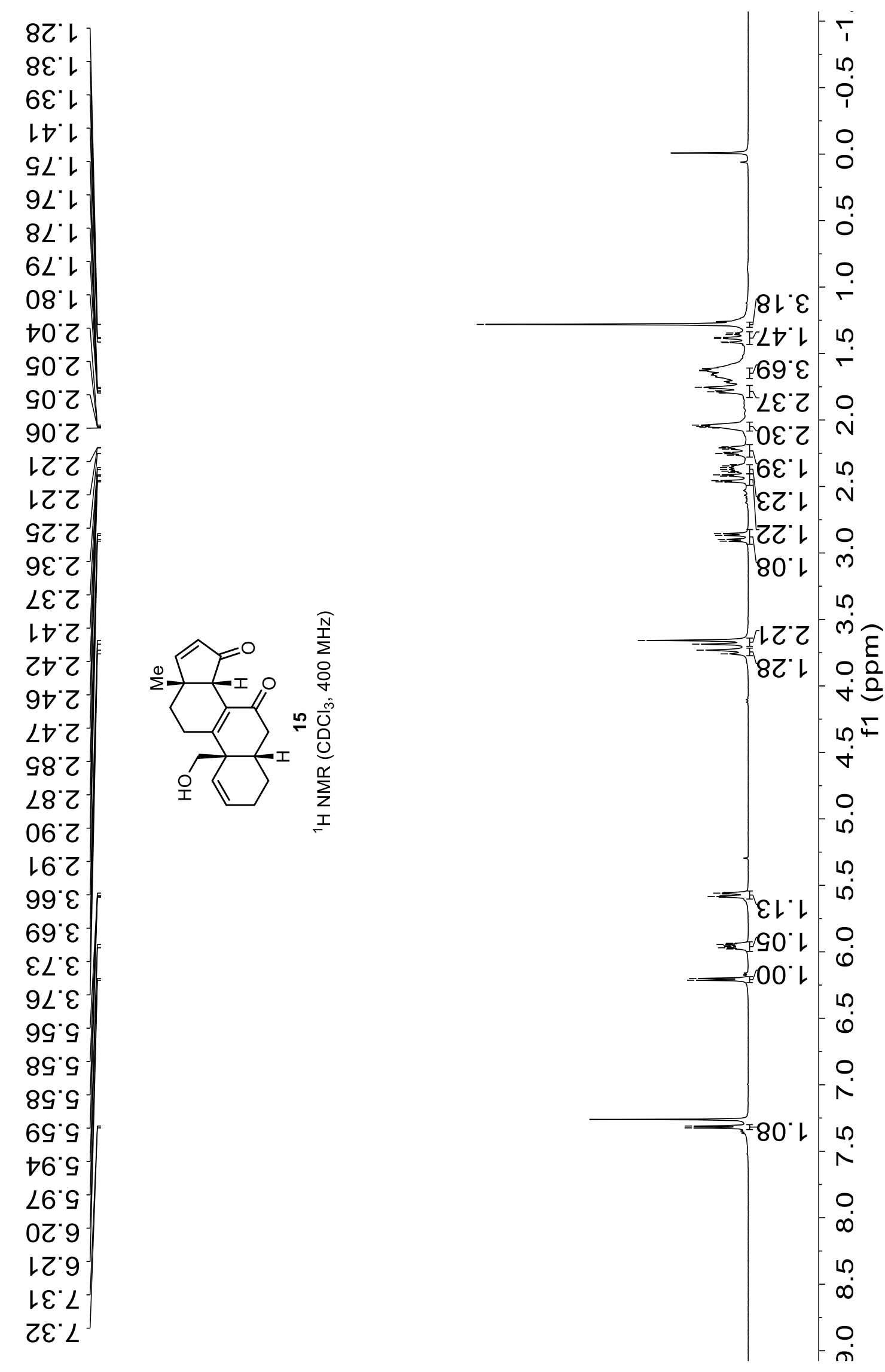

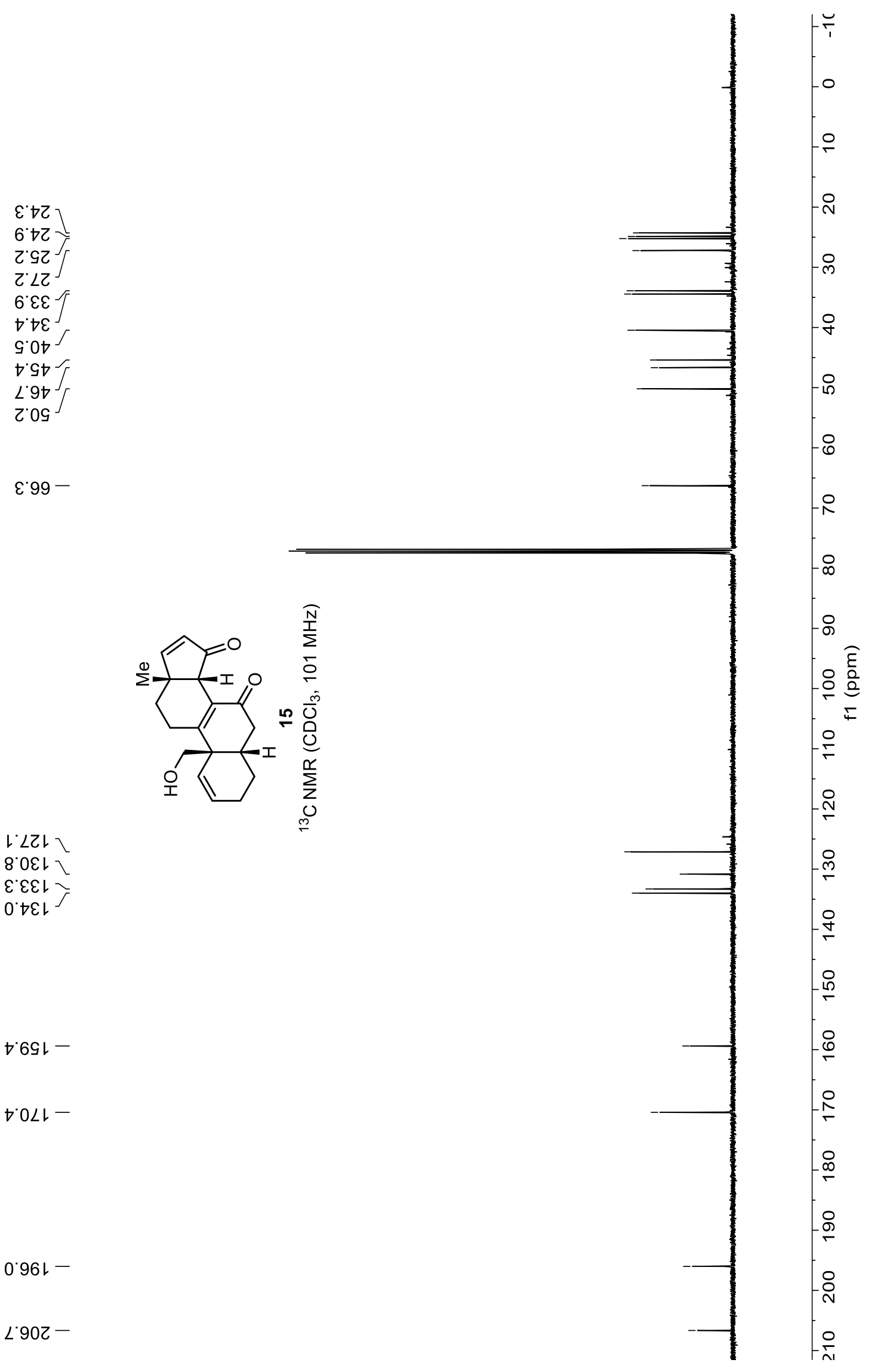

L LZL 8.0हL \ $\varepsilon \varepsilon \varepsilon \downarrow$ 厂

† $6 S$ †OLL 0.96lL'90Z- 







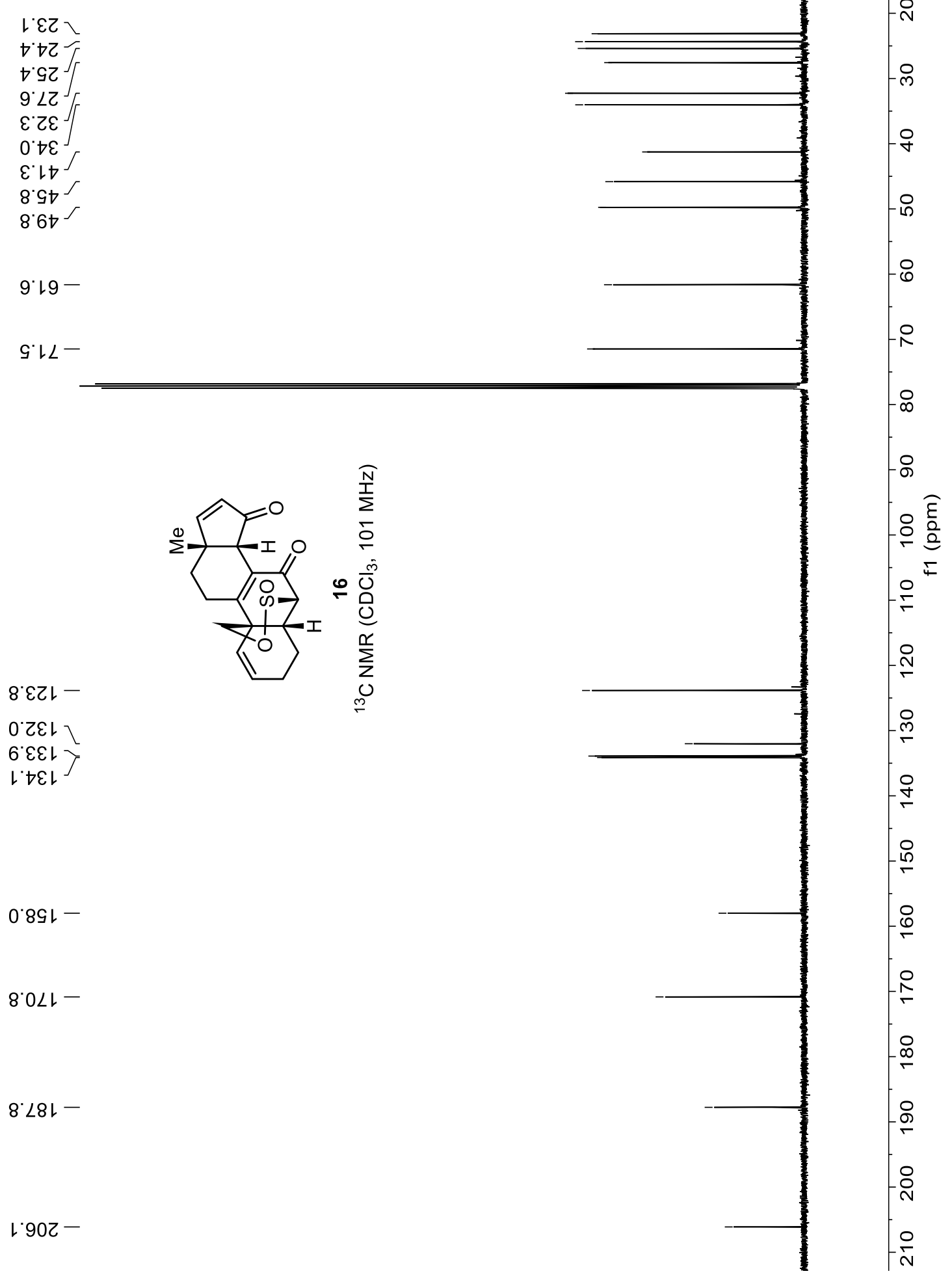




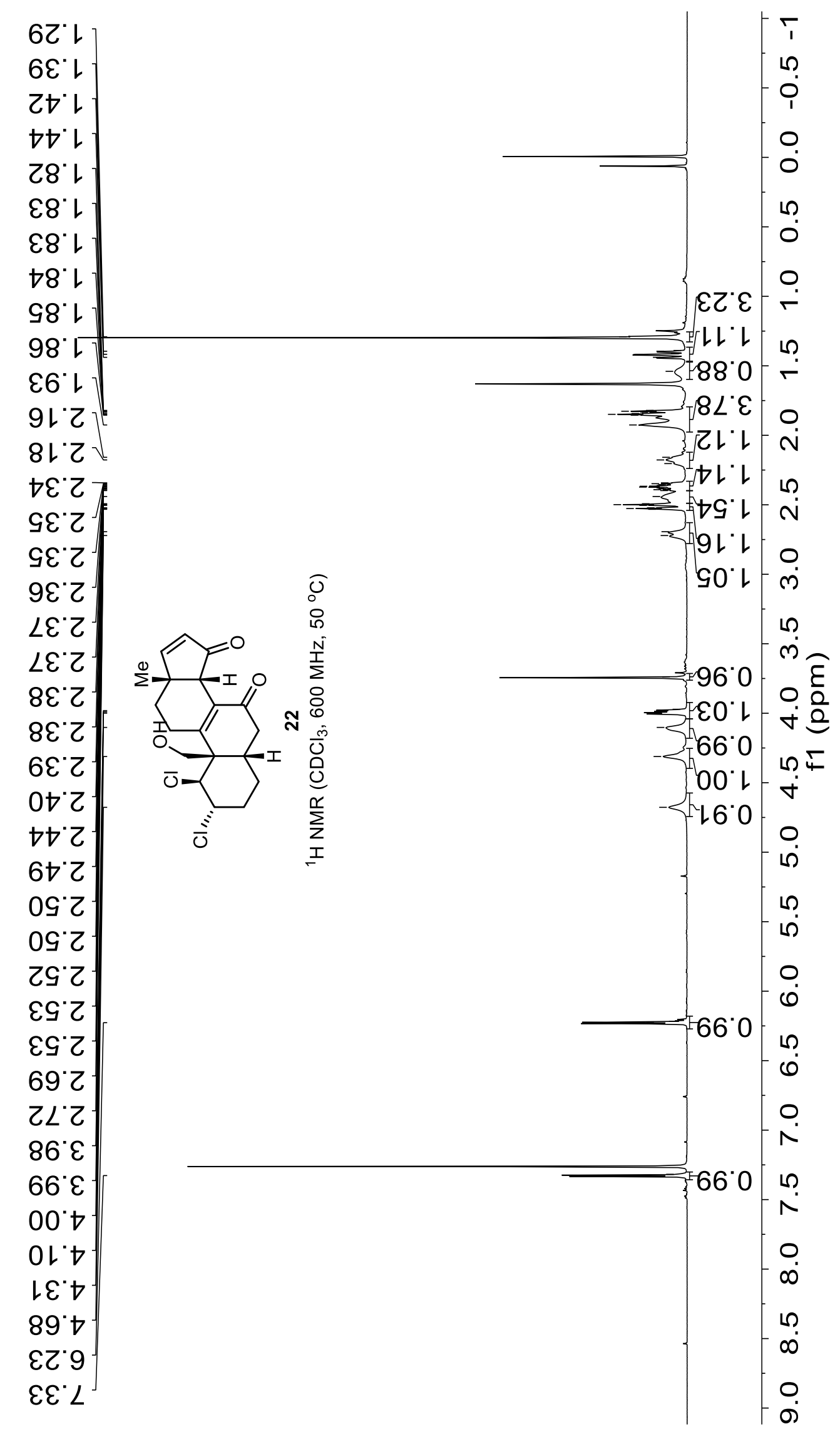




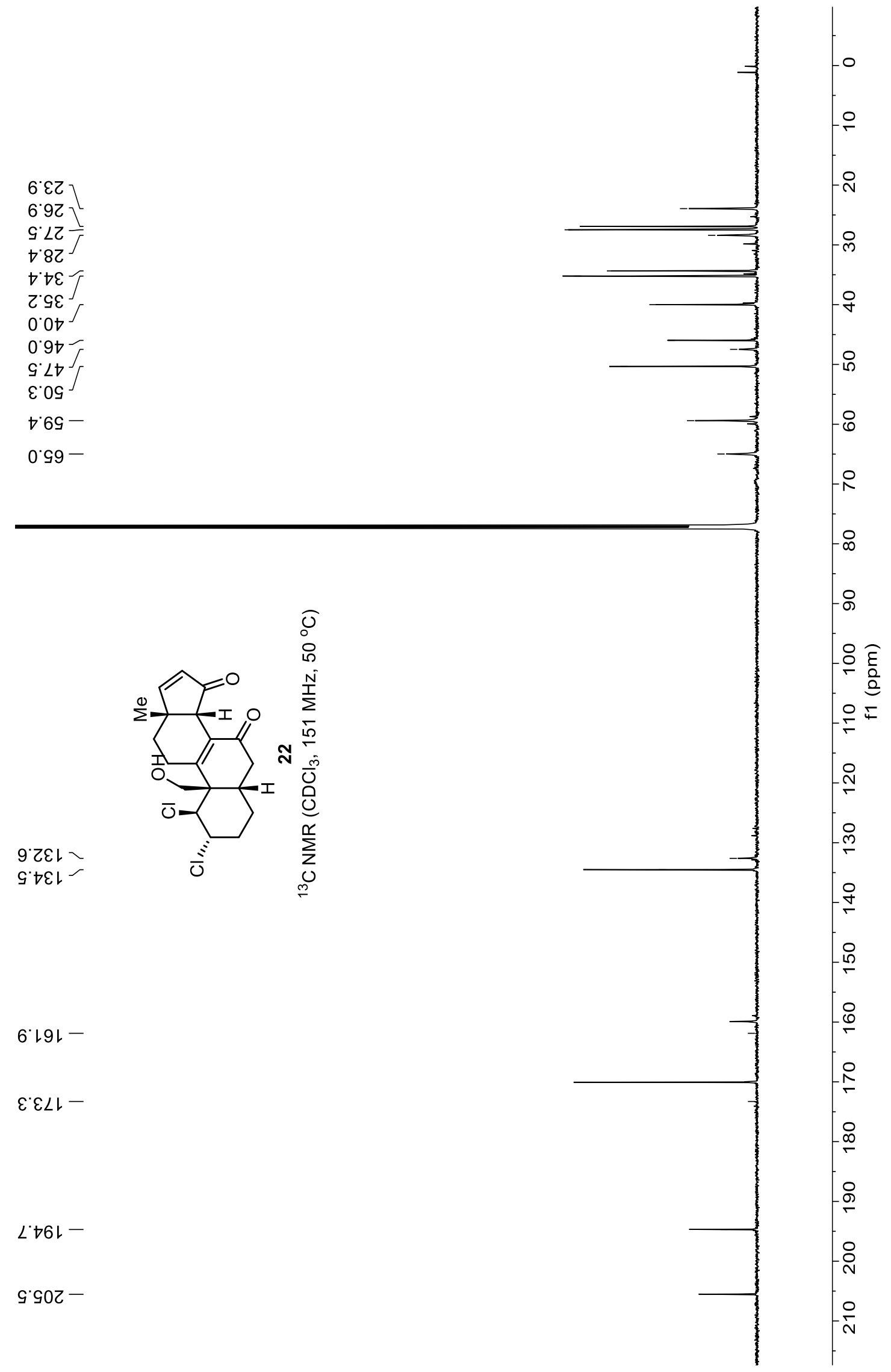




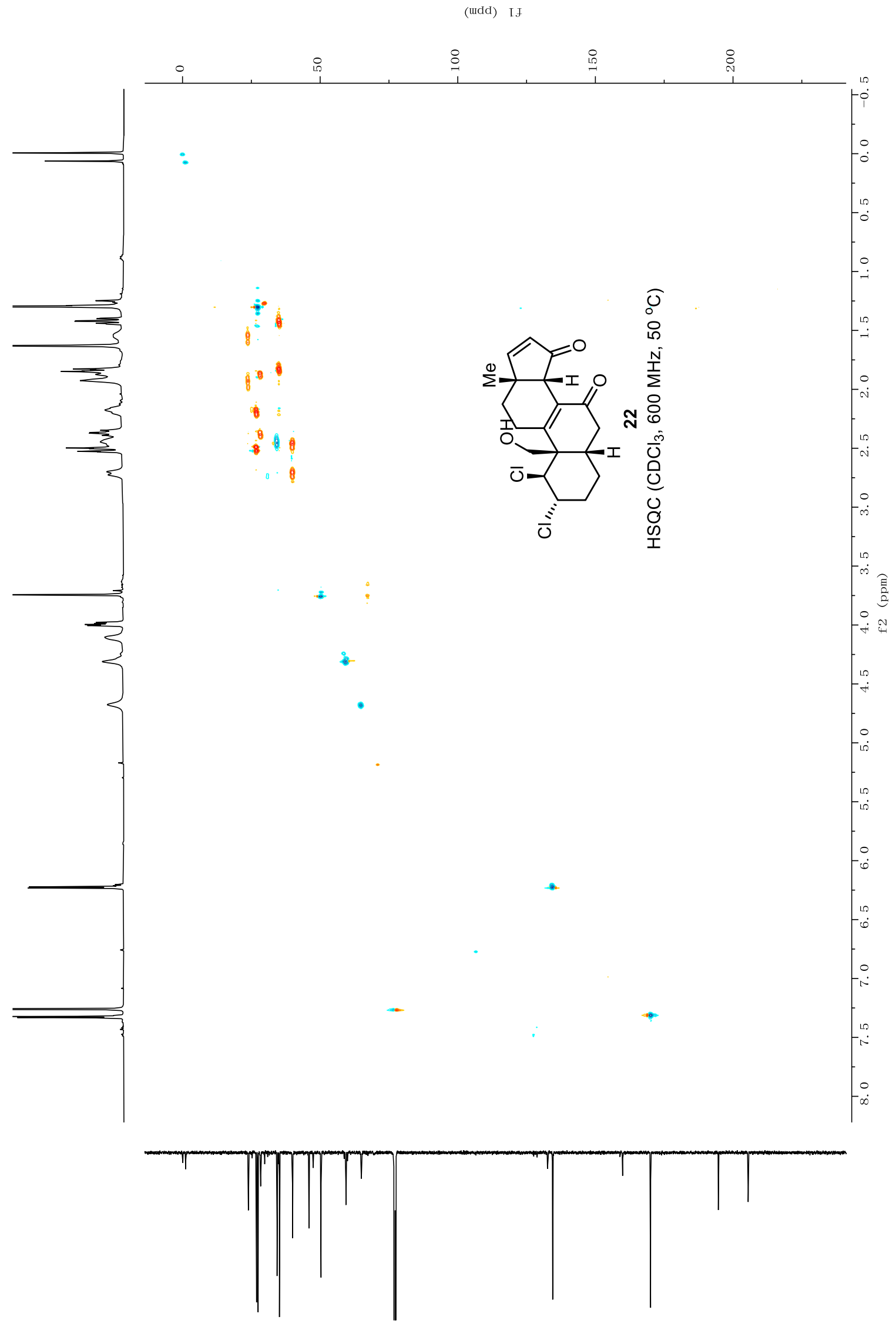




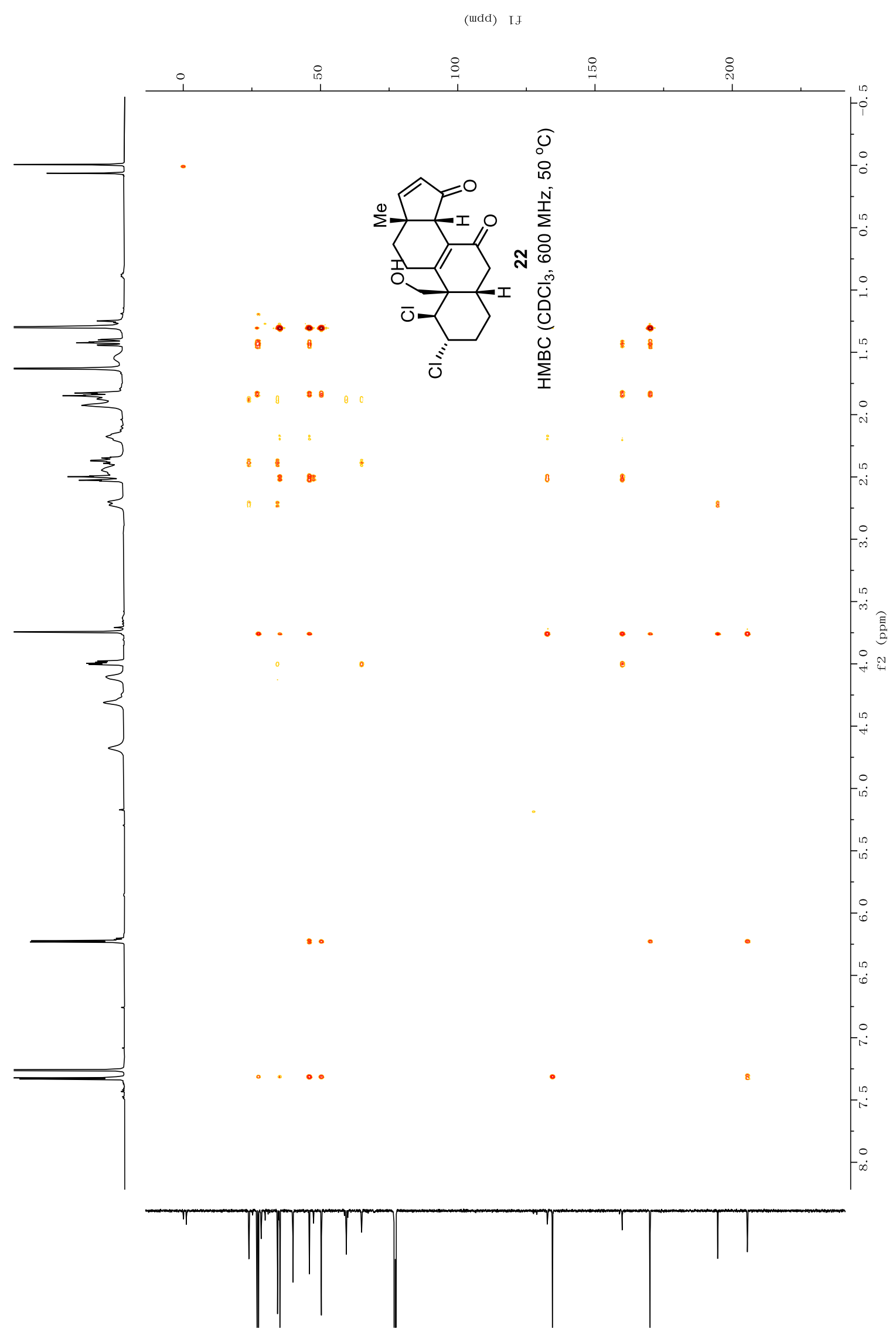




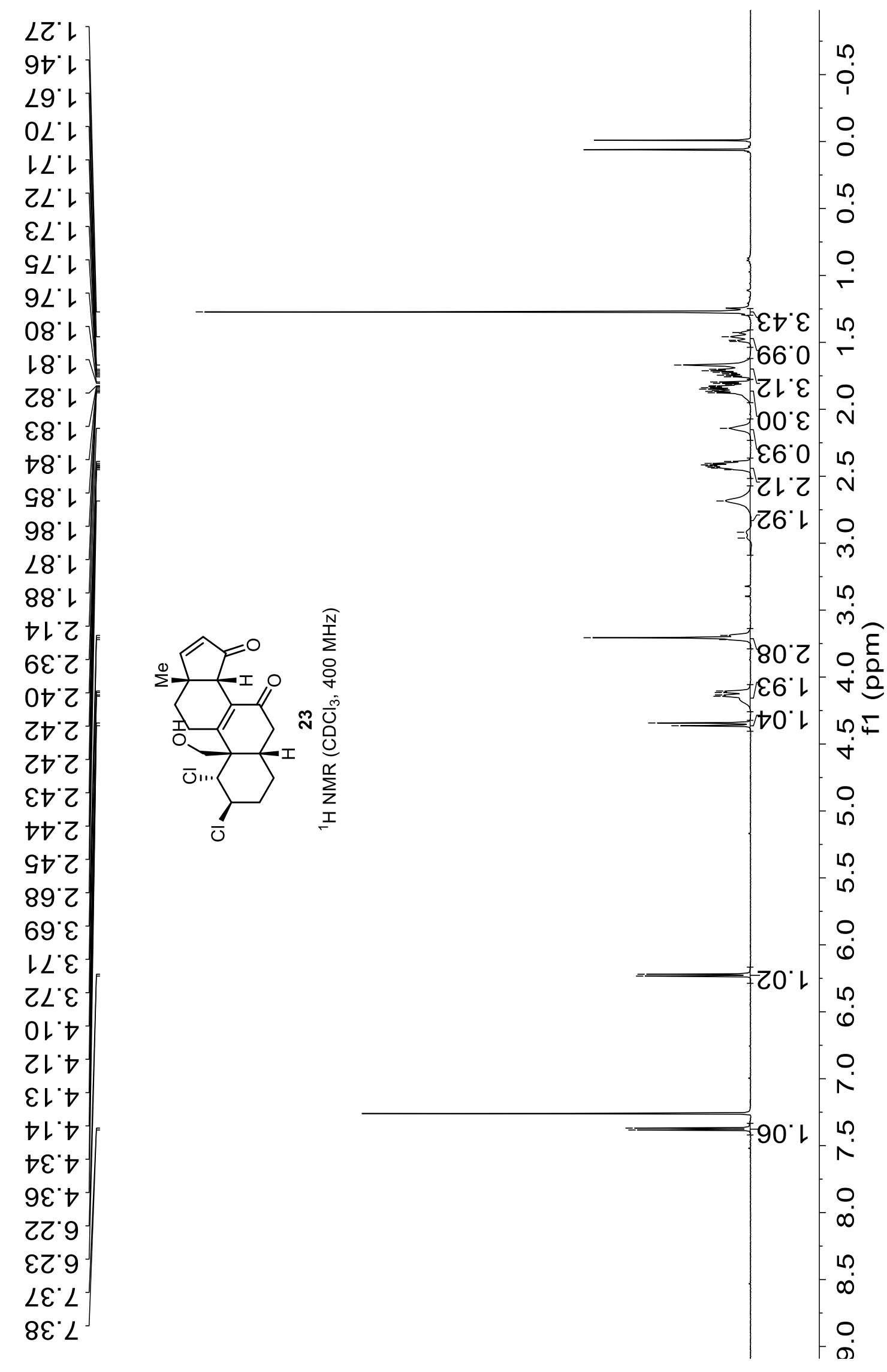




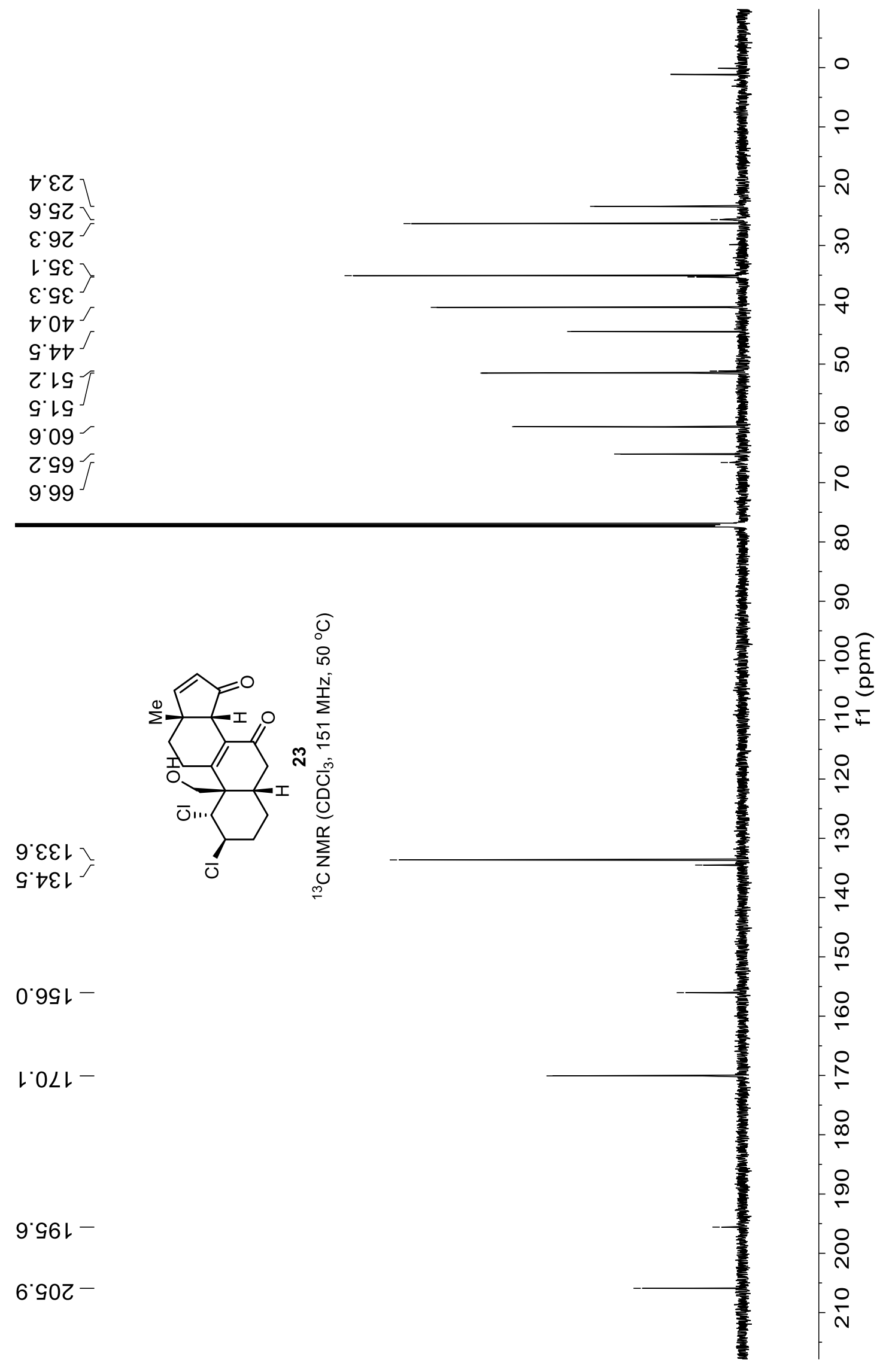


(wdd) IJ

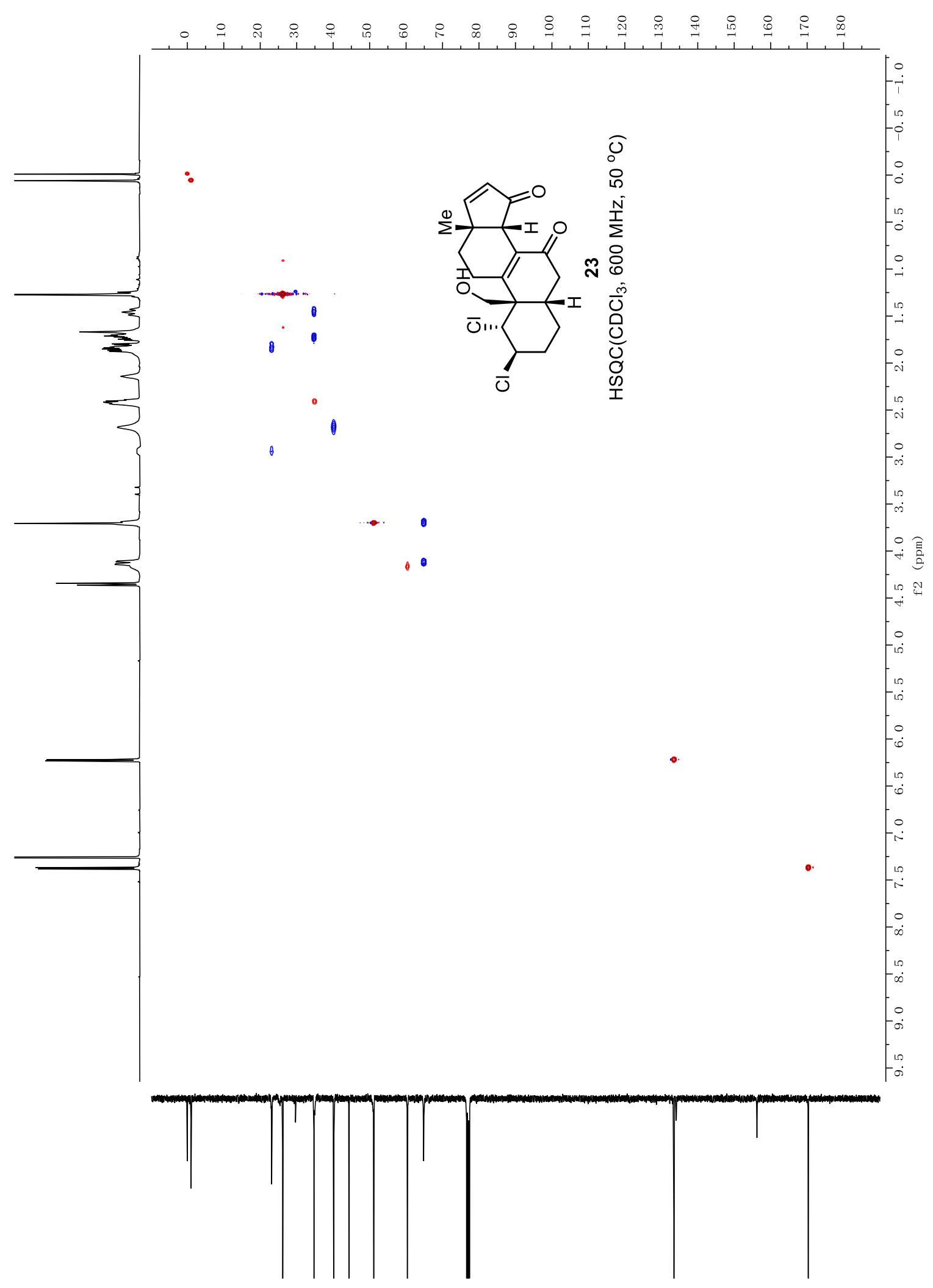




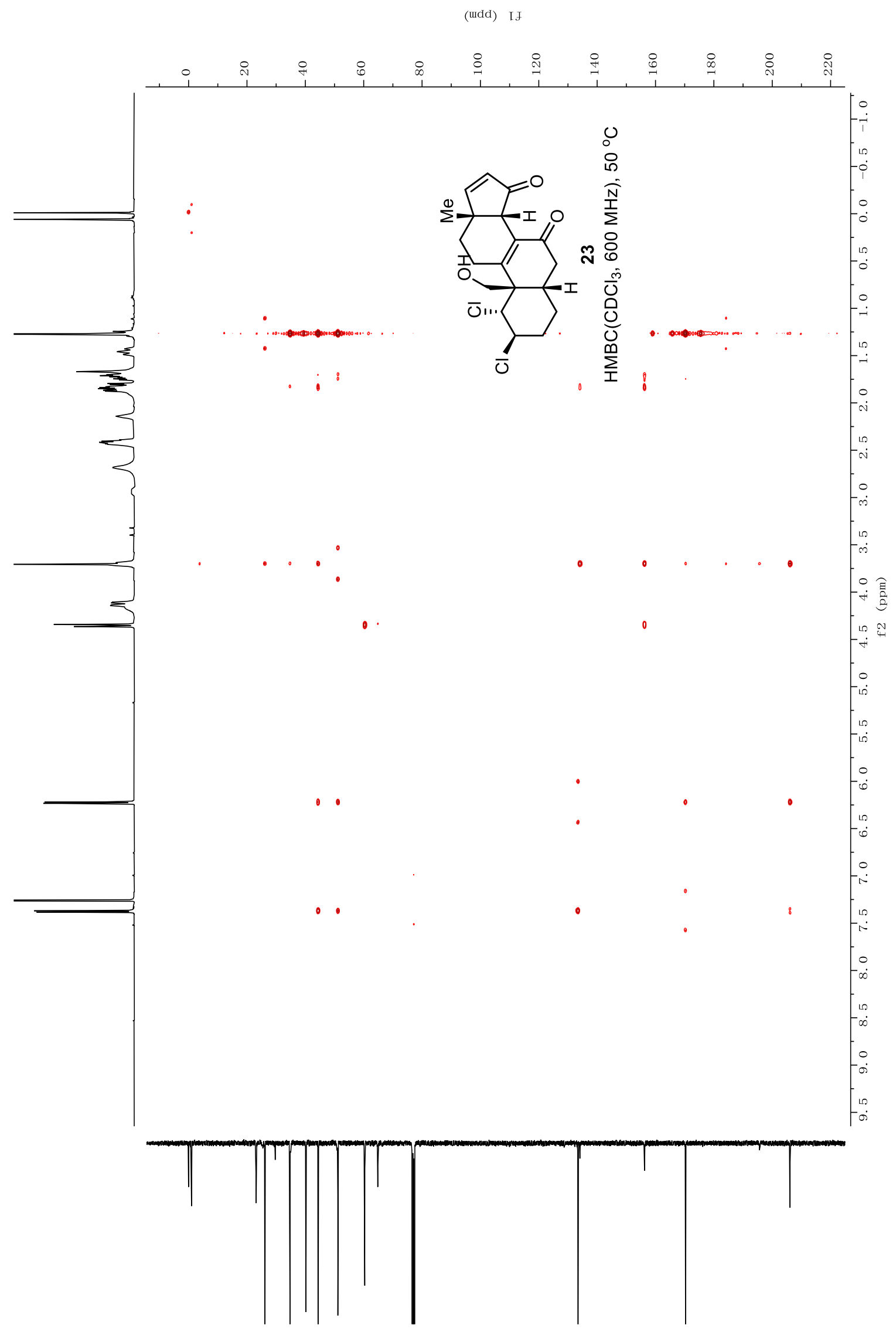




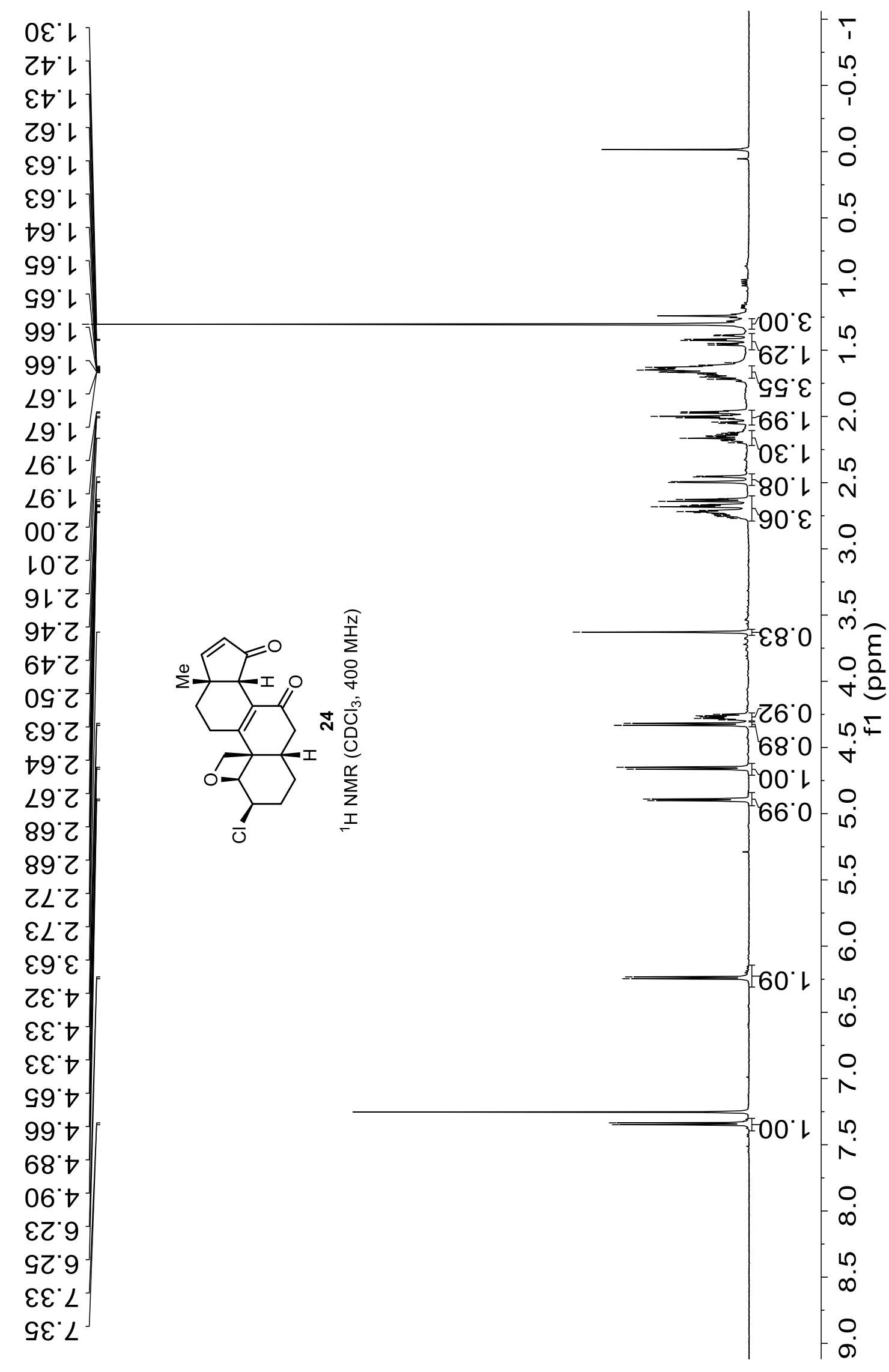



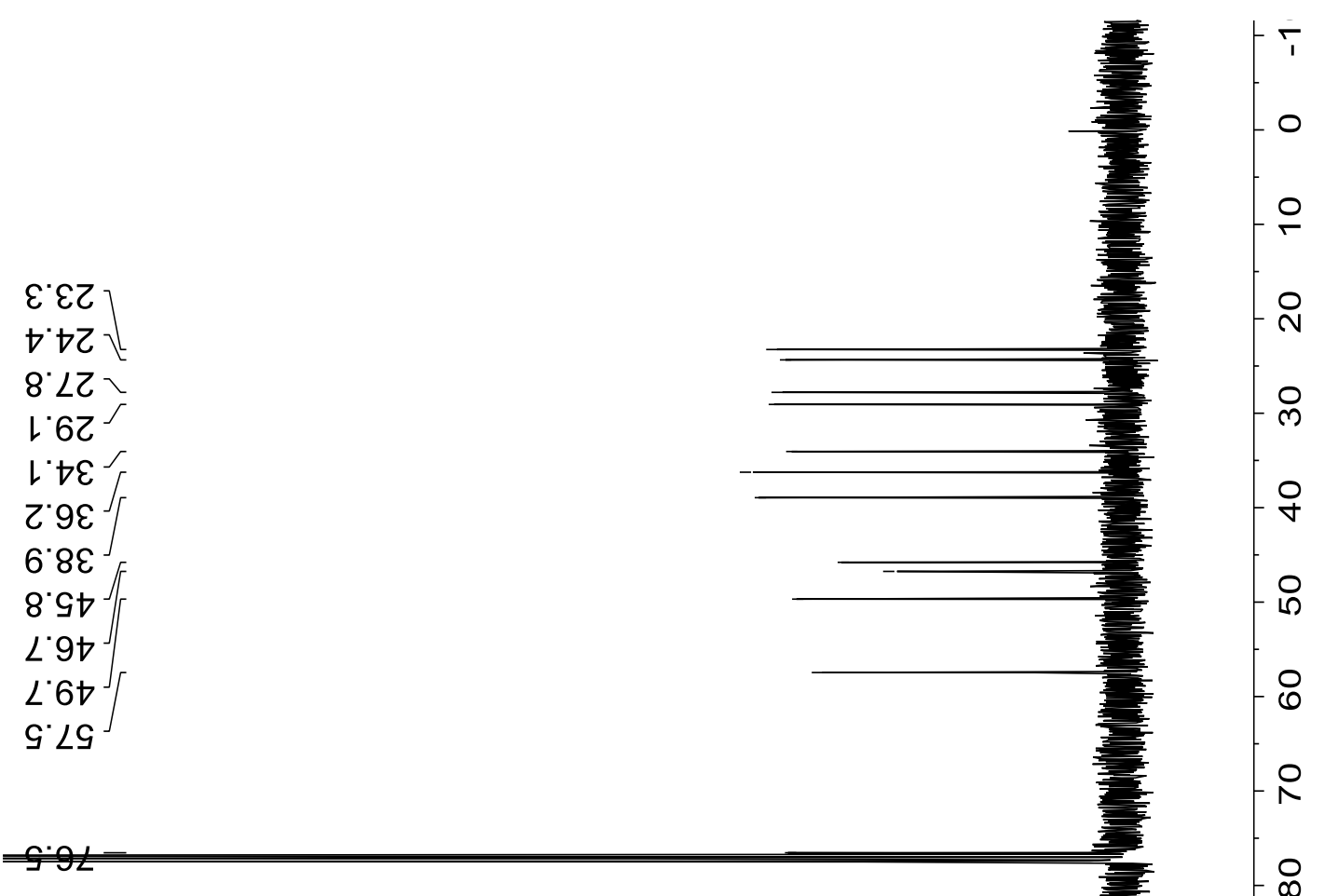

L'98-

G'Zยᄂ

$\nabla^{*} \nabla \varepsilon L^{-}$

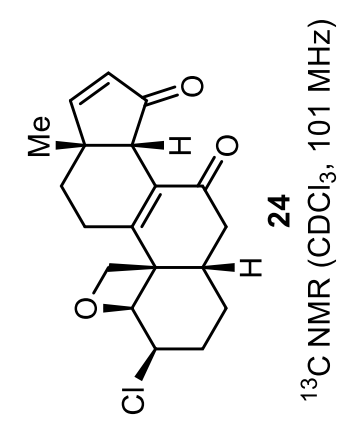

G'ZSL-

Z.0LL-

9 †6レ-

$8^{\circ} 902-$

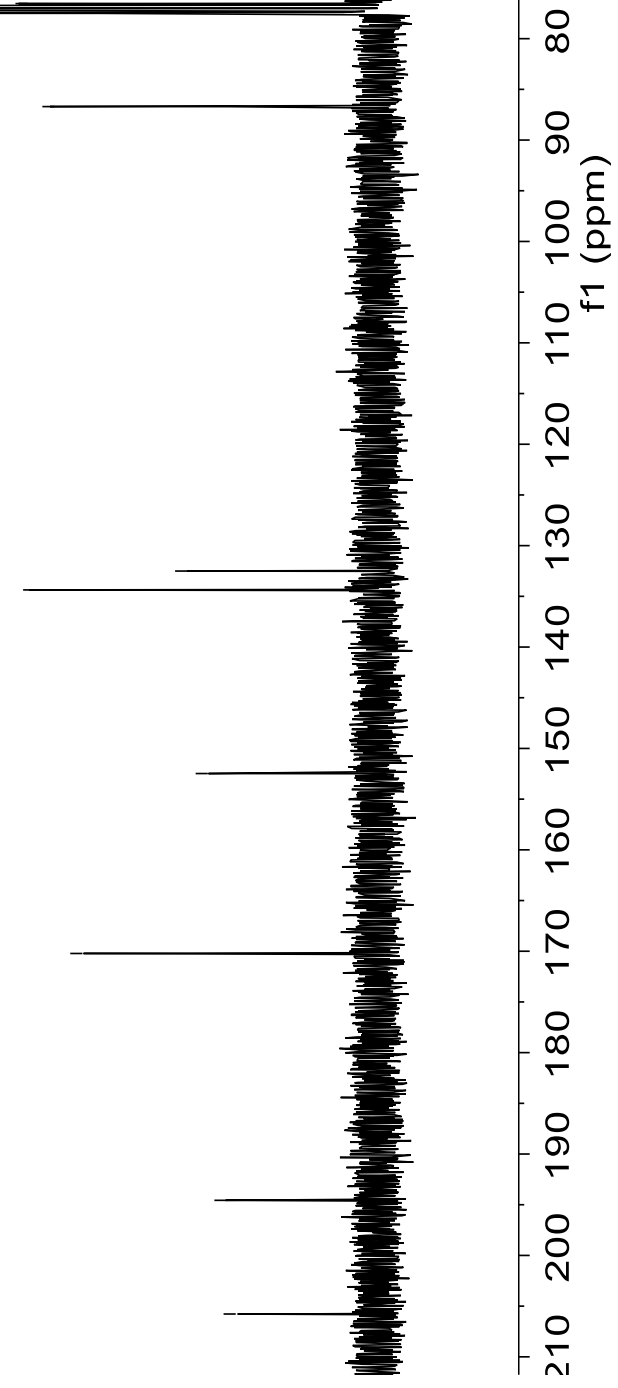




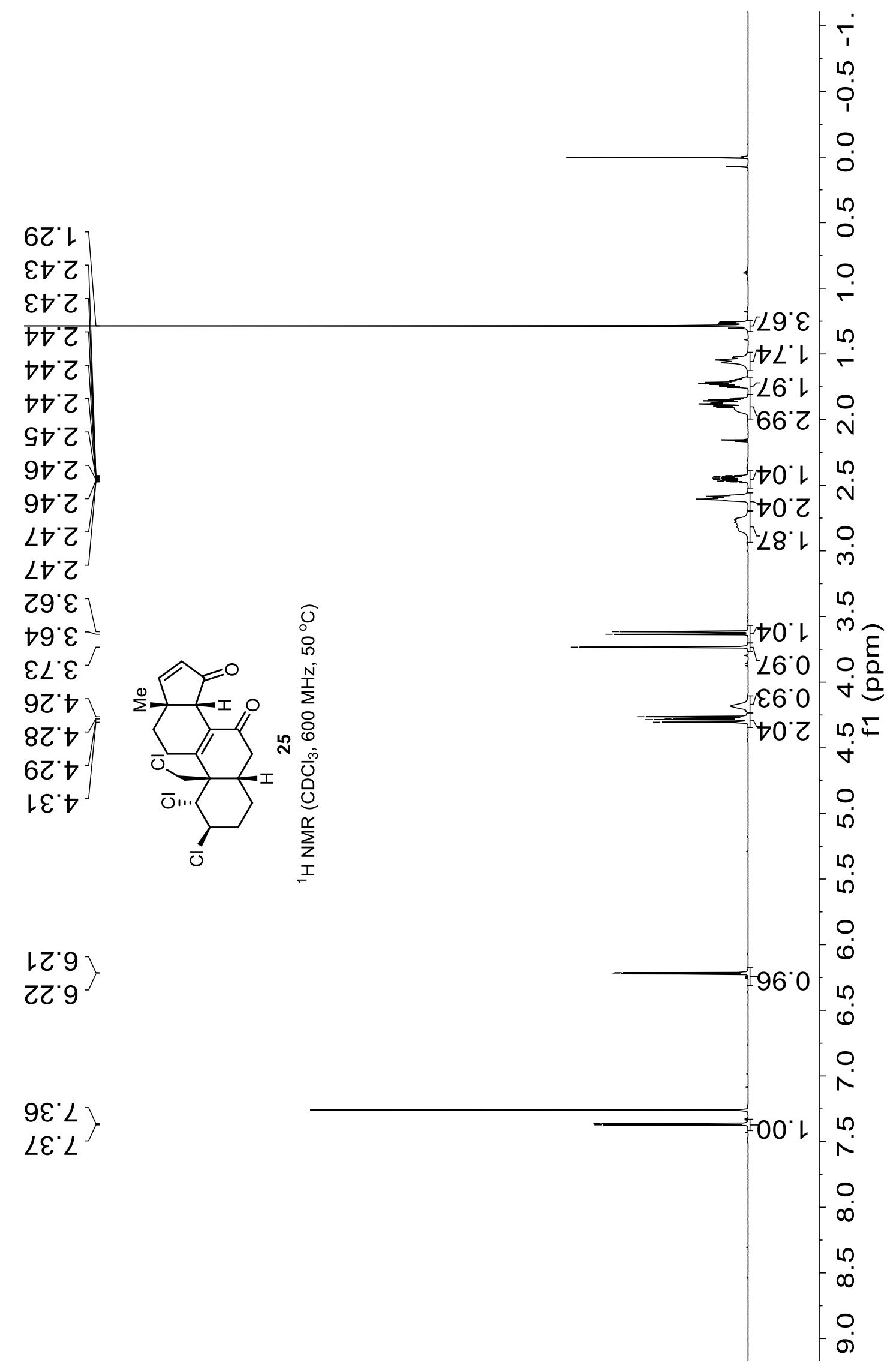




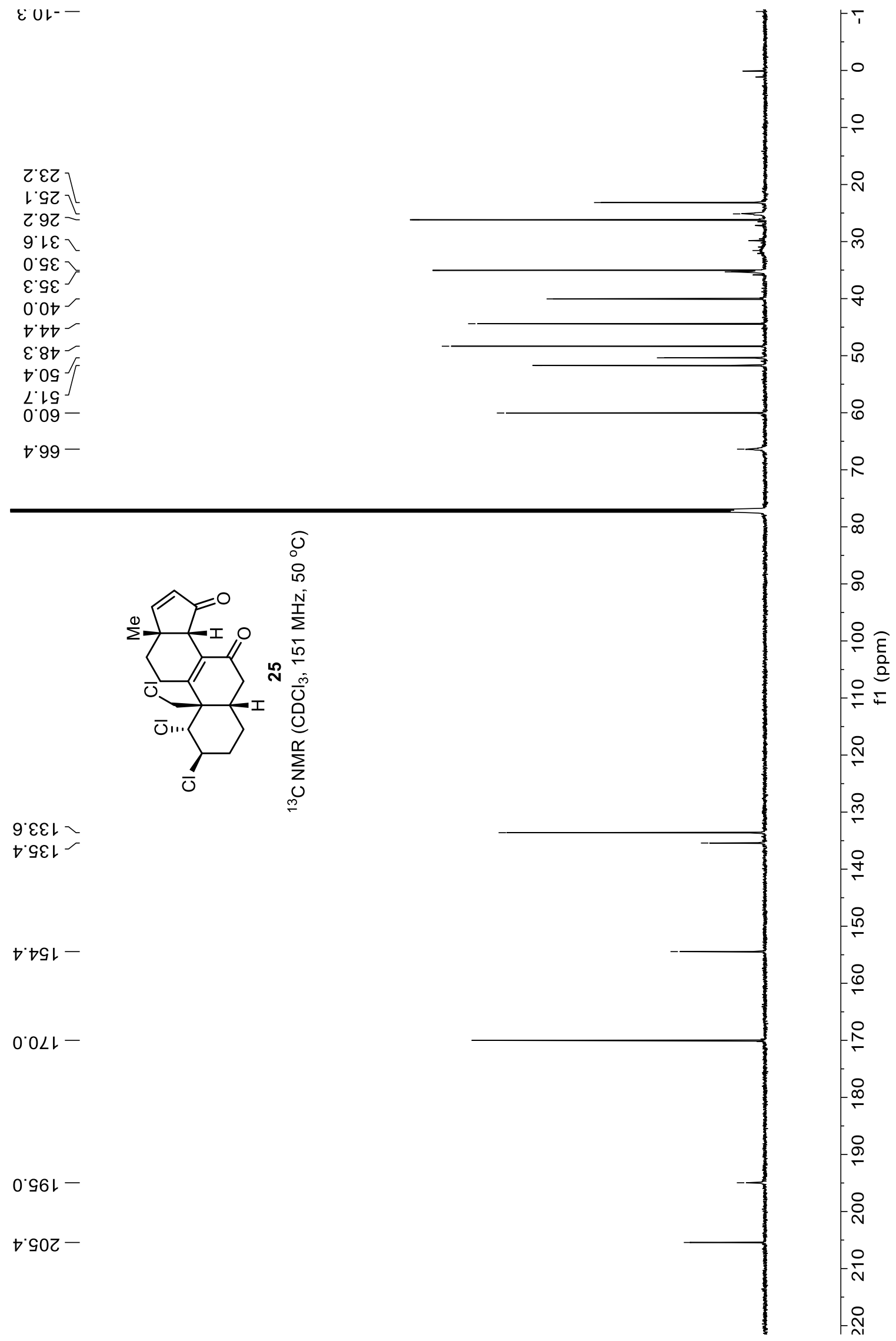




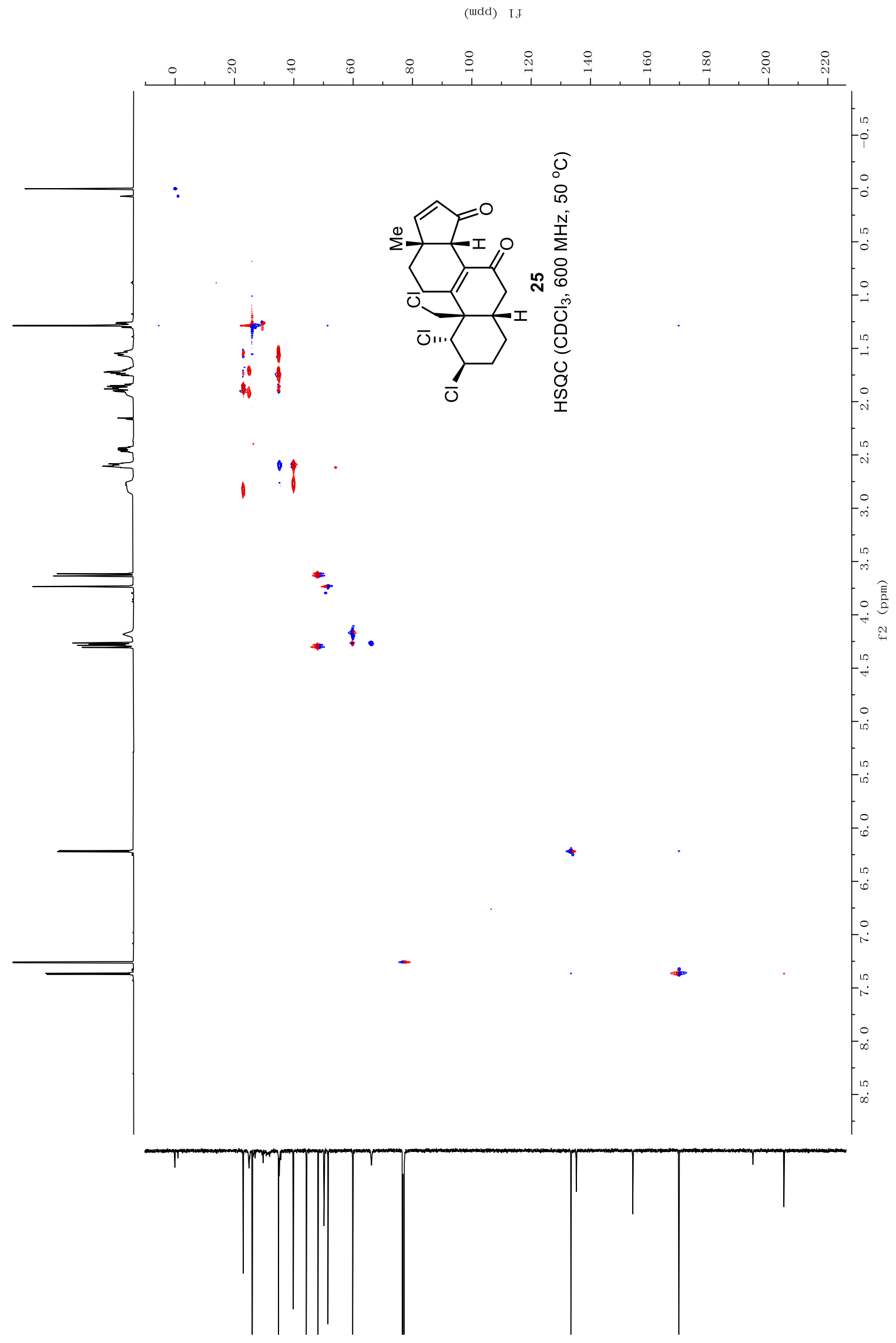




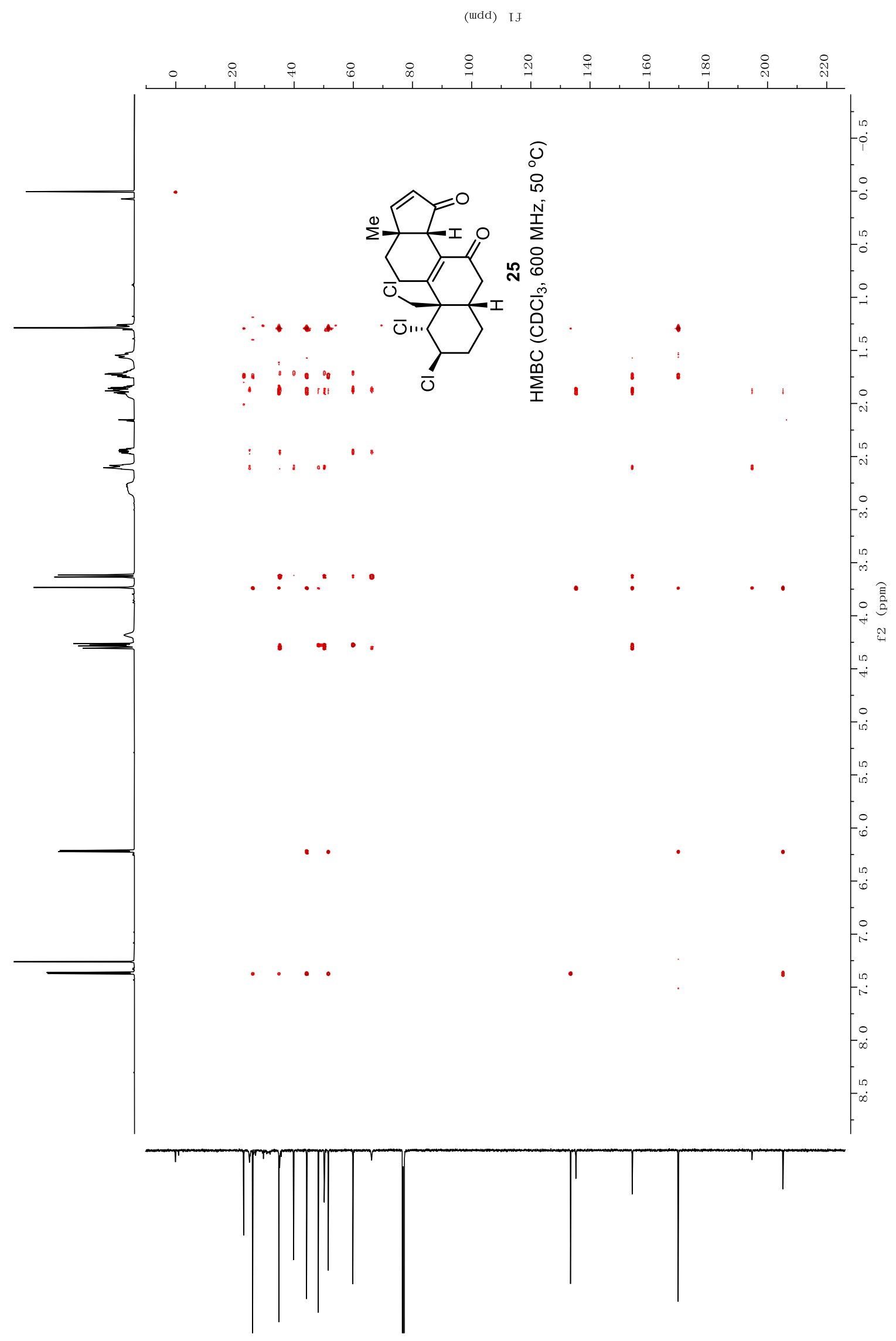




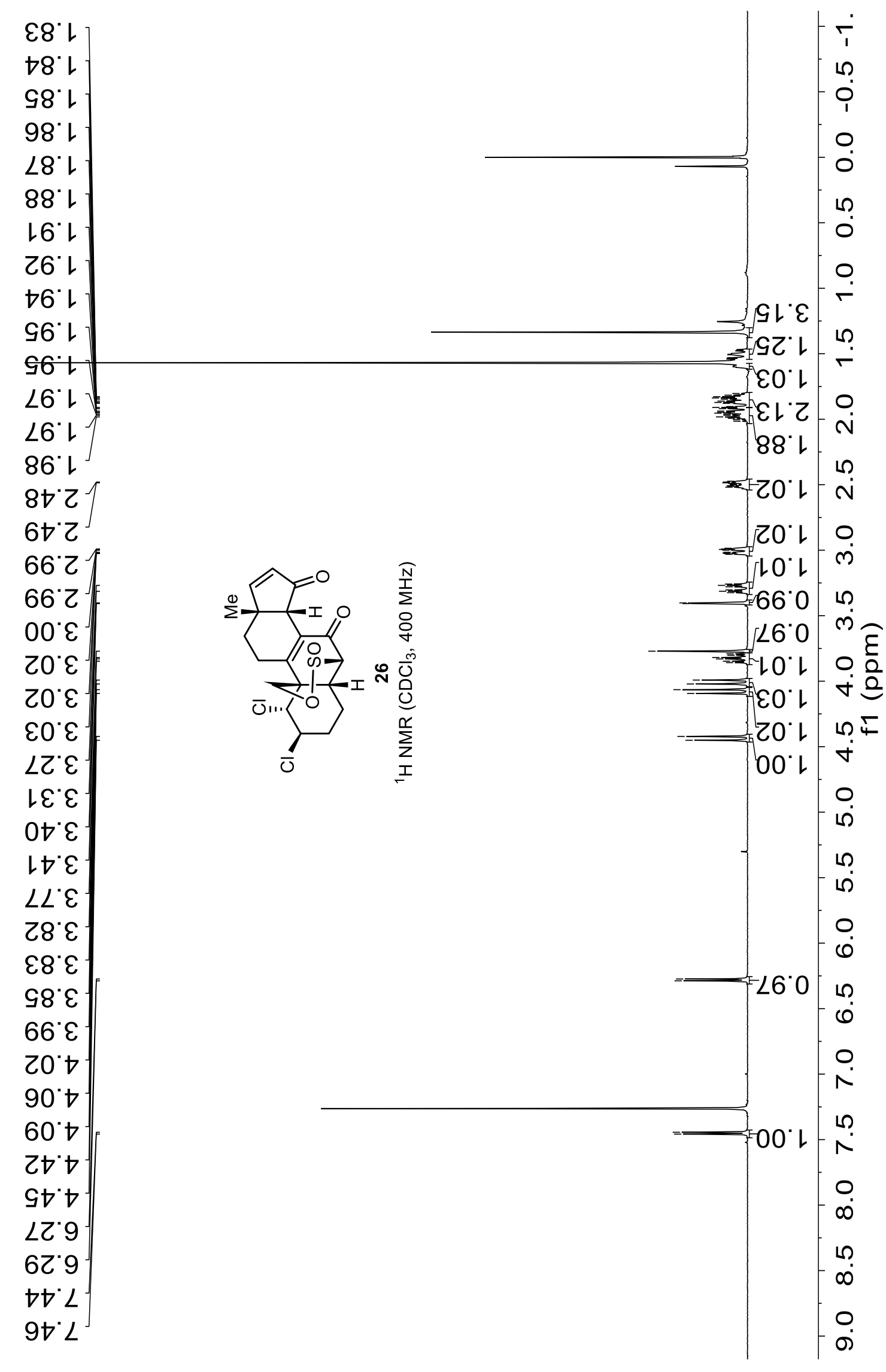



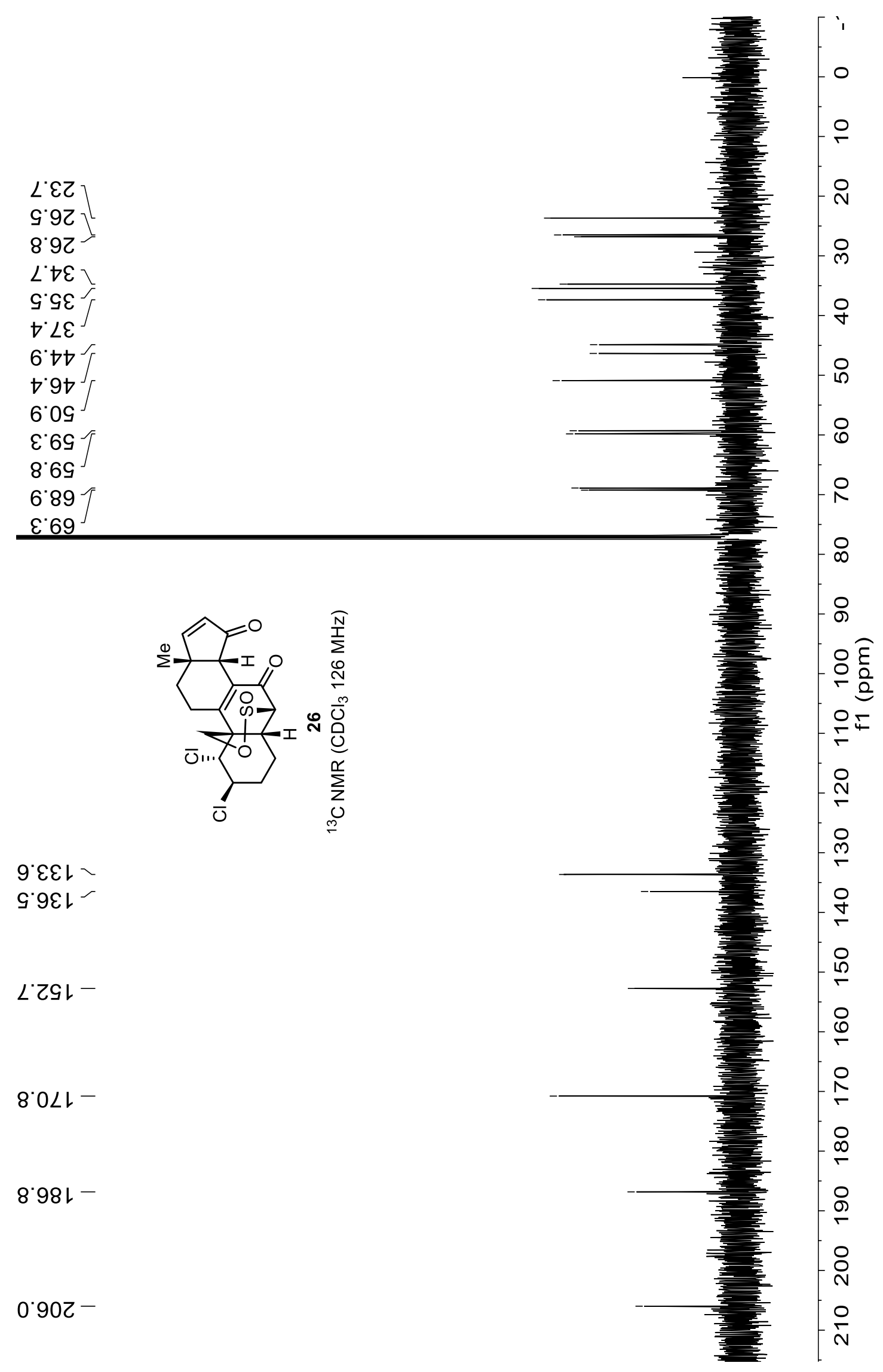


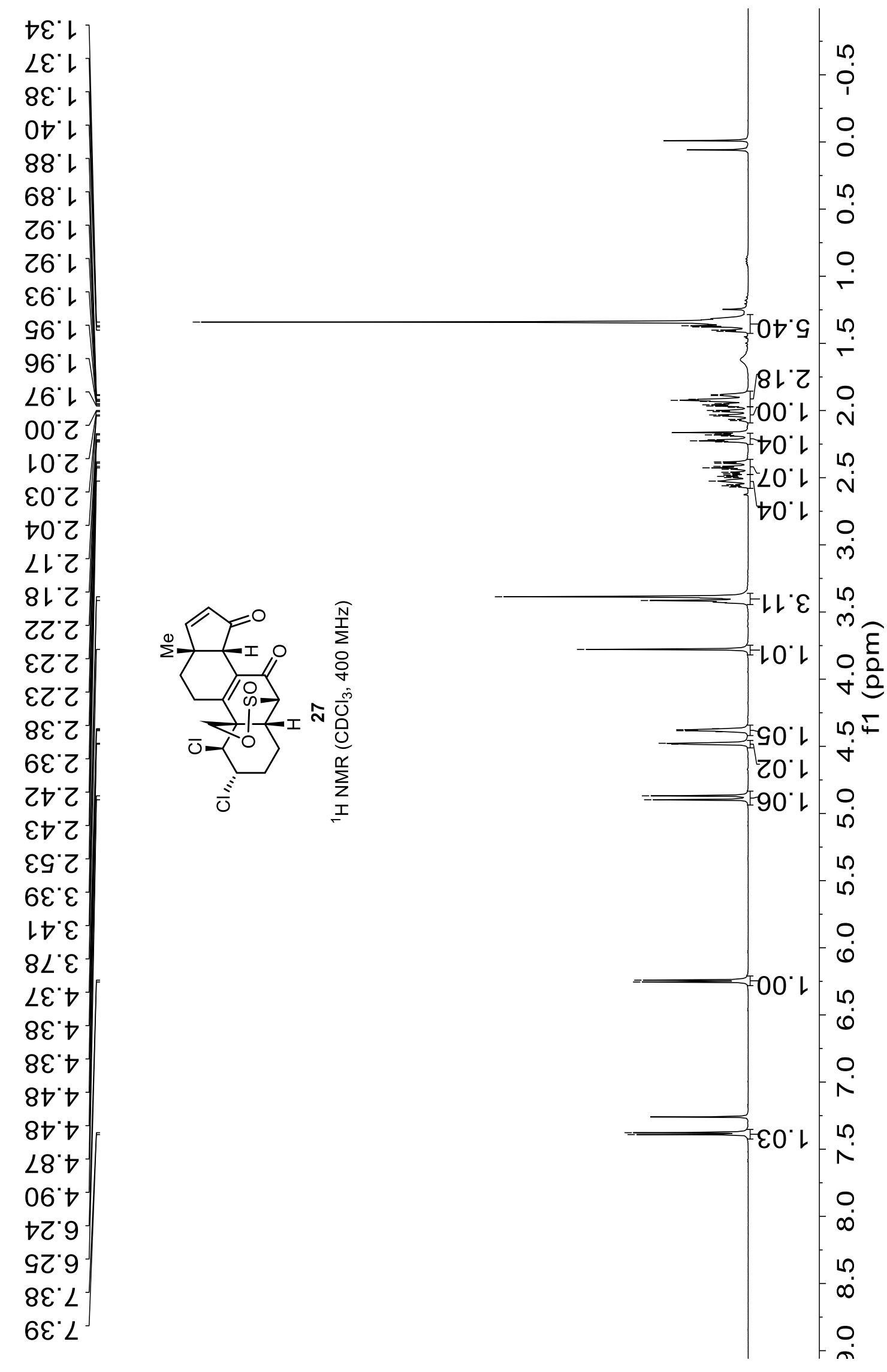




$$
\begin{aligned}
& \left.\begin{array}{l}
6.0 乙 \\
0.9 乙
\end{array}\right] \\
& \checkmark L Z \\
& 9^{\circ} \angle 乙 \frac{1}{5} \\
& 9.82 \\
& \nabla \\
& 8^{\circ} \varepsilon t \backslash \\
& \tau \cdot 9 t- \\
& 86 b^{-} \\
& \text {L89 } \\
& 6.69 T \\
& \text { 6.69 } \\
& \text { OLL- }
\end{aligned}
$$

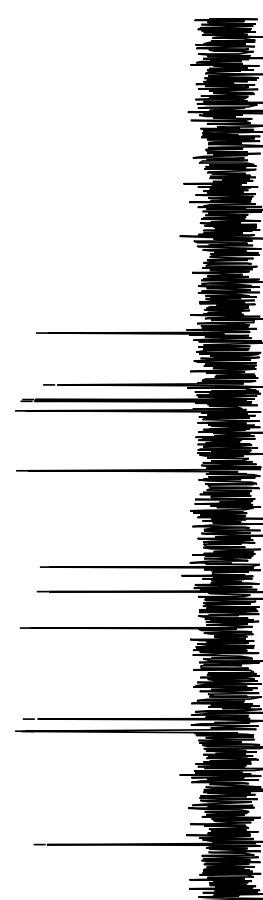

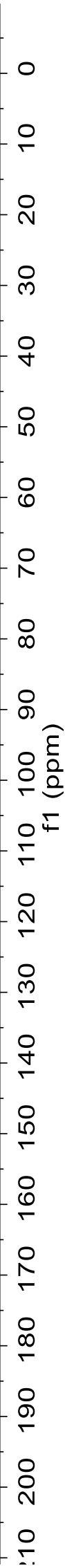

$\left.\begin{array}{l}9^{\circ} \triangleright \varepsilon\llcorner \\ 8^{\circ} \triangleright \varepsilon\llcorner\end{array}\right\}$

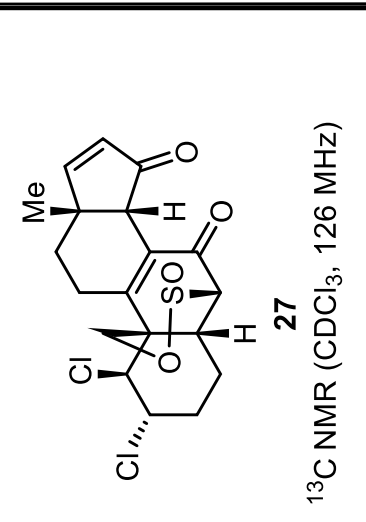

0.9Gレ-

900L-

$\varepsilon^{\prime} \angle 8 L-$

$8^{\circ} \triangleright 0 Z-$

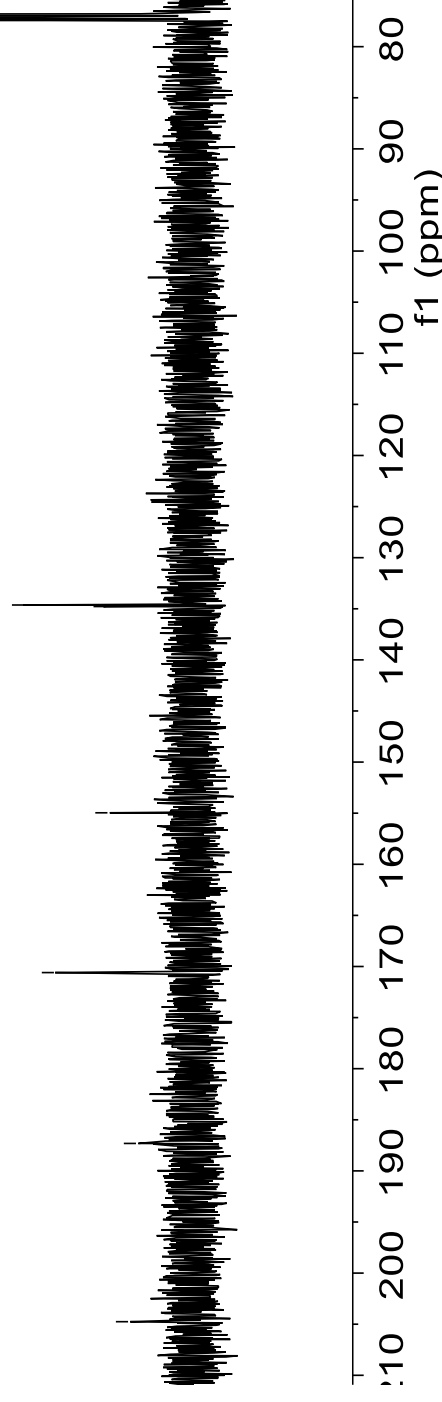




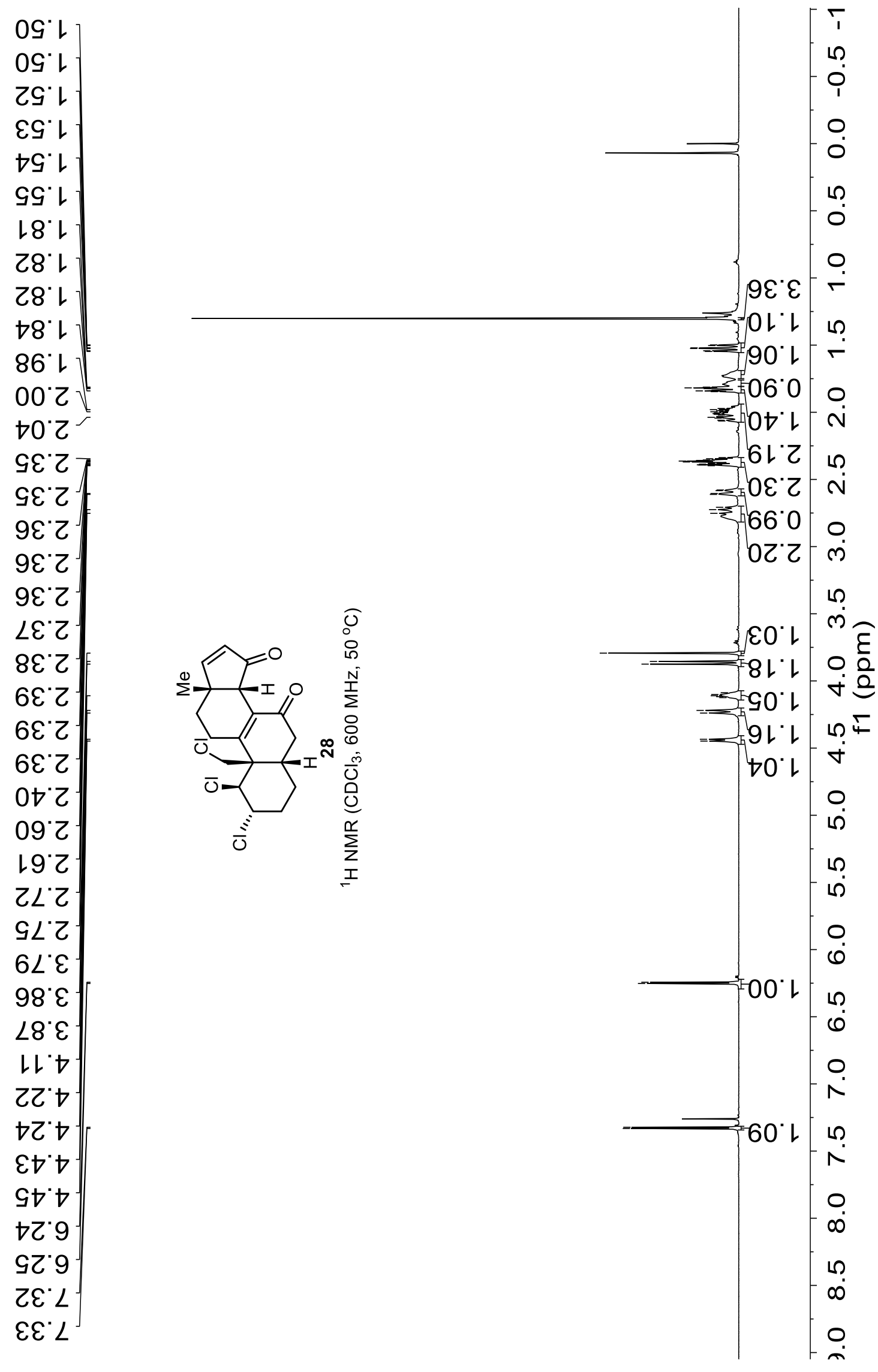




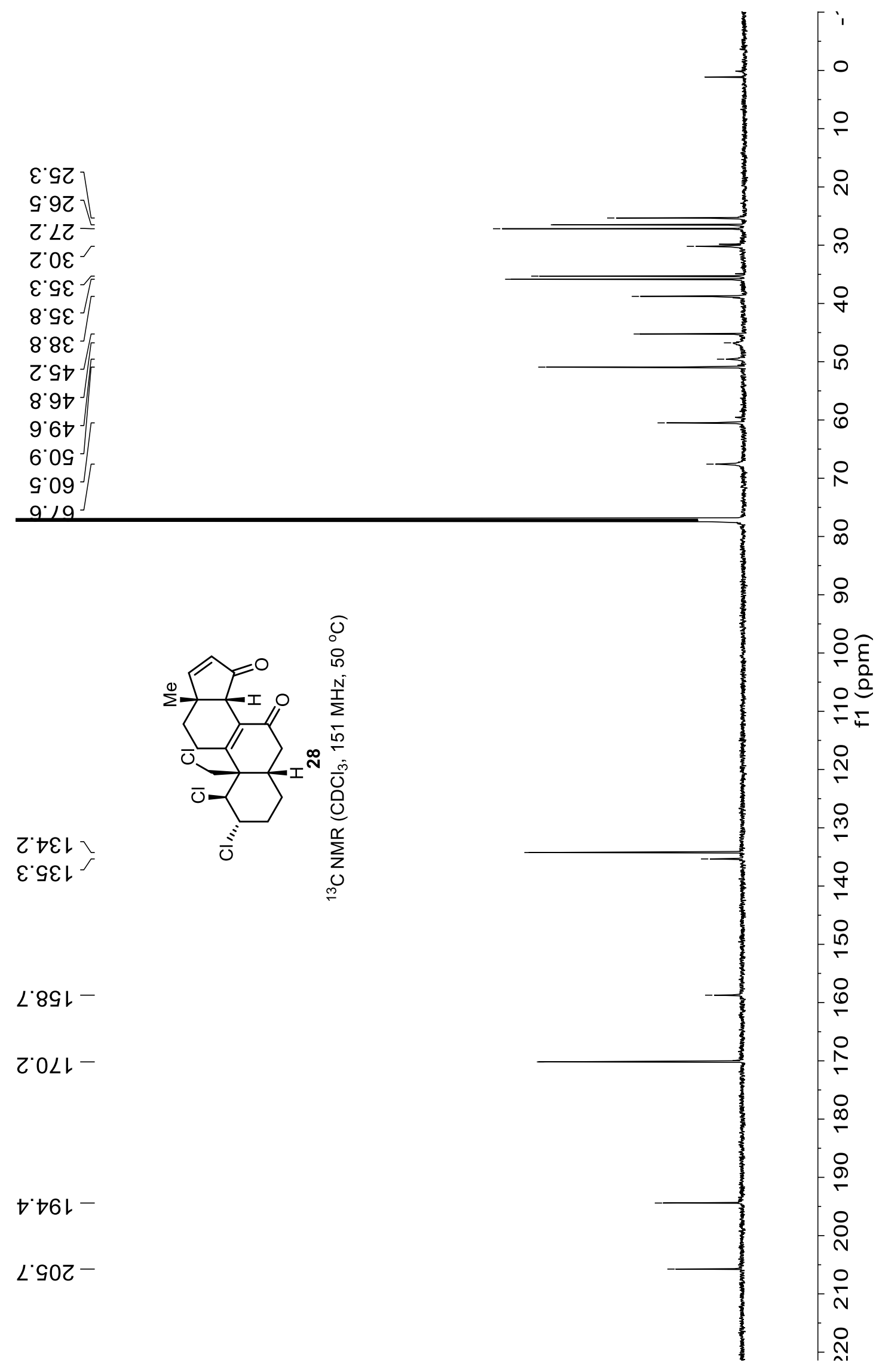




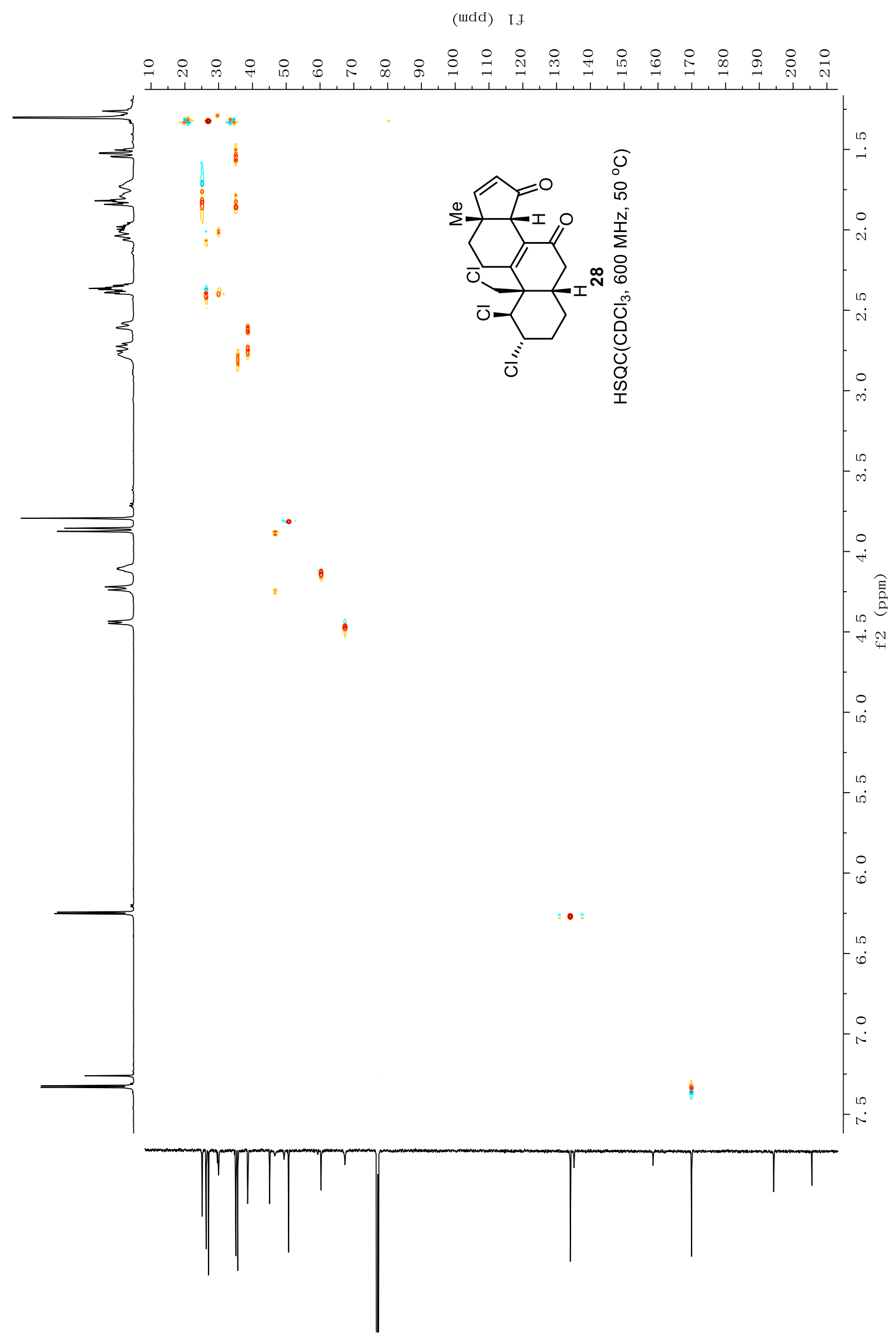




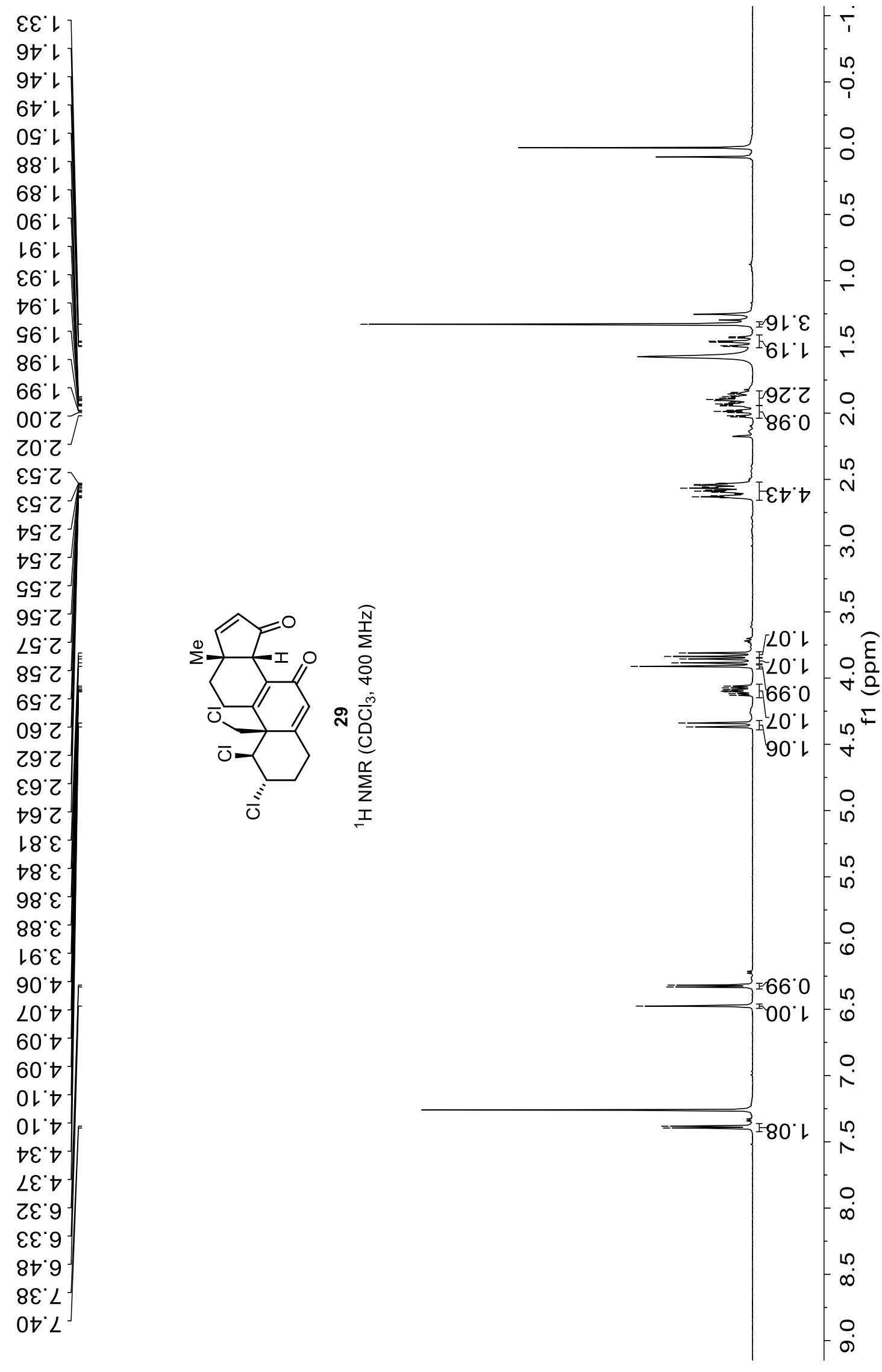



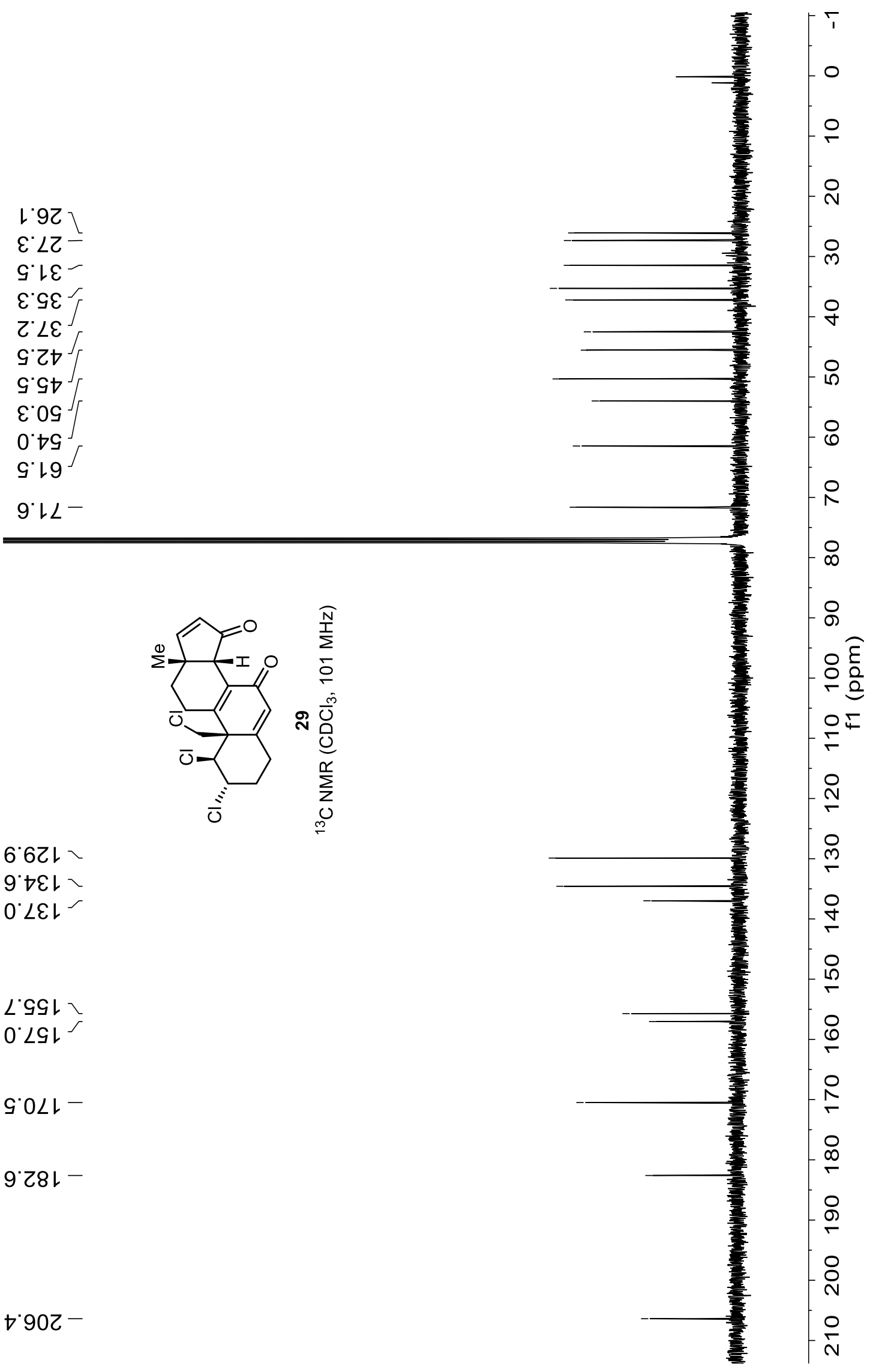


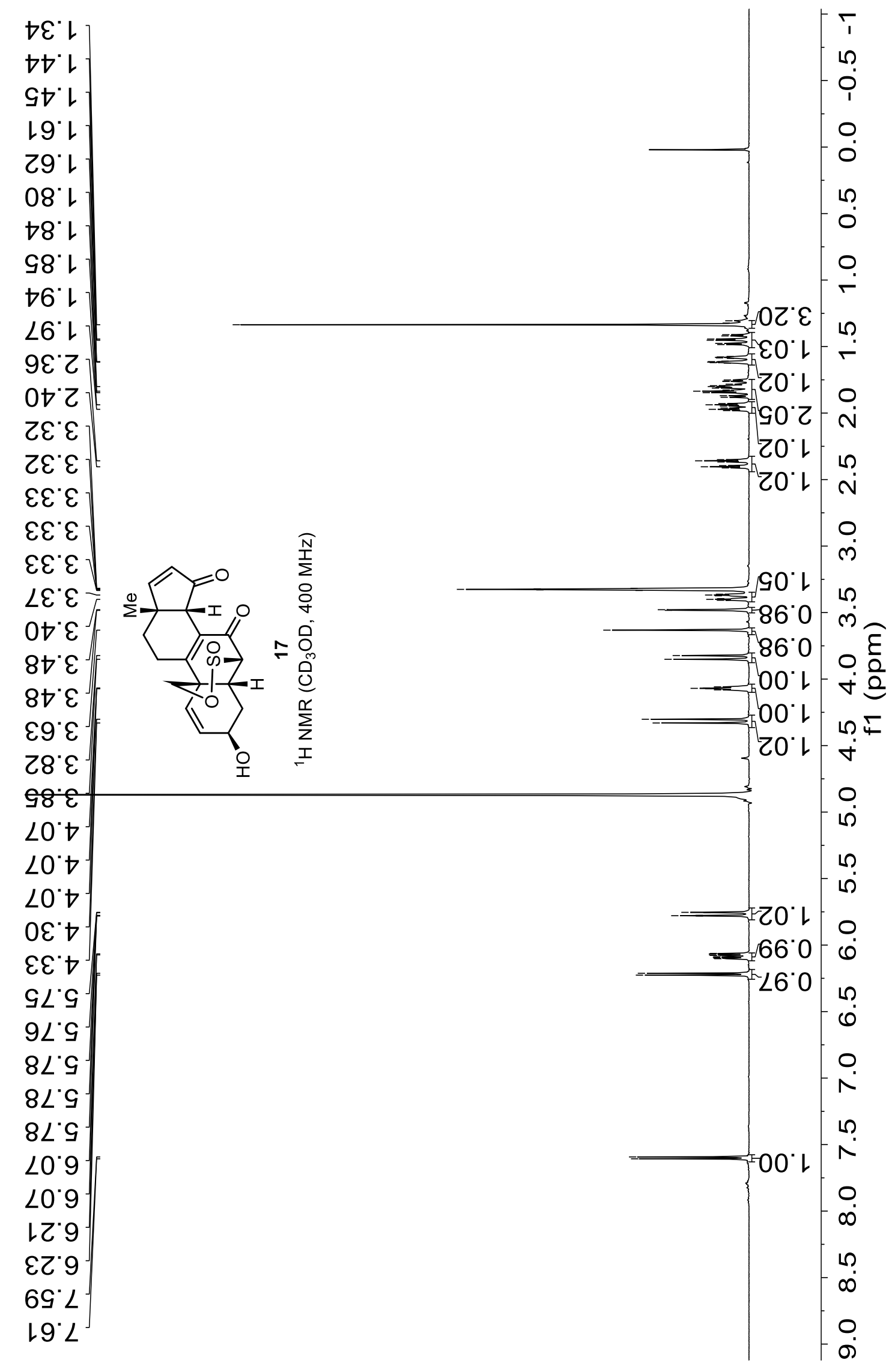



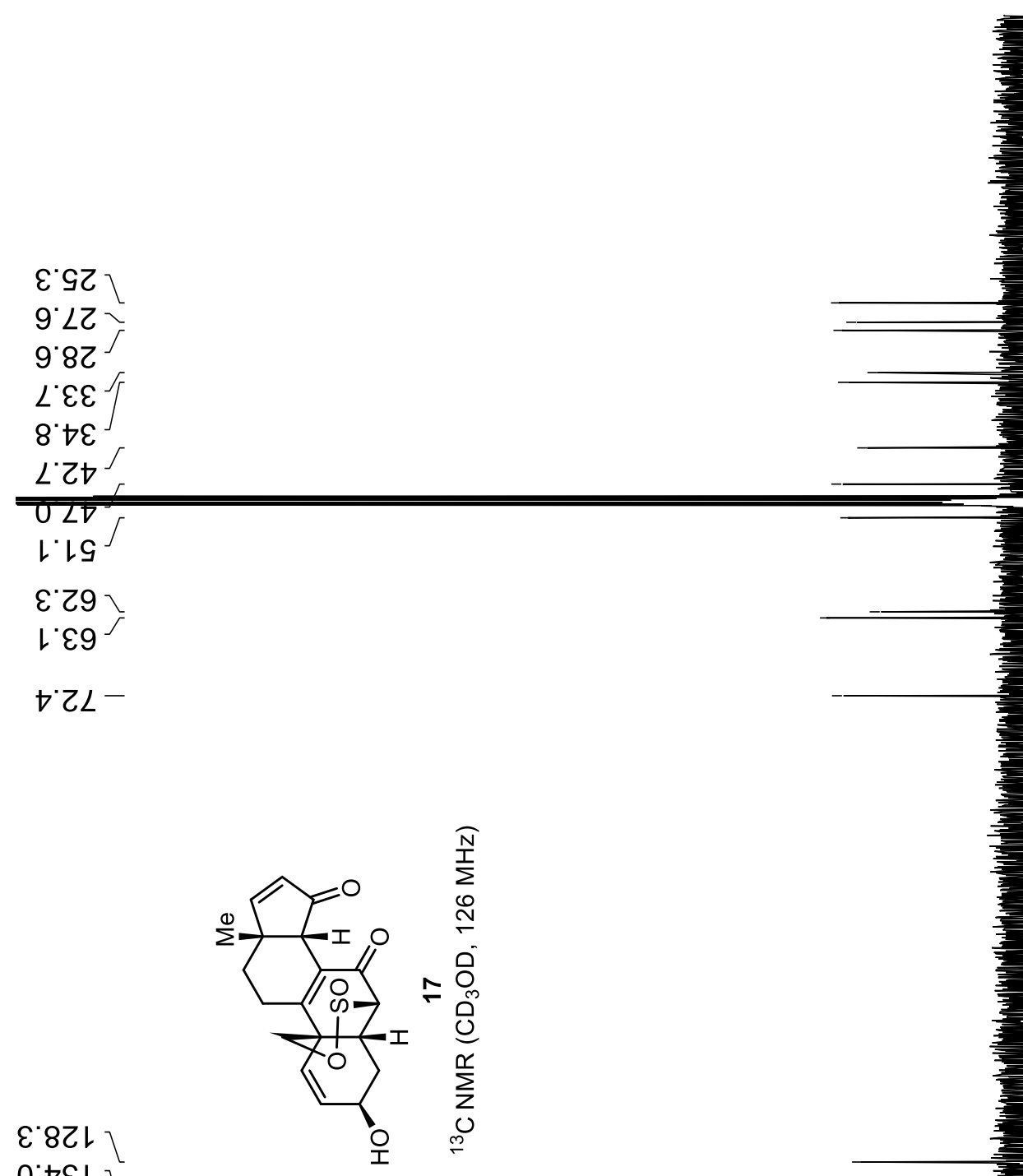

$\varepsilon \cdot 8 Z L$

$0 \circ \varepsilon L$

レ๋ยレ

$0 \subseteq \mathcal{G}$

Z'89レ-

$8^{\circ} \varepsilon \angle L-$

乙68 -

$\mathrm{S}^{\prime} 802^{-}$

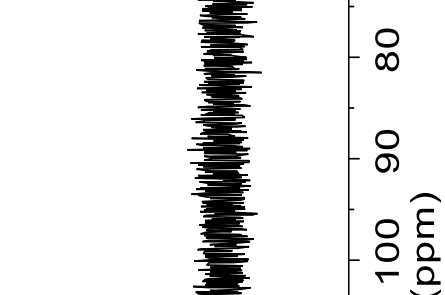

을

은ㄷㄴ

운

$\stackrel{-}{\sim}$

을
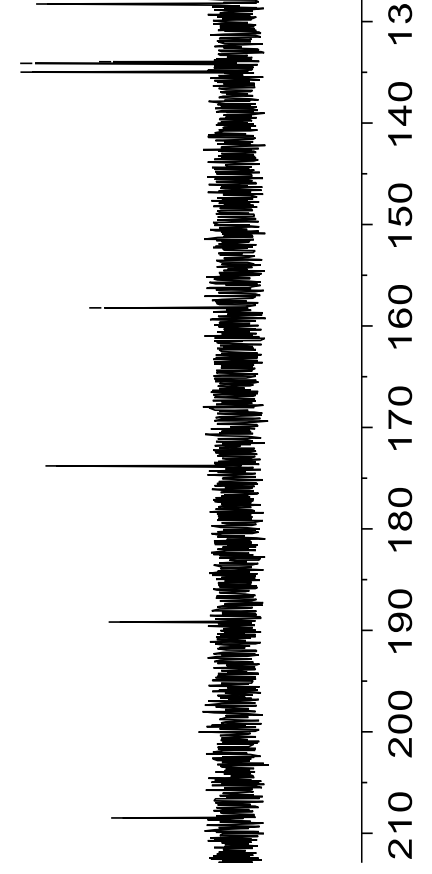


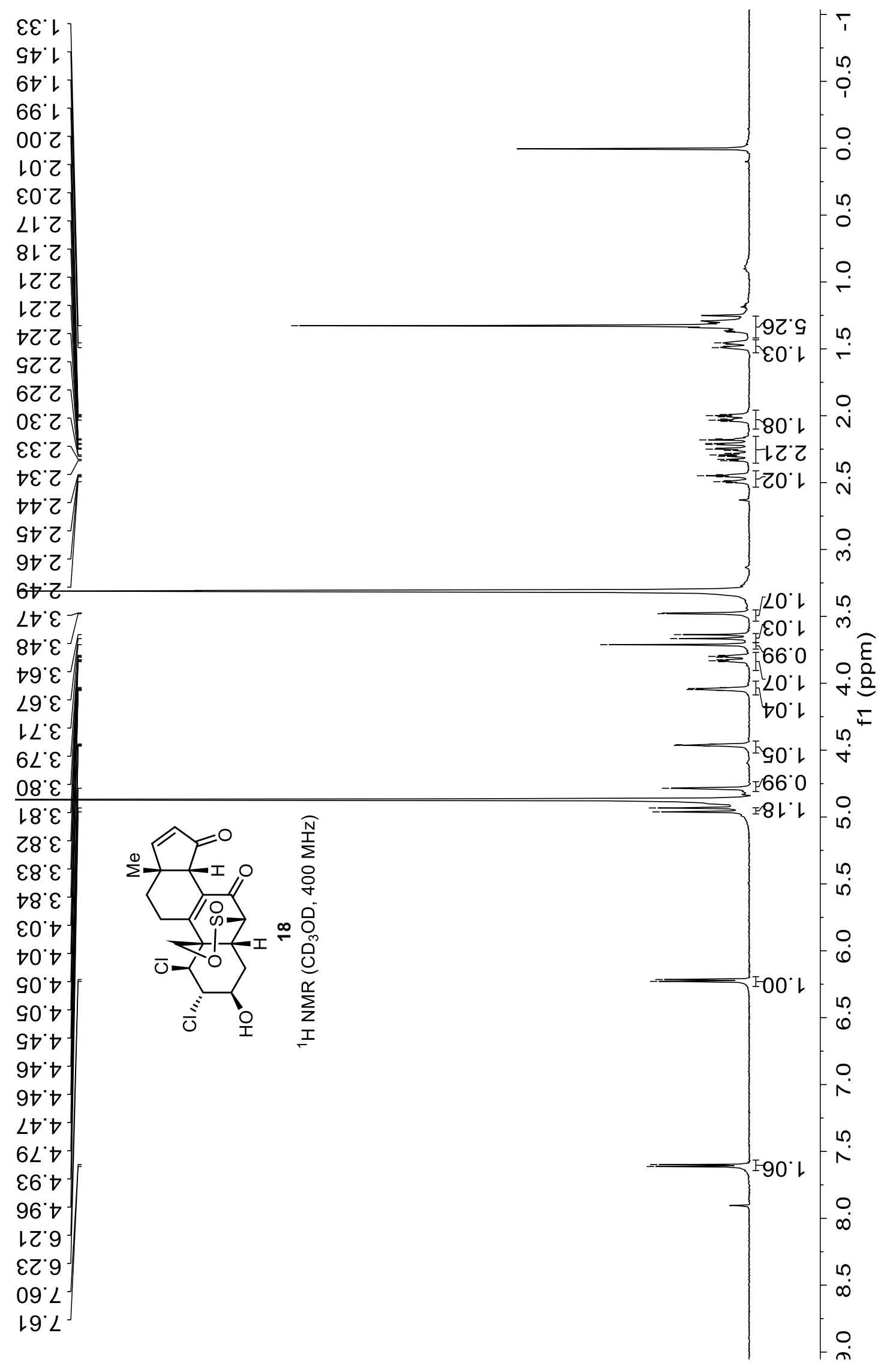




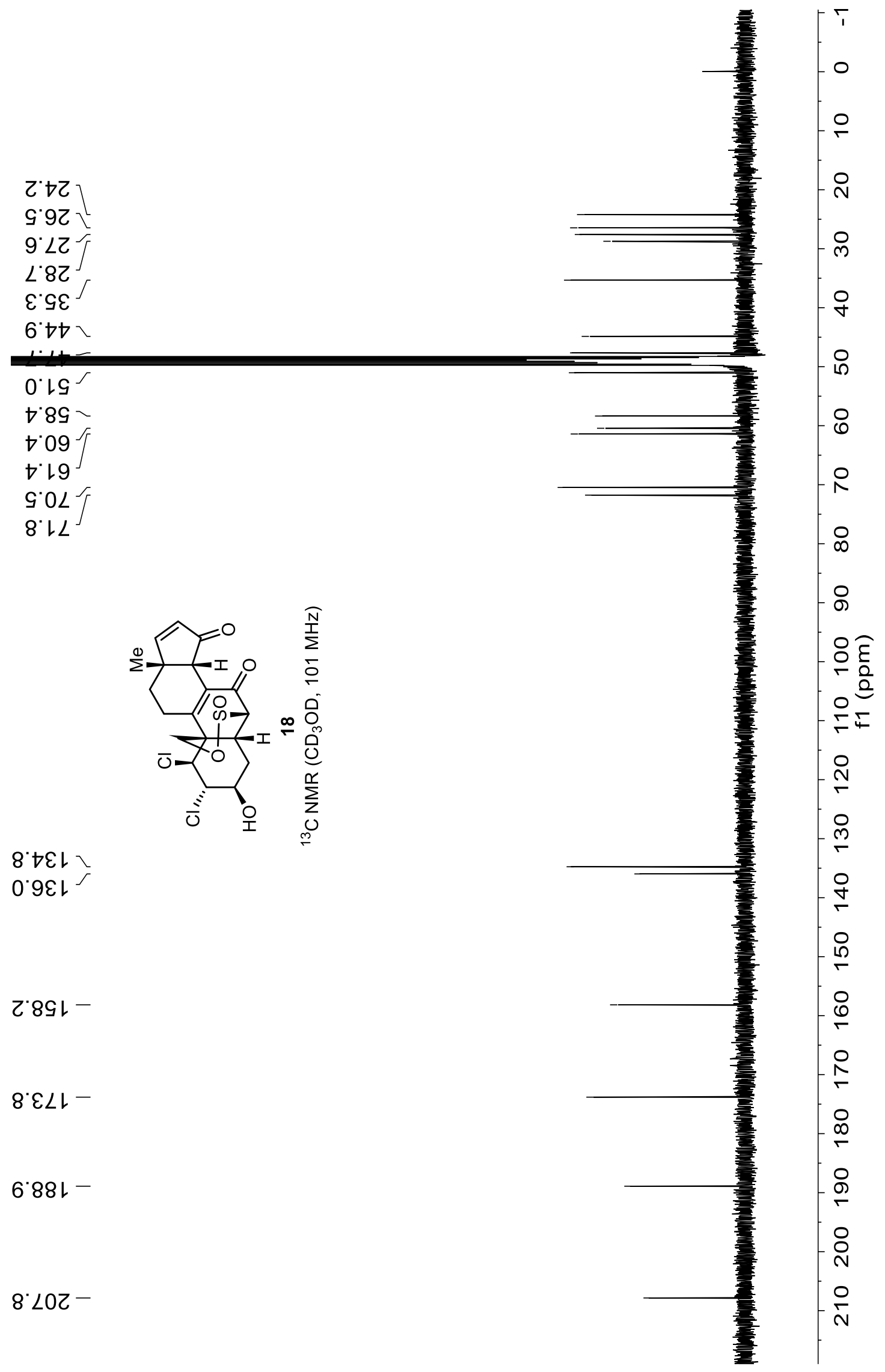




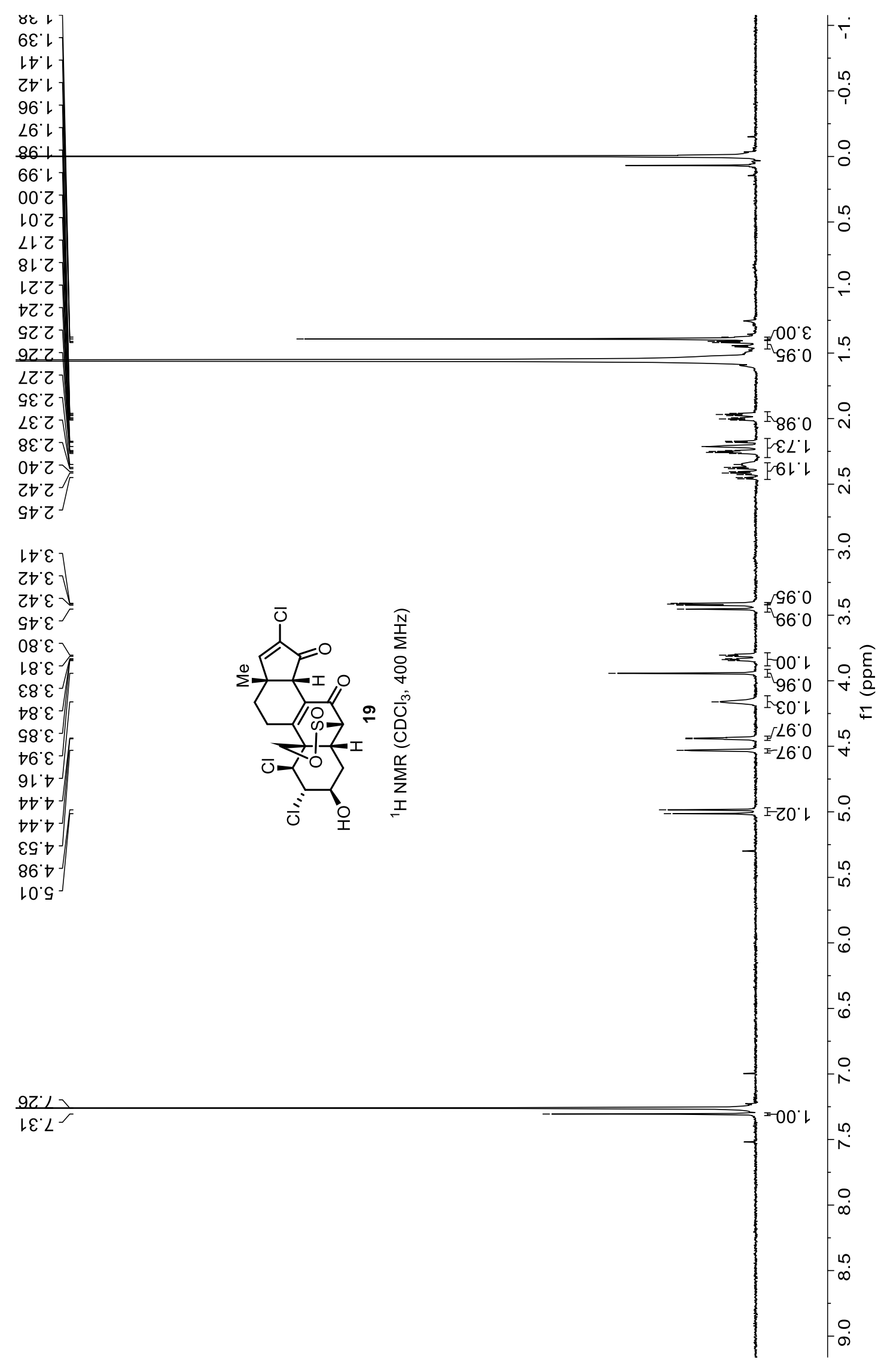




$$
\begin{aligned}
& \begin{array}{l}
乙^{*} \varepsilon 乙 \\
8^{\cdot} 9 乙
\end{array} \\
& 9 . \angle Z] \\
& 0.82
\end{aligned}
$$

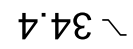

$$
\begin{aligned}
& \left.\begin{array}{l}
8^{\circ} \varepsilon \downarrow \\
\sigma^{\circ} \varepsilon t
\end{array}\right] \\
& \text { l.6t } \\
& \begin{array}{l}
9 . \angle G \\
969
\end{array} \\
& \angle 69 \\
& \begin{array}{l}
0.02 \\
9.02
\end{array}
\end{aligned}
$$
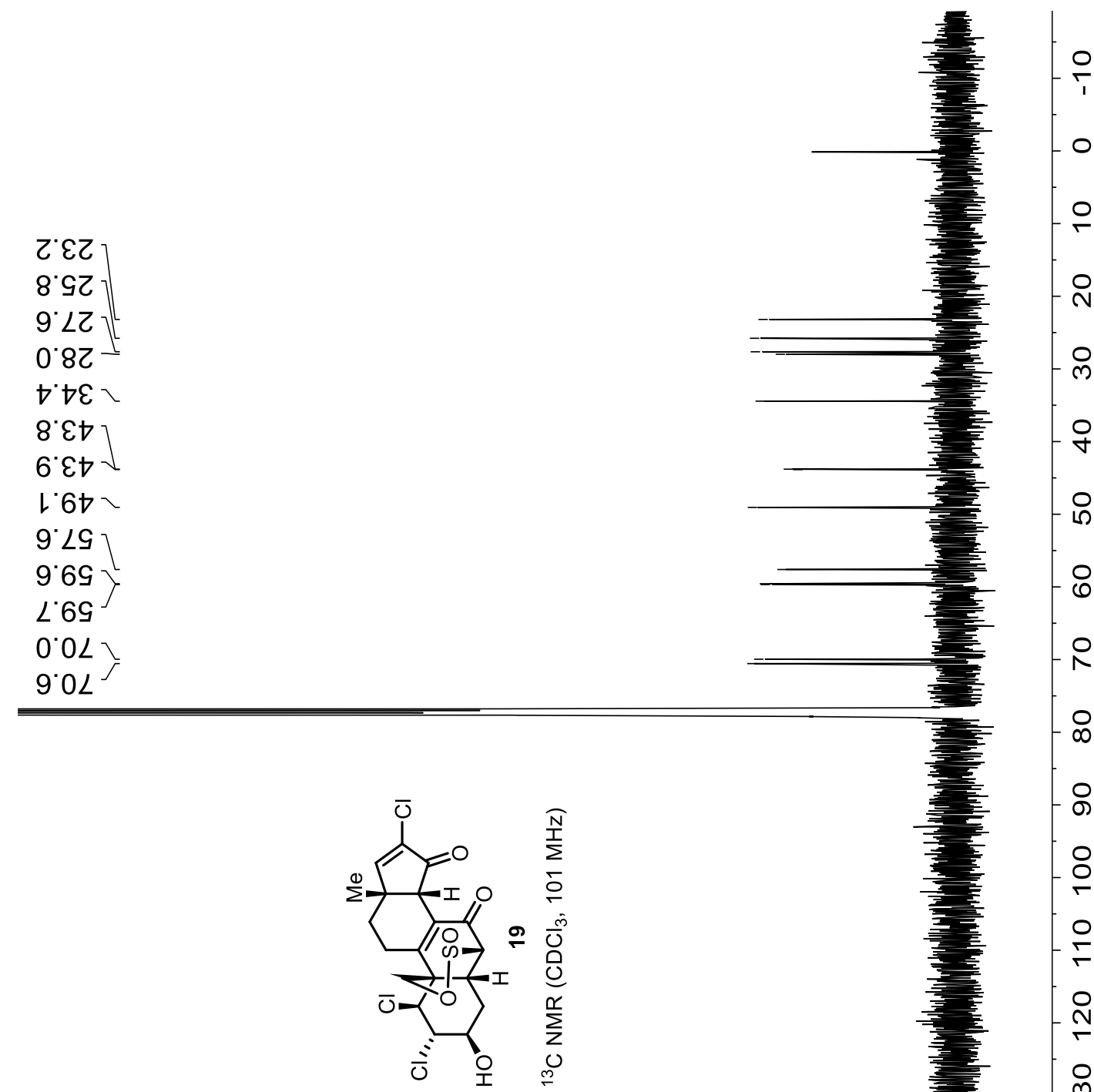

9 ๑レレ

乙.9ع

0.GSเ-

0.89 -

L'98L-

†・96レー
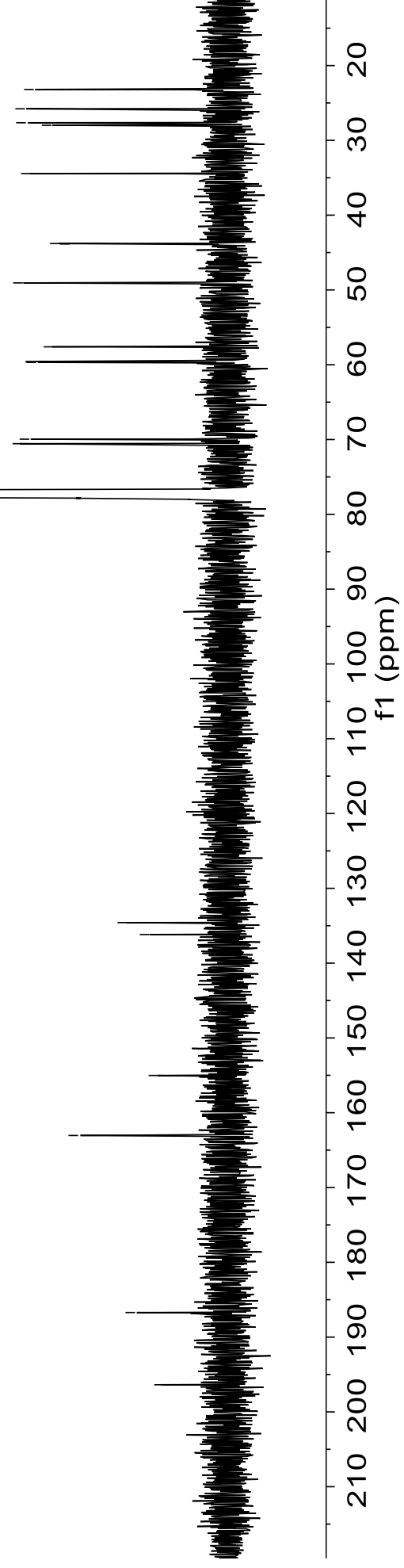


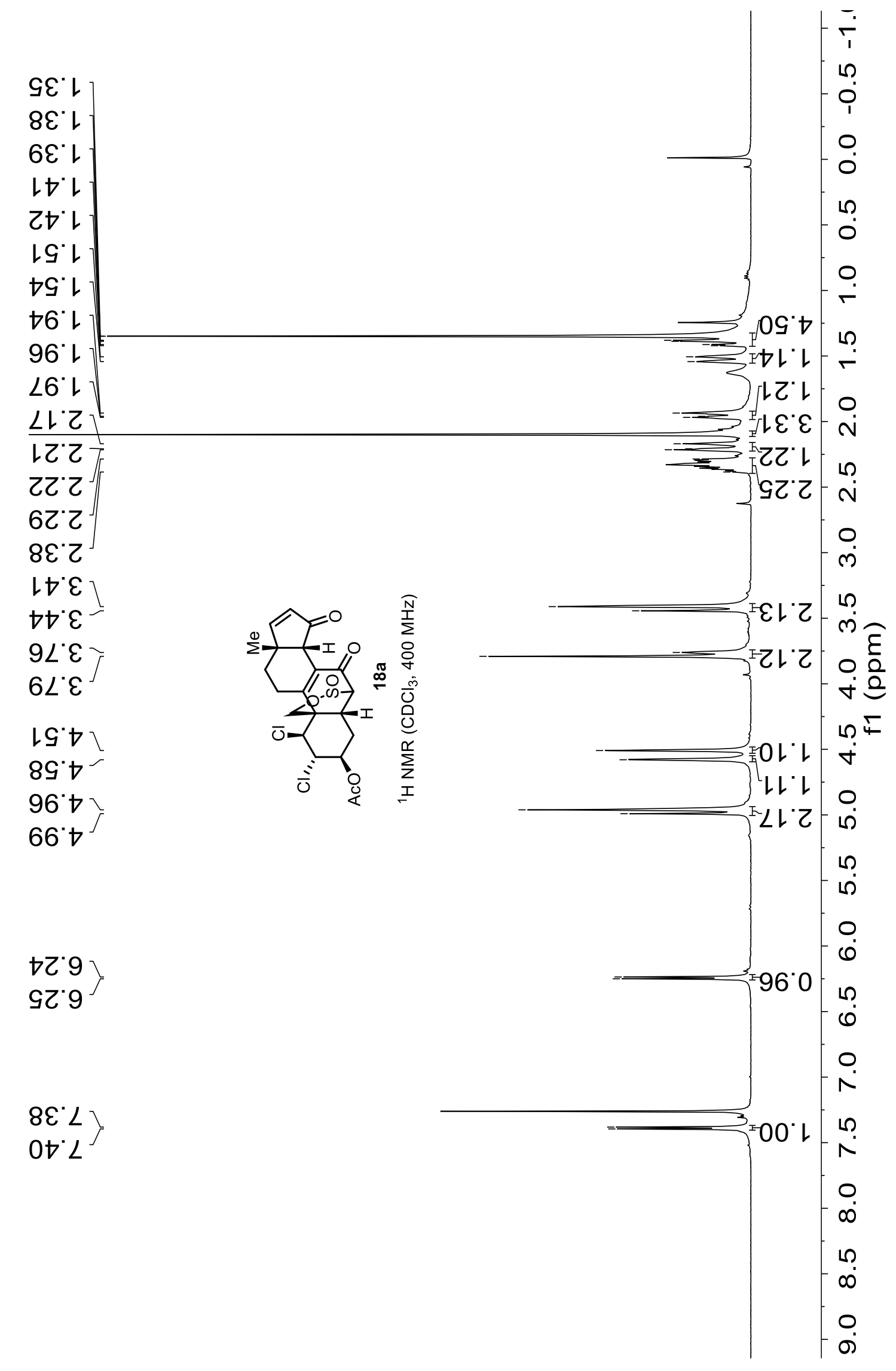




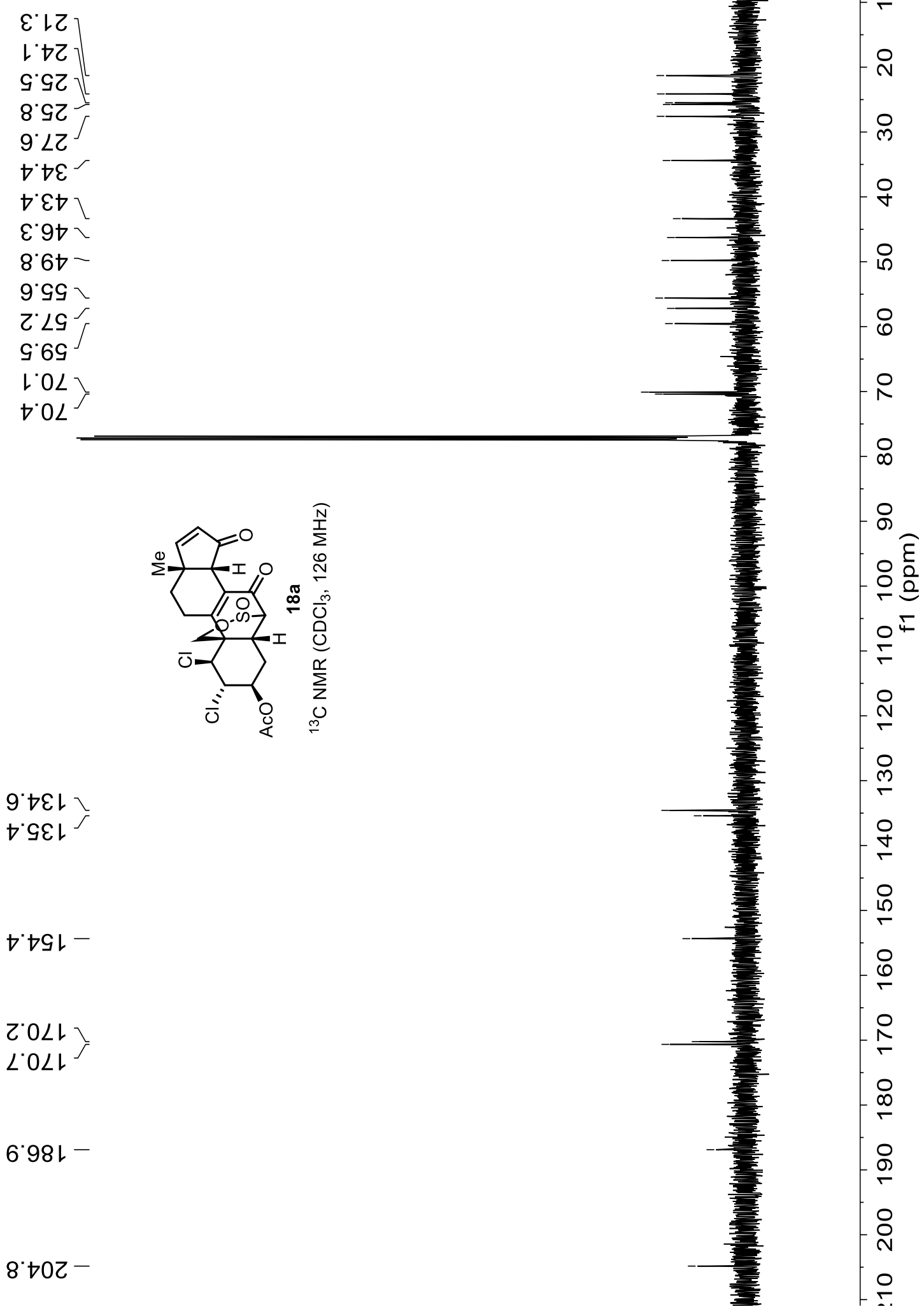




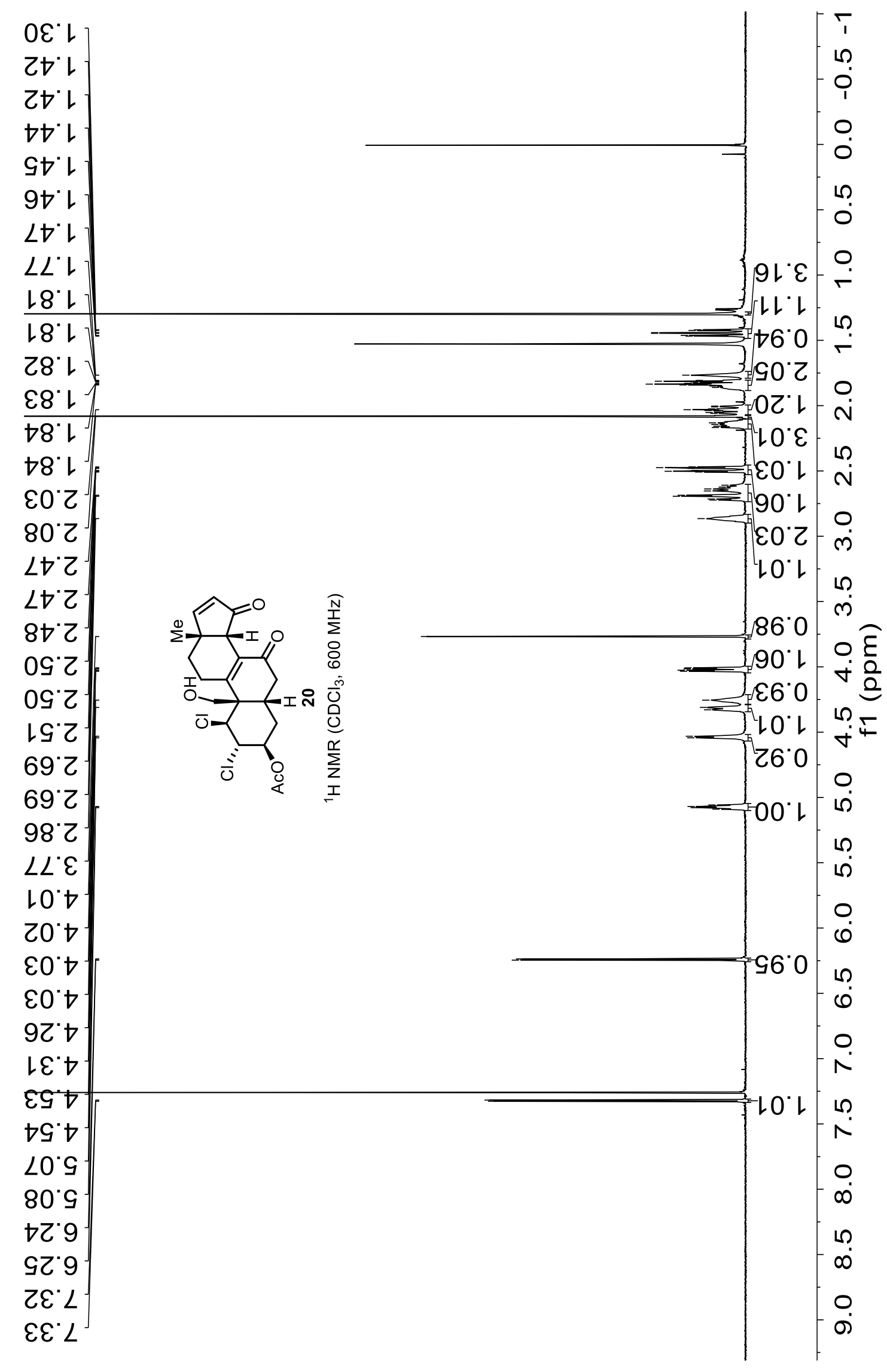




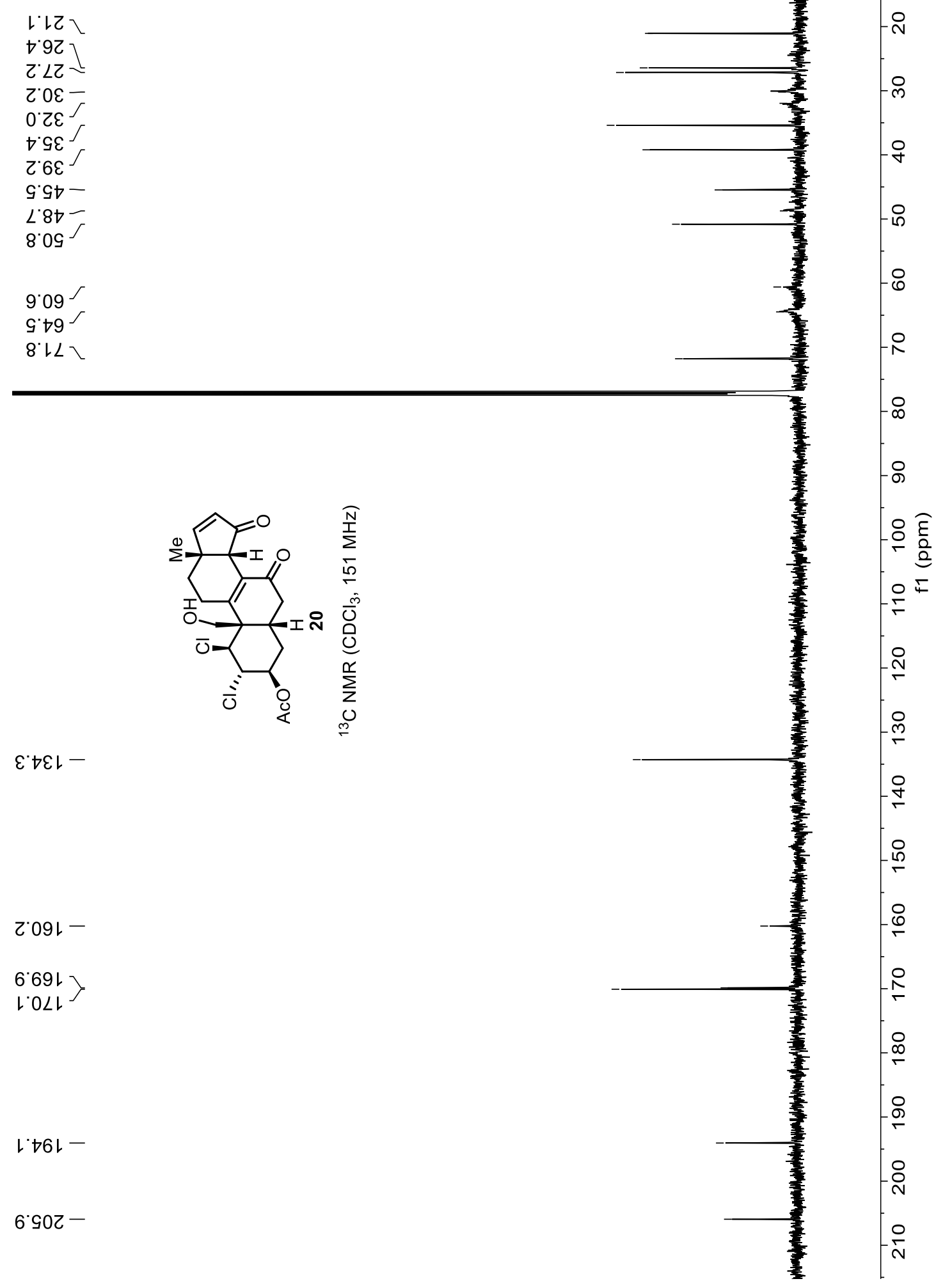




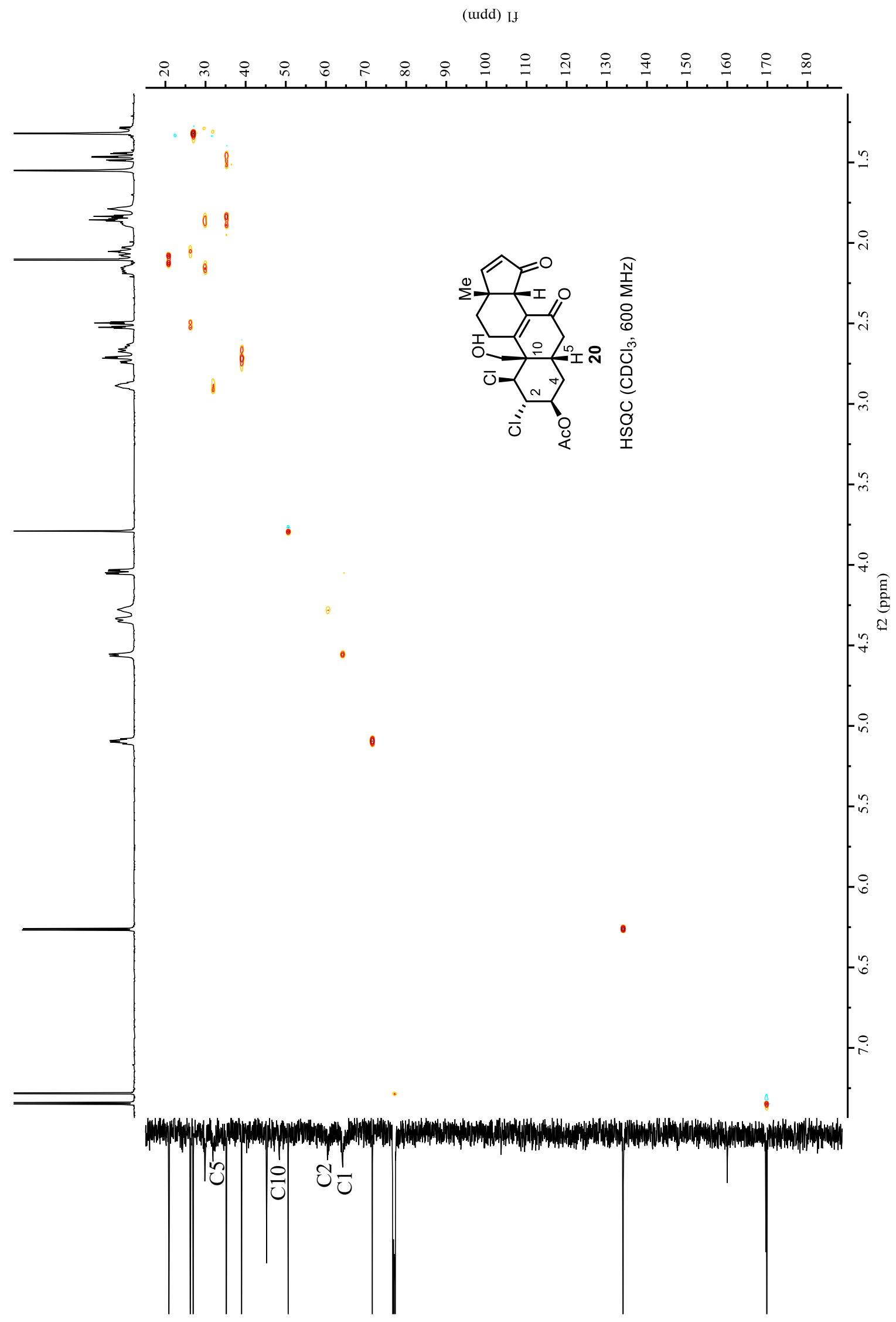


(udd) If

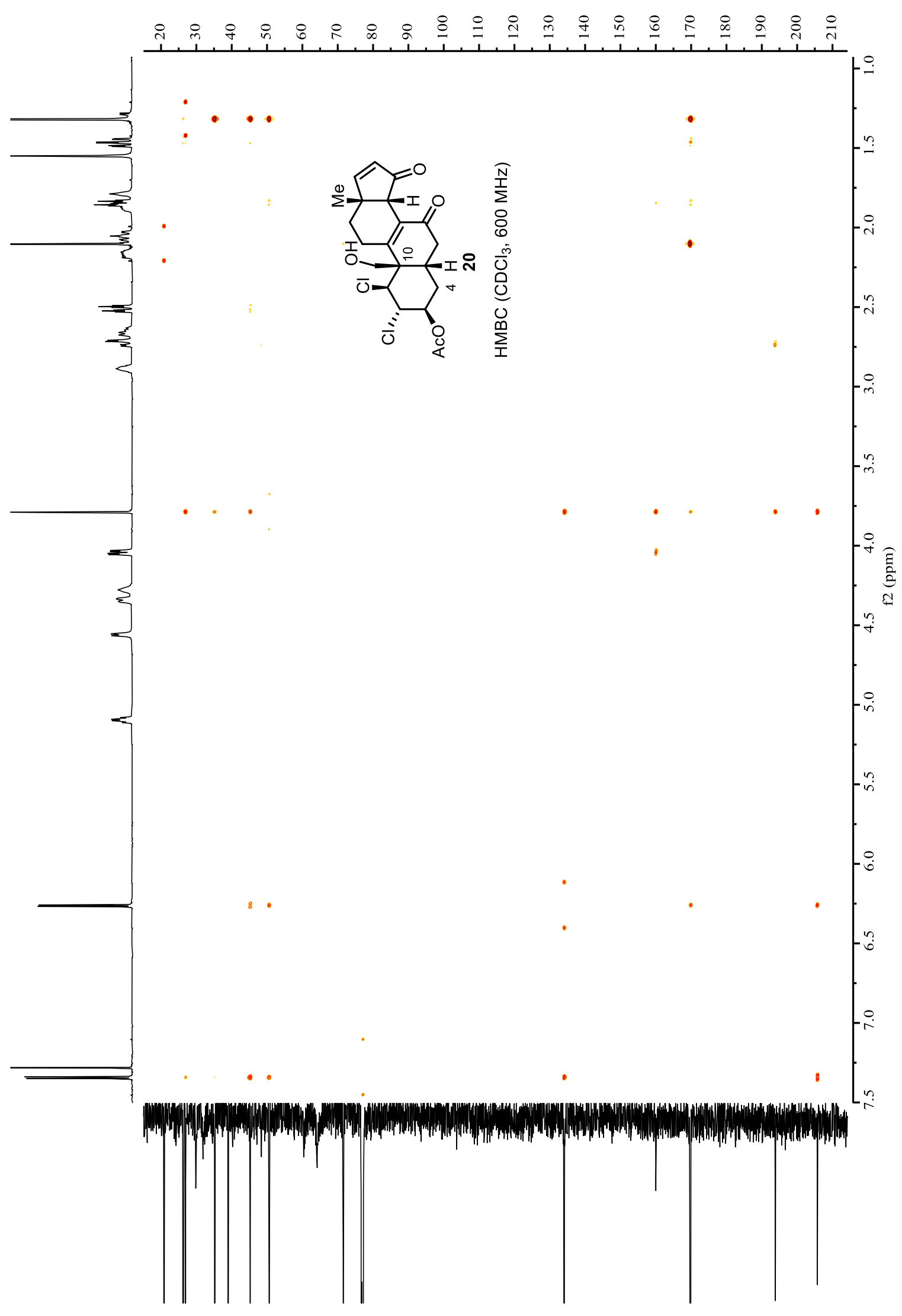




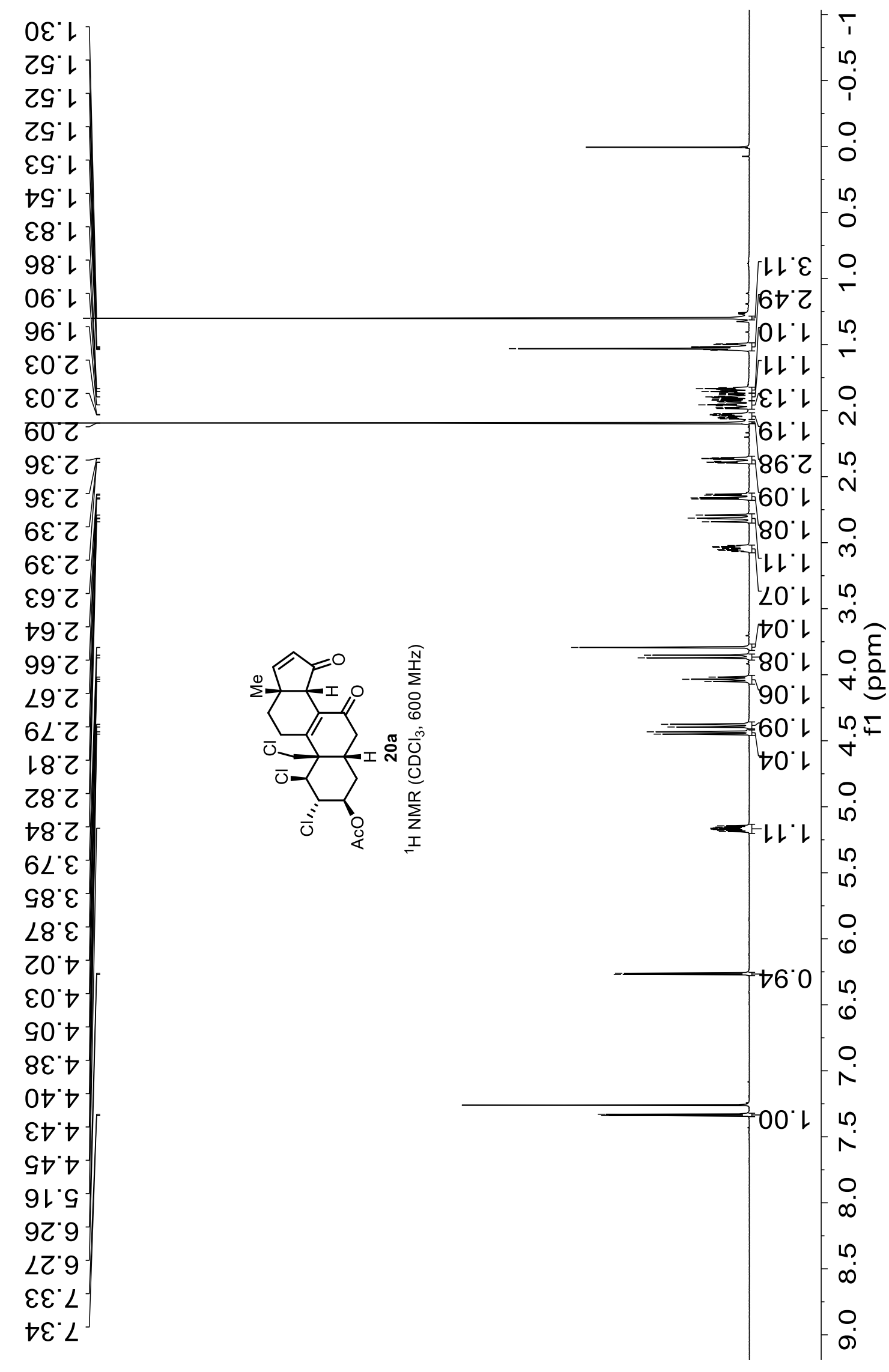



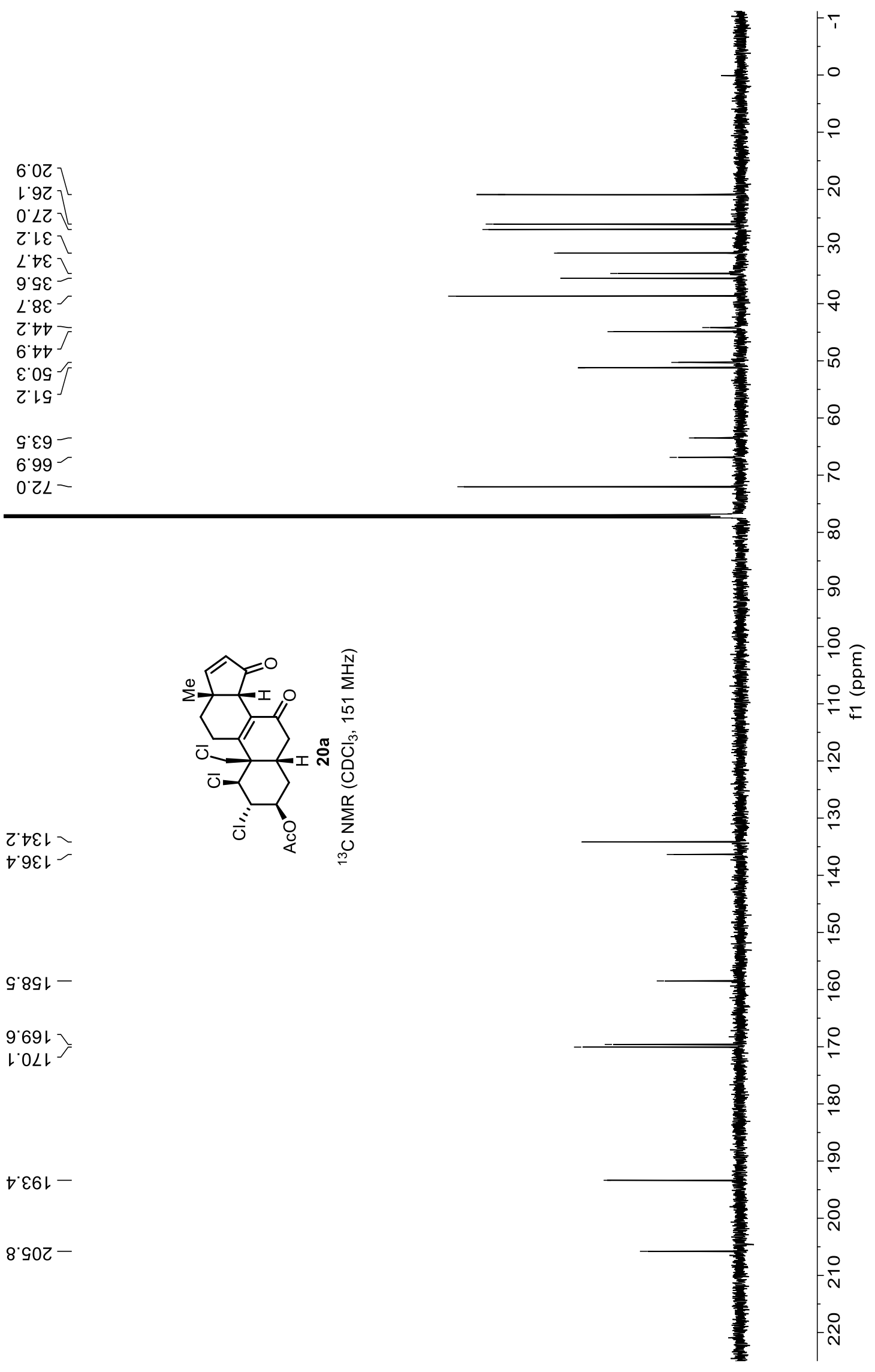
(udd) If
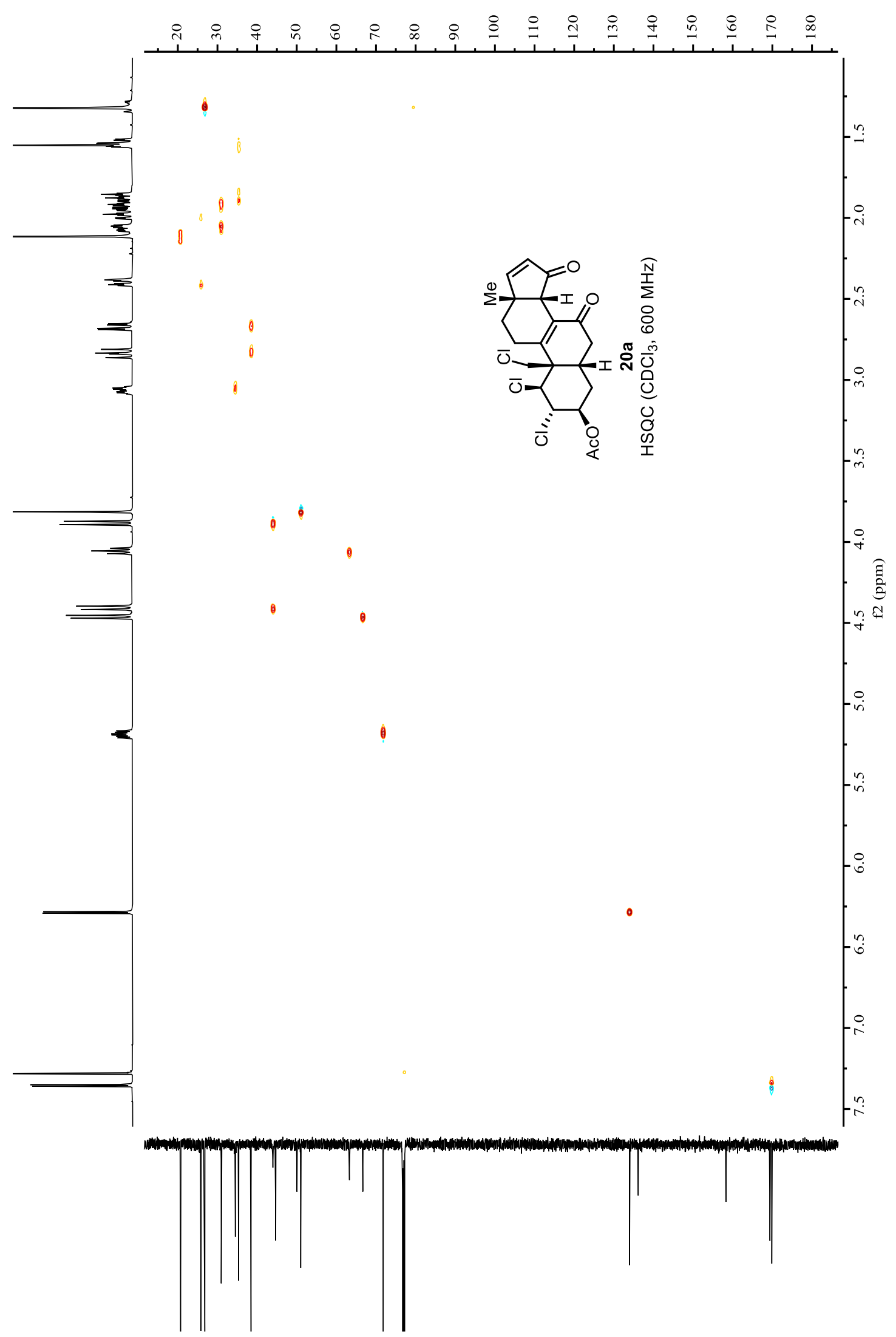


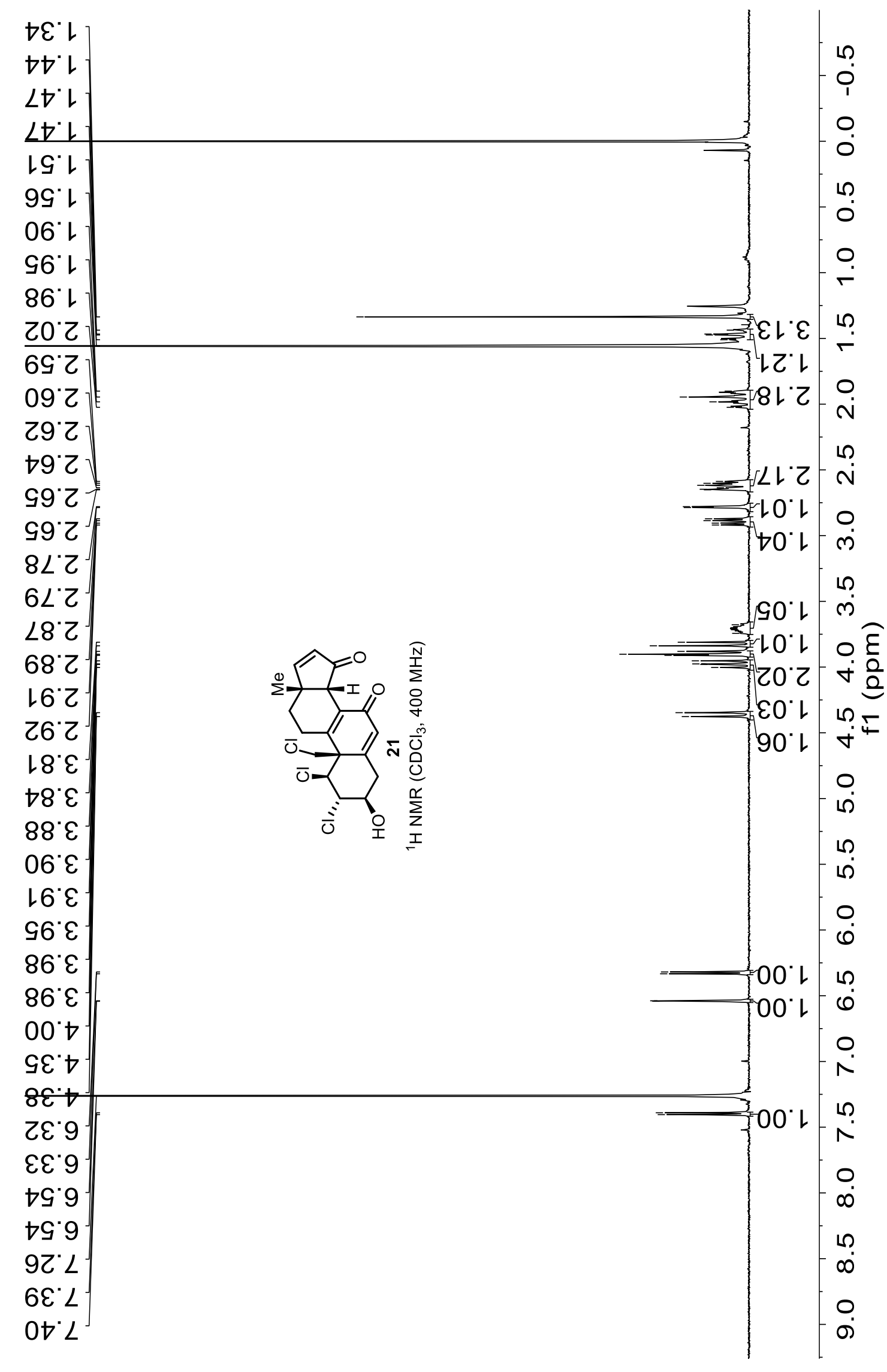




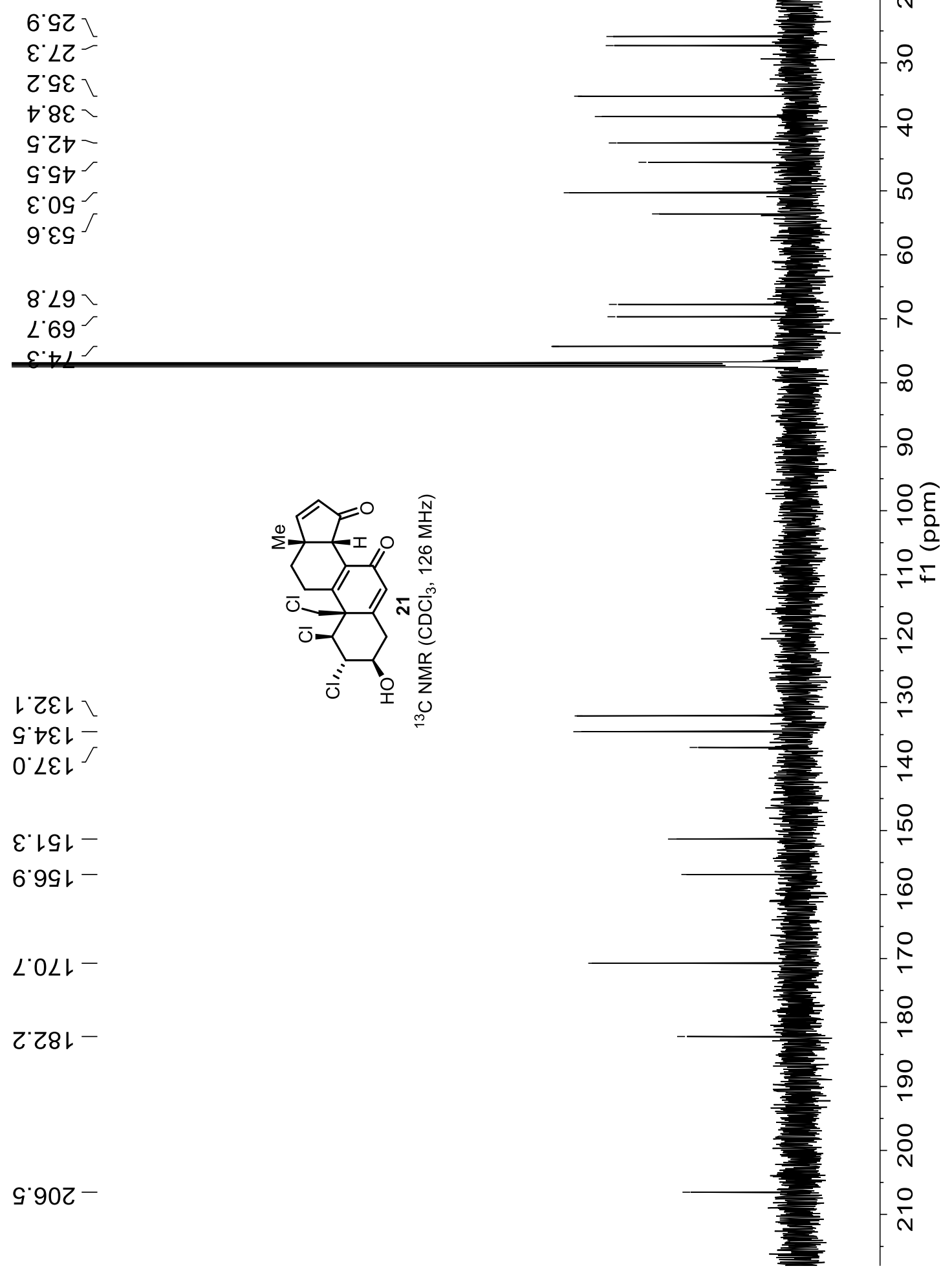


(uidd) If

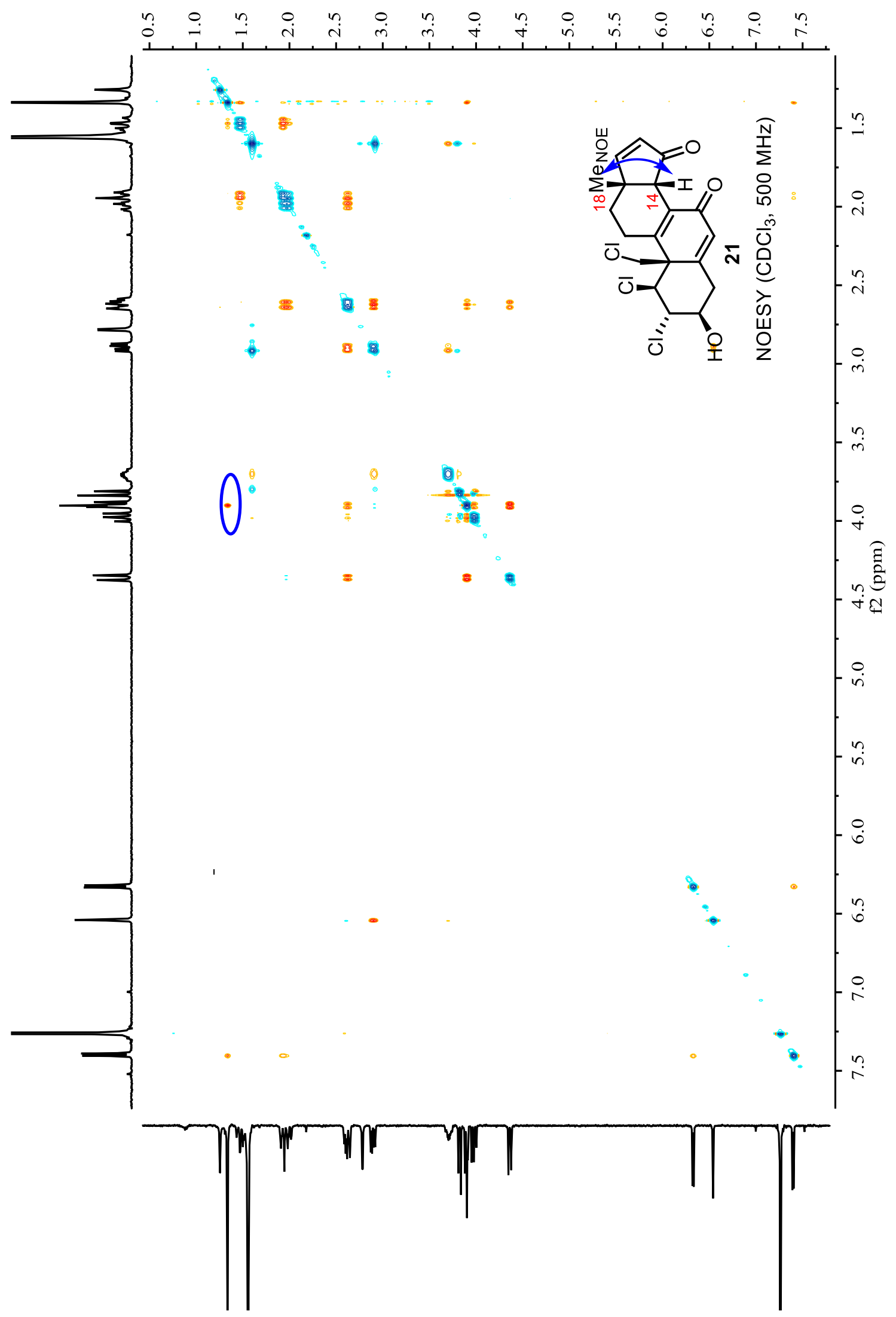




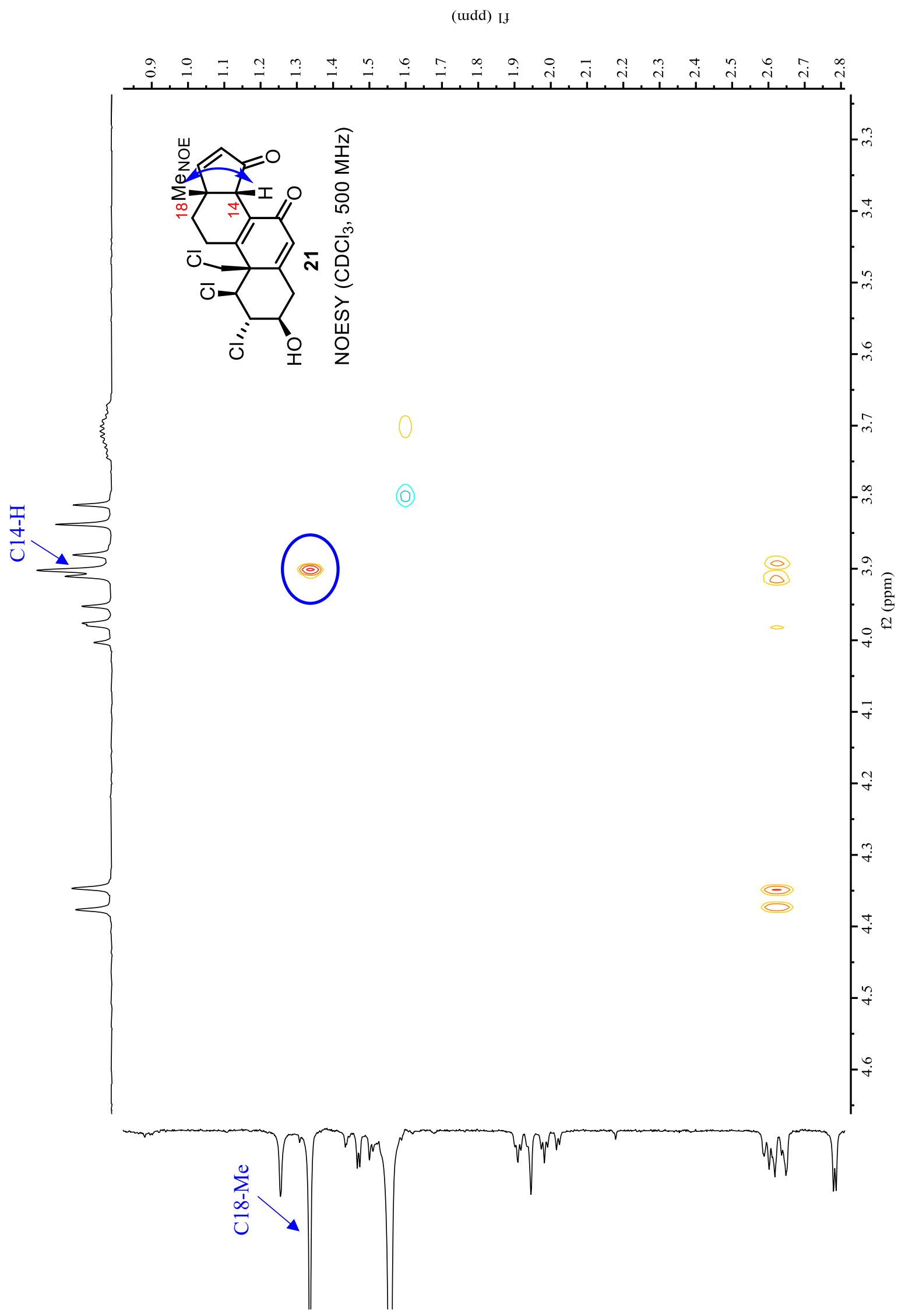




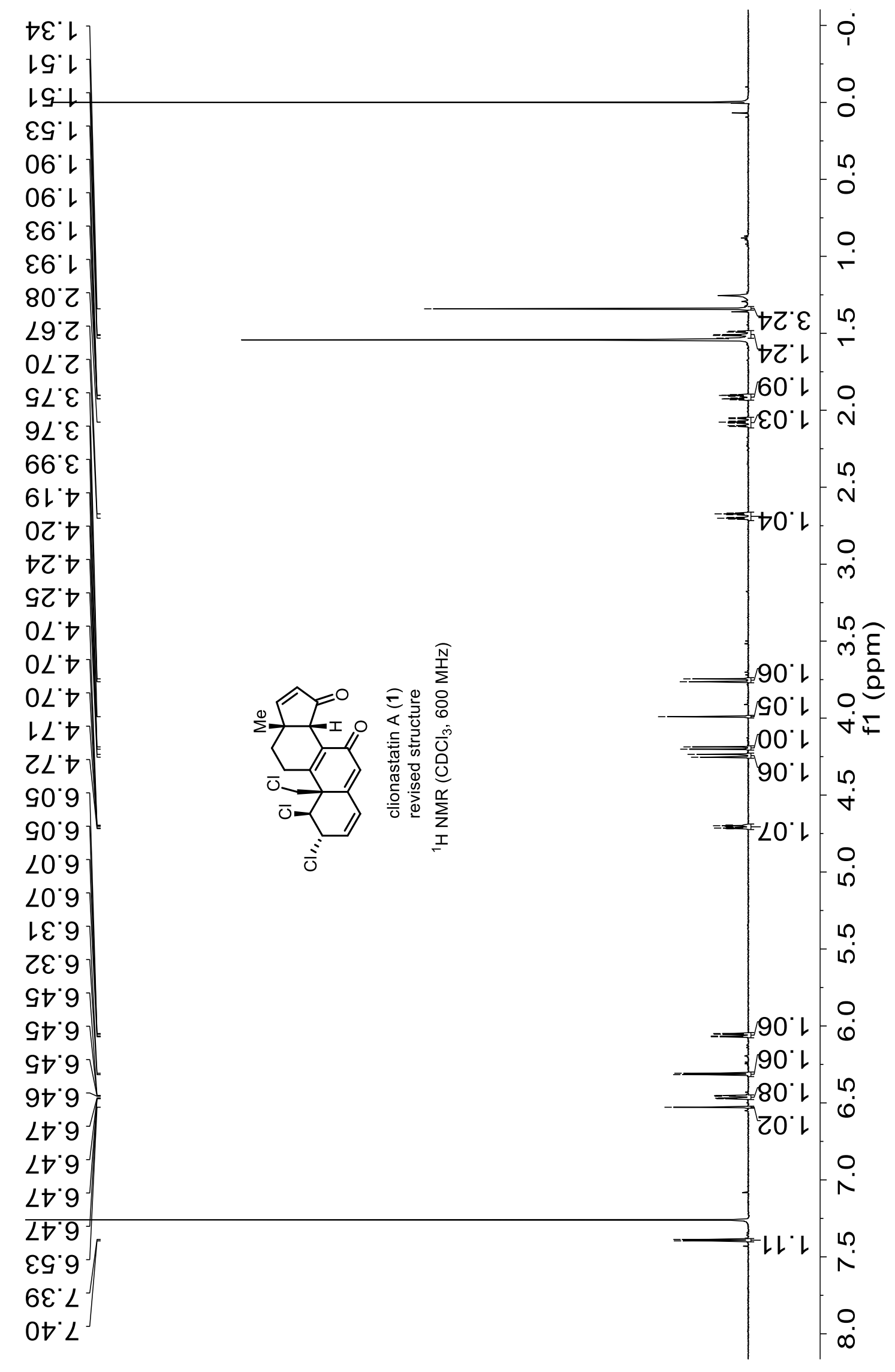




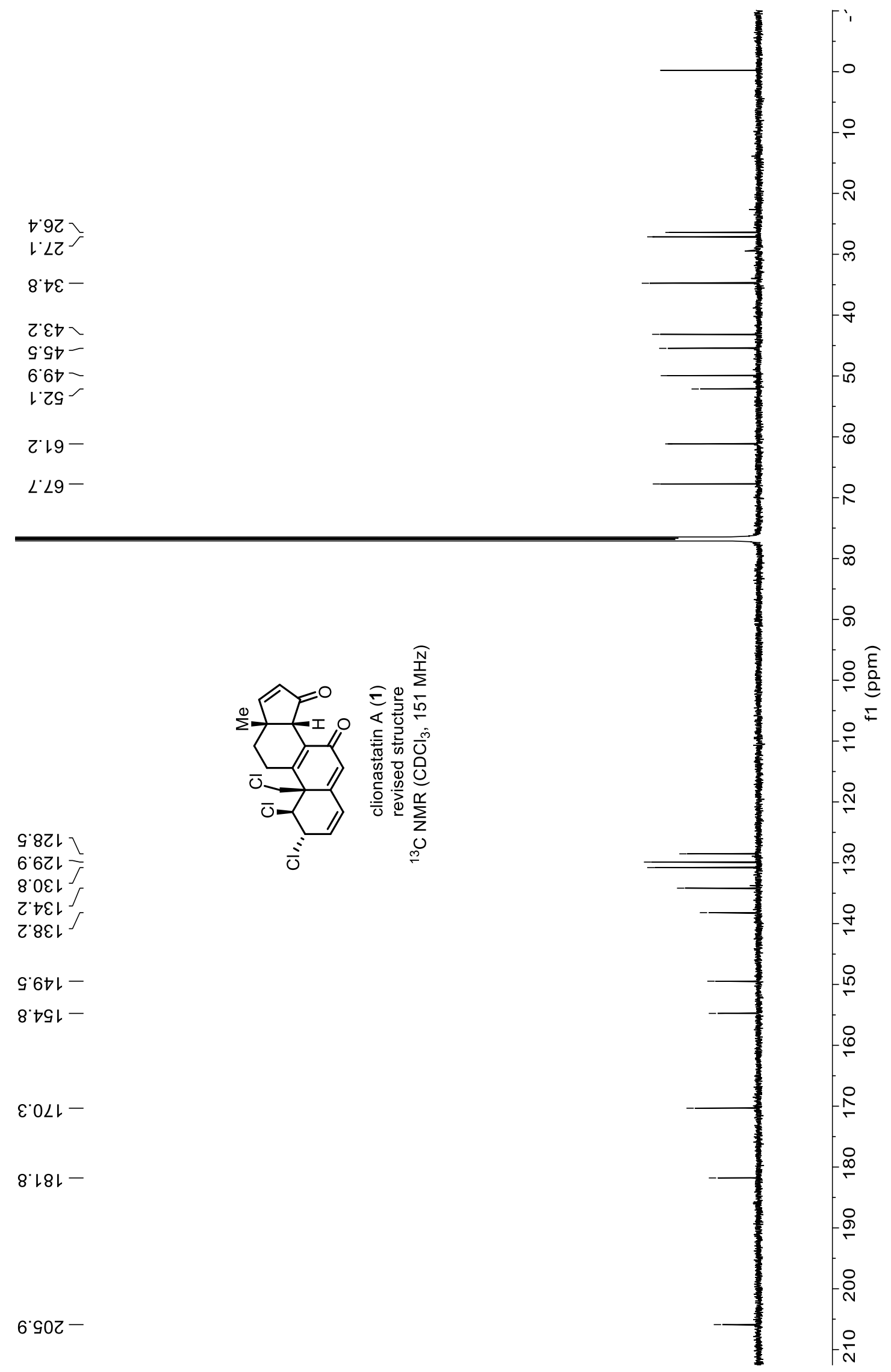




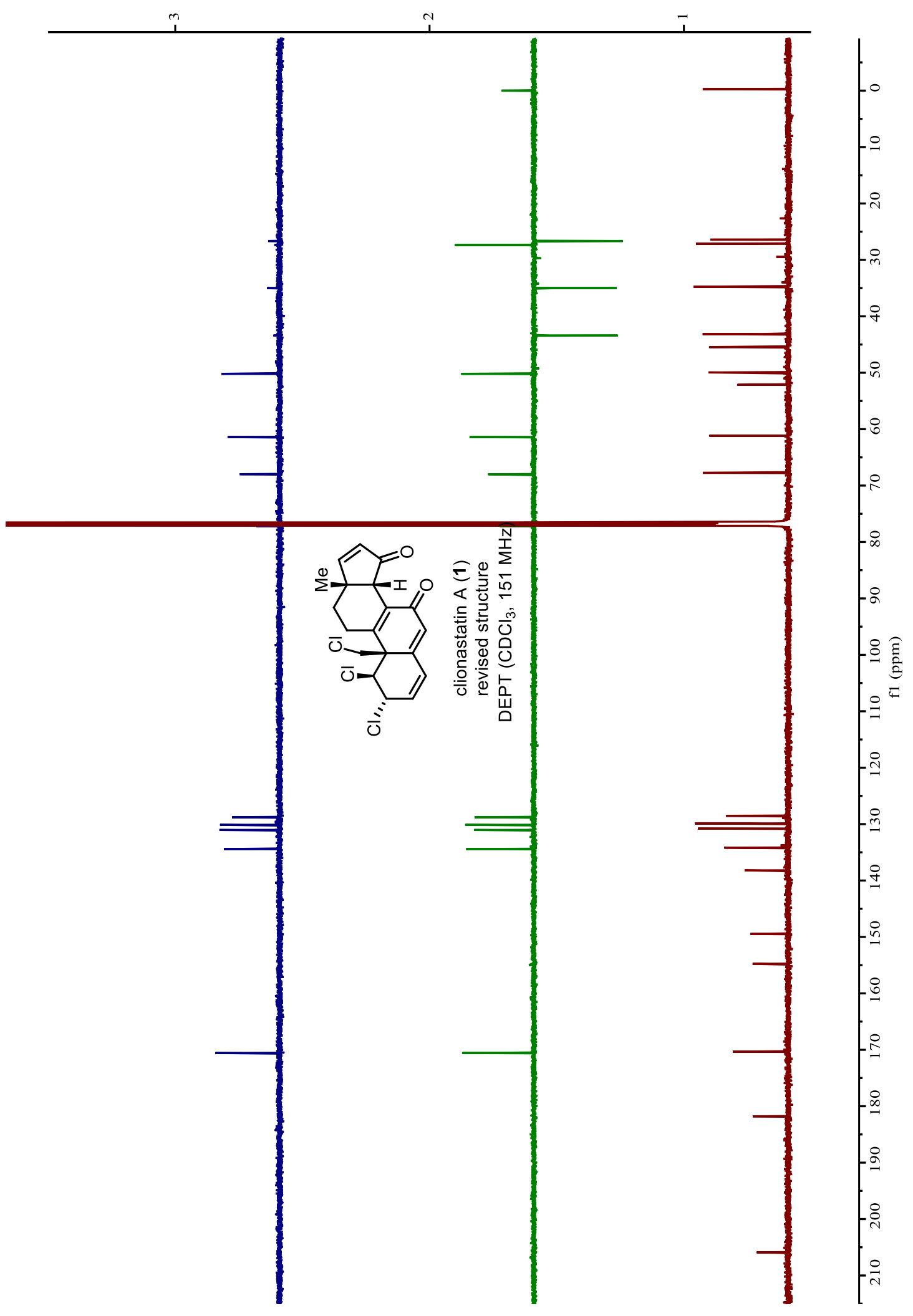




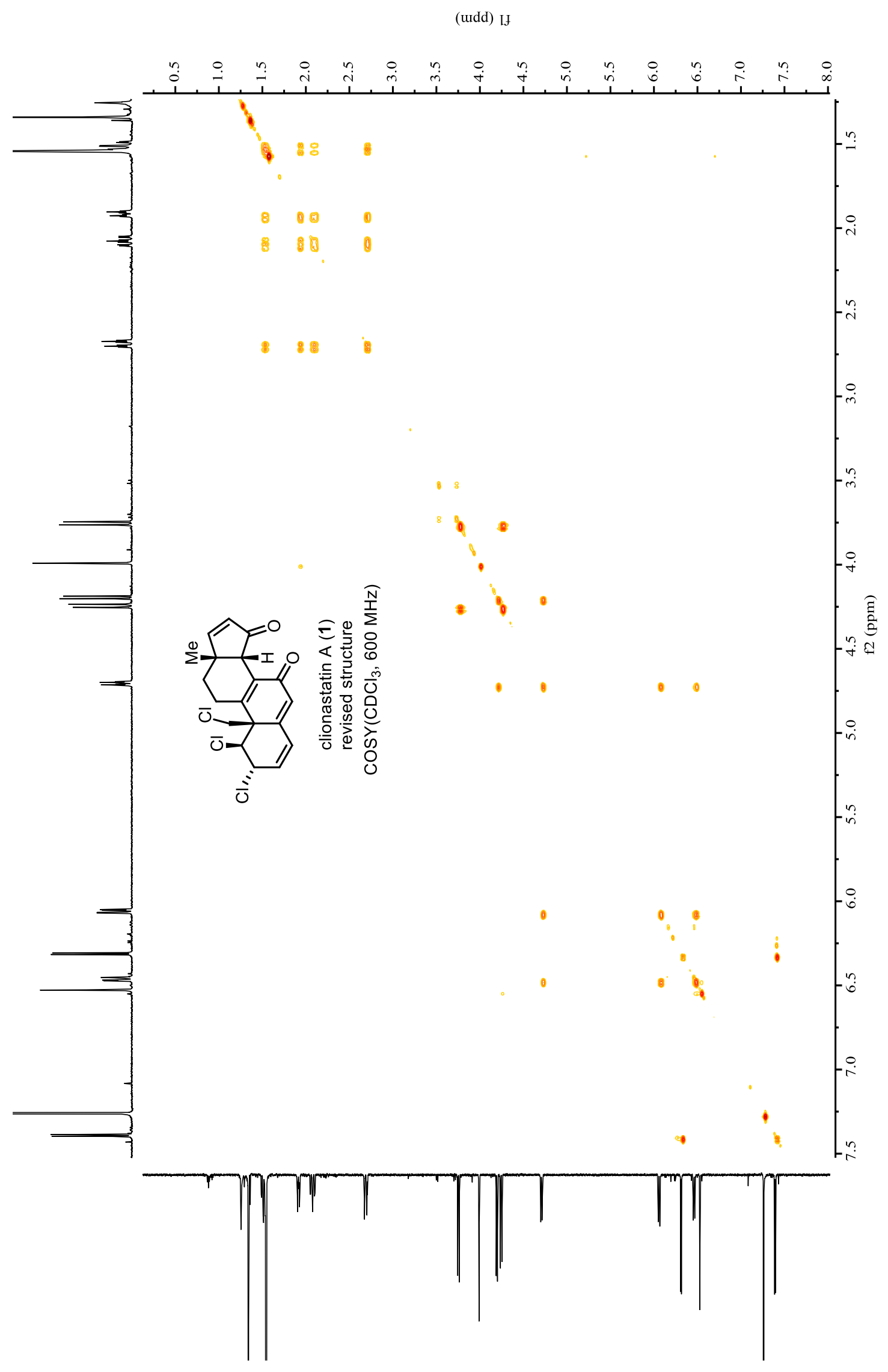




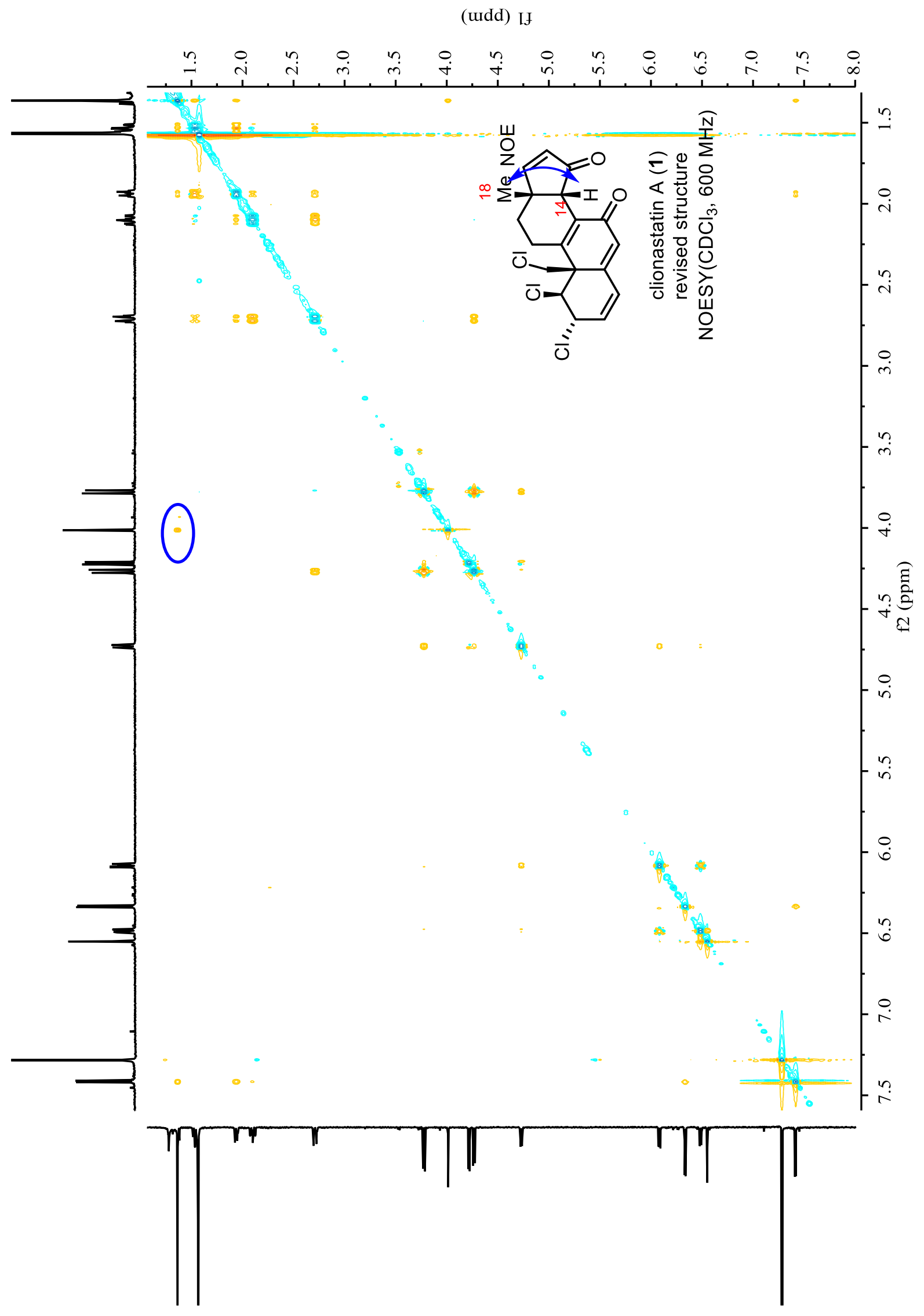




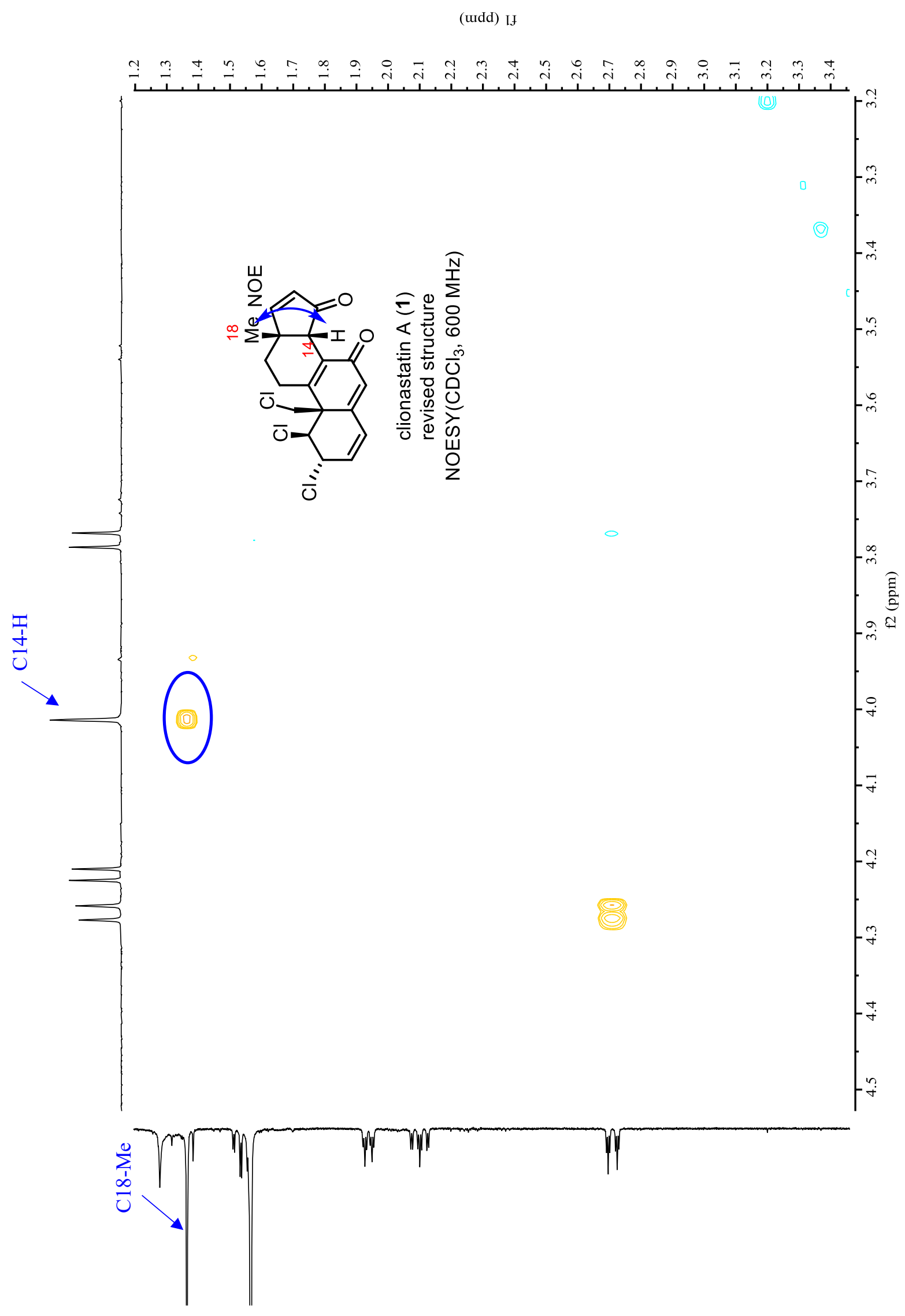


(udd) If

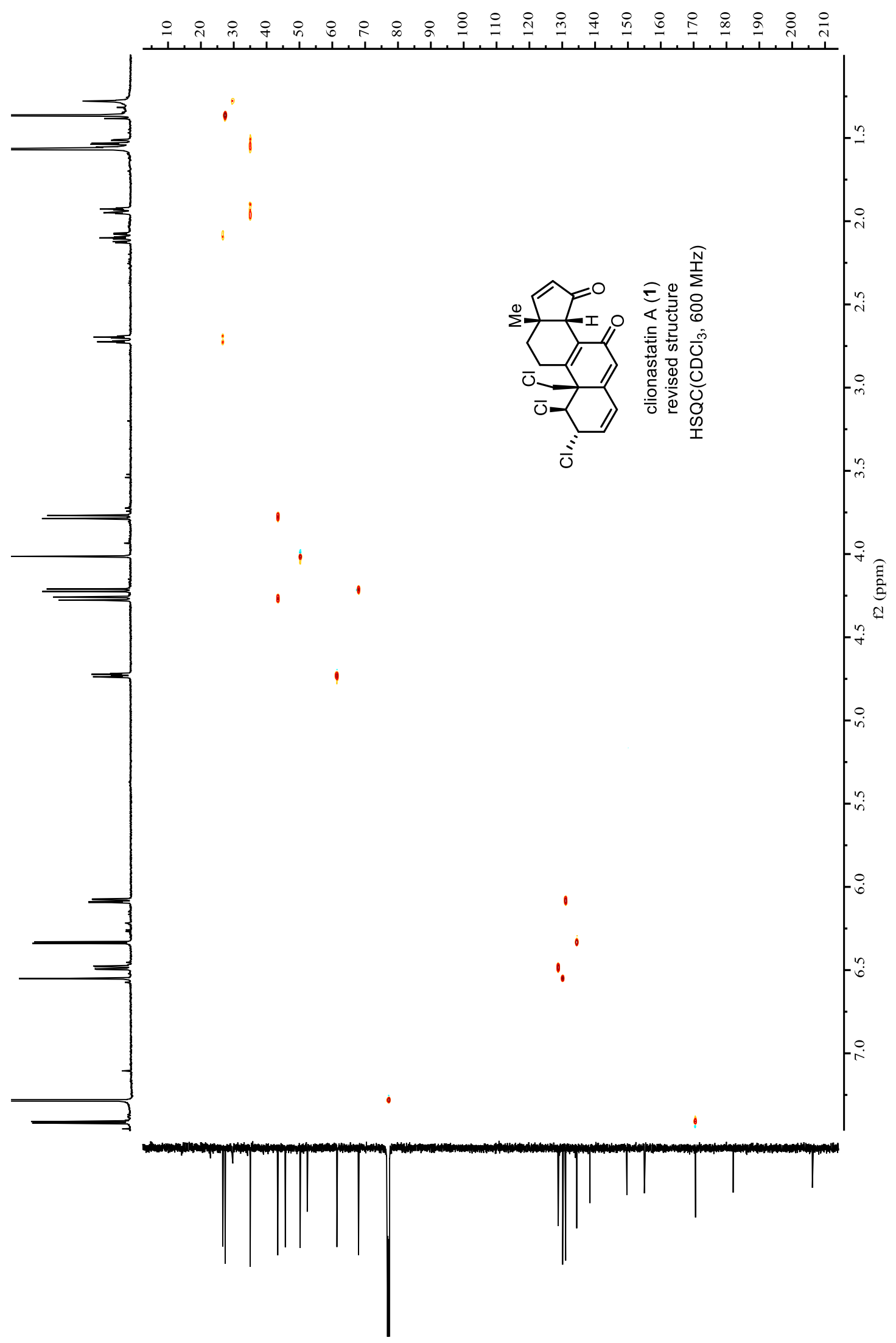


(udd) If

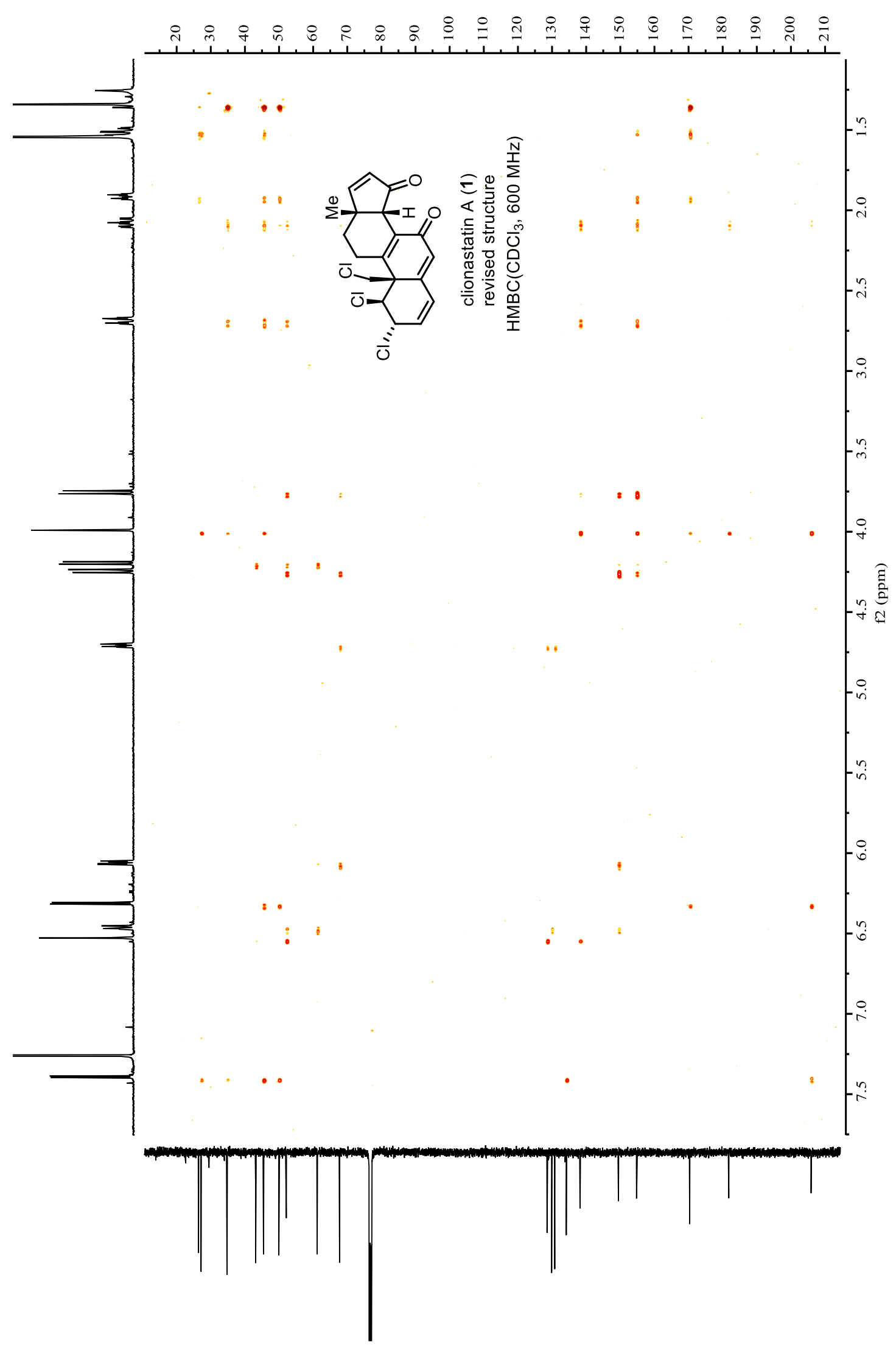




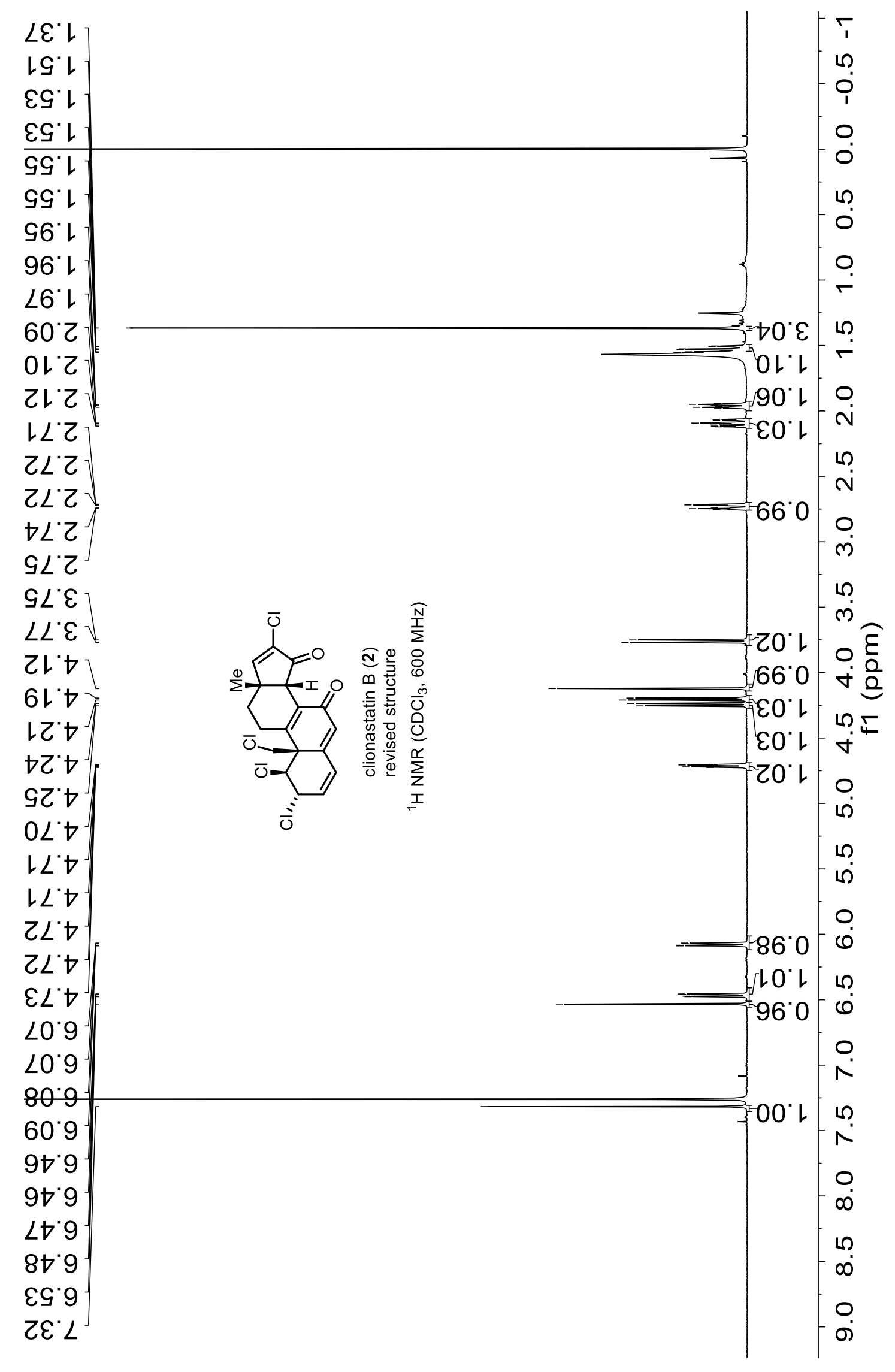




$$
\begin{aligned}
& \begin{array}{l}
\text { S. } 9 Z \bar{J} \\
\text { † } L Z
\end{array} \\
& 0 \mathrm{Q} \varepsilon- \\
& \left.\begin{array}{l}
l \cdot \varepsilon t \\
\tau \cdot \varepsilon t \\
l \cdot \sigma t
\end{array}\right\} \\
& \text { l.6t- } \\
& \varepsilon \mathcal{Q ⿻}^{-} \\
& \text {1.19- } \\
& \text { L } \angle 9-
\end{aligned}
$$
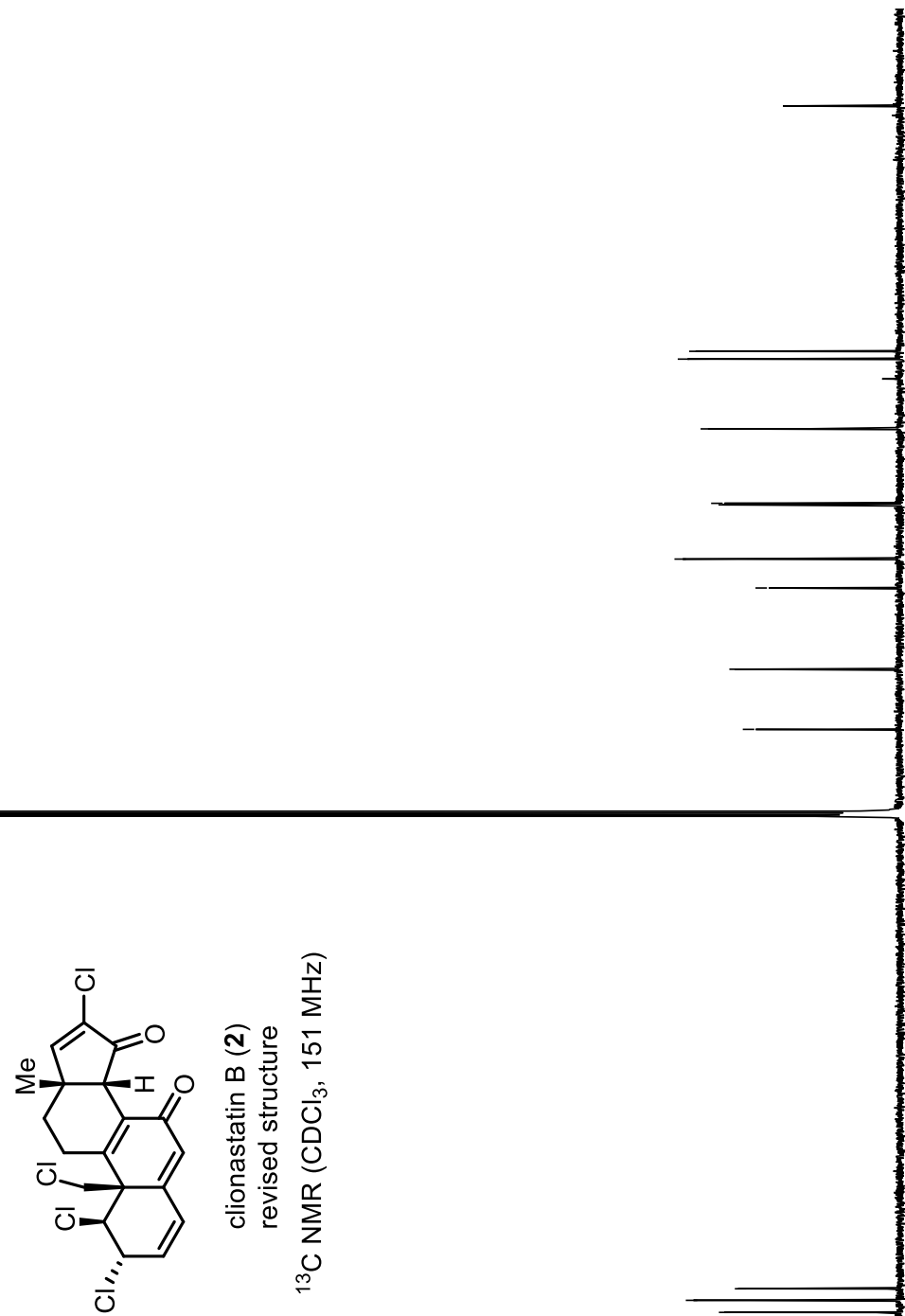

t.181-

$\varepsilon^{\prime} \angle 6 L-$

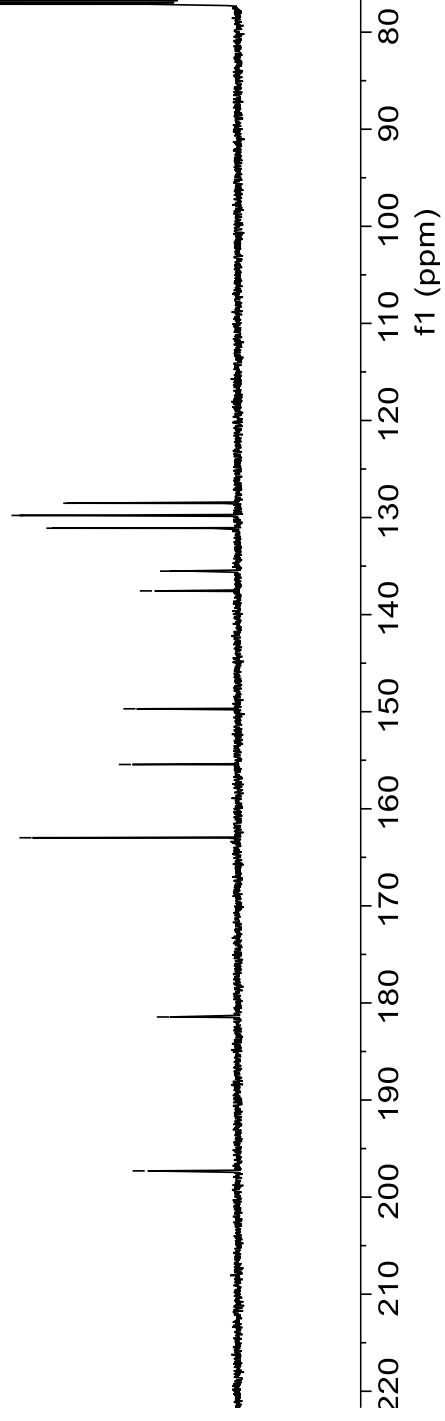




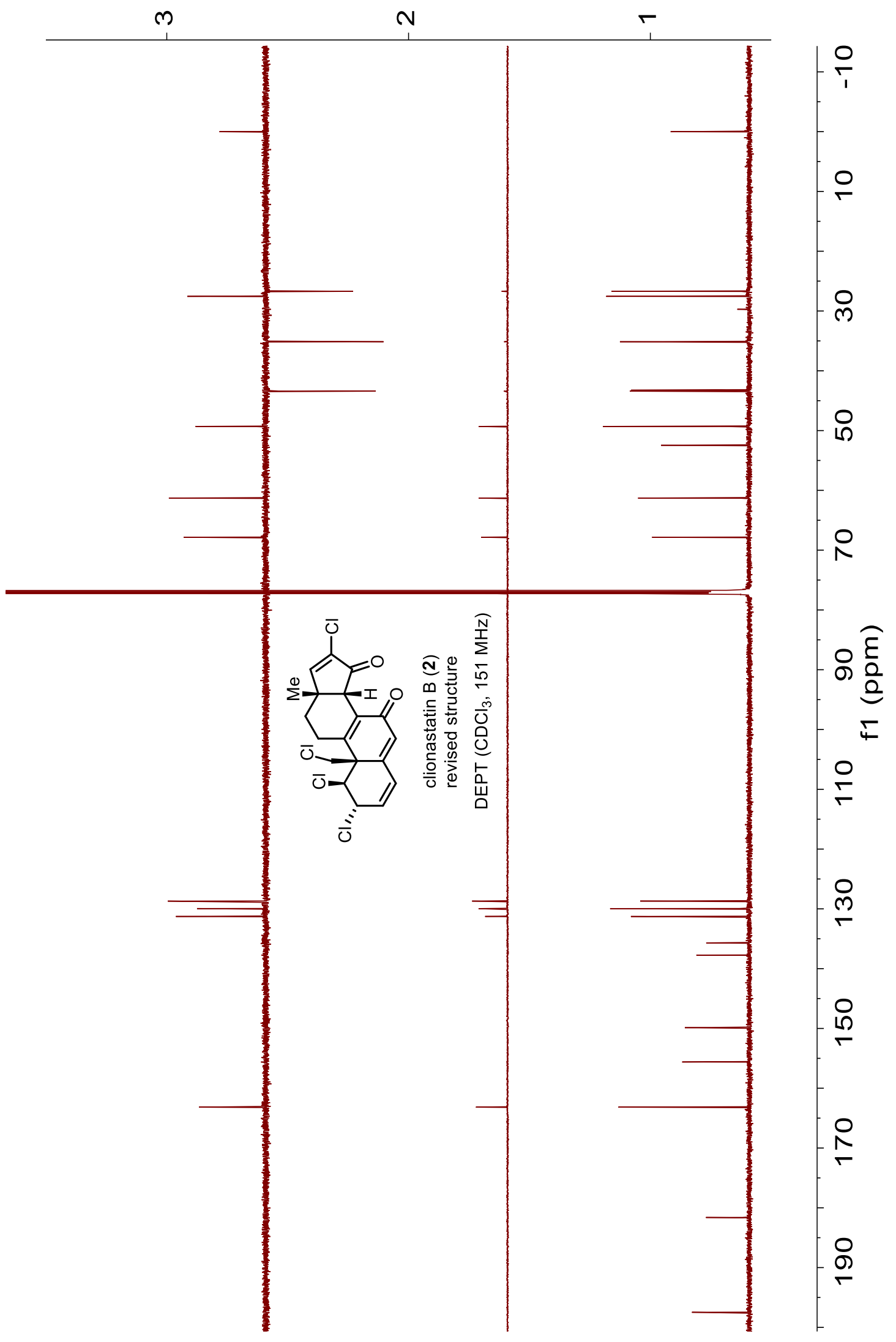




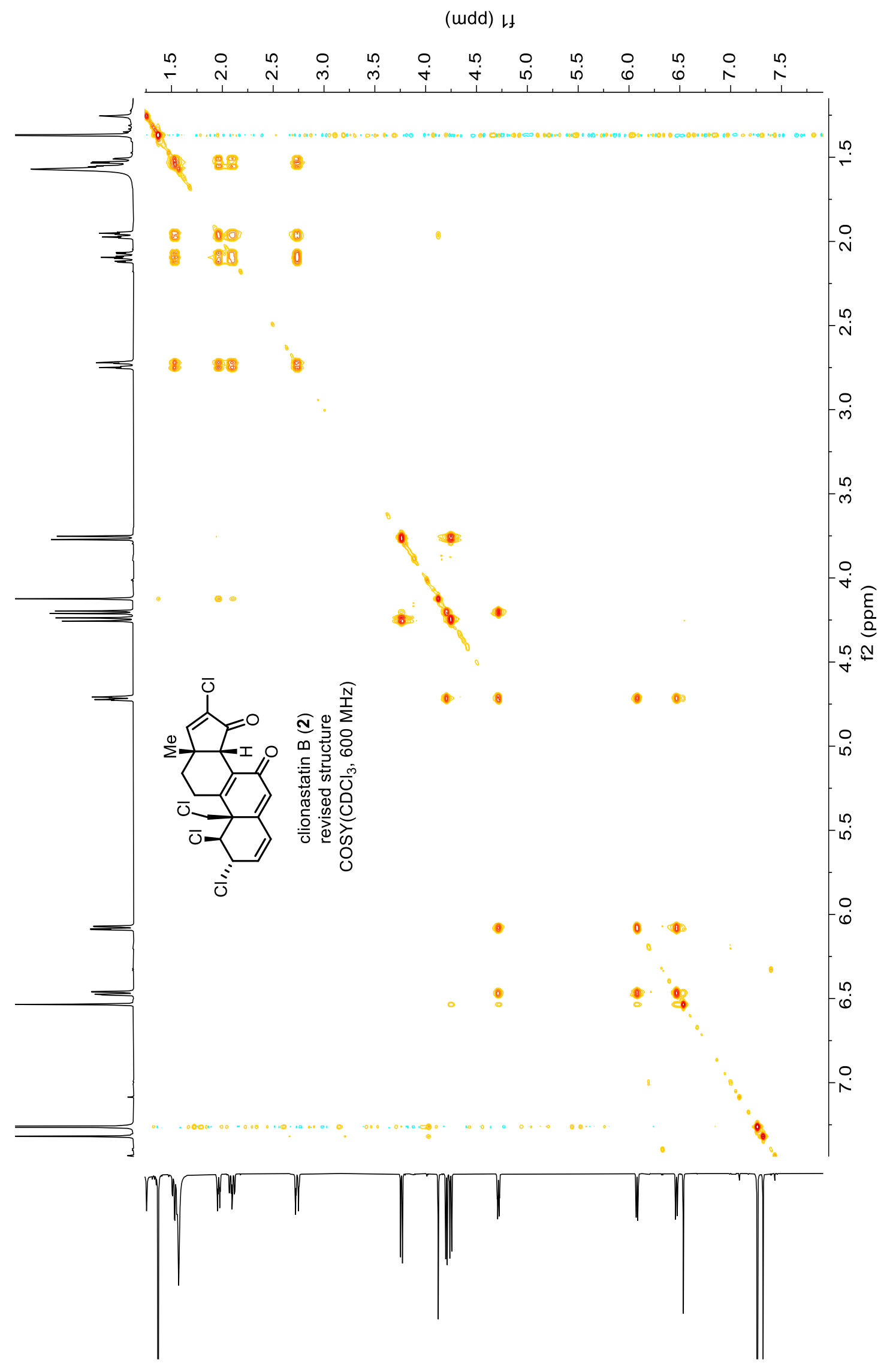




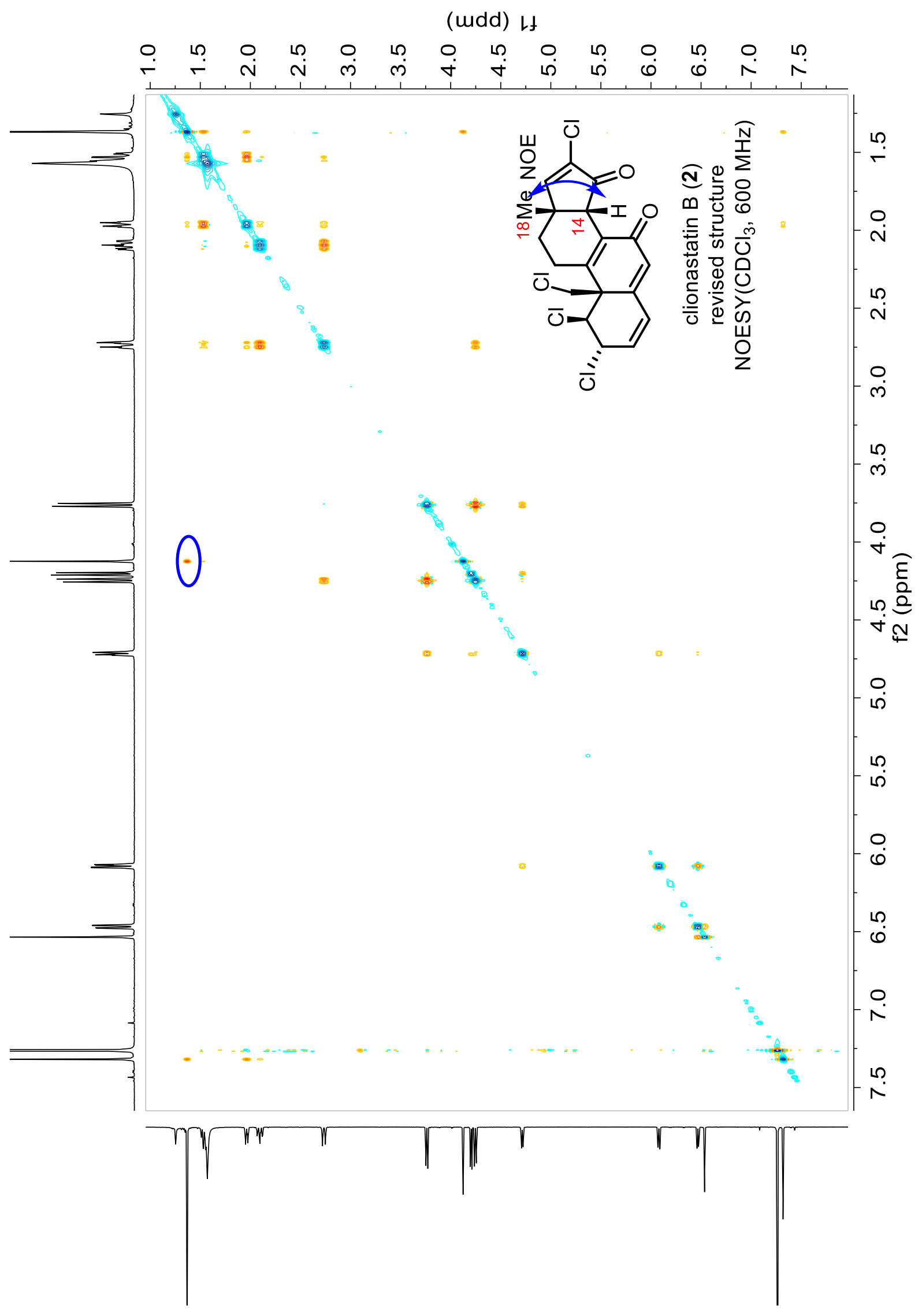




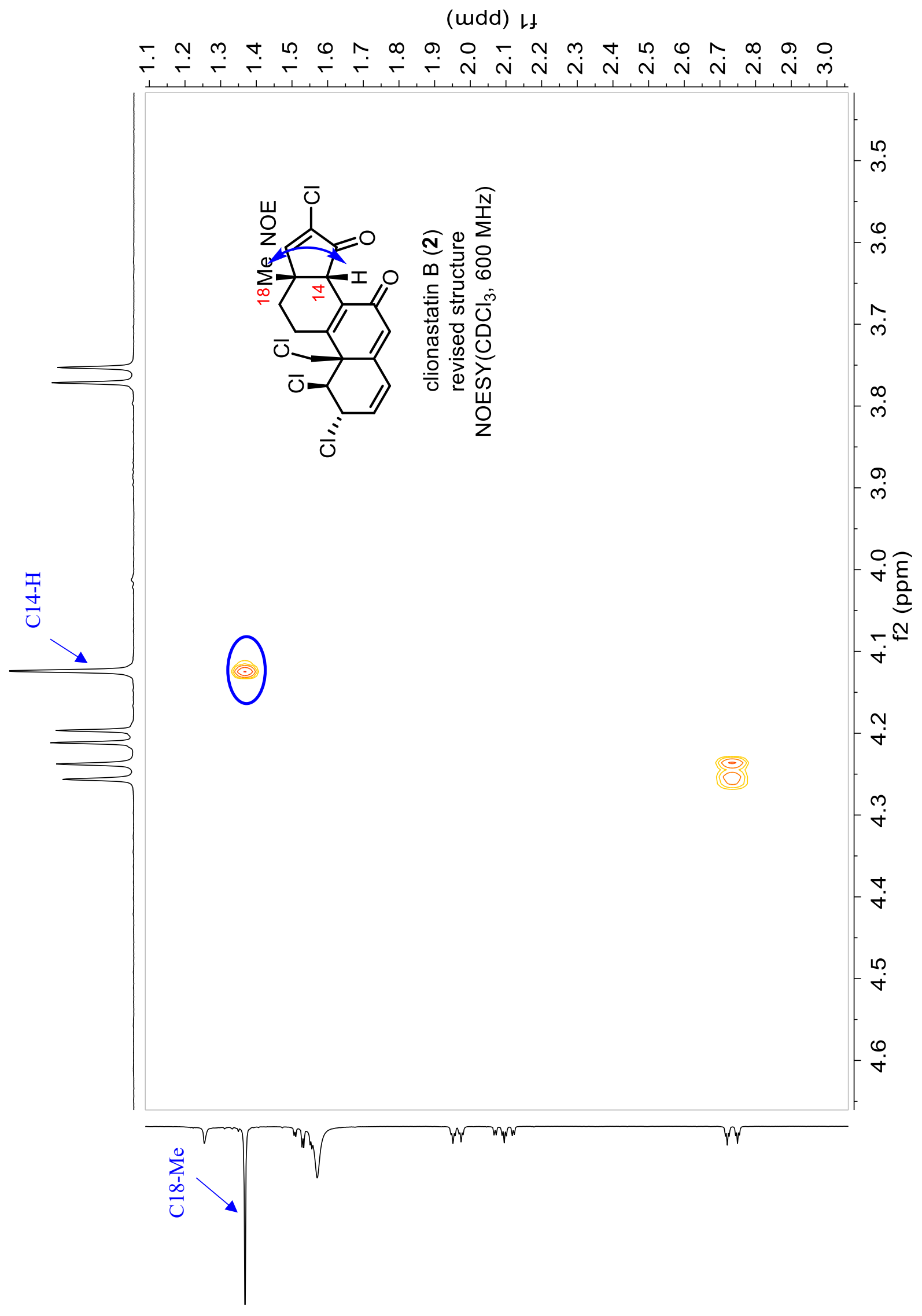




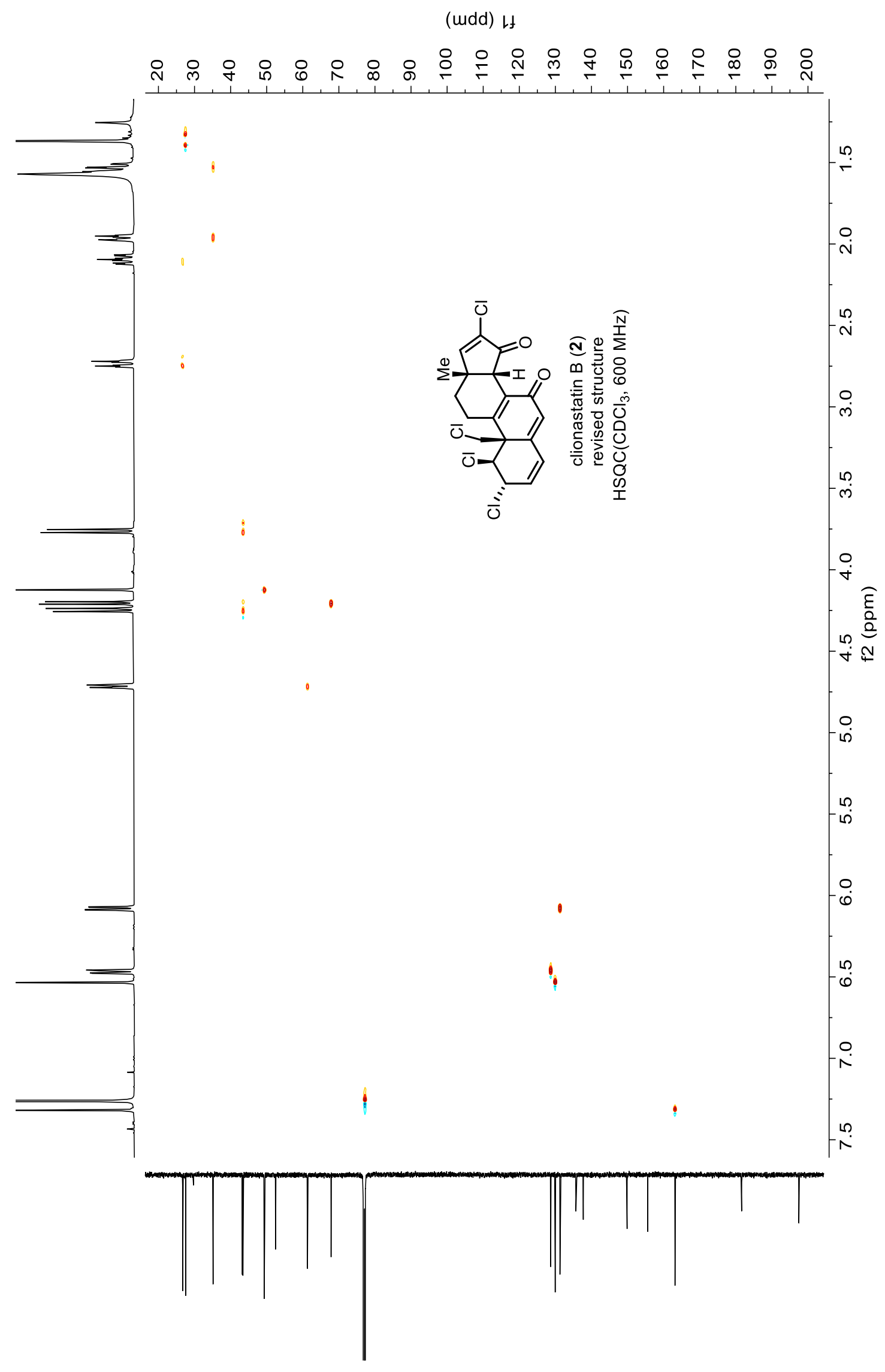




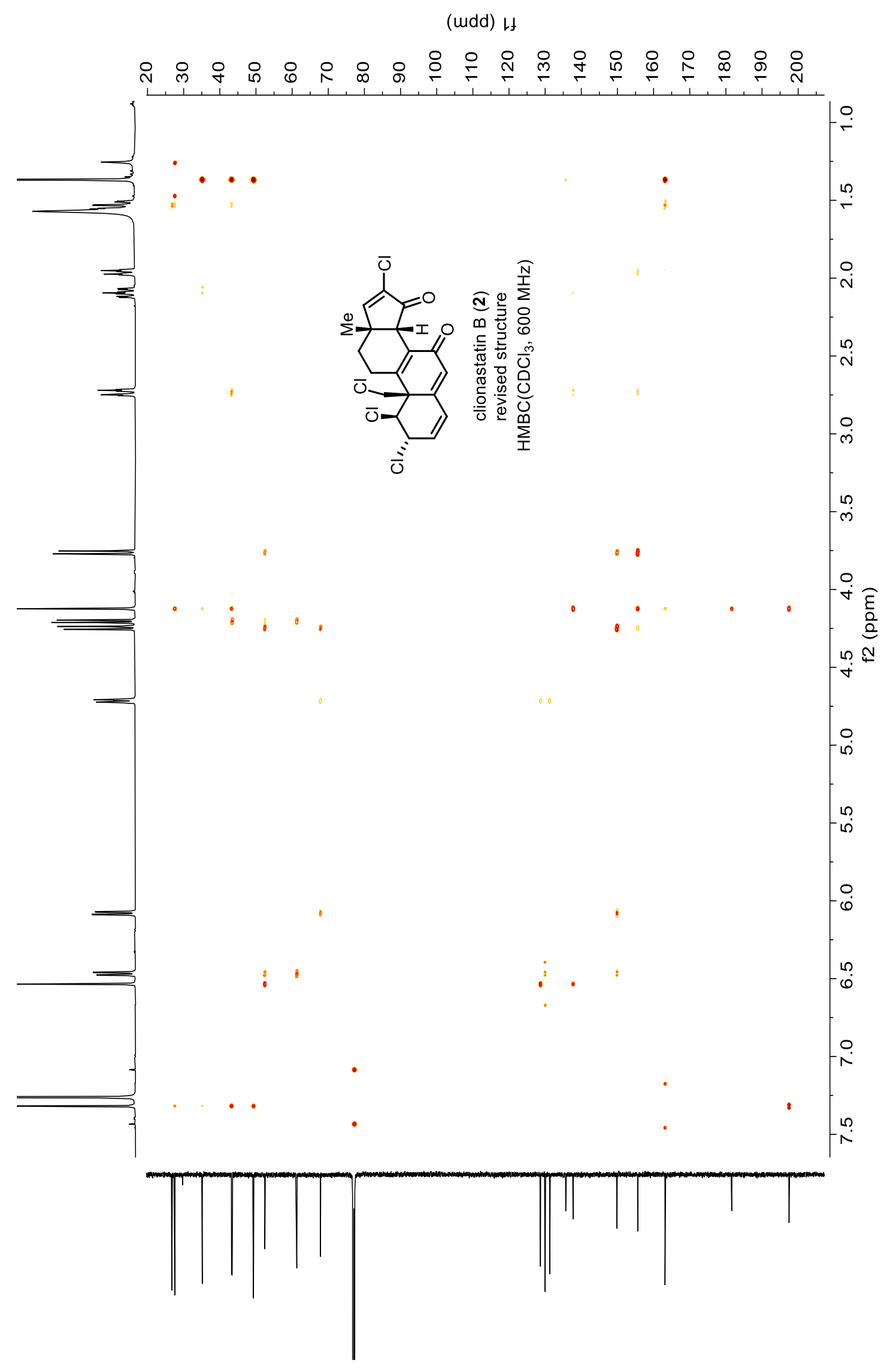




\section{References}

(1) Schlama, T.; Gabriel, K.; Gouverneur, V.; Mioskowski, C. Tetraethylammonium Trichloride: A Versatile Reagent for Chlorinations and Oxidations. Angew. Chem. Int. Ed. 1997, 36, 2342-2344.

(2) Hu, D. X.; Shibuya, G. M.; Burns, N. Z. Catalytic Enantioselective Dibromination of Allylic Alcohols. J. Am. Chem. Soc. 2013, 135, 12960-12963.

(3) Bucher, C.; Deans, R. M.; Burns, N. Z. Highly Selective Synthesis of Halomon, Plocamenone, and Isoplocamenone. J. Am. Chem. Soc. 2015, 137, 12784-12787.

(4) Hu, D. X.; Seidl, F. J.; Bucher, C.; Burns, N. Z. Catalytic Chemo-, Regio-, and Enantioselective Bromochlorination of Allylic Alcohols. J. Am. Chem. Soc. 2015, 137, 3795-3798.

(5) Landry, M. L.; Hu, D. X.; McKenna, G. M.; Burns, N. Z. Catalytic Enantioselective Dihalogenation and the Selective Synthesis of (-)-Deschloromytilipin A and (-)-Danicalipin A. J. Am. Chem. Soc. 2016, 138, 5150-5158.

(6) Seidl, F. J.; Burns, N. Z. Selective bromochlorination of a homoallylic alcohol for the total synthesis of (-)-anverene. Beilstein J. Org. Chem. 2016, 12, 1361-1365.

(7) Xu, S.; Holst, H. M.; McGuire, S. B.; Race, N. J. Reagent Control Enables Selective and Regiodivergent Opening of Unsymmetrical Phenonium Ions. J. Am. Chem. Soc. 2020, 142, 8090-8096.

(8) O'Connor, M. J.; Sun, C.; Guan, X.; Sabbasani, V. R.; Lee, D. Sequential 1,4-/1,2-Addition of Lithium(trimethylsilyl)diazomethane onto Cyclic Enones to Induce $\mathrm{C}-\mathrm{C}$ Fragmentation and $\mathrm{N}-\mathrm{Li}$ Insertion. Angew. Chem. Int. Ed. 2016, 55, 2222-2225. 